MULTIPLE-BEAM SPECTROSCOFY

\title{
Contents
}

Abstract

I. Introduetion

A. Optical Diagnostic Techniques

B. Plasma Spectroscopy

C. The Use of Coherent Light: Interferometry and

D. Information in the Emitted Lighi

II. The Use of Phase Information

A. A Two-Beam Spectrometer

1. Ilght from an Incoherent Source: The Sum of Many Interference Patterns

2. Some Comments on There Results

3. A Reformulation of the Two-Beam Problem

B. Multiple-Beam Systems

1. The Ise of Polarization

2. Multiple-Beam Systems in the Huygens

Approxiration

3. Three Particular Opt1cal Systems

$\checkmark$

c. Higher order Correlations

III. SIgnel, Nolse, and Light Intensity

A. Quantum Optics and Inteneity Correiations 88

B. Claselcal Notse

C. An Estimate of Photon Statistics 
IV. Experimental Results

A. Tests of the Opt1cal System 103

1. The Calcite Rhomb 103

2. A Two-Beam Spectroscopic System 109

3. Some Multiple-Beam Systems 117

4. A System with Several Independently Collimeted Pairs of Beams 126

B. Observetions of a Plasma 133

v. H1gh-Frequency Phenomena 149

A. The Effect of a Moving Source and the Use of a Time-Varying Optical System $\quad 149$

B. Correlations between light of Different

Frequencies $\quad 158$

$\begin{array}{ll}\text { VI. Conclusions and Suggestions } & 167\end{array}$

A. A Sumary of Results $\quad 167$

B. Extension of flais Work 173

c. F'inal Commeris 178

Acknowledgments $\quad 183$

Appendices

A. Holography, Srsctrosccy, and Scattering 184

B. Some Mathematical Details 190

1. An Integration Needed in Sect. I.C. 190

2. An Integration Needed in Sect. II.A.3 194

c. The Design of a Multiple-Besw Spectroscopic $\begin{array}{ll}\text { Apparatus } & 197\end{array}$

1. Previous Conclusions 197

2. A Modifled Two-beam System 299 
3. The Use of Birefringence 204

4. Multiple-Beam Systems 213

5. Spectral Width, Beam Divergejce, and the Quality of the Optical Components 226

D. Studies of the Plasma with Iangmuir Protes and with Conventionel Spectroscopy 232

E. Some Notes on the Definfticis of spectra 237

1. Direction Dependent Spectra 237

2. A Property of the Analytic Sfgnel 249

3. T1me-Dependent Spectra 251

F. Notation 256

Footnotes and References 265 


\section{MULTIPLE-BEAM SPECTROSCOPY}

Peter s, Rostler

ABSTRACT

Th1s report describes a new spectroscopic technique which provides spatially localized information about fine sca.le fluctuations in the density of light sources within a self-luminous plesma. In conventiongl spectroscoplc methods, only the frequency spectmin of the light is measured. Yet light is characterized by phase as well as frequency. If a source is observed from several directions (through. several beams), one can measure the correlations in phese between light emitted in different directions. With en incoherent source, two-beam correlations c'sl only be due to common sources, 1.e., to light emitted from within that small region which is observed through both beams. Thus the result of a correlation messurement is not an average along a line of sight; It is ab local meesurement.

It is shown that the light accepted by a two-beam system can be described in terms of spatial Fourler transforms of the fleld. The mutual coherence between light of wave number $\left|\underline{k}_{A}\right|=\left|\underline{k}_{B}\right|$ emitted in directions $\hat{\mathrm{k}}_{A}$ and $\hat{\mathrm{k}}_{B}$ is then shown to be proportional to the $k_{\Delta}=k_{B}-k_{A}$ Fourler component of the light source distribution. This result is similar to what is found in an anelysis of laser-light scattering. Thus the type of inforration given by a scattering experiment can glso be obtained from spectroscopic measurements upon the light emitted by the plasma itself. Multiplebeam spectroscopy and laser-light scattering differ, however, 
both in applications and in besic princlples, as is explained in a detailed comparison of the two methods.

A two-beam spectrometer is only the simplest of many possible Instruments of this type. For applications one needs to employ a more efficient system--one which presents a much larger solid angle of acceptance. It is shown how this can be convenlently done with polarizing optics, using birefringent optical comporients to manipulate two sets of beams, whose mutual coherence can then be deduced from polarization measurements. A general Erouramatical description of such systems is developed, and several sxamples are examined in deta1l.

An estimate $15 \mathrm{glven}$ of the no1se level expected in the output of a multiple-beam spectroneter. The effect of photon no1se Is anolyzed and a criterion obtained for the amount of light required for acceptable photon statistics.

The results of an experimental study of spectrometers of thls type are presented. Several multiple-beam spectroscopic systems were assembled and tested with light from a small gas laser. The polarization fringe patterns obtained agree with those predicted by the tueory.

The final system studied was then used to observe fluctuations in a laboratory plasma. The plasma was produced in hellum by an electron beem, the fluctuations vere 1mposed upon it with a probe, and a selected wavelength and frequency component of the disturbance in the plasma was observed through measurements of I ictuations in the c'stributions of the sources of the strongest 
neutral helium emission line. The plasma phenomena observed were not extensively explored, but the results obtained show that a multiple-beam spectrometer can ectually be used for plasma diagnosties.

The theory developed to describe these messurements is then extended to some other cases. The se include the use of higher nrder optical correlation megsurements to detect higher order correlations in the source, the use of several optical frequencies to observe high-frequency phenomena within a plasma, and the use of a broad portion of the optical spectrum to make one observation more efficiently. 


\section{TNTRODUCTION}

\section{A. Optical Diagnostic Techniques}

Optical diagnostic techniques are used in nearly every field of physics. Progress made in recent years in optics ${ }^{1,2}$ has led to the use of improved optical methods in many areas of research. The purpose of this investigation was to explore the possibility of extending the optical methods which are used in experimental plasme physics.

In plasma phystcs, much effort has been Invested in the development of diagnostic techniques. ${ }^{3}$ Plasma diagnostics is particularly difficult because the phenomenn of interest occur during short times and becaluse a high-temperature plasma is easily perturbed by almost any instrument. A proke is required which can respond quickly--at high frequencies--lut which will not disturb the plasma under study. Both of these requirements suggest the use of optical methods.

Several oytical diagnostic techniques are useci in plasma physics. Tihe variety of existing methods calls for a discussion of the general problem of an interacting system of optical radiation and plasma. Such an analysis suggests that other useful optical techniques could be developed, one possibility, "multiplekean spectroscopy", is discussed in this report.

The besis of this approsch ${ }^{4}$ is the measurement of the coherence, or correlation in phese, between various conponents of the light emitted by a plasma. Analysis shows that such a measurement should provide information about local values of the fluctua- 
tions or correlations in the distribution of light sources within a plesma. To explore the practicality of using this technique for diagnostics, a model optical system was constructed arid used to observe imposed Pluctuations in the density of a helium plesma. produced by an electron beam.

Before explaining these ideas further, it is useful to review the basic principles and limitations of some standerd optical plesma diagnostic techniques. This is done next and then the concept of the present study is presented in the second chepter.

Note added in proof: All assembled, the report is longer than anticipated. A few comments on its structure may be helpful. The centrel portion of the text is sect. IIA. Chapter I is Just pisiminary to IIA, and later sections all stem from that sasic arguent. in particular, Sect. IIB (with Appendix C), Sect. IIC, Chapter III, and chapter V are four completely separate discussions, all of which directly follow sect. IIA.

The experimental work is described in Chepter IV. This should be understandable if one has read IIA ead then Appendices $C$ and $D$.

\section{B. Plasme Spectroscopy}

Any optical diagnostic technique Involves an interacting system of rediation and plasma (actuelly, any extended source of light would suffice for this analysis). (See Fig. I-1.) The plasma is assumed to be bounded, but many optical wavelengths 


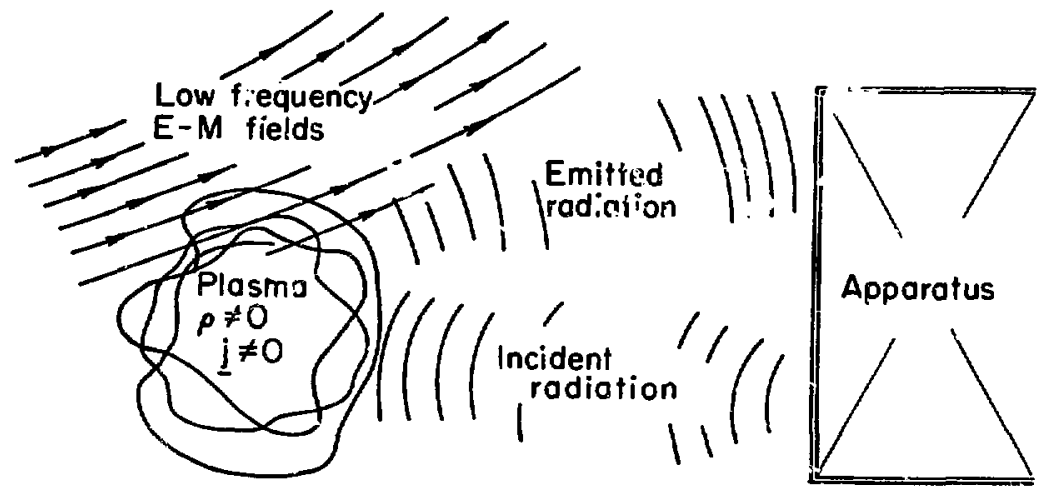

XEL $733-2397$

F1E. I-1. The general type of system considered. 


$$
\nabla_{\underline{E}}-\frac{1}{c^{2}} \frac{\partial^{2} \underline{E}}{\partial t^{2}}=4 \pi\left(\nabla \rho+\frac{1}{c^{2}} \frac{\partial \underline{J}}{\partial t}\right)
$$

and $\underline{B}$ obeys a similar equation. Thus, for the flelds themselves, one has again a set of equations of the form of (I.3).

In a system like that of Fig. I-l, the source density, $s(\underline{r}, t)$ is nonzero only within the plasma, but $\xi(\underline{r}, t)$ extends beyond the source. This, of course permits optical diagnostics: The optical frequency components of $\underline{\xi}(\underline{r}, t)$ (which may include Incident, as well as emitted or scettered l1ght) are observed-outside the plasma. This provides, according to Eq. (I.3), some degree of information atout the optical frequency conponents of $s(\underline{r}, t)$. And $s(\underline{r}, t)$ depends upon various properties of the plasma. In zelterai, $s(\underline{r}, t)$ depends also upon the fielas, including those of light waves. This effect of the light must be considered, for example, to explain scattering and to compute the index of refraction of the plasme.

In some situations, however, the effect of the light nay le neglected. The optical radiation may then be considered separately, using Eq. (I. 3) with a specifled $s(\underline{r}, t)$. ihis may be done if the light is emitted in collislons or atomic transitions and propagates unaffected by the plasma. We conoider first such an $s(\underline{r}, t), A$ transporent extended source, with $n=I$ ( $n$ is the index of refrection).

There are, then, three elements: Tile plasma, the optical raciation, and the apperatus of measurement. To explain a particular ctservation, one can calculate the effects of various 
plasma phenomena. But to determine what apparatus to use it is necessery to first consider carefully the nature of the optical radiation. What types of information are contained in the light from such a source? And what types of observation might one make? To measure the optical radiettion one can simply photograpin the plasma. ${ }^{6}$ But photographs alone can only begin to describe plasma phenorena and a measure of the total intensity gives only a small pert of the information in the emitted light.

Much more information is contained in the spectrum of the 1ight. ${ }^{7}$ The light from a plesma consists of line radiation from atoris and ions and continuum radiation dLe mainly to bremsstrahIung and cyclotron emission. 8 iti a spectrometer (F1g. I-2) one can compare the intensities of rarious portions of the spectrum and measure the shapes and locations of spectral lines. Since several mechanisms, including the Dol pler effect and the Stark effect, can broaden spectral lines, several piasma parameters may be determired spectroscopically.

Tbe accuracy of a spectroscoplc measurement is limited by the intensity of the avallable 21ght. Th1s, of course, is true of uny optical technique. In addition to this, a spectrometer like that shown in Fig. I-2 has two inherent limitations not necesserily shared by other optical diegnostic apparatus.

First of all, the various components of the light are emitted from small discrete source ${ }^{--\theta t o m s, ~ l o n s, ~ e l e c t r o n s, ~ c o l l i d i n g ~}$ particles, etc. Such light contains information about the source and 1ts Imnediate vicinity--the velocity of the source, the local 


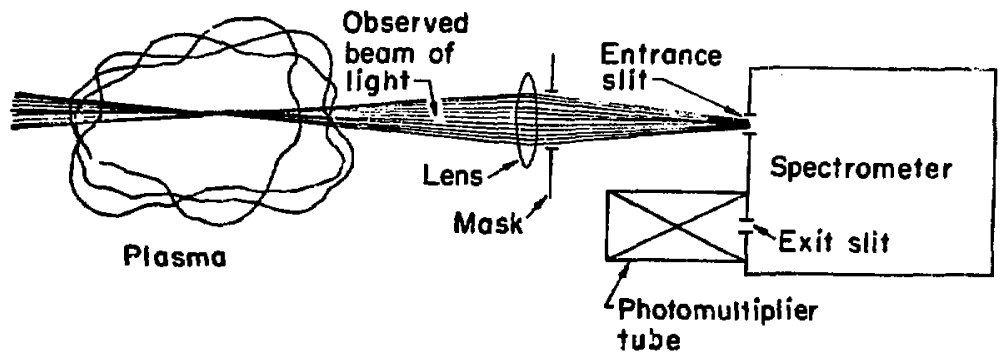

$X 8 L 7210-4328$

F1g. --2. Conventional spectroscopic apparatus. 
Plectric field, etc. From the spectrum, which is merely the superposition of such contributions, one can determine "single polnt" plasma parameters such as particle densitles and temperatures, and fleld strengths and frequencies. Plasma dynamics, however, is dominated hy collective effects due to long range forces by which particles some distance apart may interact. WithIn the confines of the observed beam, ${ }^{9}$ it is impossible to measure with an apparatus like that in Fig. I-2 such "plasma" properties as the wavelengths and frequencles of density fluctuations, or shlelding distances, or correlation lengths.

The second limitation is the lack of depth perception. A spectrometer like that in F1g. I-2 accepts light from sources within an observed keam. A typical focused beam is shown in Fig. I-3. For sources not too near the focus and well within the team, the optical system accepts any light emitted along a ray which when traced back goes through the focal spot. Such rays are apread over an angle $b \approx \delta / x$, that is, a solid ansle $-i^{2} \approx \delta^{2} / x^{2}$ ( $\delta$ = focal spot $\varepsilon 1 z$; $x=$ distance to focus). The radius of the observed region is $r \approx \frac{1}{2}$ ax ( $a=$ angular spread of the beam). So, the amount of light recelved from some element along the line of Bight (see Fig. I-3) 18

$$
\begin{aligned}
& \text { Intensity }=\left(\begin{array}{l}
\text { brightness } \\
\text { of source }
\end{array}\right)\left(\begin{array}{l}
\text { volume } \\
\text { of region }
\end{array}\left(\begin{array}{l}
\text { soild angle } \\
\text { subtended }
\end{array}\right)\right. \\
& =\left.\left(\begin{array}{l}
\text { brightness } \\
\text { of source }
\end{array}\right) \pi_{i} \frac{a x^{\prime}}{2}\right|^{2} \Delta x \mid \frac{s^{2}}{2} i^{2} \\
& =\begin{array}{l:l}
\text { ibrightness } & \pi \\
\text { of source }
\end{array} ; a^{2} \delta^{2} \Delta x
\end{aligned}
$$




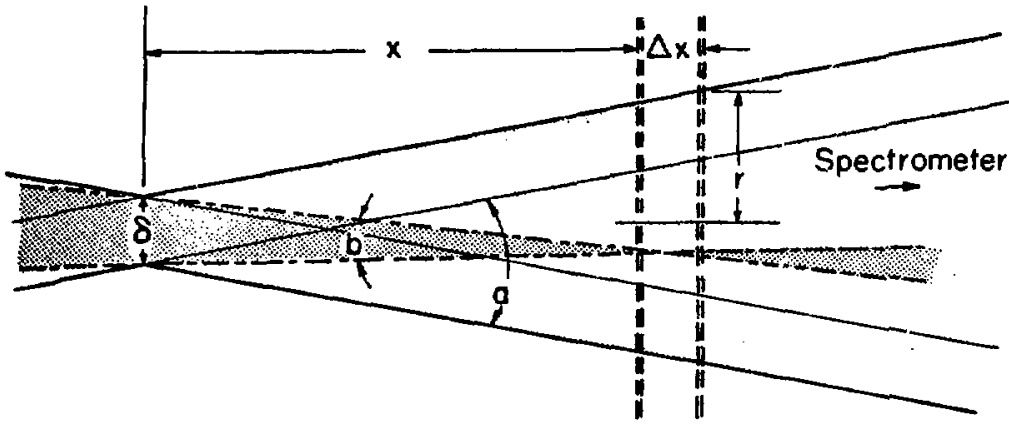

XBL-733-2398

Fig. I-3. A typical observation region (single focused beam). i Is the width of the focus, a is the angle of accertance at the focus, $b$ is the angular wiath of the focus as seen from a point a distance $x$ away from the focus. 
--Independent of $x$, except through the brightness of the source. So, at lesist according to this simple, approximate analysis, there is no spatial resoluiton at all in the exfal direction.

This conclusion is valid within geometricel optics. The intensity of optical rediaifon (the energy per unit solid angle crossing unit area in unit time, i.e., the energy flux per solid angle) is not chenged by an notical system free of losses and aberrations. ${ }^{10}$ This is just the result, familiar in photometry and photcrraphy, that the apparent brightness of a scurce depends upon its actual brightness, but not upon 1ts distence from the observer.

However expressed, Invariance of Intensity means that any measured optical spectrum is an unweighted average of spectra of light emitted all slong the line of sight. ${ }^{11}$ Ir the source is nonuniform, different regtons with different emission spectra contribute to every observation. Some type of "unfolding" is required. For th1s one must record spectra of light emitted along many different lines of sight. An additional assumption, such as cylindrical symmetry of the plasma, is generally also invoked to simplify the analysis.

Nevertheless, plasma spectroscopy has been found extremely useful. An optical spectrum contains a large amount of information. Interpretation of various spectral features can become quite complicated and present understending is besed on work by many investigators.

Explanation of the optics of the spectrometer, on the other 
hand, is quite straightforward when an idealized instrument, free of lens aberrations, misalignuents, etc., is used as a model. The apparatus depicted in Fig. I-2 messures the intensity of vari.. ous frequency cowponents of the light in soine bundle of rays. The frequency (more precisely, the wavelength) is selected by the spectrometer (slits, mirror and difraction grating) and the intensity is measured with a photomultiplier tube.

A light wave, even if a simple scalar model, is characterized by intensity, frequency, and phase. The spectrometer makes use of phuse Information to define the incldent bens. Different spectrometers select ifferent spectral lestures, but all conventional Instruments make similar use of the phase of the incldent 1ight: A lens or set of lenses and a pinhole or slit are used to select a bundle of rays--a result which can be deseribed by ge unetrical optics.

c. The Use of Coherent Light: Inverferometry and Light Scattering Measurements

There are optical diagnostic methods which do make different use of phase information. Within the last decade, optical interferametry and light Bcattering mes surements have both become widely used ... plow physics. These techniques differ from spectroscopy In toat light from an external source is used and in that the light interacts with the plasma: affects $s(\underline{r}, t)]$.

In spectroscopy, the intensity of the light used is the sum of the Intensities of components fro different sources. In interferometry and in scattering, the oiserved intensity of the 
light depends also upon the relative phases of varlous components. This is true because this light is coherent. It originates from a common source and its coherence length exceeds any differences in opticel path.

Opt1cal Interferometry is useful in studles of dense plasmas such as thete pinches. In such an observation, interference 16 used to measure the phase of light which has traversed a plasma. Th1s pinse depends upon the path of the light and upon the plasma Index of refraction, which, in most experiments, is determined meinly by the electron density. This effect is analyzed in the review by Jahoda and Sawyer ${ }^{12}$ who show that, at a given optical Hrequency, the expected phase shift is proportional to the integral of the electron densitj along the path of the IIght--again, a nonlocol teesurement of a single point parameter. For ruby laser light, an integrated density of $3.2 \times 10^{17} / \mathrm{cm}^{2}$ is needed to change the optical path length by one wavelength. For plasmas much smaller or less dense than this, zero phase shift is a good epproximation unless phase is meesured very precisely or light crosses ihe plasma many times.

The present analysis assumes throughout that the index of refraction of eny plasme consldered is equal to unity. This assumption is ade to simplify the analysis, tut it is not necessarily a general ilmitatical since in mar; cases some variation in $n$ would be inconcequential.

Meny il:terferometric technqques, including some which can be used to aeesure very small phase shlfts, have kaen developed. 
These methods are well sumarlzed in several reviews. 12,13

A different type of information is provided by studies of the scistering of eleatronagnetic raliation by a plasma. This technique was first used in radar backscattering studies of the 1onosphere. ${ }^{14}$ Therearter, theoretical analjses by several authors 15 . explained such scattering in terms of predicted fluctuations in the plasma electron density. Iaser ilght scattering has since been used to study a variety of laboratory plasmas. ' 6 Because an analysis of this type of measurement 1 is similar to less familfar protlems considered in later chapters, a brief discussion of this by now well-known technique appears $1 \mathrm{r}_{1}$ icated in this place. The apperatus for a typical scattering measurement is shown in Tig. I-4. The vasic procedure is simple: A laser illuminates the plasma and the light scattered int. sone otserved beam is spectrally analyzed. The measured spectrum is found to differ from that of the incident laser light because components of the scattered light are shifted in frequency by amounts comparable to varlous characteristic frequencles of the plasma. To understand the scattered spectrum--indeed, to understand why scattering occurs at all--one must consider the combined effect of scatterIng by many plasme particles.

The observed scattering occurs within the intersection of the incident and otserved keans. In this region the incident light may be represented as a linearly polarized monochromaitc plene wave: ${ }^{17}$

$$
\underline{E}_{1}(\underline{r}, t)=\underline{E}_{0} \cos \left(\underline{k}_{1} \cdot \underline{\underline{r}}-\left|\underline{k}_{1}\right| c t\right) \text {. }
$$




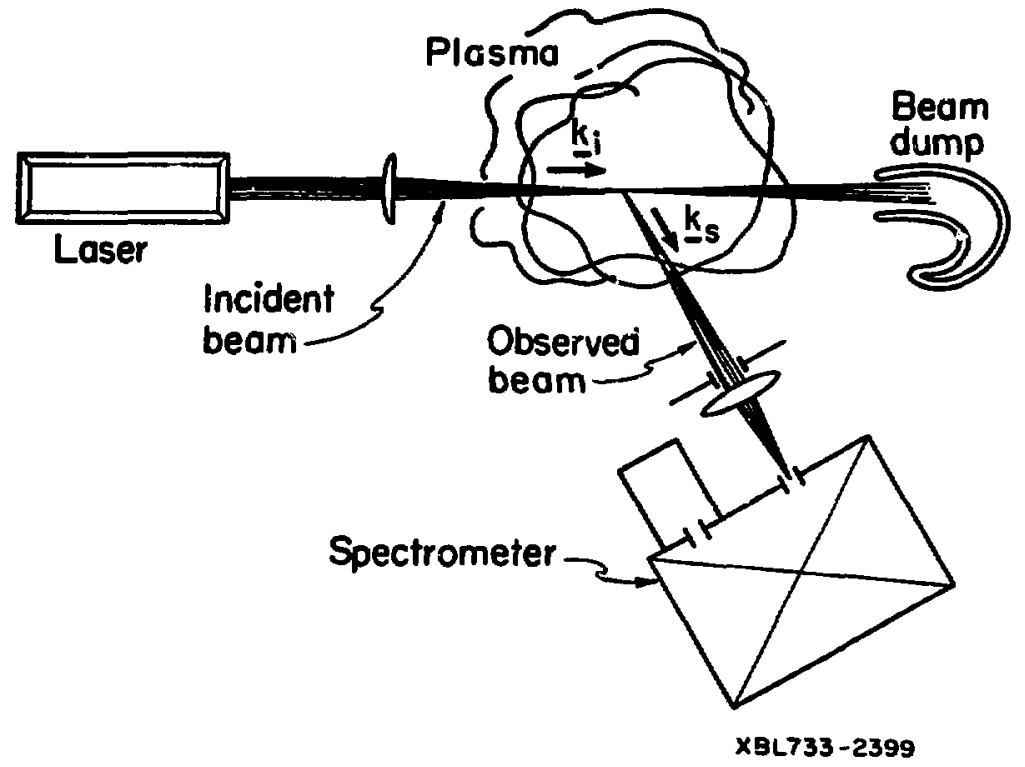

F1g. I-4a. Typical laser light scattering apperatus. $k_{1}=$ wave vector of the incldent 11ght, $\underline{k}_{8}=$ wave vector of the observed scattered light. 


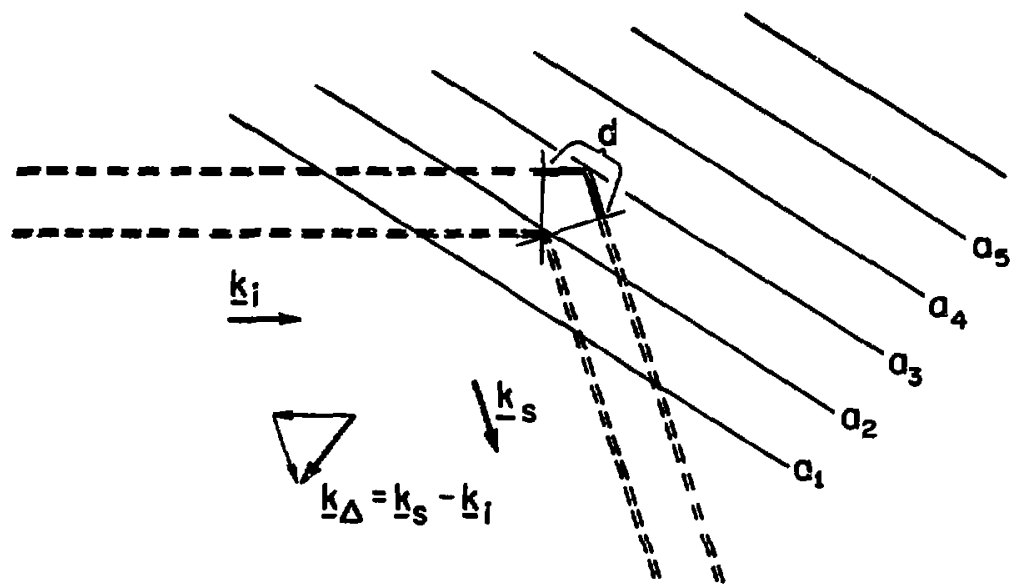

XBL733-2400

Fig. I-4b. Detall of the scattering region, $a_{2}, a_{2}, a_{3}, \cdots$ are the maxima of a wave of wave vector $k_{\Delta}$. d is a difference in path of one optical wavelength. 
Each charged particle is accelered by this field, e $=\frac{e}{n} \underline{E}_{1}$ and emits a scattered wave, $\Xi_{s}$. The total scattered wave is the superposition of such contributions. 18

For nonrelativistic motion, the radiation electric fleld of an accelerated point charge is: 19

$$
\begin{aligned}
& \underline{E}_{s}(\underline{r}, t)=\left.\frac{e}{e^{2}} \frac{1}{\left|\underline{r}-\underline{r}^{\prime}\right|} \cdot\left[\hat{n} \times\left(\hat{n} \times \underline{s}^{\prime}\right)\right]\right|_{\text {ret }} \\
& \hat{n} \equiv \frac{\underline{r}-\underline{r}^{\prime}}{\left|\underline{r}-\underline{r}^{\prime}\right|} .
\end{aligned}
$$

$\underline{r}^{\prime}$ and $\underline{a}^{\prime}$ are the position and acceleration of the particle at the retarded time

$$
t^{\prime}=t-\frac{l}{c}\left|\underline{r}-\underline{r}^{\prime}\right|
$$

Since the acceleretion, and hence the scattering, is inverseiy proportionsl to particle mess, appreciable scattering is produced only by plasma electrons. If these are described by an electron density $n_{e}(\underline{r}, t)$, then from a voivme $d^{3} r^{\prime}$ around a point $\underline{r}^{\prime}$ is emitted a scattered wave,

$$
\underline{E}_{B}\left(\underline{\underline{r}},+; \underline{\underline{x}}^{\prime}\right)=\frac{e^{2}}{m c^{2}} \frac{1}{\left|\underline{\underline{r}}-\underline{\underline{r}}^{\prime}\right|} n_{e}\left(\underline{\underline{r}}^{\prime}, i^{\prime}\right) \cdot\left\{\hat{n} \times\left[\hat{n} \times \underline{E}_{i}\left(\underline{\underline{r}}^{\prime}, t^{\prime}\right)\right]\right\} \text {. }
$$

And the total scattered wave is the superposition,

$$
\underline{E}_{s}(\underline{r}, t)=\sum_{\substack{\text { scattering } \\ \text { volume }}}^{d^{3} \underline{r}^{\prime} \underline{E}_{s}\left(\underline{r}, t_{j} \underline{r}^{\prime}\right)}
$$


This wave is analyzed by a spectrometer which transmits onl Is component (see Appendix E.I),

$$
\begin{aligned}
& \underline{E}_{s}\left(\underline{k}_{s}, t\right) \equiv \int d^{3} r e^{-i \underline{k}-s \cdot \underline{r}} \underline{E}_{s}(\underline{r}, t) \\
& =\frac{e^{2}}{m c^{2}} \int d^{3} r e^{-i \underline{k}-\underline{r}} \int d^{3} r^{\prime} n_{e}\left(\underline{r}^{\prime}, t-\frac{\left|\underline{r}-\underline{r}^{\prime}\right|}{c}\right) \\
& \cdot\left[\hat{\mathrm{n}} \times\left(\hat{\mathrm{n}} \times \underline{E}_{0}\right)\right] \frac{1}{\left|\underline{\mathbf{r}}-\underline{\underline{r}}^{\prime}\right|} \\
& \cdot \cos \left[\underline{\mathbf{k}}_{i} \cdot \underline{\mathbf{r}}^{\prime}-\omega_{i}\left(i,-\frac{\left|\underline{\mathbf{r}}-\underline{\underline{r}}^{\prime}\right|}{\mathrm{c}}\right)\right]
\end{aligned}
$$

$\left(\omega_{i} \equiv\left|\underline{k}_{i}\right| c\right)$. This expression reduces to (see Appendix $B$ for details),

$$
\begin{aligned}
& \underline{E}_{s}\left(\underline{k}_{s}, t\right) \underset{t \rightarrow \infty}{\rightarrow} \frac{-i \pi}{\left|\underline{k}_{s}\right|}\left|\frac{e^{2}}{m c}\right| \underline{E}_{0}^{1} \\
& \cdot\left\{e^{-1 \omega_{s} t}\left[n_{e}\left(\underline{k}_{s}-\underline{k}_{i}, \omega_{s}-\omega_{i}\right)+n_{e}\left(\underline{k}_{s}+\underline{k}_{i}, \omega_{s}+\omega_{i}\right)\right]\right. \\
& \left.+e^{-i\left(-\omega_{s}\right) t}\left[n_{e}\left(\underline{k}_{s}-\underline{k}_{i},-\omega_{s}-\omega_{i}\right)+n_{e}\left(\underline{k}_{s}+\underline{k}_{i}, \omega_{i}-\omega_{s}\right)\right]\right\}
\end{aligned}
$$

$$
\left(\omega_{\mathrm{s}} \equiv\left|\underline{\mathrm{k}}_{\mathrm{s}}\right| c\right) \text {. Here } \underline{E}_{0}^{1} \equiv\left(I-\hat{\mathrm{k}}_{\mathrm{s}} \hat{\mathrm{k}}_{\mathrm{s}}\right) \underline{E}_{0} \text { is the component of } \underline{E}_{0}
$$

which is normal to $k_{\mathrm{s}}$ and

$$
n_{e}(\underline{k}, u) \equiv \int j d^{3} r d t e^{-i(\underline{k} \cdot r-c t t)} n_{e}(\underline{r}, t)
$$

is the Fourier transfarm of the electron density.

If we retain only positive Irequency components of $\mathrm{E}_{\mathrm{s}}$ (see Appencix E.I) and reglect the high-îrequency $\left(\alpha=\omega_{s}+\omega_{1}\right)$ con- 
ponents of $n_{e}, E q .(I .7)$ reduces to

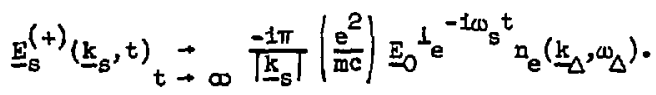

Here,

$$
\begin{aligned}
& \underline{k}_{\Delta} \equiv \underline{k}_{s}-\underline{k}_{1} \\
& \omega_{\Delta} \equiv \omega_{s}-\omega_{1} .
\end{aligned}
$$

This analysis neglects the width, $\Delta \mathrm{k}$, of the spectrometer Instrument function. With equal precision, the long time IImit may be replaced by equality after $t \sim \tau=(c \Delta k)^{-1}$, the correspondIng correlation time. This retains a slow time defendence in $\mathrm{n}_{\mathrm{e}}$

$$
\left.\underline{E}_{s}^{(+)}\left(\underline{k}_{s}, t\right) \cong \frac{-1 \pi}{\left|\underline{k}_{s}\right|} \mid \frac{e^{2}}{m c}\right) \underline{E}_{0}{ }^{1} e^{-1 \omega \omega_{s} t} n_{e}\left(\underline{k}_{\Delta}, \omega_{\Delta} ; t\right) .
$$

We thus introduce a time-dependent spectrum. Th1s operation is considered more carefully in Append1x E.3. The measured light Intensity, which depends upon $\underline{E}^{(+)}\left(\underline{k}_{s}, t\right)$ (see Appendix E.1-3) exhlbits only this slow time dependence:

$$
\begin{aligned}
I\left(\hat{k}_{s},\left|\underline{k}_{B}\right| ; t\right) & =\left(\frac{\left|\underline{k}_{s}\right|}{4 \pi^{2}}\right)^{2}\left|\underline{E}^{(+)}\left(\hat{k}_{E},\left|\underline{k}_{E}\right|, t\right)\right|^{2} \\
& =\frac{1}{16 \pi^{2}}\left|\frac{e^{2}}{m c}\right|^{2}\left|\underline{E}_{0}\right|^{2} \mid n_{e}\left(\underline{k}_{\Delta},\left.\left(\omega_{\Delta} ; t\right)\right|^{2} .\right.
\end{aligned}
$$

The observed scattering is thus due to one Fourier component, the $\left(\underline{k}_{\Delta^{\prime}}, \omega_{\Delta}\right)$ component, of the electron densfty. A single electron would produce a scattered wave, but when many electrons are present, 
only fluctuations in their density will cause scattering. This can be simply explained. All light scattered by electrons located in a plane normal to $k_{\Delta}$ will have the same phese. Contributions from scatterers separated by $2 n \pi \hat{k}_{\Delta}\left|k_{\Delta}\right|^{-1}$ will differ in phase by a cycles. If $n$ is an integer, there will be constructive interference; if $\mathrm{n}$ is half integral, the contributions will cancel. If the electron distribution is unform, there will be complete cancellation. But any flucturtions $n_{e}\left(k_{\Delta}, w_{\Delta}\right)$ h:ll produce very strong scattering. [The scatterea intensity is proportional to the square of $\left.\left|\ln _{e}\left(k_{\Delta}, \omega_{\Delta}\right)\right| \cdot\right\}$

A plasmer wave can produce such fluctuations. Indeed, a description of scattering may be included in a more general analysts of three-wave interactions. In this context the resonance conditions $k_{\Delta}=\underline{k}_{s}-\underline{k}_{1}, \omega_{\Delta}=\omega_{s}-\omega_{1}$ are seen as statements of the conservation of momentum and energy. ${ }^{20}$ An analysis of scattering as a three-wave process may include the effect of the interaction upon $n_{e}$ and perhaps also upon $E_{1}$. These effects have been neglected here.

In conventional notation, the result of Eq. (I.II) 1s often expressed in terms of frequency,

$$
\begin{aligned}
I\left(\hat{k}_{s},\left|\underline{k}_{s}\right| ; t\right) d^{2} \hat{k}_{s} d\left|\underline{k}_{s}\right| & =I\left(\hat{k}_{s},\left|\underline{k}_{s}\right| ; t\right) d^{2} \hat{k}_{s} \frac{d \omega_{s}}{c} \\
& =\frac{c}{16 \pi^{2}}:\left.\frac{e^{2}}{m c^{2}}\right|^{2}\left|E_{0}\right|^{2}\left|n_{e}\left(\underline{k}_{\Delta}, \omega_{\Delta} ; t\right)\right|^{2} d^{2} \hat{k}_{s} d w_{s} \\
& =I_{0} n_{e O} \sigma_{T} S\left(\underline{k}_{\Delta}, \omega_{\Delta}\right) d^{2} k_{s} d \omega
\end{aligned}
$$


where

$$
\begin{aligned}
& I_{0}=\frac{c}{4 \pi} 2\left|\underline{E}_{1}^{(+)}(\underline{r}, t)\right|^{2}=\frac{c}{4 \pi} e\left|\frac{E_{0}}{2}\right|^{2}=\frac{c}{8 \pi}\left|E_{0}\right|^{2}, \\
& n_{e 0} \text { is the meen electron density, } \\
& \sigma_{T}=\left|\frac{e^{2}}{m c^{2}}\right|^{2} \frac{\left|E_{0}\right|^{2}}{\left|E_{0}\right|^{2}}
\end{aligned}
$$

Is the differential Thompson scattering cross section, and

$$
s\left(\underline{k}_{\Delta}, \omega_{\Delta}\right)=\frac{1}{2 \pi m_{e 0}}\left|\ln _{e}\left(k_{\Delta}, \omega_{\Delta} ; t\right)\right|^{2}
$$

Is celled the "dynamic form fector."

In a scattering experiment, one records a spectrum of the light scattered into some direction $\hat{k}_{s}$. The reiative variation In optical wavelength is usually negligible, so the scattering is alI due to Pluctuations of one wevelength, $2 \pi|k|^{-I}$. Th1s is customar1ly related to the plasma Debye length $\lambda_{\mathrm{D}}$ by a "scatterIne parrmeter",

$$
\alpha \equiv \frac{J}{\left|k_{\Delta}\right| \lambda_{D}}
$$

The spectrum of scatterea light then provides a frequency spectrum of the $k_{\Delta}$ corpponent of $n_{e}$. By the Wlener-Khintchlne theorer, ${ }^{2 l}$

$$
\frac{1}{2 \pi}\left|n_{e}\left(k_{\Delta}, w\right)\right|^{2}=\int d \tau c^{L \omega r} \overline{n_{e}^{*}\left(k_{\Delta}, t\right) n_{e}\left(\underline{k}_{\Delta}, t+\tau\right)},
$$

this 1s equivalent to a measure of the time correlation function, 


$$
c_{n_{e}\left(k_{-}\right)}(\tau) \equiv \overline{n_{e}^{*}\left(k_{\Delta}, t\right) n_{e}\left(k_{\Delta} s t+\tau\right)} .
$$

A complete knowledge of $n_{e}(\underline{k}, \omega)$ would also provide the complete spatial correlation function, but thls would require many observations. A single measurement provides partial--yet extremely useful--information about spatial correlations.

Scattering measurements are very useful precisely because they are not subject to eltiner of the prevlously noted limitations of spectroscopy. A spectrum of scattered light is not an average along a line of sight. The observed scattering occurs entirely within the intersection of the incident and observed beams. This well lacallzed scattering volume may be selected at will. And the measured correlation function is not a singlepoint farameter. The interference vetween light scattered from different points provides information about fine-scale fluctuations within a plasma.

W1th Eq. (I.II) one can deduce, frow an opt1cal measurement, a spectmun of electron density fluctuations. This result may then be compared with calculations of predicted spectra. Considerable effort has been invested in this type of study. The measured spectrum is found to depend strongly upon $\alpha$, the scattering parameter. For $\alpha \ll 1$, the observed fluctuations are those of a random distribution. In this regime the frequency spectrun is determined by the electron velocity distribution. $2 \bar{c}$ If $\alpha>1$, electron-1on correlations permit observation of 1on motions as well. 23 
Celcuiations of predicted fluctuation spectra differ in method and in assumptions, but the relation of such results to any scattering measurement depends upon opticai conglderations which are comon to all such experiments. In this report we consider further this fingt part of the problem--the relation between a distributed source such as a plasme and the assoctated optical radiation. It will be seen that various aspects of the foregoIng analysis are not unique to scattering.

\section{Information in the Emitted Light}

Consider further the general system of Fig. I-1 . The simplest such situation 1s, again, a self-luminous plaswa. In the optical problem, the source distribution $s(\underline{x}, t)$ is then determined by the various plasma processes. We assume for now that the plasma is an incoherent source. If one range of frequencies is considered, there is no correlation between the phases of $s(\underline{r},(0)$ at different points.

The resulting radiation, however, is not completely incoherent. Components of the light at differeni polnts are due to cammon sources and therefore the light $\xi(\underline{r}, t)$ observed at widely separated polnts may well be correlated in its phase. If the correlations are considered, an analysis of the radation from a luminous plasme is far from trivial. And such an anglyais presents the posibility of developing useful optical diagnostic techniques.

The bas1s of "multiple-beam spectroscopy" is the fact that information about the local values of fluctuations and correla- 
tions in plasme particle densities is in fact present in the light emitted by the plasma itself. The abcre noted linitations of apectroscopy are not limitations on the information contained In the emitted light. They are limitations of the type of apparatus represented In Fig. I-2. To make a different type of measurement, one must make differe ut use of the phase of the light.

Although these conclusions can be justifled by a general analysis, they were first obtalned by consit.. ration of particular opt1cal systems. This approach has been retained in the explanetion which follows. We first describe a simple two-beam spectroneter and then consider the possiblities and the difficulties ouggested by the new arrangement.

The development of "multiple-beam spectroscopy" was based upon experience with a scattering experiment and this is reflected In the following explanation. Laser light scattering methods are famili:-r to plama physlc1sts, but a scattering measurement is not the only optical technique which uses phase information in way which cannot be explained by geometrical optics. In particular, the Invention of the laser has also led to the practical development of optical holography. 24-26 A "hologram" is a recorded set of interference fringes which can be usea to produce a threedimensional image of a stationary object. In a conventional hologruphic process, interference w1th the ifght in a reference beem is used to produce a record of the amplitude and phase of the light reflected by a coherently illuminated object. An explanation of this procedure bears conside:able simllarity to an analisis 
of laser light scattering.

More recentlij, the poseibllity of making holograms of self Iuminous objects, or of incoherently 11luminated objects, has also been explored. 27-31 This work is of interest in connection with the present study. The relation between lolograph; and optical plasme diagnostics is examined in Appendix $A$ of this discussion. In the rext chapter, multiple-beam spectroscopy is explained in terms of classical optics. Some consequences of the diacreteness of light ywanta are discussed in Chapter III. 
II. THE UTE OF PHASE THFORMATION

A. A Two-Beem Srectrometer

1. Light from an Incoheren: Source: The Sum of Kany

Interference Patterns

Every optical phase measurement requires a comparison between different components or beams of light. In interferametry, a recorded pattern of interference fringes reveals the difference in phase between transmitted light and light in a reference beam. In a scattering process, the interference between light scattered by different electrons depends upon the coherence imposed by the light In the incident beam.

A self-luminous plasma provides no incident or reference beam, but eince plasma radiates in all directions, the emitted light may be considered to consist of many beams. Light from different points within a plasma 1s, in general, not coherent, but light enitted in different directions from one region should have sorae coberence. Thus, if a volume of plasme were observed from several directions at once, both phase and frequency measuremen's could be made.

Most simply, one could define two distinct "observed beams"-$A$ and B--as shown in Fig. II-1. Beyond a spectral analysis, or any other measurement on either beam alone, there is then a further possibility: to compare the light in the two beams.

Th1s suggeate at once that a local spectroscopic measurement might be possible. The two nbserved beams can be defined to intersect in only a smell, well locallzed, cammon source volume. Then 


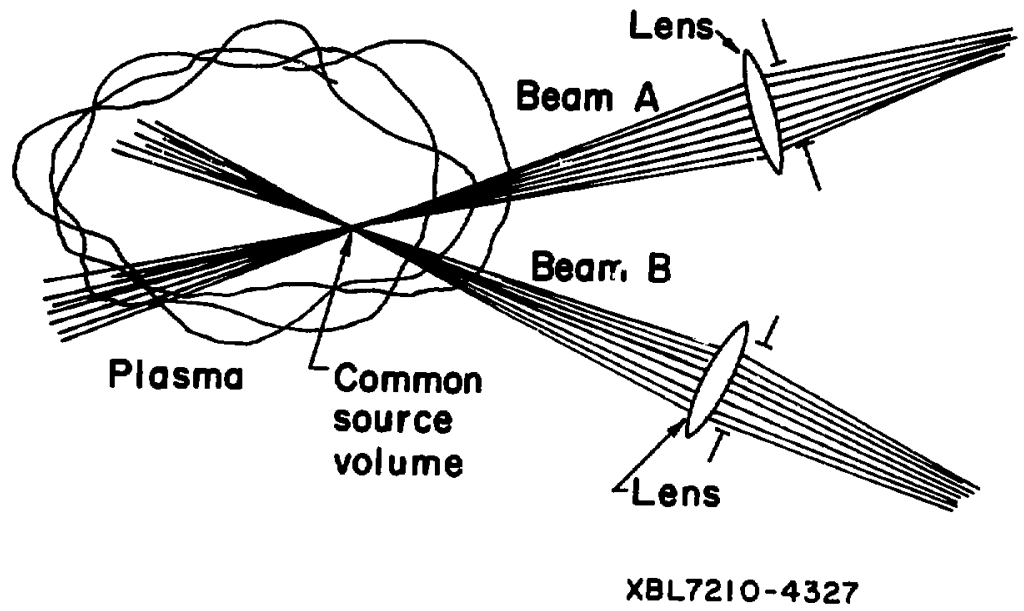

FIg. II-1. A two-beam observation. 
If any component of the light can be identified as conmon to both beams, the location of the source is known at once. In buman vision, for example, depth perception is provided by the recognition of two images of a single object. Thls procedure would be difficilt to duplicate with scientific apparatus, but in obsemations of a plasma, the very incoherence of the source provides another way in which a common optical component might be recognized. We have noted already that light from a conmon source is (or may be) coherent. If it is assumed that light from different sources in a plasma is completely incoherent, then any correlations In phase between the light in beams $A$ and $B$ must be due to common sources. Any measured mutual coherence between camponents of the Iight In different beams must give local information about the common region where the two beams intersect.

To measure the mutual coherence between beams $A$ and $B$, one cen combine them and observe any two-beam interference which results. A simple optical system with which this cculd be done is shown in FLg. II-2. Here a set of masks and lenses is used to define two narrow beams which intersect in a carmon source volume. The light In each beam goes through a filter (that is, through some spectroscopic apparatus, the same for each). We suppose that the olserved light contains a spectral line of width ${ }^{\prime}$, that the filter transmits only this line, and that the coherence length, $c / \Delta w$, of this transmitted light, exceeds any differences in the lengths of opt1cal pathe through the system.

Feams $A$ and $B$ are focused at a comon point on a screen. The 


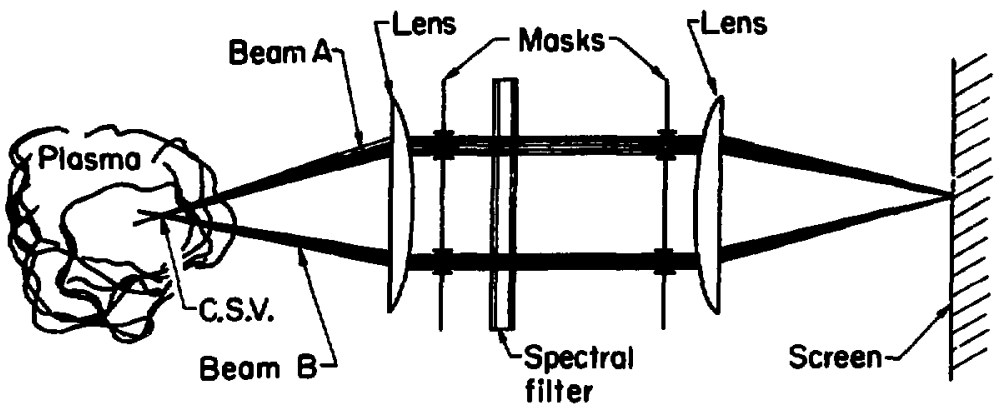

XEL $733-2401$

F18. II-2. A two-beam spectrometer. c.s.v. = common source volume. 
illumination of the screen then depends upon the mutual coherence of the light in the two beams. If the light were all due to a single point source which vas observed through only one of the beams, then a single aperture diffraction pattern vould appeur on the screen, as shown in F1g. II-3a. The width of the illuminated aree vould be determined by the angle $\phi$ subtended at the screen by a single beam.

If, however, the light were due to an lsotropic point source which was observed through both beams, the result would be quite ifferent. In this case, a two-beam interference pattern would appear on the screen, as shown In Fig. II-3b. The spacing of the fringes of this pattern would depend upon the angle $\theta$ between the two component beams. Since $\theta \gg \phi$, the angle subtended by a single beam, the fringe spacing would be much less than the size of the whole pattern and many fringes would be seen.

If several separate incoherent sources were observed at once, the resulting pettern of illuminetion would be simply the superposition of the light intensity distributions due to each of the sources alone, as shown in Fig. II-3c. Two sources, one observed through each beam, would not togetber produce a two-beam Interference pattern. Interference requires mutual coherence which, under our assumptions, could be provided only by a coumon source. These conclusions are based upon quite elementary optics, but the essential difference between the effects of sources which are observed through one beam and the effects of sources wh1ch are observed through both beams presents a practical useful possibility. 
(a)

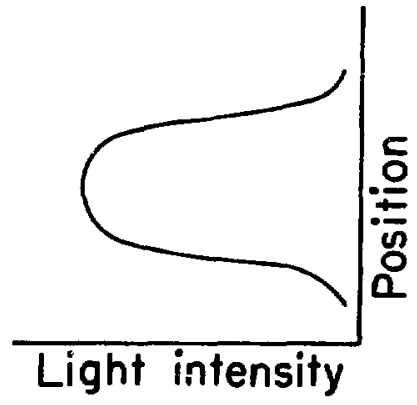

(b)

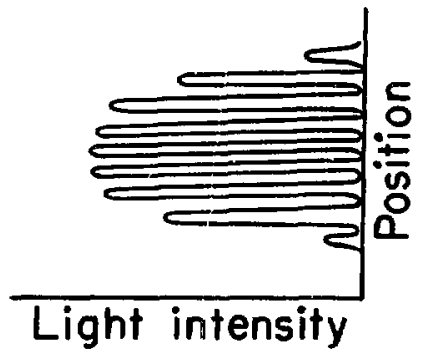

(c)

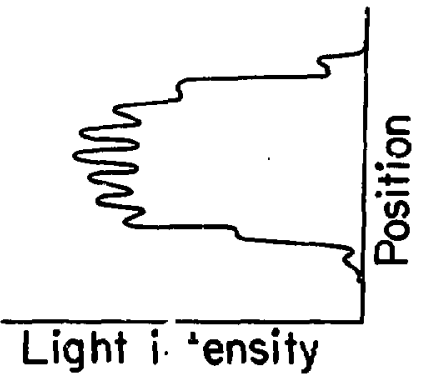

$X B L 733-2402$

F1g. II-3. For legend, see page 31. 
F1g. II-3. Patterns of illumiration of the sciets.

(a) A smooth distribution of light intens:ty iue to a source observed through one beam. (b) Two.nkeam interference fringes due to a source observed through boith beams. (c) A pattern due to several seperate solxees. 
If an optical system of this type, a two-beam spectrometer, were used to observe a plasma, and if the emplitude of the interference pattern, and not the total light intensity, were recorded as a spectral amplitude, the result would depend upoin cnly those sources within the small, well localized common source volume. In this manner, one could observe exnlusively a small selected region within a luminous volume of plasma. This is something which cannot be done with conventional spectroscopic epparatus.

The essential difference is that, in I single beam observation, unwanted light is stopped only by some system of masks and slits. Any light of the proper frequency which is admitted by the optical system--and this includes all the light radiated along certain rays--contributes to the output of the spectrometer. With two beams, however, it is possible to discriminate against a portion of thet light which is edmitted by the opticel system. This is what gives the better resolution.

In a laboratory instrument, it is useful to observe an optical signal electronically. In a two-beam spectrometer, there are everal ways in which this might be done. Most simply, the screet (In Fig. II-2) could i- replaced by an array of flits placed at the positions of the maxima and minima of an expected two-beam Iaterference pattern. Then a set of mirrors or light pipes could be uoed te direct the light from a set of maxima (which we shall call "beam 1") Into one photomultiplier tube ("tube I") and the light from the corresponding minime ("beam 2") into another phototube ("tube 2"). Then, if the illumination were uniform, the two 
measured Intensities would be equal, but if the expected two-beam interference pattern vere present, most of the l1ght would be recelved by one of the phototubes. One could then record, as the output of the system, not the total measured intensity, but rather the difference between the two phototube olgnals.

Any observation made with such an instrument would be a locel measurement. Light from a source observed only through beam A or only through beam $B$ would be divided equally between beams $I$ and 2 . Such IIght would not contribute to the recorded difference signal. Only light from common sources would (or might) be divided unequally between the two phototubes.

There is, $h$ wever, a further complication. The apparatus of Fig. II-2 defines a conmon source volume which is at least as wide as a diffraction-limited focus of elther of the two observed beams. To every polint within this region there corresponds an expected set of two-beam interference fringes on the screen. But the patterns due to different sources might not colnclde.

Indeed, it can be seen at once that all such patterns would not colncide. The locations of the maxima of such a pattern depend upon the difference between the lengths of the two uptical paths frow the source to a point on the screen. To estimate the effect of a displacement of the source, it is helpful to imagine interchangirg source and screen. If a screen were placed at the comwon source volume and a point source were placed where we have drawn a screen, a set of two-beam Interference fringes would agtin appear. The spacing of the fringes in this pattern would depend 
upon $\alpha$, the angle between the beams, and the size of the 1lluminated region would depend upon $\beta$, the angle subtended ly either beam (see Fig. II-2). Since $\alpha \gg \beta$, there would be many fringes in the pattern. Because the various optical paths are unchanged by the interchange of source and screen, the maxima of the new pattern are just the locations in the original arrangement of source whlch would have produced Intenslty maxima at the location of the new source. Such sources would have ell produced colncident sets of Interference fringes ("pattern 1"). Sources at intermediate points--the minima of the new pattern--would in the oris:nal arrangement have produced the oprosite or complementary twobeam interference patterm ("pattern $2 "$ ). Finally, the regiun 1lluminsted in the interchanged arrangement is just the original common source volume. Hence the multipliclty of fringes here Implies the presence, in the system first considered, of different points from which light would contribute in opposing fashion to the output signal. Some conmon scurces would radiate preferentially 1nto phototube 1; other comon sources would radiate preferentially into phototube 2 .

If the common source volume vere filled with luminous plasma, botl types of source could be txpected. The result might be a cancellation of effect. To understand the significance of this conclusion, it 1s helpful to recall some features of a laser light-gcattering exporiment. In that type of measurement, scatterers at different polnts necessarily contribute light of different phases to the observed bean and such contributions can 
destructively tnterfere. As we have noted already, a uniform distribution of scattering centers would produce no scattered wave at all. The scattering is due to pluctuations in the density of scatterers. More particularly, according to Eq. (I.II), the light scattered by plasma is due to one spatial Fourier component, the $\underline{k}_{\Delta} \equiv \underline{k}_{s}-\underline{k}_{1}$ component, of the electron density. Thus the partial cancellation of effect leads not to a null output but rather to a different and quite useful type of information. The two-beam spectrometer of Fig. II-2 would in fact produce a similar result. From within the common source volume, light emitted Into a narrow range of directions around $\hat{k}_{A}$ is accepted by beam A (see Fig. II-4). If a common source were displaced by 6, the optical path to the screen through beam $A$ would be reduced by $\underline{\sigma} \cdot \hat{k}_{A}$ and the change in phase along this path would be reduced by

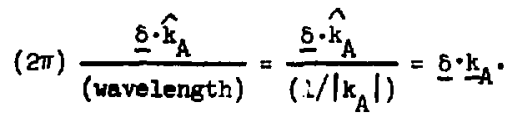

( $\underline{k}_{A}=$ wave vector, at the source, of the l1ght in beam $A_{1}$ ) The came displacement 6 would reduce the difference in phase from the source to the sureen along beam $B$ by $\underline{s}^{-} \underline{k}_{B^{*}}$. Hence the relative phase of the l1ght in the two beams would be changed by an amount

$$
\underline{8} \cdot \underline{k}_{B}-\underline{8} \cdot \underline{k}_{A}=\underline{8} \cdot\left(\underline{k}_{P}-\underline{k}_{A}\right)=8 \cdot \underline{k}_{\Delta}
$$

$\left(k_{\Delta}-\underline{k}_{B}-\underline{k}_{A}\right)$ which depends upon the displacement $\underline{s}$ and upon $\underline{k}_{\Delta}$ ' the difference wave vector, a quantity which appeared already in 


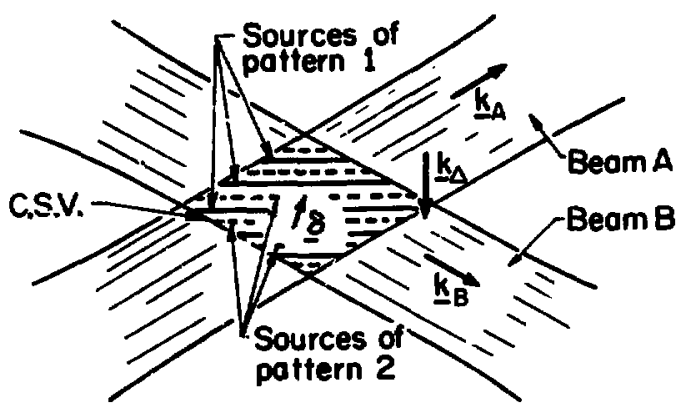

XEL733-2403

F1B. II-4a. The effect of a displacement of the source--detail of the source region. c.s.v. = compon source volume.

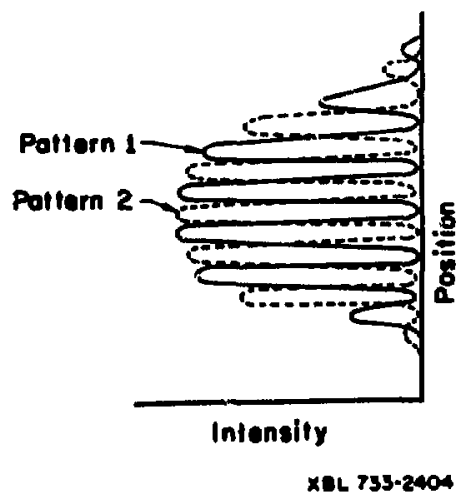

F18. II-4b. Correoponding patterns or fllumination or the screen. 
the analygis of scattering.

If the displacement $\delta$ were nomal to $k_{\Delta}$, there would be no change in the relative phase of the light in tre two beams. Hence all the common sources in a plane normal to $\mathrm{k} \Delta$ would produce coincldent interference patterms. Each such source plane would act as a single source. Furthermore, different source planes separated by an integral multiple of the distance $2 \pi /\left|k_{\Delta}\right|$ would also produce the same pattern ("patterm I"). But sources in an intermediate set of planes (see Fig. II-4) would all produce the opposite or complementary set of fringes ("pattem 2"). Hence the amplitude of the resulting two-beam interference--that is, of the mutual coherence between the light in the beams $A$ and B-would be proportional not to the total intensity of comon sources, but ratber to the difference in intensity ketween these two groups of saurces. This quantity is simply the amplitude of the $k \Delta$ spatial Fourier co ponent of the source distribution.

Here it should be emphasized that although this result is gimilar to what is found in an analybis of scattering, the reasons for the same effect are somewhat different in the two cases. The rosult of a scattering meagurement depends upon laterference between the light ecattered from different points within the scattering volume. The coherent incldent beam provides a definite relation between the phases of waves scatrered ly different electrons. Boause of the resulting interference, the measured intensity of scattered ilght depends upon fine scale fluctuations in the plasma electron density. 
To describe a spectroscopic observation, one must make the opposite assumption: The light from different sources is completely incoberent. There is no observable phase relation between the light emitted from one point and that from another polnt. In effect, two such waves would not interfere at all.

How then could one observe the source distribution? The answer 1 s that $1 f$ one looked from one direction only, one could not. But if a plasma were observed from two directions, with the Instrument shown in FHB. II-2, (1) each ecrion source would produce an interference pattern on the screen; and (2) if the sources were distributed in space according to $k_{\Delta}$, these various interference patterns would colncide. Then an overall patters would appear on the screen.

The light from each source would interfere only with itself, not with the light from another source. It is the relation between the different interference patterns which then gives information about the source distribution. But when one combines Interference patterns, one adds Intens1t1es; one does not add olectric flelds. In this arrangement, it is the superposition of the various Intensity patterns which corresponds to the interference of light waves (olectric flelds) in a scattering measuremont. 32 A two-bous spectroweter could, of course, be considered without reference to a scattering mosourement, but in any explanation it 18 important that points (1) and (2) should not be confused. Both otops are essential to the result.

The effect of the spatial distribution of sources has been 
discussed as if it were stationary, whlch it would not be for times longer than $\tau_{0} \approx I / w_{0}$, where $\omega_{0} 1 s$ a frequency character12ing the $k_{\Delta}$ component of the distribution. This affects the manner in which the slgnal must be recelved, but it also introduces the possibility of observing directly the frequencles characterizing the plasma.

We have assumed already that the observed light is a spectral Ine of width $\Delta$. It follows that the messured light intensities cannot vary at frequencies greater than this. The time resoluticn of the system 1s further limited by the respanse times of photomultiplier tubes. If it is assumed that the signal does not vary over times shorter then $\tau=\left[c^{-1}\right.$ (length of the common source volume)] then the output of the system at any instant represents the (single) Alstribution of sources at the time the light was emitted. In this low frequency limit, the output signal would reproduce d.rectly the (fluctuating) time dependence of the $k_{\Delta}$ component of the distribution of common sources. (This is shown In section II A 3 below.) If, for example, one had in the plasma a weve vector $k_{\Delta}$ and (low) frequeniy $\omega_{0}$, one would see in the signal an oscillation at $\omega_{0}$ (provided, of course, that the light souxce intensity varied with the amplitude of the plasma wave). Thet 1s, portion of the light accepted by the system would obclllate between beams $I$ and 2 at $\omega_{0}$, the frequency of the wave. In the usual spectroscoplc or light scattering measurement, It is oufficient to rocord an integrated intenglty value. The Pluctuation of the light intensity about the measured average is 
not usually considered. In the present case, however, the fluctuation is important because the measured signel is the difference between two light Intensities (beams 1 and 2). A long time average of this signal would produce a null result. It is essential to observe the signal over times less than $\tau_{0}=1 / \omega_{0}$, the fluctuation time.

There are several ways in which this might be done. For pulsed experiments, the optimal procedure would be difficult to specify in general, but in a steady-state experiment one could simply record a frequency spectrum $Y(\omega)$ of the output signal $Y(t) \equiv \overline{I_{2}(t)}-\overline{I_{1}(t)}$. The recordes $Y(\omega)$ would be proportional to the spectrum of the $\underline{k}_{\Delta}$ component of the common source (1ntensity) distribution. (see Section II A 3.) For example, a plasma wave (In the common source volume) of wave vector $\underline{k}_{\Delta}$ and frequency $\omega_{0}$ would produce a peak at $\omega_{0}$ in the recorded signal spectrum $Y(w)$. Finally, it should be noted that in several areas of research, methods are used which involve effects simflar to those considered bere. Interference between scattered waves is important, for example, in the scattering of x-rays by crystals ${ }^{33}$ and in Brillon scattering of light by sound waves in l1quids. ${ }^{34}$ Theoreticel analyses of the reflection of radar ${ }^{35}$ waves also lead to many of the same results.

The suggested use of two-bean interference to resolve fluctuations in light source densities within a plasma is quite simflar to the manner in which the diameters of stars can be measured with a Michalison stellar Interferometer. 36 
Another class of instruments whicb bear an interesting aimilarity to a two-bean spectrometer are the laser Doppler anemometers whlch are used to study gas and liquid flows. The literature on these devicee is extensive, but the most common types of leser anemometers are mentioned in one conference review article by Durst, Melling, and Whitelaw. 37 a Those authors describe three arrangements (see their Fig. 1). The first is just a simple scattering experiment, but one in which the scattered light is combined with light from the incident beam before detection. This permits measurement of small frequency changes, which produce beats in the observed intensity. In the flufd systems on which these anemometers are used, the particle positions are essentially random over distances $\left.\left.*\right|_{\Delta}\right|^{-?}$, so the scattered light intensity 1s just the sum of contributions from the different particles. (see section I C above.)

Durst et al. then describe anotber type of system in which only scattered light $1 E$ seen, but in which the scattering region is 1lluwinated with two beams from the same laser. The two incident bears interfore to give a pattern of fringes within the region observed. The scattered light is then found to te modulated by the wotion of fluid denelty fluctuations across this pettem of verying 1llumination. Tals system if similar to the inverse of a two-beam spectrometer: Instead of a two-beam observation, one bes two Incident beams, but In each case the two beams interfere to define a source vavelength, and in each case the observed Intonsity 1o just the en of contributions frow the different 
particles, in spectroscopy because of incoherence between sources ana in scattering because of randomness in particle position. So for different reasons, one obtains quite similar results in the two cases.

Finally, the same authors mention also a system In wh1ch only a single incident beam is used, but in which the scattering is then observed from two directions. This system is the one which most resembles our two-beam system. A two-beam system used to measure random scattering is clearly similar to a two-beam spectrometer. However, in the laser anemometer, only one final light intensity is measured, so the signal contains contributions from each beam alone, as well as a correction due to interference. So even in the absence of coherence there would be an output (as frow either tube 1 or 2 in our two-beam system) but in practice the effect of interference can be separated, since it gives a rapid time dependence to the measured gignal.

Moreover, one can also use an Intensity difference measurement to separate the interference in a laser anemometer. An arrangement which uses polarizing optics to do this has been studied by Bossel, Afller, and Meier ${ }^{37 b}$ in an experiment of the two-incident-beam variety.

\section{Same Comments on These Regults}

Thus a review of several videly used techniques fllustrates the importance of an understanding of the coherence properties of an optical rediation flold, and an analystis of a simple two-beam -pectrameter shows that inforwation about the local velues of the 
wevelengths and frequencles of fluctuations in light source densities is available, at least in principle, from measurements of the coherence of the emitted light. With this understanding, the present study was undertaken to explore the possibility of developing from these 1deas a useful, practical diegnostlc method.

Severel general features of this type of meesurement can be seen already from the first example. Only the effect of a single opectral line was described, but the same procedure could clzcrly be repeated for several portions of a spectrum. Since different spectral features are due to different types of sources, it should be possible to measure in this way the distributions in $\underline{k}$ and $\omega$ of various groups of particles. (In a scattering measurement, by comperison, only the electrons are observed directly.) With a two beam spectrometer, all of the information in the spectrum of the emftted light would still be arallable and one could measure at each optical frequency not only the total light intensity, but a whole set of optical correletions as well.

In a practical arrangement, the angle $\alpha$ between the beams could easily be mede small by observing the plasma through two sections of one lens. This would permit observation of plasma wavelengthi much larger than optical vavelengths. (Again, in comparison, a scattering study of long wavelength fluctuations requires the rather difficult observation of forward scattering.)

In comperison with the output of a conventional spectroneter, the level of the sigral from a two-beam gy atem would be much reduced. This, of course, simply roflects the improved resolution 
of the instrument: Only a fraction of the sources observed through elther beam contribute to the signal.

The problem is more serious, however, because in a real system, those sources which were observed, but which did not contribute to the signal, would produce a measured background nolse. In the classical optical picture the "background light" (not to be confused with "stray light", which can be reduced by improvements in the optics) is divided equally between beans 1 and 2 and the effect is balanced out in the intensity difference signal. In a real system, the tackground light would contribute an irreducible amount of photon nolse. The "signal" and "background" components differ simply in their photo count probability distributions and the seraration of effects is a problem in staristics. The need for adequate photon statisties thus imposes a basic light intensity (and observation time) requirement which is considered in Chapter III of this discussion.

The need for adequate light intensity leads one to consider improvements in the design of the optical system. The two-beem spectrometer so far considered is extremely inefficlent hasause the plasm is observed only through two narrow bundles of rays. Fortunately, one can design an equivalent system which presents a much larger solid angle of acceptance. In Appendix $C$, the preceding discussion is extended to include some more effictent arrangements. In section II B below, the same problem is congldered from a more abstract and general point of vlew.

Beyond the design of more efficlent version of the present gystem, it is also of interest to consider other possfible observa- 
tions. Selection of a single vivelength camponent of the distribution of sources within a local volume is not the or:iy type of spatial resolution which could $k$ achieved. Spectroscopic systems can be designed to use phase information for a variety of purposes. Thts possibil:ty greatly extends the scope of the problem. It is important not only to consider a variety of optial systems, but also to descsibe in general terms the range of possible measurements. The necessary analysis is not completed in this study, kut in the rext few sections of the discussion, several ways of looking at the prolem are considered.

Kany plasma phenomena occur at frequencies toc high for direct time measurements. The low frequency assurption of the preceding analysis is thus a severe restriction on the utility of the suggested method. Fortunately, it does not appear to be a necessary IImitation. Some modifications of the apparatus which would permit observation of higher frequency phenomena are proposed in Chapter V.

The low-frequency limit is nevertheless a convenient initial stmplification. It essentially permits one to consider first the spatial or $\underline{k}$ dependence of the problem and to defer discussion of the time or frequency dependence. Both the anelysis presented in this chapter and the observations described in Chapter $I V$ pertain to this one aspect of the problem. 3. A Reformulation of the Two-Beam Problam

In the preceding discussion of a two-beam spectrometer, severel assumptions are stated or implied: 
1. The effect of the plasma is represented by a scalar source Jensity, $s(\underline{r}, t)$, which is not affected by other elements of the optical problem.

2. One IInearly polarized component of the emitted light is accepted by the opt1cal system. It 1s assumed that the amplitude $\xi(\underline{r}, t)$ of this radiation is related to the source by a scalar vave equation Eq. (I.3),

$$
\nabla_{\xi}(\underline{r}, t)-\frac{1}{c^{2}} \frac{\partial^{2} \xi(\underline{r}, t)}{\partial t^{2}}=-4 \pi s(\underline{r}, t)
$$

whlch has the retarded solution ${ }^{38}$

$$
\xi(\underline{r}, t)=\int a^{3} r^{\prime} \frac{1}{\left|\underline{r}-\underline{r}^{\prime}\right|} s\left(\underline{r}^{\prime}, t-\frac{\left|\underline{\underline{r}}-\underline{\underline{r}}^{\prime}\right|}{c}\right) .
$$

3. The plaswa is observed through two beems which are reatricted as to regions of sbservation, directions $\left(\hat{k}_{A}, \hat{k}_{B}\right)$ of emission of accepted light, and opt1cal wevelength $\left(2 \pi /\left|\underline{k}_{A}\right|+2 \pi / \underline{k}_{B} \mid\right)$. The accepted light is conveniently described in terms of a spatial Fourler transform of the wave amplitude

$$
\begin{aligned}
& \xi_{A, B}\left(\underline{k}_{A, B}, t\right) \equiv \int_{(a l 1} d^{3} r \xi_{A, B}(\underline{r}, t) e^{-i k k_{A, B} \cdot \underline{r}} \\
& \text { spece) } \\
& =\int_{(\text {all }} a^{3} r \int_{\substack{\text { (region } \\
\text { observed) }}} a^{3} r^{+} \frac{1}{\left|\underline{r}-\underline{r}^{\prime}\right|} \\
& \theta\left(\underline{r}^{\prime}, t-\frac{\left|\underline{r}-\underline{r}^{\prime}\right|}{c}\right) e^{-1 \underline{k} A, B^{*} \underline{r}}
\end{aligned}
$$




$$
=\int_{\substack{\text { (all } \\ \text { space) }}} d^{3} r^{\prime} \xi_{A, B}\left(\underline{k}_{A, B^{\prime}}, t ; \underline{r}^{\prime}\right),
$$

where

$\xi_{A, B}\left(\underline{k}, t ; \underline{r}^{\prime}\right) \equiv\left\{\begin{array}{l}\left\{d^{3} r \frac{e^{-1 \underline{k} \cdot \underline{r}}}{\left|\underline{r}-\underline{r}^{\prime}\right|} s\left(\underline{r}^{\prime}, t-\frac{\mid \underline{r}-\underline{\underline{r}^{\prime} \mid}}{c}\right)\right. \\ \text { for points } \underline{r}^{\prime} \text { within } \\ \text { the observed beam and } \\ 0 \text { for all other points } \underline{\underline{r}}^{\prime}\end{array}\right.$

is the $\underline{k}$ component of the radiation from sources at points $\underline{r}^{\prime}$ observed through beem $A$ or $B$.

Since the negative frequency components of $\xi_{A, B}\left(k_{A}, B, t\right)$ propagate in the $-\hat{k}_{A, B}$ direction, and since the source is observed from the $+\hat{k}_{A, B}$ directions, only the positive frequency components, $\xi_{A, B}^{(+)}\left(k_{A, B}, t\right)$, are recelved by the detector. (This point is discussed in detail in Appendix E. 1.) The omplitudes of the transmitted waves, which are, of course, real valued quantities, may be expressed in terms of $\xi_{A, B}^{(+)}\left(k_{A}, B, t\right)$. See $E q .(E . I 7)$ and $d i s-$ cussion.) To within a multiplicative constant

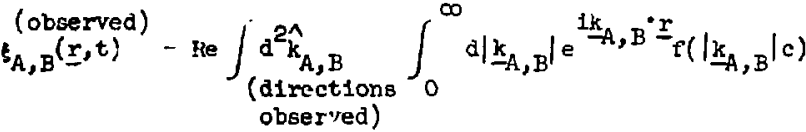

$$
\begin{aligned}
& \text { - } \frac{\left|k_{A_{2} B}\right|}{2 \pi 1 c} \xi_{A, B}^{(r)}\left(k_{A, B}, t\right)
\end{aligned}
$$

Where $f\left(\left.\right|_{A, B} \mid c\right)$ is the transfer function of the spectral filter. The light in each beam may then be expressed as the superposition 
of contributions from different points within the plasma. Combining Eqs. (II.2) and (II.3), we have

$$
\underset{\xi_{A, B}(\underline{r} \cdot t)}{\text { (observed) }}=\int a^{3} r^{\prime} \quad \begin{aligned}
& \text { (observed) } \\
& \xi_{A, B}\left(\underline{r}, t ; \underline{r}^{\prime}\right)
\end{aligned}
$$

where

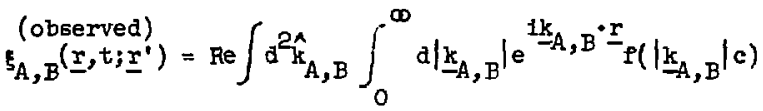

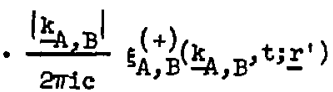

4. The light in beams $A$ and $B$ is then combined and reseparated into two complementary "Interference patterns," beams 1 and 2, whose time averaged intensities are measured. The optical system which combines the two beams serves to superimpose the light from different points: $\underline{r}_{A}$ within beam $A$ and $\underline{r}_{B}$ within beam B.

$$
\begin{aligned}
& I_{1,2}(t)=\underbrace{}_{\frac{1}{\sqrt{2}}-\left.\left[\begin{array}{l}
\text { (observed) } \\
\xi_{B}\left(\underline{r}_{B}, t\right)
\end{array} \xi_{\xi_{A}\left(\underline{r}_{A}, t\right)}^{\text {(observed) })}\right]\right|^{2}}
\end{aligned}
$$

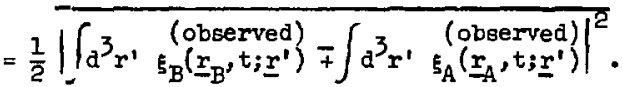

5. Light emitted from different points within the plasma is completely incoherent. Each measured intensity is just the sum of Intensities of light from different points. Thus Eq. (II.5) becomes 


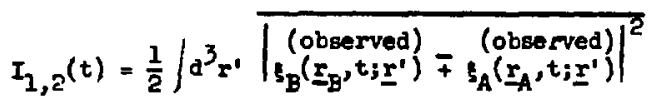

Since beams $A$ and $B$ are transmitted through the seme spectrel fllter, $f\left(\left|k_{A}\right| c\right)=f\left(\left|k_{B}\right| c\right)$ and Eqs. (II.4) and (II.6) reduce to

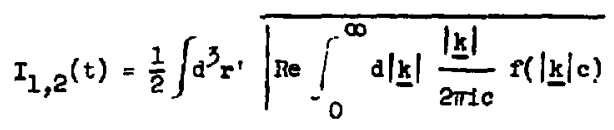

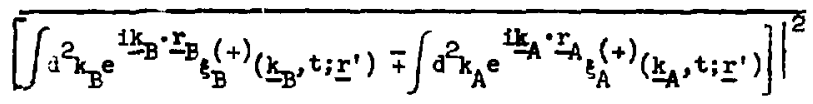

6. Beams A and B are narrow bundles of rays, each s'tbtending a small solid angle $\delta^{2} \Omega$. The amplitude $s_{A, B}$ does not rary over the width of either beam, so the integrations over direction $d^{2} k_{A, B}$ merely introduce a factor of $5^{2} \Omega$.

7. The integration time over which the intensities are averaged exceeds the coherence time of the light, so the messured intensities are just the sumb of the intensities of different spectral components,

$$
\begin{aligned}
& I_{1,2}(t)=\frac{1}{2} \int d^{3} r^{\prime} \int_{0}^{\infty} d|\underline{\underline{k}}|\left(\frac{|\underline{\underline{k}}|}{2 \pi c}\right)^{2}\left(\delta^{2} \Omega\right)^{2}
\end{aligned}
$$

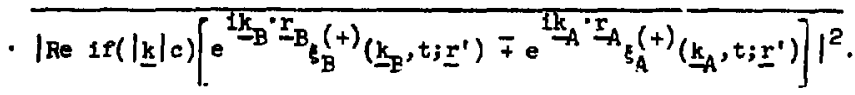


Intonsties can be exprossed in terms of the assocleted analytic elonal. [see Eq. (E.15).] it is conventent to make this subst1tulon here. Doing so, and using the result obtain in Append1x E.2 leads, after a few algebraic steps, to the expression

$$
I_{1,2}(t)=\int d^{3} r^{\prime} \int_{0}^{\infty} d|\underline{k}||f(|\underline{k}| c)|^{2} I_{1,2}\left(t,|\underline{k}| ; \underline{\underline{r}}^{\prime}\right)
$$

where

$$
\begin{aligned}
& I_{1,2}\left(t,|\underline{k}| ; \underline{r}^{\prime}\right)=\frac{1}{4}\left(\frac{|\underline{k}| 8^{2} g}{2 \pi c}\right)^{2}
\end{aligned}
$$

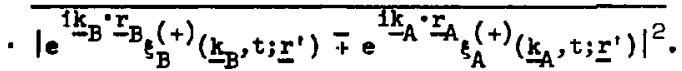

8. Each location within the placue is an 1sotropic source of light whose coherence length exceeds any differences in opt 1cal path through the oystem. The contributions to beams A and B fram the same source point are completely coherent. The1r combined effect is found by adding the two component amplitudes before time everaging.

The recorded output of the instrument is the difference botween the two measured intensities,

$$
\begin{aligned}
Y(t) & =I_{2}(t)-I_{1}(t) \\
& =\int d^{3} r^{\prime} \int_{0}^{\infty} d|\underline{k}||f(\underline{\underline{k}} \mid c)|^{2}\left[I_{2}\left(t,|\underline{k}| ; \underline{r}^{\prime}\right)-I_{1}\left(t,|\underline{k}| ; \underline{r}^{\prime}\right)\right] .
\end{aligned}
$$

Using Eq. (II.9b) givea, after a few algutratc steps, 


$$
\begin{aligned}
& I_{2}\left(t,|\underline{\mathbf{k}}| ; \underline{\mathbf{r}}^{\prime}\right)-I_{1}\left(t,|\underline{\mathbf{k}}| ; \underline{\underline{r}}^{\prime}\right)=\left(\frac{|\underline{\mathbf{k}}| \delta^{2} \Omega}{2 \pi c}\right)^{2} \\
& \text { - }\left\{\operatorname{Re} \sqrt{\left.e^{-1 \underline{k}_{A} \cdot \underline{r}_{A_{\xi}}(+)^{*}}\left(\underline{k}_{A}, t ; \underline{r}^{\prime}\right) e^{1 \underline{k_{B} \cdot \underline{r}_{B_{E}}(+)}\left(\underline{k}_{B^{\prime}}, t ; \underline{r}^{\prime}\right)}\right\}}\right. \\
& =\operatorname{Re} \Gamma_{\mathrm{BA}}\left(0 ;|\underline{\mathbf{k}}|, \underline{\mathbf{r}}^{\prime}\right)
\end{aligned}
$$

where the correlation $\Gamma_{\mathrm{BA}}\left(\tau ;|\underline{\mathbf{k}}|, \underline{\mathbf{r}}^{\prime}\right)$ is defined

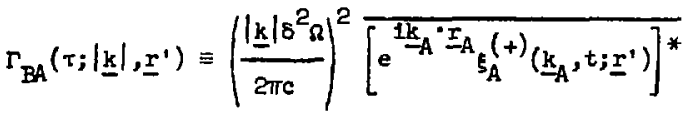

$$
\begin{aligned}
& \text { - } \overline{\left.e^{\underline{I k} \cdot \underline{r}_{B_{s}}(t)}\left(\underline{k}, t+\tau, \underline{r}^{\prime}\right)\right]} \text {. }
\end{aligned}
$$

Thus the observed signal is just an integral of correlations

$$
\mathbf{Y}(t)=\int_{\substack{\text { (common } \\ \text { source volume) }}}^{a^{3} \mathbf{r}^{\prime}} \int_{0}^{\infty} \mathrm{d}|\underline{k}||\mathbf{f}(|\underline{\mathbf{k}}| \mathrm{c})|^{2} \operatorname{Re} \Gamma_{\mathrm{EA}}\left(0 ;|\underline{k}|, \underline{\mathbf{r}}^{\prime}\right)
$$

between the $\underline{k}_{A}$ and $\underline{k}_{B}$ components of the light. Since $\Gamma_{B A}$ is clearly zero if either factor vanishes, the $\underline{r}^{\prime}$ Integration mey be restricted to the common source volume as expected.

Equations (II.2b) can now be used to express the correlatjon $\Gamma_{B A}\left(0 ;|\underline{k}|, \underline{r}^{\prime}\right)$ in terms of the given source distribution, $s\left(\underline{r}^{\prime}, t\right):$ 
$-52-$

$$
\begin{aligned}
& \Gamma_{B A}\left(0 ;|\underline{k}|, r^{\prime}\right)=\left\langle\frac{\left.|\underline{k}| 8^{2} \mathfrak{q}\right|^{2}}{2 \pi c}\right\rangle^{-i \underline{k} \cdot e_{A} \cdot \underline{r}_{A}} e^{+1 \underline{k}_{B} \cdot \underline{r}_{B}} \\
& \cdot d^{3} r_{1} \frac{e^{1 \underline{k}_{A} \underline{r}_{1}}}{\left|\underline{r}_{1}-\underline{r}^{\prime}\right|} s^{(+)^{*}}\left(\underline{r}^{\prime}, t-\frac{\left|\underline{r}_{1}-\underline{r}^{\prime}\right|}{e}\right) \\
& \cdot \int d^{3} r_{2} \frac{e^{-1) \underline{r}_{B} \cdot \underline{r}_{2}}}{\left|\underline{r}_{2}-\underline{r}^{\prime}\right|} \theta^{(+)}\left(\underline{r}^{\prime}, t-\frac{\left|\underline{r}_{2}-\underline{r}^{\prime}\right|}{c}\right) \\
& =\left(\frac{|\underline{k}| \delta^{2} \Omega}{2 \pi c}\right)^{2} e^{-i \underline{k}_{A} \cdot \underline{x}_{A}} e^{+1 \underline{k}_{-} \cdot \underline{x}_{B}} e^{1 \underline{k} \cdot{ }^{\prime} \underline{x}^{\prime}} e^{-i \underline{k} \cdot \underline{x}^{\prime}}
\end{aligned}
$$

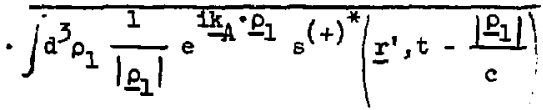

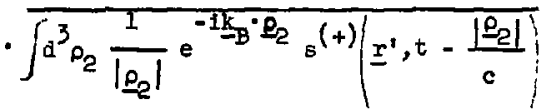

(II.14)

where $\quad \underline{\rho}_{1} \equiv \underline{x}_{1}-\underline{x}^{\prime}$

and $\quad \underline{\rho}_{2} \equiv \underline{r}_{2}-\underline{r}^{\prime}$.

This expression reduces to (see Appendix B.2 for details),

$\Gamma_{B A}\left(0 ;|\underline{k}|, \underline{r}^{\prime}\right) \underset{t \rightarrow \infty}{\rightarrow} e^{-\underline{1} \underline{k}_{A} \cdot\left(\underline{r}_{A}-\underline{r}^{\prime}\right)} e^{1 k_{B} \cdot\left(\underline{r}_{B}-\underline{r}^{\prime}\right)}\left(\delta_{\Omega}^{2}\right)^{2} A^{\prime}\left(|\underline{k}| c ; \underline{\underline{r}}^{\prime}\right)$

(II.15a)

where

$$
\delta\left(|\underline{\underline{k}}| c ; \underline{\underline{r}}^{\prime}\right) \equiv\left|\mathrm{s}^{(+)}\left(\underline{\underline{r}} \underline{ }^{\prime},|\underline{k}| c\right)\right|^{2}
$$


is the spectrum of the light emitted from $\underline{r}^{\prime} \cdot\left[{ }_{s}^{(+)}\left(\underline{r}^{\prime},|\underline{k}| c\right)\right.$ is the temporal Fourler transform of $s^{(+)}\left(\underline{r}^{\prime}, t\right), i . e .$, the positive frequency portion of the transform of $s\left(\underline{r}^{\prime}, t\right)$. The spectrum $\mathcal{L}\left(|\underline{k}| c ; \underline{r}^{\prime}\right)$ is thus defined over positive optical erequencles $|\underline{x}| \mathrm{c}$.

The time average in Eq. (II.15b) is actually superfluous, since the averaged quantity is constant. This is a consequence of the introduction of the analytic signal in Eq.(II.9); if the real field anplitude had been retalned, an average would be needed to define a constant Iight intensity.

More important, however, is the fact thet a real time average is always taken over a finite interval. In a real system, If $\Delta$, the spectrometer bandwidth, is relatively nerrow, the output light is nearly monochromatic. Then an average of the Intens ${ }^{\prime}$ ty over a time $T \sim N^{-1}$ eliminates the optical frequency variation but retains a slow time dependence in the measured spectrm. The result of this operation (which is considered more carefully in Appendix E.j) is a time varying algnal, not a aingle long time linit as above. To describe this result, Eq. (II.15) can simply be rewritten in the form,

$\Gamma_{B A}\left(0 ;|\underline{k}|, \underline{r}^{\prime}, t\right)=e^{-1 k_{A} \cdot\left(\underline{r}_{A}-\underline{r}^{\prime}\right)} e^{+1 k_{B} \cdot\left(\underline{r}_{B}-\underline{r}^{\prime}\right)}\left(\delta^{2} \Omega\right)^{E},\left(1|\underline{\underline{k}}| c ; \underline{r}^{\prime}, t\right)$

Since the spectral amplitude is real, the phase of the correlation is determined iy the factor,

$$
e^{i\left[\underline{k}_{B} \cdot\left(\underline{r}_{B}-\underline{r}^{\prime}\right)-\underline{k}_{A} \cdot\left(\underline{r}_{A}-\underline{r}^{\prime}\right)\right]} .
$$


The two termg in the exponent are just the phase differences along the paths from the oource point $\underline{r}^{\prime}$ to the two observation points. The phase of the correlation, that 1s, the relative phese of the components of the light observed through the two beans, is juet determined by the difference between the lengthe of the two optical paths.

In general, this difference would depend both upon $\underline{r}^{\prime}$ and upon $|\underline{k}|$. However, 1t was assumed above that the light from each point $\underline{r}^{\prime}$ is coherent, that 1 ts coherence length exceeds the differences in opt1cal path. For a thermal plasma, the cohprence length 1s just determined by the width of the spectrum of the transmitted light. The requirement of coherence simply means that the light is so nearly monochromatic and the path differeences are so small that the phese difference is the same for all components of the spectrum. Under this assumption, the phase of the correlation depends upon $\underline{\underline{x}}^{\prime}$, but not explicitly upon $|\underline{\mathbf{k}}|$. The expression (II.17) may thus be written

$$
e^{10} e^{-1 k} \Delta^{\prime} \underline{r}^{\prime}
$$

where

$$
\emptyset \equiv \underline{k}_{B} \cdot \underline{r}_{B}-\underline{k}_{A} \cdot \underline{r}_{A}
$$

and

$$
\underline{k}_{\Delta} \equiv \underline{k}_{B}-\underline{k}_{A}
$$

may be defined for one typical optical wavelength and then treated as constants, indepandent of $|\underline{k}|$. Equations (II.13) and (II.16) then give the final forw, 


$$
\begin{aligned}
& Y(t)=\left(s^{2} a\right)^{2} \int_{0}^{\infty} d|\underline{k}||t(|\underline{k}| c)|^{2} \\
& \text { - } \operatorname{Re}\left[e^{1 \phi} \int_{\substack{\text { cormon } \\
\text { source volume }}} a^{3} r^{\prime} e^{-i \underline{k} \Delta^{\prime} \underline{r}^{\prime}} \Delta\left(|\underline{k}| c ; \underline{r}^{\prime}, t\right)\right] \\
& =\left(\delta^{2} a\right)^{2} \operatorname{Re}\left[e^{1 \phi} \int_{0}^{\infty} d|\underline{k}||f(|\underline{k}| c)|^{2} \underset{\substack{\text { common } \\
\text { sources }}}{2(|\underline{\underline{k}}| c ; \underline{\underline{k}}, t)]}\right.
\end{aligned}
$$

So, to within a phase factor, the result depends only upon $\alpha\left(|\underline{k}| c ; \underline{k}_{\Delta}, t\right)$, the $\underline{\underline{k}}_{\Delta}$ spatial Fourler component of the districomon sources

bution of those sources of light of frequency $|\underline{k}| c$ which are observed through both beams, and upon $|f(|\underline{k}| c)|^{2}$, the transmission function of the spectral filter. The time dependence of the output simply follows the time dependence of tht observed component of the light source distribution.

only one phase of the complex valued $\mathcal{L}\left(|\underline{k}|<; k_{0}, t\right)$ is common sources

bere observed but, as explained in the next section, the optical system could easily be modffied to provide both the real and the imaginary parte of $e^{16} \mathrm{~S}$.

It should be exphasized that the assumpt Ion of coherence doos not Imply that the interference must pe the same for all eccopted wavelengtbs. Sources or' different portions of a spectral line, for example, wight vell be differently distributed in space. Different portions of a stark broadened 11ne, emitted from regions 
of different electric field, or different portions of a Doppler broadened line, emitted by sources moving with different velocft1os could exhibit different dependence upon $k_{\Delta}$ and $t$, even though the spectrum was quite narrow. The assumption of coherence simply means that the relation between the location of the source and the resulting interference pattern is the same for all accepted vavelengths. So long as this is true, the measured distribution vill be just the sum of the distributions of all the sources which are observed in any single measurement.

If the spectrum of accepted light were so wide that the light was not coherent, the total signal would be due to different components of the distributions of sources of light of different wavelengths. This is not to say that such a measurement could not be usefur, but only that it is not covered by the foregoing analysis. Some ways in whicis a larger partion of the spectrum might be used are considered in chapters $V$ and VI below.

For a two-beam epectroscoplc observation, made with a narrow portion of the spectrum, Eq. (II.19) confirms the conclusions of our firat ansiygie. The output, $Y(t), g$ lves a messure of $\mathcal{S}\left(|\underline{k}| c ; \underline{k}_{\Delta}, t\right)$, the $\underline{k}_{\Delta}$ component of the common source distribution. common sources

If desired, a spectrum analyzer could be used to messure the (low) eroquency spectrum $Y(\omega)$ of the output, which would give $\boldsymbol{S}(|\underline{k}| c ; \underline{k}, \omega)$, the complete Fourler trensform of the distribucomoon sources

tion of light sources within the common source volume. According to the Wleser-Khintchine theorem, a complete 
cessurament of $\dot{j}(|\underline{\underline{k}}| c ; \underline{k}, \omega)$ vould provide the two-point, two. common sources

time correlation function of the source distribution.

As in scattering measurement (see section J.cC), only one $\underline{k}$ component, the $\underline{k}_{\Delta}$ component of the source density fluctuations would be okserved at once, but with a two-beam spectrometer, one could examine a varlety of plaswa wavelengtins by varylng elther the angle between the two beams or the opt1cal wavelength accepted. In an arrangement like that of Fig. II-2, the angle between beams A. and B could be changed by replacing the first lens by another of different focal length. The wavelength could be changed by changing the spectral f1lter, but of course it should be remembered that different optical wavelengths may be due to different types of source. As in any sfectroscopic study, the relation between components of the spectrum and conditions in the plasme 18 a complicated matter which requires a separate analysis. With a conventional spectrometer, only the total intensity of each component of the spectrum 1s observed. W1th a two-beam spectrometer, one could, in principle, abserve the two-point, two-time currelation function of the distribution of the sources of any feature in the spectrum of the light emitted by a plasme.

\section{B. Multiple-Beam Systems}

\section{The Use of Polartzation}

The foregoing analysis was based upon a scalar wave equatyon, or, more preclsely, upon a retarded Green's function solution to such an equation. Th1s common, useful procedure is 
easily interpreted: The scalar equation describes a single polarization component of the fleld. Such a description applies to meny apectroscople measurements, in which a single lineariy or circularly polarized component of the light is used. Since, in many altuations, light waves of alfferent polarization remain distinct, a sialar analysis is often justified. However, even If the source is considered to be a scalar, and even if a scalar wave equation is used to celculate the amplitude of the emftted light, the fact that light is actually a vector wave 1s still important in the present problem.

The vector nature of the wave should be considered, first of all because the palarizability of light provides a most convenient way to measure phase relations. This fact is the besis of a class of instruments called polarization interferometers whlch are described in a recent book. ${ }^{39}$ A polarization interferometer is a device in which two beams of light are differently polarized and then combined. The polarization of the resulting light depends upon the relative phase, as well as the polarization of the original component weves. Because of this dependence, a measurement of the resulting polarization gives information about the pheses of the component waves, the same information which, in a conventional Interferometer, is provided by a study of a jattern of interference fringes.

In most polarization interferometers, the interfering waves (waves $A$ and $B$ of the preceding discusston) are linearly polarIzed in orthogonal directions, as shown in F1g. II-5. If two 


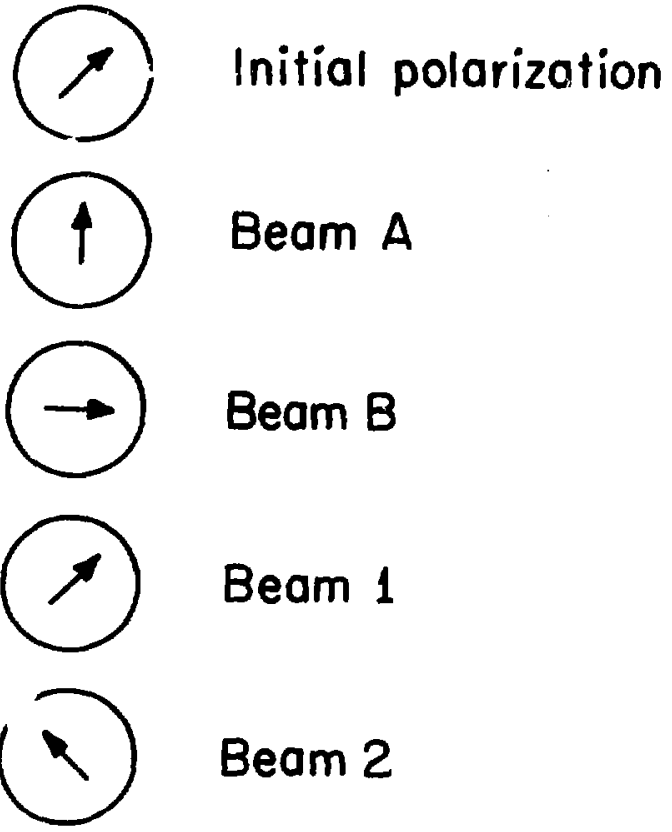

XBL733-2405

Fig. II-5. Polarization of the different beams. 
such waves are equal in amplitude, and also equal in phase, their superposttion will again be linearly polarlzed, in the direction "I" shown in Fig. II-5. If the interfering waves are 180 deg out of phase, the1r superposition will be linearly polar1zed in the orthogonal direction "2". These two polarization components are exactly equivalent to the "Interference patterns 1 and 2 " which were considered in detail in Section II.A above.

Th1s equivalence provides a very conventent way to actually construct a two-beam spectroscopic system. Several possible dealgns for such an instrment are described in detail in Appendix C of this diseussion. Essentialiy, ode must use un initial polerfzer to select one component of the emitted light, then polarize the obgerved beams $A$ and $B$ as shown in FIg. II-5, combine them, and se rate the result into poleriza، in components 1 and 2 , which are otserved with separate phototubes. Then, if beams A and I are coherent, their superposition will be polerized, and the light will be divided between phototubes 1 and 2 in a manner detemined by the relative phase of $A$ and $B$, exactly as described by the smalysis of section II.A.3. If the light in beams $A$ and $B$ 18 incoherent, their superposition will be unpolerized, the light will be divided equally between the two phototubes, and the output olgnal, $Y(t)=I_{2}(t)-I_{1}(t)$ w1ll be zero. If the two observed beams are partially coherent, thelr ouperposition will be partially polarized, and, fust as before, only the coberent portion of the 11ght will contribute to the output of the system.

Thore are severel advantages to this technlque. A sorting out of different sets of interference fringes might be difficult, 
but the orthogonally polarized components "l" and "2" can easily be separated. If beams $A$ and $B$ are distingulshed by their polarization, they can be superimposed and portions of their paths maie physically identical, insuring equality of path length and minfmizing the effect of vibrations and misaligments in the system. Such a procedure also reduces the number of optical components needed, since, roughly speaking, svery element then counts as two. Several other reasuns ior using polarization are examined later, in Appendix c.

One potential advantage, which we shall not consider further, but which should at least be mentioned, 1s the possibility of measuring the complete complex mutual coherence of the two observed light beams. Recall. that the two-beam system analyzed above wis shown to measure an Integral over sources and Prequencles of

$$
\operatorname{Re}\left[e^{1 \phi} \Gamma_{B A}\left(0 ;|\underline{k}|, \underline{r}^{\prime}\right)\right]
$$

[see Eq. (II.13], where $\Gamma_{B A}$ is the mutual coherence and $e^{1 \phi}$ is a constent factor. The restriction to the real part of the expression is a consequence of the way in which the interference was observed. The system separated "Interference patterns 1 and 2," with pattern I produced if beams $A$ and $B$ were--apart from the phese difierence $\phi$--equal in phese and pattern 2 produced if they wers $180 \mathrm{deg}$ out of phase. However, 1f A and B were 90 deg apart In phase, the fringe pattern would be exactly intermediate between 1 and 2, the ligat would be divided equally between the two photo- 
tubes, and a null output would result--exactly as if the light weit incoherent. Such unobserved correlations are represented by the missing part of the mutual coherence,

$$
\operatorname{Im}\left[e^{1 \phi} \Gamma_{B A}\left(0 ;|\underline{k}|, \underline{r}^{\prime}\right)\right] \text {. }
$$

In a polarization interferometer, such a correlation would make the output circularly polarized. Since this effect could also be observed, both the real and the imaginary parts of the mutual coherence could, in princtple, be measured, a possibility expiained in more detall in Ref. 39.

Aftc $r$ discussing a two-beam spectrometer, it is natural to imagine extending the method by designing a system to compare light emitted in many directions from a plasma. Such a multiplebeam spectrometer would certainly be more efficient than a twobeam system, since more light could be used, and furthermore, the more complex arrangement should make possible a great variety of spectroscoplc messurements.

Thus one could proceed now to consider in succession threeand four- and five-beam spectroscoplc gystems. However, when the use of polarization is Included, the simple two-beam system suggeste a different kind of generalization. Since orthogonally polarized beems may be superimposed w1thout loss of ident1ty, one can, with a polarizing system, define not Just more beams, but two whole sote of beems, "beame A" and beams B," polarized as A and $B$ in Fls. II-5.

Within the optical system, the $A$ and $B$ components cannot only 
be recognized, they can also be independently mantpulated. If optical components made of birefringent materlals are included In the system, the optics seen by the $A$ and $B$ components of the light can be completely different. This possibility, which is really the most important reason for using polarizing optics, leads us to replace the simple two-beam system by a much more general type of apparatus. In place of the two apertures which defined beams $A$ and $B$, there can be two whole optical systems, $A$ and $B$, followed by a polarization interferometer to measure the correlation between the two resulting waves.

2. Mult: pie-Beam Systems in the Hurgens Approximation

A general spectroscopic system of this type is shown in F18. II-6. There a lens, with focal point within the plasma, Is followed by a linear polarizer, which insures that the system operetes with only one component of the 11ght. The amplitude of th1s transmitted wave, $\xi_{0}(\underline{x}, t)$, may be treated as a scalar and releted to a given ecalar source by expression

$$
\begin{aligned}
\xi_{0}\left(\underline{r}^{(1 n)}, t\right)=\int d^{3} r^{\prime} \frac{I}{\left|\underline{r}^{(1 n)}-\underline{r}^{\prime}\right|} \\
\\
\cdot s\left(\underline{r}^{\prime}, t-\frac{\left.\left|\underline{r}^{(1 n)}-\underline{r}^{\prime}\right|+\phi_{I} \underline{\underline{r}}^{(1 n)}\right)}{c}\right)
\end{aligned}
$$

where $\underline{r}^{\prime}=\left(x^{\prime}, y^{\prime}, z^{\prime}\right)$ refers to points within the plaswa and $\underline{x}^{(1 n)}=\left(x^{(1 n)}, y^{(1 n)}, z^{(1 n)}\right)$ denotes points Imediately behind the lons. $\left(x^{\prime}=x^{(1 n)}=y^{\prime}=y^{(1 n)}=0\right.$ along the axis of the syotom.) 


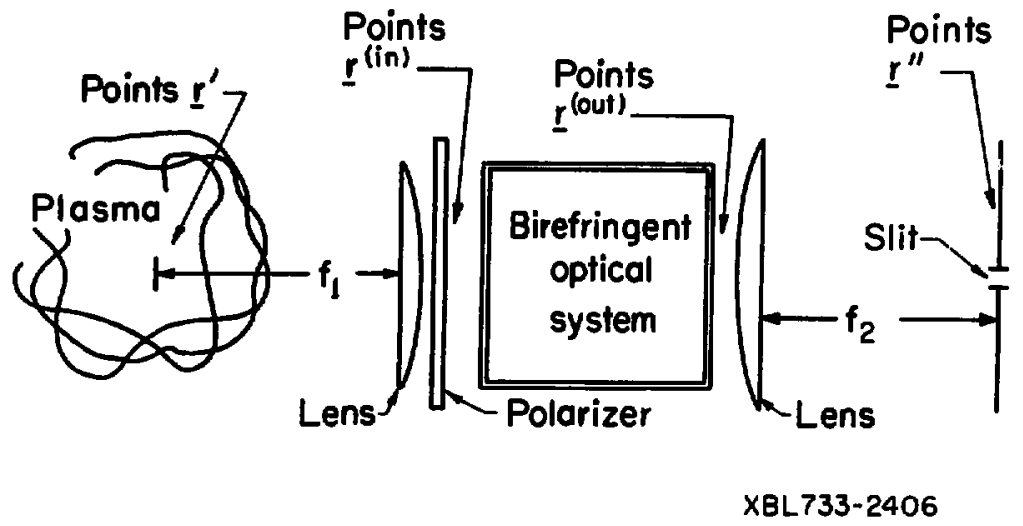

F1g. II-6. A multiple-beam spectroscoplc system. (The slit shown at the right is the entrance to a spectrometer.) 


$$
\left.\phi_{1} \underline{\underline{r}}^{(1 n)}\right)=\phi_{10}-\frac{1}{2} \frac{x^{(1 n)^{2}}+y^{(1 n)^{2}}}{f_{1}}
$$

1s the additional path introduced by the lens, which hes a focal length of $\mathbf{f}_{1}$.

This wave, $\xi_{O}$, 1s then considered to consist of $A$ snd $B$ components, which are inftially 1dentical, except in polarization. The first lens is followed by an optical system which affects the A and B components differently. That is, the system contains birefringent elements with axes orlented so that the $A$ and $B$ components remein distinct and independent, but follow different piths. Assuming linearity and time-independence of the system, the effect of such an apperatus is described by two Green's functions, $g_{A}$ (r) $\left.^{\text {(out) }}, \underline{r}^{(\text {In })}, \tau\right)$ and $g_{B}$ (r) $\left.^{\text {(out })}, \underline{\underline{r}}^{(1 n)}, \tau\right)$ : $\left.\xi_{A, B} \underline{r}^{(\text {out })}, t\right)=\int a^{3} r^{(1 n)} \int_{0}^{\infty} d \tau$

$$
\text { - } \left.\left.B_{A, B} \underline{x}^{(\text {out })}, x^{(\ln )}, \tau\right) \xi_{O} \underline{x}^{(\ln )}, t-\tau\right) \text {. }
$$

Here $\underline{r}^{\text {(out) }}=\left(x^{\text {(out) }}, y^{\text {(out) }}, z^{\text {(out) })}\right.$ denotes points across the output of the system.

The birefringent portion of the syatem is followed by a second lens of focal length $f_{2}$ which focuses parellel light onto a plane $\left[\right.$ of nolnts $\left.\underline{x}^{\prime \prime}=\left(x^{\prime \prime}, y^{\prime \prime}, z^{\prime \prime}\right)\right]$ conteining the entrance to a epectrameter. The light at points $\underline{\underline{x}}^{\prime \prime}$ is related to the light before the cecond leng by two KIrchoff Integral expressions: 40 


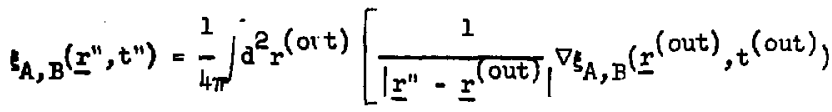

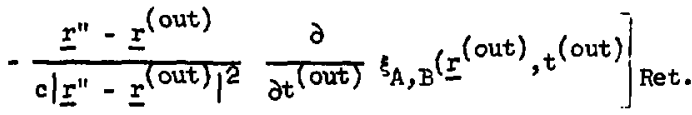

Ret.: $t^{\text {(out) }}=t^{\prime \prime}-\frac{\left|\underline{x}^{\prime \prime}-\underline{r}^{\text {(out })}\right|+\phi_{2}\left(\underline{\underline{r}}^{\text {(out) })}\right.}{c}$.

Here

$$
\phi_{2} \underline{\underline{x}}^{\text {(out) })}=\phi_{20}-\frac{1}{2} \frac{x^{\text {(out })^{2}}+y^{\text {(out })^{2}}}{f_{2}}
$$

1s the added path length produced by the lens. $\left[x^{\text {(out })}=x^{\prime \prime}=\right.$ $y^{\text {(out) }}=y^{\prime \prime}=0$ along the axis of the system.] Here it is assumed that the normal component of $\nabla \xi(\underline{r}, t)$ just after the lens is approximately given by

$$
\nabla \xi\left[\underline{r}^{\text {(out) }}, t-\frac{\left.\phi_{2} \underline{\underline{r}}^{\text {(out })}\right)}{c}\right] \cdot d^{2}{ }^{\text {(out })} .
$$

A term of higher order in $\left[\right.$ (wavelength)/| $\left.\underline{\underline{r}}^{\prime \prime}-\underline{r}^{\text {(out) }} \mid\right]$ has also been neglected. In this expression one can make the usual Huygens approximation by assuming that $\xi=0$ bekind any makks and is unperturbed at the points across an eperture.

Altogether, Eqs. (II.20), (II.21), and (II.22) determine $\xi_{A}\left(\underline{r}^{\prime \prime}, t^{\prime \prime}\right)$ and $\xi_{B}\left(\underline{r}^{\prime \prime}, t^{\prime \prime}\right)$ in terms of $s\left(\underline{r}^{\prime}, t^{\prime}\right)$. Since the differout steps are linear relations, their ccmbined effect is also Linear. Hence if $\xi_{A}, \xi_{B}$, and $s$ ere Fourler trensformed in time, 
the flelds at any point $\underline{x}^{\prime \prime}$ produced by a source at a point $\underline{r}^{\prime}$ w11l be determined by two complex-valued transfer functions, $\emptyset_{A}\left(\underline{r}^{\prime \prime}, \underline{\underline{r}}^{\prime},(1)\right)$ and $\emptyset_{B}\left(\underline{r}^{\prime \prime}, \underline{r}^{\prime}, \infty\right)$.

$$
\xi_{A, B}\left(\underline{\underline{x}}^{\prime \prime}, \omega ; \underline{\underline{r}}^{\prime}\right)=\emptyset_{A, B}\left(\underline{\underline{r}}^{\prime \prime}, \underline{\underline{r}}^{\prime}, \omega\right) B\left(\underline{\boldsymbol{r}}^{\prime}, \omega\right)
$$

W1thin the spectrometer, however, it is not the $A$ and $B$ components, but rather the Intermediate polarizations "I" and "2" wh1ch are separated.

$$
\begin{aligned}
& \xi_{1}=1 / \sqrt{2}\left(\xi_{B}-s_{A}\right) \\
& \xi_{2}=1 / \sqrt{2}\left(\xi_{B}+\xi_{A}\right) .
\end{aligned}
$$

Since all of the light of the proper frequency which enters the spectrometer is reflected into one of the phototubes, each measured intensity is given by an integral over the spectrometer entrance aperture. Assuming that the light is nearly normal to this surface,

$$
\begin{aligned}
I_{1,2}\left(t^{\prime} ; \underline{r}^{\prime}\right)= & \int_{\begin{array}{c}
\text { spectrameter } \\
\text { entrance })
\end{array}}^{d^{2} r^{\prime \prime}} \int_{0}^{\infty} \frac{d \omega}{2 \pi}|f(\omega)|^{2} . \\
& \cdot\left|s_{B}\left(\underline{I}^{\prime \prime}, \infty ; \underline{r}^{\prime}, t\right) \mp s_{A}\left(\underline{r}^{\prime \prime}, \omega ; \underline{r}^{\prime}, t\right)\right|^{2} .
\end{aligned}
$$

Here, as in the analysis in section II.A.3, $|f(\omega)|^{2}$ is the spectrameter transmission function. Also as before, a slow time dependence has been included here in the measured spectra. Since the plasma is an incoherent source, each intensity is juat a sum of contributions fram the different points $\underline{r}^{\prime}$. 


$$
I_{1,2}(t)=\int \mathrm{d}^{3} r^{\prime} I_{1,2}\left(t ; I^{\prime}\right)
$$

The output of the gystem 18 the difference signel

$$
\begin{gathered}
Y(t)=I_{2}(t)-I_{1}(t)=\int d^{3} r^{\prime} \int_{0}^{\infty} \frac{d w}{\pi}|f(\omega)|^{2} \int_{\substack{\text { (spectrometer } \\
\text { entrance) }}}^{d^{2} \mathbf{r}^{\prime \prime} 2} \\
\cdot \operatorname{Re}\left[\xi_{A}^{*}\left(\underline{r}^{\prime \prime}, \omega ; \underline{r}^{\prime}, t\right) \xi_{B}\left(\underline{r}^{\prime \prime}, \omega ; \underline{r}^{\prime}, t\right)\right] .
\end{gathered}
$$

As in the simple two-beam system, the output gives a measure of the correlation between the $A$ and $B$ components of the light. Using Eqs. (II.23) to express the result in terms of the source distribution gives

$$
\begin{aligned}
& \mathbf{Y}(t)=\frac{2}{\pi} \int \mathrm{d}^{3} \mathbf{r}^{\prime} \int_{0}^{\infty} \mathrm{d \omega}|\boldsymbol{r}(\omega)|^{2} \int_{\substack{\text { (spectrameter } \\
\text { entrance) }}}^{\mathrm{d}^{2} \mathbf{r}^{\prime \prime}} \\
& \text { - } \operatorname{Re}\left[\phi_{A}^{*}\left(\underline{\underline{r}}^{\prime \prime}, \underline{\underline{r}}^{\prime}, \infty\right)_{\theta}{ }^{*}\left(\underline{\underline{r}}^{\prime}, \omega\right) \phi_{B}\left(\underline{\underline{r}}^{\prime \prime}, \underline{\underline{r}}^{\prime}, \omega\right) \theta\left(\underline{\underline{r}}^{\prime}, \omega\right)\right] \\
& =\int z^{3} r^{\prime} \int d \omega|f(\omega)|^{2} \cdot T\left(\underline{r}^{\prime}, \omega\right) d\left(\omega ; \underline{r}^{\prime}, t\right)
\end{aligned}
$$

vhere, as before,

$$
\delta\left(\omega ; \underline{r}^{\prime}, t\right)=g^{*}\left(\underline{x}^{\prime}, \omega ; t\right) B\left(\underline{r}^{\prime}, \omega ; t\right)=\left|s\left(\underline{\underline{x}}^{\prime}, \omega ; t\right)\right|^{2}
$$

16 the spectrum of light emltted at $\underline{r}^{\prime}$. So the output signal

$\mathbf{Y}(t)$ depends upon the quantity

$$
T\left(\underline{r}^{\prime}, \omega\right)=R e \frac{2}{\pi} \int_{\substack{\text { (日pectrowter } \\ \text { ontrance) }}} d^{2} \underline{r}^{\prime \prime} \phi_{A}^{*}\left(\underline{r}^{\prime \prime}, \underline{\underline{r}}^{\prime}, \omega\right) \phi_{B}\left(\underline{\underline{r}}^{\prime \prime}, \underline{\underline{r}}^{\prime}, \omega\right) .
$$


This is the transwission function for the system as a whole. (This term seems approprlate, since the quantity is real, rut it should be noted that this transwission function may be negutive.)

Equation (II.24) is a generalization of Eq. (II.19). Comparing the two results, we see that the simple two-beam system 18 described by

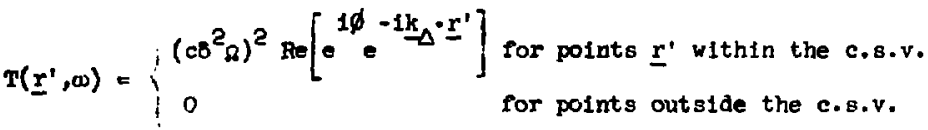

A two-keam spectrameter would olserve the ${ }_{-\Delta}$ component of the distribution of cammon sources. But th1s is Just one spec1si cese of the more general system shown in Fig. II-6. In general, any such arrangement would define some function $T\left(r^{\prime}, \omega\right)$. AccordIng to Eq. (IT.24), the output of the system 18 then given by an integrel over points $\underline{I}^{\prime}$ of the expression $T\left(\underline{r}^{\prime}, \omega\right) A\left(\omega ; \underline{I}^{\prime}, t\right)$. So any system of this type vould select fust one component of the light source distribution: the $T\left(\underline{r}^{\prime}, \omega\right)$ component of $\dot{\alpha}\left(\omega ; \underline{r}^{\prime}, t\right)$. Wany such systews are possible. In Appendix $\mathrm{C}$ of th1s discussion, number of different multiple-beam spectrometers are proposed. In the next section of the present chapter, the mathemet1cal tochnlquos just outlined are applied to several of those same examples.

\section{Three Part 1cular Opt1cel Systems}

In using this description, it is convenlent to first simplify the expressions for the optical path lengths. Treating 
$\underline{x}^{\prime}, x^{(\text {in })}$, and $y^{(i n)}$ as wall quantities, sssuring $z^{(\text {In })}$ nesrly equal to $f_{1}$, and keeping all sall quantities through second oxder glves

$$
\begin{gathered}
\left.\left|\underline{x}^{(1 n)}-\underline{x}^{\prime}\right|+\varphi_{1} \underline{\underline{x}}^{(\text {in })}\right) \simeq \phi_{10}+z^{(\text {In })}-z^{\prime}+\frac{x^{\prime 2}+y^{\prime^{2}}}{2 r_{1}} \\
-\frac{x^{(\ln ) x^{\prime}+y^{(\ln )} y^{\prime}}}{r_{1}} .
\end{gathered}
$$

Keeping leading terms in the magnitude, and corrections in the phace through eecond order, Eq. (II.2O) becomes

$$
t_{0}\left(\underline{x}^{(\mathrm{fn})}, t\right)=\left.\frac{1}{I_{1}} \int d^{3} r^{\prime} s\left(\underline{x}^{\prime}, t^{\prime}\right)\right|_{R e t .}
$$

Bet.: $t^{\prime}=t-\frac{1}{c}\left(\phi_{10}+z^{(\ln )}-z^{\prime}+\frac{x^{\prime 2}+y^{\prime 2}}{2 f_{1}}\right.$

$$
-\frac{x^{(1 n)} x^{\prime}+y^{(1 n)} y^{\prime}}{f_{1}}
$$

In all of the systoms which we shall consider, the first lens 18 followed by a calcite rhoml. Th1s element displeces one polarizetion component by distance d, while leaving the other unaffected. (Beo Appendix C.) Thus, after the rhomb (at points $\tilde{\underline{Y}}), t_{0} 18$ spilt into two waves.

$$
\begin{aligned}
s_{A}(\tilde{\underline{x}}, t) & =\left.s_{0}\left(\underline{r}^{(1 n)}, t^{(1 n)}\right)\right|_{t} \underline{t}^{(1 n)}=\underline{\tilde{r}}-\hat{e}_{z} D \\
& =\frac{1}{F_{1}} \int d^{3} r^{\prime} s\left(\underline{r}^{\prime}, t-\tau_{A}\right)
\end{aligned}
$$




$$
\begin{aligned}
& c_{A}=\tau_{A}+\frac{1}{c}\left|\phi_{10}+\tilde{z}-D \cdot z^{\prime}+\frac{x^{2}+y^{\prime 2}}{2 f_{1}}-\frac{\tilde{x} x^{\prime}+\tilde{y} y^{\prime}}{f_{1}}\right| \\
& \left.\xi_{B}(\underline{\underline{r}}, t)=\xi_{0} \underline{\underline{r}}^{(\ln )}, t^{(\ln )}\right) \mid \begin{array}{l}
\underline{\underline{r}}^{(\ln )}=\tilde{\underline{r}}-\hat{e}_{y} d-e_{z} D \\
t^{(\ln )}=t-\tau_{B}
\end{array} \\
& =\frac{1}{\mathbf{f}_{1}} \int \mathrm{d}^{3} \boldsymbol{r}^{\prime} \mathrm{s}\left(\underline{\mathbf{r}}^{\prime}, t-c_{B}\right)
\end{aligned}
$$

$c_{B}=\tau_{B}+\frac{1}{c}\left(\phi_{10}+\tilde{z}-D-z^{\prime}+\frac{x^{\prime^{2}}+y^{\prime 2}}{2 f_{1}}-\frac{\tilde{x} x^{\prime}+(\tilde{y}-d) y^{\prime}}{f_{1}}\right)$.

Here $\tau_{A}$ and $\tau_{B}$ are delays due to the rhomb, and $D$ is the length of the rhomb.

We shall. aisc need the derlvat Ives

$$
\begin{aligned}
& \nabla \xi_{A, B}(\tilde{\underline{r}}, t)=\frac{1}{\vec{F}_{1}} \int d^{3} r^{\prime} \frac{1}{c} \mid \hat{e}_{x} \frac{x^{\prime}}{I_{1}}+\hat{e}_{y} \frac{y^{\prime}}{I_{1}}-\hat{e}_{z} \\
& \text { - }\left.\frac{\partial}{\partial t^{\prime}} s\left(\underline{r}^{\prime}, t^{\prime}\right)\right|_{t^{\prime}}=t-c_{A, B}
\end{aligned}
$$

and

$\frac{\partial}{\partial t} \xi_{A, B}(\underline{\underline{r}}, t)=\left.\frac{1}{\underline{r}_{1}} d^{3} \mathbf{r}^{\prime} \frac{\partial}{\partial t^{\top}} s\left(\underline{r}^{\prime}, t^{\prime}\right)\right|_{t^{\prime}=t-c_{A, B}}$

Tho simplest system vich we shall consider is one in which the rhomb is followed by a mak with a single slit, as shown in Fig. C-4 in Appendix C. In this case, 


$$
\left.\xi_{A, B} \underline{r}^{\text {(out) }}, t\right)\left\{\begin{aligned}
= & \left.\xi_{A, B}(\underline{\underline{r}, t})\right|_{\tilde{\underline{r}}}=\underline{r}^{\text {(out) }} \\
& \text { for } \tilde{x}_{1}<x^{\text {(out) }}<\tilde{x}_{2} \\
& \tilde{y}_{1}<y^{\text {(out) }}<\tilde{y}_{2} \\
=0 & \text { otherwise. }
\end{aligned}\right.
$$

From the decussion in Appendix C, it is expected that the effect of this arrangement should resemble that. of a simple two-beam Bystem.

Equations (II.22) can also be simplified. Assuming that $x^{\text {(out) }}, y^{\text {(out) }}, x^{\prime \prime}, y^{\prime \prime}$, and $\left(z^{\prime \prime}-z^{\text {(out) }}-f_{2}\right.$ ) are all small quantities, one can approximate the last segment of the path,

$$
\begin{aligned}
& \left.\left|\underline{r}^{\prime \prime}-\underline{r}^{\text {(out })}\right|+\phi_{2} \underline{r}^{\text {(out) })}\right) \approx \phi_{20}+z^{\prime \prime}-z^{\text {(out) })}+\frac{x^{\prime \prime}+y^{\prime \prime}}{2 f_{2}} \\
& +\frac{x^{(\text {out })} x^{\prime \prime}+y^{\text {(out })} y^{\prime \prime}}{t_{2}} .
\end{aligned}
$$

Using this approximation, and the preceding expressions for $\nabla_{\xi}$ and $\partial \xi / \partial t$, the two field amplitudes, $\xi_{A, B}\left(\underline{r}^{\prime \prime}, t\right)$ as the spectrometer entrence slit [given by Eq. (II.22)] may be written,

$$
\begin{aligned}
& s_{A, B}\left(\underline{\underline{r}}^{\prime \prime}, t\right) \tilde{=}-\frac{1}{2 \pi c} \frac{1}{\mathrm{I}_{1}} \frac{1}{\mathrm{f}_{2}} \int_{\tilde{x}_{1}}^{\tilde{x}_{2}} \mathrm{~d} \tilde{x} \int_{\tilde{y}_{1}}^{\tilde{y}_{2}} \mathrm{~d} \tilde{y} \int \mathrm{d}^{3} r^{\prime} \\
& \text { - }\left.\frac{\partial}{\partial t^{\prime}} s\left({\underline{I^{\prime}}}^{\prime}, t^{\prime}\right)\right|_{t^{\prime}}=\dot{\tau}-c_{A, B}-c_{0}
\end{aligned}
$$




$$
c_{0}=\frac{1}{c} i_{20}+\phi^{\prime \prime}-\tilde{z}+\frac{x^{\prime \prime 2}+y^{\prime \prime 2}}{2 f_{2}}-\frac{\tilde{x} x^{\prime \prime}+\tilde{y} y^{\prime \prime}}{f_{2}}
$$

To obtain the transfer functions $\oint_{A, B}$, one must then Fourier transform this expression

$$
\begin{aligned}
& \xi_{A, B}\left(\underline{r}^{n}, \omega\right)=\frac{-1}{2 \pi c} \frac{1}{f_{1}} \frac{1}{f_{2}} \int_{\tilde{x}_{1}}^{\tilde{x}_{2}} d \tilde{x} \int_{\tilde{y}_{1}}^{\tilde{y}_{2}} d \tilde{y} \int a^{3} r^{\prime} j d t e^{i j t} \\
& \text { - }\left.\frac{\partial}{\partial t^{\prime}} s\left(\underline{r}^{\prime}, t^{\prime}\right)\right|_{t^{\prime}=t-c_{A, B}-c_{O}} \\
& =\frac{-1}{2 \pi c} \frac{1}{r_{1}} \frac{1}{f_{2}} \int_{\tilde{x}_{1}}^{\tilde{x}_{2}} d \tilde{x} \int_{\tilde{y}_{1}}^{\tilde{y}_{2}} d \tilde{y}, d^{3} r^{\prime} \text { ieve }+\operatorname{i\omega s}\left(c_{A, B}+c_{0}\right) s\left(\underline{r}^{\prime}, \omega\right) .
\end{aligned}
$$

Th1s has the needed form Eq. (II.?3) :

$$
\xi_{A, E}\left(\underline{r}^{\prime \prime}, \omega\right)=\int d^{3} \underline{r}^{\prime} \phi_{A, B}\left(\underline{r}^{\prime \prime}, \underline{r}^{\prime}, \omega\right) B\left(\underline{r}^{\prime}, \omega\right) \text {. }
$$

Evaluating the $\tilde{x}$ and $\tilde{y}$ integrations gives,

$$
\begin{aligned}
& \left.\phi_{A, B}\left(\underline{r}^{\prime \prime}, \underline{\underline{x}}^{\prime}, \omega\right)=\frac{+1}{2 \pi}\left(\frac{c}{\omega}\right) \frac{1}{f_{1}} \frac{1}{f_{2}} \frac{1}{\left(y^{\prime} / f_{1}+y^{\prime \prime} / f_{2}\right)} \frac{1}{\left(x^{\prime} / \underline{x}_{1}+x^{\prime \prime} / f_{2}\right.}\right) \\
& \left\{e^{+i \omega\left[c_{A, B}\left(\tilde{x}_{2}, \tilde{y}_{2}\right)+c_{0}\left(\tilde{x}_{2}, \tilde{y}_{2}\right)_{1}\right.}\right. \\
& -e^{+10\left[c_{\Lambda,},\left\{\tilde{x}_{1}, \tilde{y}_{2}\right)+c_{0}\left(\tilde{x}_{1}, \tilde{y}_{2}\right)\right]} \\
& -e^{\left.+1 \omega_{[A, B}^{[} c_{A}\left(\tilde{x}_{2}, \tilde{y}_{1}\right)+c_{0}\left(\tilde{x}_{2}, \tilde{y}_{1}\right)\right]} \\
& +e^{\left.+10\left[c_{A, B}\left(\tilde{x}_{1}, \tilde{y}_{1}\right)+c_{0}\left(\tilde{x}_{1}, \tilde{y}_{1}\right)\right]\right\} \text {. }}
\end{aligned}
$$




$$
\begin{aligned}
& T\left(\underline{r}^{\prime}, \omega\right)=\operatorname{Re} \frac{2}{\pi} \int c^{2} r^{\prime \prime} \phi_{A}^{*} \phi_{B} \\
& =\operatorname{Re}\left(\frac{2}{\pi}\left(\frac{\omega}{c}\right)^{2} \frac{1}{\left(f_{1} f_{2}\right)^{2}} e^{+1 \omega\left(\tau_{B}-\tau_{A}\right)} e^{+1\left[(\omega / c)\left(d y^{\prime} / f_{1}\right) !\right.}\right. \\
& \cdot \int_{x_{1}^{\prime \prime}}^{x_{2}^{\prime \prime}} d x^{\prime \prime} \int_{y_{1}^{\prime \prime}}^{y^{\prime \prime}} d y^{\prime \prime} \frac{1}{x^{2}} \frac{1}{y^{2}} \\
& \sin ^{2}\left(\frac{x \tilde{x}_{2}-x \tilde{x}_{1}}{2}\right) \sin ^{2}\left(\frac{x \tilde{y}_{2}-\tilde{y}_{1}}{2}\right)
\end{aligned}
$$

where

$$
\begin{aligned}
& X=\frac{\omega}{c}\left(\frac{x^{\prime}}{f_{1}}+\frac{x^{\prime \prime}}{f_{2}}\right) \\
& Y=\frac{\omega}{c}\left(\frac{y^{\prime}}{f_{1}}+\frac{y^{\prime \prime}}{f_{2}}\right)
\end{aligned}
$$

and $\left(x_{2}^{\prime \prime}-x_{1}^{\prime \prime}\right)$ and $\left(y_{2}^{\prime \prime}-y_{1}^{\prime \prime}\right)$ are the dimensions of the spectrometer entrance alit. The width $\left(y_{2}^{\prime \prime}-y_{1}^{\prime \prime}\right)$ is assumed to be less than the width of a max.mun of a diffraction pattern due to the flrst slit. (See Appendix C.) Thus $Y$ may be considered constent in the integration over $y^{\prime \prime}$. However, $\left(x_{2}^{\prime \prime}-x_{1}^{\prime \prime}\right)$, the length of the entrance sl1t, 18 much larger than the width of $1 / x^{2}$, so the $x "$ Integration may as well be taken over the whole line. Making these replacementa gives,

$$
\begin{gathered}
T\left(\underline{r}^{\prime}, \omega\right)=\operatorname{Re}\left(\frac{2}{\pi}\right)^{2} \frac{\omega}{c} \frac{1}{\left(f_{1} f_{2}\right)^{2}} e^{+1 \omega\left(\tau_{B^{-}} \tau_{A}\right)} f_{2}\left(y_{2}^{\prime \prime}-y_{1}^{\prime \prime}\right)\left(\tilde{x}_{2}-\tilde{x}_{1}\right) \\
e^{+1\left[(\omega / c)\left(\partial y^{\prime} / f_{1}\right)\right]_{F\left(y^{\prime}\right)}}
\end{gathered}
$$

where 


$$
F\left(y^{i}\right)=\frac{\sin ^{2}\left|y \frac{\left(\tilde{y}_{2}-\tilde{y}_{1}\right)}{2}\right|}{y^{2}}
$$

defines the source region observed. Assuming that the source is incoherent, one can used Eq. (II.24) to write the output of the system,

$$
\begin{aligned}
& \mathbf{r}(t)=\int d^{2} r^{\prime} \int d \omega|f(\omega)|^{2} T\left(\underline{r}^{\prime}, \omega\right) \&\left(\omega ; \underline{r}^{\prime}, t\right) \\
& =\left.c \int_{\alpha \omega}\left|\frac{\omega}{c}\right| f(\omega)\right|^{2} \operatorname{Re} e^{+\operatorname{t\omega }\left(\tau_{\left.B^{-\tau_{A}}\right) !} a^{3} r^{\prime}\right.} F\left(y^{\prime}\right) \\
& e^{+1\left[(\omega / c)\left(d y^{\prime} / f_{1}\right)\right]} ;\left(\omega ; \underline{s}^{\prime}, t\right) \\
& =\left.C \int d \omega\left|\frac{\omega}{c}\right| f(\omega)\right|^{2} \text { Re } e^{+\operatorname{lor}\left(\tau_{B}-\tau_{A}\right)} \sim_{\text {observed }}^{f}\left(\omega ; \underline{k}_{\Delta}, t\right)
\end{aligned}
$$

where

$$
\begin{aligned}
& c=\left|\frac{2}{\pi}\right|^{2} \frac{1}{\left(f_{1} f_{2}\right)^{2}}\left(\tilde{x}_{2}-\tilde{x}_{1}\right) f_{2}\left(y_{2}^{\prime \prime}-y_{1}^{\prime \prime}\right) \\
& \mathcal{L}_{\text {obuerved }}\left(\omega ; \underline{r}^{\prime}, t\right)=A\left(\omega ; \underline{r}^{\prime}, t\right) F\left(y^{\prime}\right)
\end{aligned}
$$

and

$$
\underline{k}_{\Delta}=-e_{y} \frac{\omega}{c} \frac{d}{f_{1}} .
$$

So, assuming that $e^{+1 \omega\left(\tau_{B}-\tau_{A}\right)}$ is constant over the range of frequencies considered (which is equivalent to saying that the path difference is less than the coherence length) this system observes the $k_{\Delta}$ spatial Fourler component of the distribution of light sources within the observetion region. Thus, at least near 
the focal plane of the first lens, this setup is equivelent to the simple two-beam gystem which was discussed in section II.A. Moreover, the result is seen to depend upon the width, $\left(\tilde{y}_{2}-\tilde{y}_{1}\right)$ of the aperture in the mask behind the rhomb, but not upon its location, since $\tilde{y}_{1}$ and $\tilde{y}_{2}$ could both be changed by a conmon amount and not affect the answer. This suggests using a mask with many slits, as shown in Fig. C-6a in Appendix C. This second system would give two transfer functions, [for n slits, separated by a distance $\left.\Delta<\left(\tilde{y}_{2}-\dot{y}_{1}\right)\right]$

$$
\begin{aligned}
& \phi_{A, B}\left(\underline{\underline{x}}^{\prime \prime}, \underline{\underline{r}}^{\prime}, \omega\right)=\frac{-1 \omega}{2 \pi c} \frac{1}{\mathrm{f}_{1}} \frac{1}{\underline{f}_{2}} \int_{\tilde{x}_{1}}^{\tilde{x}_{2}} d \tilde{x} \sum_{j=0}^{n} \int_{\tilde{y}_{1}+j \Delta}^{\tilde{y}_{2}+j \Delta} d \tilde{y} e^{+1 \omega\left(c_{A, B}+c_{0}\right)} \\
& =\frac{-1 \omega}{2 \pi c} \frac{1}{\mathrm{f}_{1}} \frac{1}{\mathrm{f}_{2}} \mathrm{e}^{+i \omega, \mathrm{A}, \mathrm{B}} \mathrm{e}^{+1(\omega / c) \otimes} \int_{\tilde{x}_{1}}^{\tilde{x}_{2}} \mathrm{~d} \tilde{\mathrm{x}} \mathrm{e}^{-1 \mathrm{x} \tilde{\mathrm{x}}} \\
& \cdot \sum_{j=0}^{n} \int_{\tilde{y}_{1}+j \Delta}^{\tilde{y}_{2}+j \Delta} d \tilde{y} e^{-1 \tilde{y}}\left\{\begin{array}{l}
1 \text { for } A \\
e^{+1(\omega / c)\left(d y / / f_{1}\right)} \text { for } B
\end{array}\right. \\
& =\frac{-1 \omega}{2 \pi c} \frac{1}{I_{1}} \frac{1}{f_{2}} \frac{1}{\bar{x}} \frac{1}{\bar{Y}} e^{+1 \omega \tau} A, B e^{+i(\omega / c) \Phi}\left(e^{-1 x \tilde{x}_{2}}-e^{-1 x \tilde{x}_{1}}\right) \\
& \sum_{j=1}^{n} e^{-1 j \Delta y}\left(e^{-1 Y \tilde{y}_{2}}-e^{-1 Y \tilde{y}_{1}}\right)\left\{\begin{array}{l}
1 \text { for } A \\
\cdot e^{+1(\omega / c)\left(d y / / f_{1}\right) \text { for } B}
\end{array}\right.
\end{aligned}
$$

where

$$
\nabla=\phi_{10}+\phi_{20}+z^{\prime \prime}-z^{\prime}-D+\frac{x^{\prime 2}+y^{\prime 2}}{2 f_{1}}+\frac{x^{\prime \prime 2}+y^{\prime \prime 2}}{2 f_{2}} .
$$


Uaing these to calculate the tranamisaion fuction for th1s gyatem gives,

$$
\begin{aligned}
& T\left(\underline{I}^{\prime} \omega\right)=\left.\operatorname{Re} i \frac{2}{\pi}\right|^{3}\left|\frac{\omega}{c}\right|^{2} \frac{1}{\left(\rho_{1} f_{2}\right)^{2}} e^{+1 \omega\left(\tau_{B}-\tau_{A}\right)} e^{+1\left[(\omega / c)\left(d y^{1} / f_{1}\right)\right]} \\
& j_{x_{1}^{\prime \prime}}^{x_{2}^{\prime \prime}} d x^{\prime \prime} \frac{1}{x^{2}} \sin ^{2} \frac{x \tilde{x}_{2}-x \tilde{x}_{1}}{2} j_{y_{1}^{\prime \prime}}^{y_{2}^{\prime \prime}} d y^{\prime \prime} \frac{1}{y^{2}} \\
& \frac{\sin ^{2}\left(\frac{\tilde{Y y}_{2}-\tilde{y}_{1}}{2}\left|\sin ^{2}\right| \frac{n \Delta y}{2}\right)}{\sin ^{2}\left(\frac{\Delta y}{2}\right)}
\end{aligned}
$$

Assuming aga1n that $\left(x_{2}^{\prime \prime}-x_{1}^{\prime \prime}\right) 15$ large, while $\left(y_{2}^{\prime \prime}-y_{1}^{\prime \prime}\right)$ is small, one hes,

$$
\begin{aligned}
T\left(I^{\prime}, \infty\right)= & \operatorname{Re} C \frac{\omega}{c} e^{+\operatorname{L\omega }\left(\tau_{\left.B^{-}-\tau_{A}\right)}\right.} e^{+1\left[(a / c)\left(d y^{\prime} / s_{1}\right)\right]} \\
& \cdot F\left(y^{\prime}\right)\left[\frac{\sin ^{2}\left(\frac{\Delta Y \Delta}{2}\right)}{\left.\sin ^{2} \mid \frac{Y \Delta}{2}\right)}\right]
\end{aligned}
$$

wsth $Y$ evelusted at $y_{1}^{\prime \prime}$.

This result 18 scwowhat similer to that found for the oimple two-boem oystom, but there 18 an important oxtre factor

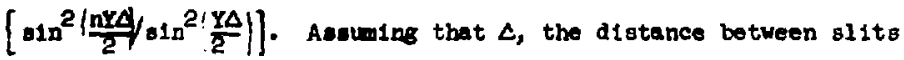
Ia comparbiblo to $d$, the diaplecowent due to the rhomb, and that $y=\omega / c\left[\left(y^{\prime} / t_{1}\right)+\left(y_{1}^{\prime \prime} / f_{2}\right)\right]$ is of the same order as $(\omega / c)\left(y^{\prime} / t_{1}\right)$, the edditional rector $\sin ^{2}(n Y \Delta / 2)$ will vary much mare rapldily with $y^{\prime}$ then will the exponentiel, $+\left\{\left[(a / c)\left(d y^{\prime} / f_{1}\right)\right.\right.$. Thus the 
effer: of such an apparatus would be very different from that of a simple two-beam system. The multiple slit arrangement would observe exclusively those regions where the extra factor is large. As is explained in Appendix C, this difference in effect is cussed by interference between light observed through different Blits in the first maks. Because of this additional interference, the result is not just a sum of two-beam ouservations. To make a more efficient version of the two-beam Eystem, one must avold thle Interference between separate pairs of beams. As is suggeated in Appendix C, one can do tilis by using a colliwator to select the Iight from each patr of beams inde!endently, before the polnt where the separate pairs of beams are all combined. Such an apparatus 18 shown in Fig. C-7 in Appendix C.

To describe this third arrangement, we denote points at the output of the collimator $\underline{\underline{r}}=(\vec{x}, \tilde{y}, \tilde{z})$. The light there con* B1ste of outputs frow the different collimator slits,

$$
s_{A, B}(\underline{\underline{y}}, t)=\left\{\begin{array}{lc}
t_{A, B}^{(g)}(\underline{x}, t) & \text { for } \tilde{x}_{1}<\tilde{x}<\tilde{x}_{2} \\
0 & \tilde{y}_{1}+j \Delta<\tilde{y}^{2}<\tilde{y}_{2}+j \Delta \\
\text { otherwise. }
\end{array}\right.
$$

The output of each slit is Given by a K1rchoff Integral across the entrance to the section of the collimator

$$
\begin{aligned}
& s_{h, B}^{(j)}(\underline{x}, t)=\frac{1}{4 \pi} \int_{\tilde{x}_{1}}^{\tilde{x}_{2}} d \tilde{x} \int_{\tilde{y}_{2}+j \Delta}^{\tilde{y}_{2}+j \Delta} d \tilde{y} \frac{1}{|\underline{\underline{r}} \cdot \underline{\underline{r}}|} \\
& \text { - }\left[\left.\nabla_{\xi_{A, B}}(\tilde{\tilde{r}}, \tilde{t})\right|_{z}-\frac{1}{c} \frac{\partial}{\partial \tilde{t}} s_{A, B}(\underline{\tilde{r}}, \tilde{t})\right]_{\tilde{t}=t}-\frac{|\underline{\tilde{r}}-\underline{\tilde{r}}|}{c}
\end{aligned}
$$




$$
\begin{aligned}
& =\frac{1}{2 \pi} \int_{\tilde{x}_{1}}^{\tilde{x}_{2}} d \tilde{x} \int_{\tilde{y}_{1}+j \Delta}^{\tilde{y}_{2}+j \Delta} d \tilde{y} \frac{1}{L} \frac{-1}{c} \frac{1}{r_{1}} d^{3} r^{\prime} \\
& \cdot \frac{\partial}{\partial t},\left.s\left(\underline{r}^{\prime} t\right)\right|_{t^{\prime}=t-c_{A, \nu}} \tilde{c}
\end{aligned}
$$

where

$$
\tilde{c}=\frac{1}{c}\left\{\tilde{z}-\tilde{z}+\frac{I}{2 I}\left[(\tilde{x}-\tilde{x})^{2}+(\tilde{y}-\tilde{y})^{2}\right]\right\}
$$

and I $1 \mathrm{~s}$ the length of the collimator. Here Eqs. (II.26́a,b) have been used to express $t$ e field $\xi(\underline{\tilde{\tilde{F}}}, t)$ in terms of the source $\theta\left(\underline{I}^{\prime}, t^{\prime}\right)$ and the distances $(\tilde{x}-\tilde{x}),(\tilde{y}-\tilde{y})$, and $\left.\tilde{z}-\tilde{z}-L\right)$ have been treated as emall quantities in an expansion of the path Iength.

The fields at the sper ieter entrance slit are a sum of contributions frow the different collimator slit-, contributions given by a Kirchoff integral across the sollimator exit:

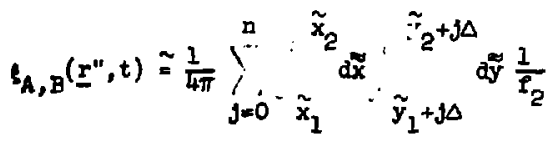

$$
\begin{aligned}
& \cdot\left[\left.\nabla_{\xi}^{(j), B}(\tilde{I}, \tilde{t})\right|_{z}-\frac{1}{c} \frac{\partial}{\partial \tilde{t}} \xi^{(j)}(\tilde{\tilde{r}}, \tilde{t})\right]_{\tilde{t}=t}-c_{0}^{\prime}
\end{aligned}
$$

where

$$
c_{0}^{\prime}=\frac{I}{c} \phi_{20}+z^{\prime \prime}-z+\frac{x^{\prime \prime}+y^{\prime 2}}{2 x_{2}} \cdot \frac{\not y x^{\prime \prime}+y_{y^{\prime \prime}}}{I_{2}} ! \cdot
$$


Using the ebove expressions for $\xi^{(j)}(\underline{\underline{F}}, t)$, and Fourter transforming the result gives for this system the two transfer functions,

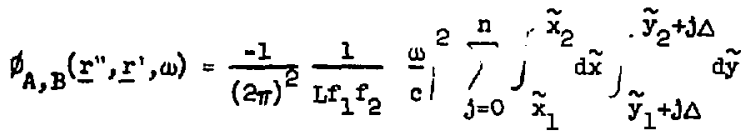

$$
\begin{aligned}
& \cdot \int_{\tilde{x}_{1}}^{\tilde{x}_{2}} d \tilde{x} \int_{\tilde{y}_{1}+j \Delta}^{\tilde{y}_{2}+j \Delta} \tilde{a \tilde{y}} e^{\operatorname{tar}\left(c_{A, B}+\tilde{c}+c_{j}^{\prime}\right)} \\
& =\frac{1}{(2 \pi)^{2}}(1+1) \cdot \sqrt{\frac{\pi L c}{\omega}} \frac{1}{L y^{\prime} y^{\prime \prime}} e^{1 \omega \tau A, B} e^{1(\omega / c) \phi} \\
& \cdot e^{-1(\omega / c)(L / 2)\left(x^{\prime} / f_{1}^{2}\right)} \frac{1}{x} j^{-1 x \tilde{x}_{2}}-e^{-1 x \tilde{x}_{1}} \\
& \cdot \sum_{j=0}^{n} e^{-1\left[(\omega / c)\left(y^{\prime} / f_{1}\right) j \Delta\right]_{e}-1 \mid(\omega / c)\left(y^{\prime \prime} / f_{2}\right) j \Delta !} \\
& \text {. } e^{-1(a / c)\left(y+\tilde{y}_{2} / f_{1}\right)}-e^{-1(\omega / c)\left(y^{\prime} \tilde{y}_{1} / f_{1}\right)} \\
& \text { - }\left\{\begin{array}{l}
e^{-1(\omega / c)\left(y " \tilde{y}_{2} / f_{2}\right)}-e^{-1(\omega / c)\left(y^{\prime \prime} \tilde{y}_{1} / f_{2}\right)} \\
e^{1\left(d y / f_{1}\right)(\omega / c)} \text { for } A
\end{array}\right.
\end{aligned}
$$

uhare in the $x$ and $y$ integrations it bae been assumed that $\left(x_{2}-x_{1}\right.$ ) 1. large, while $\left(y_{2}-y_{1}\right)$ is small in comparison with the widths of the ropective integrand $\mathrm{B}$.

Combl. s these tun transfer functions elvas the transwienton runction for this oy atem: 


$$
\begin{aligned}
& T\left(\underline{r}^{\prime}, \omega\right)=\operatorname{Re} \frac{2}{\pi} \int d^{2} r^{\prime \prime} \phi_{A}^{*} \phi_{B} \\
& =\operatorname{Re}\left(\frac{2}{\pi}\right)^{4} \frac{1}{\mathrm{~L}} \frac{c}{\omega} e^{\operatorname{t\omega }\left(\tau_{B}-\tau_{A}\right)} e^{1\left(d y^{\prime} / f_{I}\right)(\omega / c)} j d^{2} r^{\prime \prime} \\
& \cdot \frac{1}{x^{2}} \sin ^{2}\left(\frac{x \tilde{x}_{2}-x \tilde{x}_{1}}{2}\right) \frac{1}{\left(y^{\prime} y^{\prime \prime}\right)^{2}} \\
& \text { - } \sin ^{2}\left[\frac{\omega}{c} \frac{y^{\prime}}{f_{1}} \frac{\left(\tilde{y}_{2}-\bar{y}_{1}\right)}{2}\right] \sin ^{2}\left[\frac{\omega y^{\prime \prime}}{c} \frac{\left(\tilde{y}_{2}-\tilde{y}_{1}\right)}{2}\right] \\
& \cdot \sum_{j=0}^{n} \sum_{j=0}^{n} e^{-1 Y\left(j-j^{\prime}\right) \Delta}
\end{aligned}
$$

The reason for using a collimator is to avold any ef. ict of multiple-beam interference between light accepted through differeat slits in the collimator. For this reason, it is essential that no more 11ght should be rejected after all the beams bave been combined. Thus the spectroneter entrance olit should be made larger than the width of the lllumination pattern there, and in the above exprossion the $\underline{r}^{\prime \prime}$ integration should be taken over the shole plane. Doing this one has,

$$
\begin{gathered}
T\left(\underline{r}^{\prime} \omega\right)=R \theta\left(\frac{2}{\pi}\right)^{2} \frac{1}{L} \frac{c}{\omega} \Delta\left(\tilde{x}_{2}-\tilde{x}_{1}\right)\left(\tilde{y}_{2}-\tilde{y}_{1}\right) e^{\operatorname{l\omega }\left(\tau_{B}-\tau_{A}\right)} \\
\cdot e^{1\left(d y \cdot / f_{1}\right)(\omega / c)} \frac{1}{y^{\prime}} \sin ^{2}\left[\frac{\omega y^{\prime}}{c} \frac{\left(\tilde{y}_{2}-\tilde{y}_{1}\right)}{2}\right] .
\end{gathered}
$$

Here the doublo sum has vaniobod. Thlo occurs in the $y "$ integret10R, where owe has expressions of the forw, 


$$
\int_{-\infty}^{\infty} d y^{\prime \prime} \frac{1}{y^{\prime \prime 2}} \sin ^{2}\left[\frac{\omega\left(\tilde{y}_{2}-\tilde{y}_{1}\right)}{2} y^{\prime \prime}\right] \cos \left[\frac{\omega \Delta}{c} \frac{\Delta}{c}\left(j-j_{2}\right) y^{\prime \prime}\right] \cdot
$$

This vanishes ${ }^{4 l}$ for $J \neq J^{\prime}$, provided that $\Delta>\left(y_{2}-y_{1}\right)$, 1.e., provided that the slit separation exceeds the ollt width, which 1t obviously does. This leaves a single sum of identical tems, which gives the factor $n$.

Thus the effect of interference vanishes, as was expected, and the contributions fram the different slits are seen to be 1deatiral, because, as noted ear:fer, each result is independent of the slit position. So the conclusion or Appendix $\mathrm{C}$ is veriIled: A spectrometer with many independently collimated pairs of beams provides a more efflcieat version of the simple twobeam aystem wh1ch was f1rst cons1dered. It should be remembered, bowever, that this is true only of reglons near the focus of the Bystom.

\section{Eigher Order Correlaticns}

the elementary two-beam ipectrometer discussed in section II.A would give an output, $Y(t)$, proportional to the mutual coberence $\Gamma_{B A}(0 ;|\underline{k}|, t)$ between light of vavelength $2 \pi|\underline{k}|^{-1}$ emitted In directions $\hat{k}_{A}$ and $\hat{k}_{B}$ trom the same region. In the snalysis In section II .3, this quantity use shoun to be proportional to on pourior componant, $\mathbb{d}\left(|\underline{k}| c_{j} k_{\Delta}, t\right)$, of tho alstribution of obsorved light sources. According to the Wiener-Khintehino theorew, ${ }^{21}$ meavurument of $A\left(|\underline{\underline{k}}| c ; \underline{k}_{\Delta}, t\right)$ is equivalont to measurement of the two-point correlation tunction or the light source distribution. 
Hence a two-beam spectroscopic measurement of correlations in the Ifght emitted by a plasma would give information about twopoint correlations in the disi.fbution of the source. So stated, this result suggests that there may be a much more general correspondence between correlation functions of a light fleld, $\xi(\underline{r}, t)$, and correlation functions of the density of sources, $A\left(|\underline{x}| c ; \underline{r}^{\prime}, t\right)$.

In discussions of optical theory, higher order correlations, 11ke the two-beam mutul coherence, are custamarily defined in terms of an astociated analytic signal. Wol $f^{42}$ has used this technique to define a capplete set of complex correlations,

$$
r^{(m, n)} \equiv 2^{(m+n)} \xi_{1}^{(+) * \xi_{2}^{(+) *} \cdots \xi_{m}^{(+) *} \xi_{m+1}^{(+)} \cdots \xi_{m+n}^{(+)}} \cdot \quad(I I .34)
$$

(the use of this form here involves one change, bowever, since the definition was mede to campare light at one point at different tiwes, whlle the otservations discused bere vould ccmpare light at one point in different beams with no delays. ${ }^{43}$ ) similar deflnition. have been used by other authors. (see, for example, Rer. 10.)

We aball not conelder here the waye in which the se bigher order corraletions might bo woncured. It is possible, at lesst It priaciple, to obcerve tbop (for example, by bosuring hlgher onder corralations of intensity), but that problow is beyand the - cope of this lavestigation. However, the theory in section II.A.3 is madily oxtended to some hlgbor orvor quant1tios. B1000 th1s should be of Interest by 1tealf, such an extenetion of the thoory 10 outlined in the next fou paess. 
It is shown in Appendix E. 1 that light of wavelength $2 \pi|\underline{k}|^{-1}$ enftted in direction $\hat{k}$ may be described by a fleld amplitude,

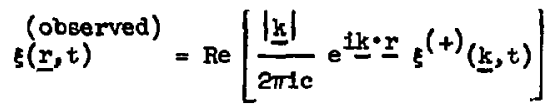

where $g^{(+)}(\underline{k}, t)$ is the positive frequency, or analytic signal associated with the spatial Fourier transform of the fleld. (In this scaler model, the normalization is arbitrary, since the physlcal nature of $\xi$ has not been specified.) The analytic signal associated with this field is

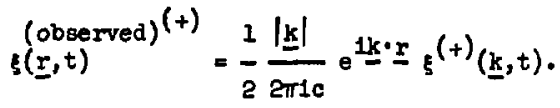

This follows frow the result shown in Appendix E.2. Th1s form Is now to be used in the expressions for the correlation funct1ons.

To rurther simplify the problem, it is conventent to expl1citly assume that the source, $s\left(\underline{x}^{\prime}, t\right)$, is quasimonochromatic;

$$
s\left(\underline{r}^{\prime}, t\right)=2 a\left(\underline{r}^{\prime}, t\right) \cos \left[\omega_{0} t+\phi\left(\underline{r}^{\prime}, t\right)\right] .
$$

The emplitude, $a\left(\underline{I}^{\prime}, t\right)$, 1e proportionel to the square root or the cource density and the phase, $\phi\left(\underline{I}^{\prime} t\right)$, is rendeo from polnt to polat. Tho time veriation of both a and $\phi 18$ assumed to be whoh olower than the oecillation at the opticel frequency, $\omega_{0}$. Ualise the oroen's runction expression for the flold Eq. II.1)] on tase 


$$
\begin{aligned}
& g^{(t)}(\underline{k}, t)=\int d^{3} r e^{-1 \underline{k} \cdot \underline{r}} \int d^{3} r^{\prime} \frac{1}{\left|\underline{\underline{r}}-\underline{\underline{I}}^{\prime}\right|} \mathrm{a}\left(\underline{\underline{r}}^{\prime}, t-\frac{\left|\underline{\mathbf{r}}-\underline{\underline{r}}^{\prime}\right|}{c}\right) \\
& \cdot e^{-1 \omega_{0}\left[t-\left(\left|\underline{r}-\underline{r}^{\prime}\right|\right) / c\right]} e^{-1 \phi\left[\underline{r}^{\prime}, t-\left(\left|\underline{r}-\underline{r}^{\prime}\right|\right) / c\right]} \\
& =\int_{-\infty}^{t} c d \tau \frac{2 \pi}{1 \mid \underline{k} i}\left[e^{1|\underline{k}| c(t-\tau)}-e^{-1|\underline{k}| c(t-\tau)}\right] e^{-1 \omega_{0} \tau}
\end{aligned}
$$

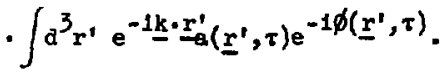

As before, this integration over all preceding times is much 1deallzed. If the finite size of the spectrometer grating is considered, the $\tau$ integration should be taken only over the preceding Inverse optical benduldth. Furthermore, since and $\emptyset$ are slowly varying, they way be taken as constant over such an interval. Frow thls more realistic form one obtaing the result,

$$
\begin{aligned}
s^{(t)}(\underline{k}, t)= & \frac{2 \pi 1 c}{|\underline{k}|} e^{-1|\underline{k}| c t} B\left(\omega_{0}-|\underline{k}| c\right) \int d^{3} r^{\prime} e^{-1 \underline{k} \cdot \underline{r}^{\prime}} a\left(\underline{r}^{\prime}, t\right) \\
& \cdot e^{-1 \phi\left(\underline{r}^{\prime}, t\right) .}
\end{aligned}
$$

Ustug this expression to eveluate the two-beam mutual coberence [1.0., combining Eqs. (II.34) and (II.37)], one bas

$$
\begin{aligned}
& \Gamma^{(1,1)}=4 s_{A}^{(+) * s_{B}^{(+)}} \\
& 0^{-1 k_{A} \cdot \underline{r}_{A}} 0^{1 k_{B} \cdot \underline{r}_{B}} \int d^{3} r^{\prime} \int s_{r^{\prime \prime}} e^{-1 k_{A} \cdot \underline{r}^{\prime}} 0^{1 k_{B} \cdot \underline{r}^{\prime \prime}}
\end{aligned}
$$

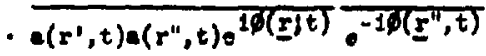




$$
\begin{aligned}
& =e^{-1 \underline{k}_{A} \cdot \underline{r}_{A}} e^{1 \underline{\underline{k}}_{B} \cdot \underline{r}_{B}} \int d^{3} r^{2} e^{-1\left(\underline{k}_{B}-\underline{k}_{A}\right) \cdot \underline{r}^{\prime}} \overline{a^{2}\left(\underline{r}^{\prime}, t\right)} \\
& =e^{-1 \underline{k}_{A} \cdot \underline{r}_{A}} e^{\frac{1 k_{B} \cdot \underline{r}_{B}}{n_{B}\left(\underline{k}_{B}-\underline{k}_{A}, t\right)}}
\end{aligned}
$$

where $n_{g}$ is the (unnormalized) source densits.

Th1s result agrees with that obtained in section II.A.3. But the assumption of Eq. (II.36) has so much sioplifled the derivation that it now can be extended without difficulty to some higher order quantities. For example, one can calculate a four-beam correlation,

$$
\begin{aligned}
& \Gamma^{(2,2)}=16 s_{C}^{(+)^{*} s_{A}^{(+) *} s_{B}^{(+)} s_{D}^{(+)}} \\
& =e^{-1 k_{C} \cdot \underline{r}_{C}} e^{-1 k_{A} \cdot \underline{r}_{A}} e^{1 k_{B} \cdot \underline{r}_{B}} e^{1 k-D \cdot \underline{x}_{D}} \int d^{3} r^{\prime} \int d^{3} r^{\prime \prime}
\end{aligned}
$$

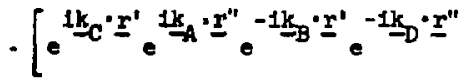

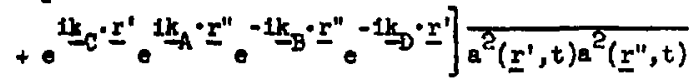

$$
\begin{aligned}
& =e^{-1 k_{C} \cdot \underline{x}_{C}} e^{-1 \underline{k}_{A} \cdot \underline{x}_{A}} e^{1 \underline{k}_{B} \cdot \underline{r}_{B}} e^{1 \underline{k}_{D} \cdot \underline{r}_{D}} \\
& \text { - }\left[\overline{n_{B}\left(k_{B}-k_{C}{ }^{\prime}\right) n_{B}\left(g_{D}-k_{A}, t\right)}+\overline{n_{B}\left(k_{D}-k_{C}, i\right) n_{B}\left(k_{B}-k_{A}, t\right)}\right] .
\end{aligned}
$$

So th1s four-base optical corralntion 1 s seen to be proportiocel to a sum of man products of source density fluetuation compononto. Bore the renalning exponentiel tactors are just conotante. The vource denoity ocoponent s, $n_{g}(k, t)$, are complex relued apetial Fourler trunsforms. Hence the Indicated average producte depond upon the phases of the rectors. If there vere a correletion be ween two such quentities dus, for oxample, to an 
Interaction between plasma waves with wave vectors $\left(\underline{k}_{B}-\underline{k}_{C}\right)$ and $\left(k_{-1}-k_{A}\right)$, the measured averages would $A: P$ fer from a product of the averages of the two factors. Such a correlation corresponds to higher orde:" correlations in the light source distribution. Again, this all assumes an fncoherent source. There is no correlation between the phases of the light from different points. But there can be correlations between the light, ierved through different beams, because the same sources contribute to them all. Th1s was shown to be the case for the two-beam coherence, and the same thing is now seen to be true also of some hitgher order quantities.

The foregoing derivation of $\Gamma^{(1,1)}$ and $\Gamma^{(2,2)}$ is easily extended to still higher order quantities. Any of the correlations defined by Eq. (II.34) can be obtained in the same manner. 
III. SIGNAL, NOISE, AND LIGHT INTENSITY

A. Quantum Optics and Intensity Correlations

The analyses in the preceding chapters have all been $d$ one vithin a framework of purely classical optics. But, of course, a classical theory is merely a limiting expression of the more furdemental quantum optics. The present chepter takes up the question of estimating the ratio of signal to noise in the output of a wultiple-ieam spertrometer. In this analysis, ihe discreteness of light quanta is important.

Such discussions usurliy assume that photon detection is a random process whose probability density is proportional to the classical light intensity. This assumption received some attention at the time of the first intensity correlation measurements, whtch were done by Hanbury Brown and Twiss. ${ }^{44}$ in their analysis of thet experiment, ${ }^{45}$ Hanbury Brown and Twiss used both classical and quantum theories. They interpreted the agreement of the classical and quantum calculations as an expression of the principle of complementarity: one can interpret the interference process in terms of waves and then explain the detection process in terms of discrete particles. Since interference involves the weve aspect of ligit, a classical analysis is vald for that pert of the experiment. The same experifuent was again analyzed by purcell, ${ }^{46}$ who elso equated classical intensity witn photon probability. In both of these papers, the point was made that one can elso analyze the interference prncess while discussing photcns, but that a wavi picture is uore conventent because a light wave 
may be treated as a classical fleld, while photons cannot be treated as clessical particles.

It perhaps should be emphesized that the multiple-tean spectrometers which we are considering here are not intensity correlation devices. The spectrometers discussed in rhapter II select two observed waves, $A$ and $B$, which are combined and then reseparated into interference patterns 1 and 2, giving information about the phase of $A$ and $B$. ro make an intensity correlation measurement, on the other hand, one would not combine beams $A$ and $B$ at all. One would simply measure the Intensity of A with one shototube and the intensity of $B$ with another phototube and then record tine correlation of the outputs, This is the technique which was first demonstrated by Hanbury Brown and Tw1ss. It provides a different way of getting information about the mutual coherence of two light waves. Intensity correlation measurements have replaced the other mathods in certain applications, but the multiple-beam spectrometers which ie are consiajering belong to the older, standard class of interferometric instrument:s.

But this is not to say that an intensity correlation measurement could not be useful in the observation of a plasma. Indeed, this technique ahould be important there for the same reasons for which 1t was developea in the first place. Intensity correlation wethode were first developed for use in stellar interfercistry. It had long been known that one could use an interferometer to measure th's apparent diameter of a star, but the utility of this technique was limited by the effect of atmospheric scintiliation. 
Veriations in the Earth's atmosphere cause varietions in the phese of starlight, and at points too widely separated these varlations are uncorrelated, destroying the effect of interference. This IIfft.ts the useable beseline, and hence the resolution of a conventional stellar interferometer. But an intensity interferomet.er is largely unaffected by this difficulty. The intensity is a much more slcrly changing quantity than the amplitude, and changes in path of many wavelengths can be tolerated.

In a plasma, variations in the index of refraction could produce the same effect. If the index of refraction were not exactly unity, differences in $n$ along the different lines of sight could cause phase changes and destroy the interference. ${ }^{47}$ As in observations of a star, the use of an intensity interferometer would permit extension of the method to sucis cases.

Since the first intensity correlation measurements, the Invention of the laser and iaprovements in other optical devices have led to renewed intersst in the quantun theory of optics. The present state of this subject is described in several recent books. ${ }^{2}$

The interpretation of photodetection data, and the relation between classical and quantum optics--both topics of perticular importance for our discusaton iere-are sonsidered in detail in one paper by Glauber. ${ }^{1} 8$ Strictly speaking, only radiation flelds containing many quant 1 are described by ciassical theory, and, moreover, there are many quantum states which even with large quantum numbers do not seem to correspond to any classical descrip- 
tion. Noting this, Glauber then describes a certain cless of states (states whose density operators can be written in a form which he calls a "P representation", with a positiv/weight function) whose averaged behavior--in particuler, thes.. quantum mechanlial correlation functions--may be written in a form stmilar to classical theory, even in the limit where the occupation numwers of the states are very smal" The light euritted by chao:ic sources, he observes, is always of this form.

The same peper also anelyzes the significance of photon counting measurements. Usins: a simple mudel of a photon counier, Glauber obtains an expressio.1 for the factorial moments of the photocount probabllity distribution in terms of the correlation functions of the radiation field. [His Eq. (51).] This result Is much more general than any which we will be using here, but for the first moment it just gives the mean number of counts exzected in a given interval. This mean is proportional to the time integral of the first correlation function, i.e., to the quartity which corresponds to slassical intensity. For flelds wisch correspond to clessical descriptions, Glauber then goes on to obtain an expression for the complete photocount probability distribution. This he shows to be an integral over foisson distributions, a reault which cen aj.so be obtained from semiclassical analysis. (See Fef. 49.)

The quantum theory, of course, provides much more than fust a basis for a clecisical description. But for our problem here, a full quantum trestment is not really needer, so long as one 
accepts the two assumptions that a clessical analysis describes the interference of the light and that the classical intensity may then be taken as a photon probability in analyzing the detection process. One can then explain both interference and photon statistics. We will not discuss the basis of this simple (and quite standerd) picture any further here. More complete explanations of the link between this semiclassical description and the full quantum theory can be found in the paper by Glauber just cutlined $^{48}$ and in the books on ouantum opties mentioned earlier. ${ }^{2}$ B. Classical Noise

Even if a completely classical description of the light were adequate, a multiple-beam spectrometer, like any other instrument, would generate some noise. Before beginning an analysis of photon statistics, one should obtain at least an estimate of the amount of nolse to be expected from such other sources as stray light, mechenical vibrations, and fluctuations in the electi onics. Ideally, in the classicel picture, the light in either beem A or bear $B$ alone should contribute nothing to the output signal. Such light should be divided equally between beams 1 and 2 , addIng nothing to their intesoity difference.

It perhaps should be emphasized that on: actually could balance out the separate effects of both of the beams $A$ and $B$. one could obviously zero elther one of them by adjusting the phototube gains, but the same setting which belanced A would probebly not be exactly right for B. There is, however, a second possible adjustment. One could also rotate slightly the polariza- 
tions of $A$ and $B$ with respect to $I$ and 2. Th1s would put more of $A$ into $I$ and sore of $B$ into 2 or vice versa. By using this "differential" control, together with the "common" callbration of the phototubes, one could, in princlple, precisely rull out the effect of each of the two beams.

In reality, this belencing would not be perfect, but the effect of noise could then be minimized by signal processing. In many observations, the spectrum $Y(\omega)$ of the signal $Y(t)$ would have sharp maxima at certain frequencies. For example, if the observed source density component were produced by a plasma wave of frequency $\omega_{0}$, this would appear in the recorded signal spectrum. The noise, on the other hand, should be spread out over some wider band of frequencles. (Remember that the signal comes from only one component of the source distribution. Thus the signal spectrum would usually be much narrower than the whole spectrum of plasma disturbences. Thus even if the noise came from the plasma, it would still be spread over a wider band.) So there are, so to speak, two "Iines of defense" against unwented background light. F1rst, one can null out beams $A$ and B alone. Ideally, this should eliminate everything except the slgnal. Then any nolse which does get through (or which is generated later in the system) can be removed in an analysis of the time or frequency defendence of the signal. Roughly estimating, it should be possible to balance the phototubes to within one percent. Then if the signal spectrum is at all unique, one should be able to do at least as well again in the signal spectrur 
analys1B. Together, this would give discrimination of one part In $10^{4}$, and probably several orders of magnitude more.

Furthermore, if it were possible to modulate the observed phenomenon, one could use phese sensitive detection, which would give a large additional improvement. Another way to use this aame technique, if the phenomenon were stationary, would be to modulate the light with a toothed wheel or other shutter and then phase lock onto that signal. Thls would not discriminate against the background light, but it would stop nolse generated later in tine system (e.g., in the amplffier or the spectrum analyzer). one cannot say much more in generel. The sensitivity is any given measurement would depend upon the apparatus used orut upon the spectral properties of the selected signel. But it does not appear that "classical" noise would present any mejor problem. It seems evident that in almost any situation in which a multiple beam spectrometer could be used, the observation woulc be limited by photon statistics, rather than by other types of nolse.

\section{c. In Estimate of Photon Stailistice}

For an analysis of photon statistics, it is convenient to express each light intensity in terms of a mean counting rate. our system measures two 1ntensities, $I_{1}(t)$ and $I_{2}(t)$, and these In turn consist of two distinct components: "beckground light" and "oignal 1f.ght". The beckground light is that which classicaljy is juat divided equally between beams $I$ and $z(e . g .$, Ifeiht frcm a source observed only through beam A. Th1s is not to be confused with "stray light" which can be reduced by optical im- 
provements). The signal light is that emitted by the one observed component of the light source distribution $\left[T\left(\underline{r}^{\prime}, \omega\right)\right.$ in the terminology of section II.B.2]. Describing both of these in terms of photon flux, we have

$2 Q$ beckground photons/second

P signal photons/second.

$2 Q$ is divided equally between phototubes 1 and $2 ; P$ is unequally divided and varles between the two in a manner determined by the time dependence of the observed source density component. These two intensities, whose values were obtained from classical enalysis are now to be treated as photon detection probabilities. For an ideal photon counter, this description can be justifled by semiclessical analysis, as explained in the preceding section.

A photomultiplier tube is rot a perfect photon counter, lut for any such devices, a similar description of the output is appropriate. This can be seen from an investigation of photomultiplier properties reported recently by Robben. ${ }^{50}$ After measuring the pulse charge spectrum and the fluctuations in the outputs of a variety of different photomultiplier tubes, Robben was able to describe the nolse propertles of each tube in terms of three perameters: the overall quantum efficlence, $\eta F$; a photoelectron nolse factor, $S$; and an effective dark rate, $D$. The quantum efficiency $\eta$ and photolectron collection $F$ were used as uavally defined. The factor $\mathrm{S}$ was defined in terms of excess measured noise, but in the simple model of a phototube producing 
pulses with a range of amplitudes, followed by a discriminator which accepts all those above some threshold, $S$ is just the inverse of the fraction of the total palses which are counted. D is the measured dark rate at the same discriminator setting. These quantities were found to be independent of frequency, except for perlods longer than about 10 seconds. Thus, for many observations, $P$ and $Q$, the signal and background contributions to the counting rates could just be modified to take into account the fractional detection and the dark rate:

$$
\begin{aligned}
& P^{\prime}=\frac{\eta F}{S} P \\
& Q^{\prime}=\frac{\eta F}{S} Q+D .
\end{aligned}
$$

So in the following analysis, the measured light intensities, whlch will be speclfled in terms of $P$ and $Q$ may be interpreted as photon fluxes (measured by a perfect photon counter) or, more realistically, as photomultiplier output pulse rates, given by $P^{\prime}$ and $Q^{\prime}$ in Eqs. (III. Ia, b).

If the signal were at zero frequency, the photon statistics would be simple, Assuming that $P$ went entirely into beam 2 , one would have

$$
\begin{aligned}
& I_{1}=Q \\
& I_{2}=Q+P .
\end{aligned}
$$

Provided that $Q \gg P$ (which is assumed throughout the following), the signal to nof se ratio would be 


$$
\frac{\mathrm{S}}{\mathrm{N}}=\left[\frac{(\mathrm{PT})^{2}}{2 Q T}\right]^{1 / 2}
$$

where $T$ is the total time of observation.

In general, the problem is more complicated than th1s, since the signal $Y(t)$ is time dependent. In such s.tuations one would be likely to record a signal spectrum $Y(\omega)$. To analyze this operation, consider now a case in which the observed component of the lifht source density is all due to a plasma wave of frequency $\omega_{0}$. Then $P$, the signal light, would oscillate between the phototubes at this rate and the spectrum would show a peak et $\omega_{0} \cdot$

We represent a spectrum analyzer by the model shown in Fig. III-1. In such a device the signal $Y(t)$ is first mixed with the output of a reference oscillator and the result is then averaged over a time $\tau$. Since the mixing heterodynes the signel down in frequency by an amount equal to the frequency of the reference oscillator, and since the $\tau$ average transmits only those frequenctes below $1 / \tau$, the result represents the signal frequency components in a band of width $1 / \tau$ around the reference frequency. To measure the amplitude of the signal within this band, the output of the $\tau$ average, $Y^{\prime}(t)$ is squared, giving a signal $z(t)$. Th1s quantity is then everaged over tive observation time, which we again denote by $\mathrm{T}$.

Thus the observation of a signal spectrum involves two time Intervals, an inverse bandwidth, $\tau$, and $T$, the total time of observation at one signal frequency. (If the reference frequency 


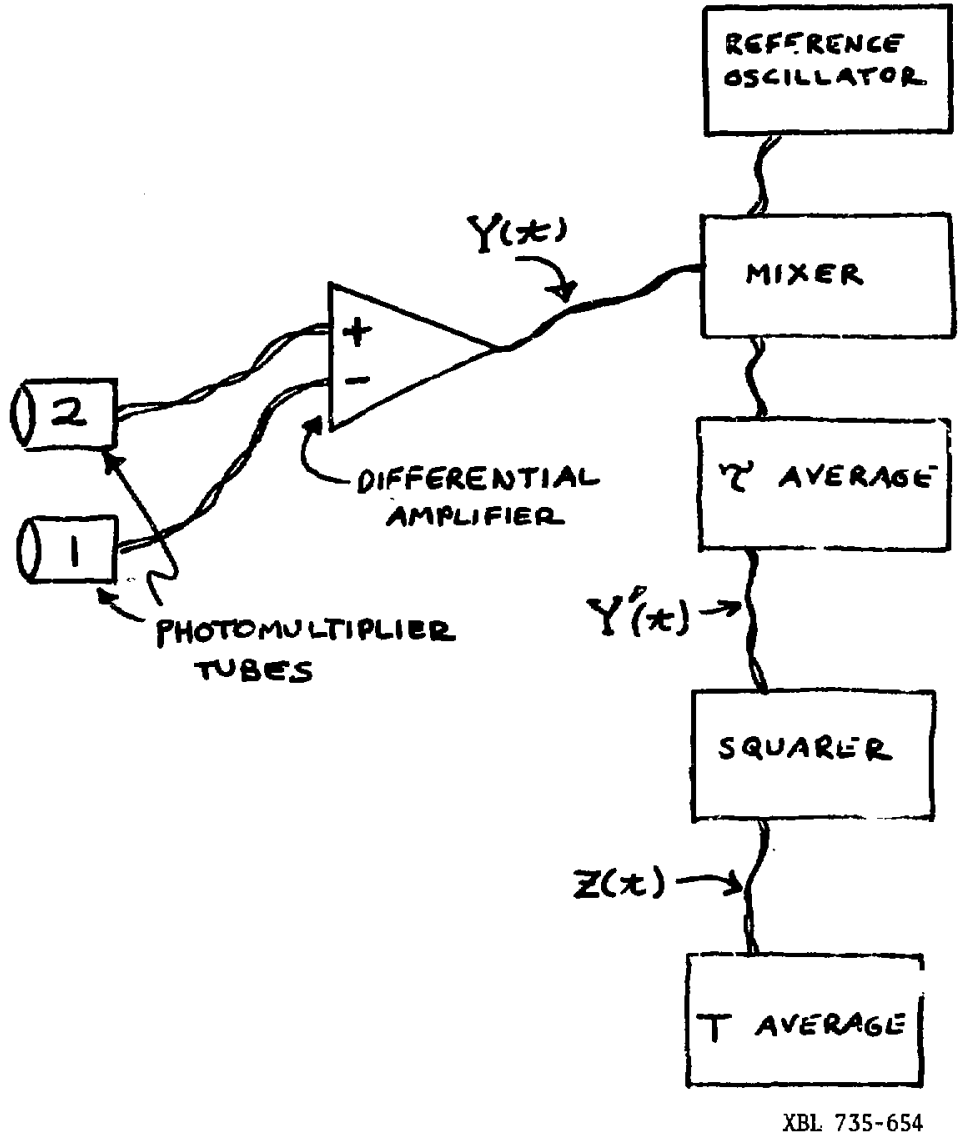

FIg. III-1. A model of the electronic apparatus. 
is swept, T is the time needed to sweep across one bandwitil.) since all tmansmitted frequency components lie within $1 / \tau$ of the reference, their phase relative to the reference caniot chanse within a $\tau$ interval. In terms of counting statistics, the signe.l probability is roughly constant over any $\tau$ interval, so for such times the problem is effectively at zero frequency. On the other hand, at times differing by much more than $\tau, Y^{\prime}(t)$ is an verage of different inputs, so such velues represent completely separate samples of the signal spectrum.

To describe these two extrenes, it is convenient to divide the observation time into a set of $\mathrm{T} / \tau$ discrete $\tau$ intervals. As an approximation, one can then consider each $t$ interval separately, sssuming that $z(t)$ at the end of eacs depends only upxn signals received during that same interral, and also that all such inputs may be welghted equally. Since the se are then $c$ smpletely Independent samples, the signal-to-nolse ratio expected in the $T$ average is

$$
\left(\frac{\mathrm{S}}{\mathrm{N}}\right)_{\mathrm{T}}=\sqrt{\frac{\mathrm{T}}{\tau}}\left(\frac{\mathrm{S}}{\mathrm{N}}\right)_{\tau}
$$

where $(S / N)_{\tau}$ is that expected from the counting distribution dus to $z(t)$. In figuring these quantities, we shall consider all counts equal in magnitude, but since the phototube signis are differences, and also multiplied by a reference signal which takes elther slcn, one must include butn positive and nezaìive pulses. If $\mathbf{n}$ is the recorded elgebratc sum of counts, one has for a $\tau$ intervaI 


$$
\left|\frac{s}{N}\right|_{i \tau}^{2}=\frac{\left[\begin{array}{l}
\langle n\rangle_{z \text { with }}-\langle n\rangle_{z \text { withnut }} \\
\text { signal }
\end{array}\right]^{2}}{\left(n^{2}\right\rangle_{z}-\langle n\rangle_{z}{ }^{2}} .
$$

Here () denotes an average and the subscript $\mathrm{z}$ refers to the assactated probability distribution. As indicated, the "signal" is not the spectral amplitude; it is the difference between the amplitude with signal (1.e., with the reference frequency $\approx \omega_{0}$ ) and that without signel (or with the reference osclilator at another frequincy). In elther case there will be some spectral anplitude due to the randemly distrlbuted background counts. To be observalle, the effect of lie signal must exceed the fluctuations in the measurea background spectral amplitude.

$$
\text { since } \begin{aligned}
Z(t) & =Y^{\prime}(t)^{2}, \\
\langle n\rangle_{Z} & =\left(n^{2}\right)_{Y^{\prime}} \\
\left\langle n^{2}\right\rangle_{Z} & =\left\langle n^{4}\right\rangle_{Y^{\prime}} .
\end{aligned}
$$

$Y^{\prime}$ in turn is equal to the difference between $Y_{2}(t)$ and $Y_{1}(t)$, the positive and regative components of the output of the filter. ( Jn a zero frequency observation, these would be fust $I_{1}$ and $I_{2}$. In an observation at a higher frequency, the multiplication by a reference ascillation switches $I_{1}$ and $I_{2}$ between $Y_{1}$ and $Y_{2}$ at the reference frequency.) Over a $\tau$ Interval, the signal $P$ is divided unequally between $Y_{1}$ and $Y_{2}$. By definition,

$$
Y^{\prime}(t)=\int_{t=\tau}^{t} d t^{\prime}\left[Y_{2}(t)-Y_{1}(t)\right] \text {. }
$$


Hence the counting distributions are related by an integral

$$
P_{Y^{\prime}}(n)=\int d u^{\prime} P_{Y_{1}}\left(n^{\prime}\right) P_{Y_{2}}\left(n+n^{\prime}\right) \text {. }
$$

Fram this it follows that,

$$
\left\langle n^{2}\right\rangle_{Y^{+}}=\left\langle n^{2}\right\rangle_{Y_{2}} \cdot 2\langle n\rangle_{Y_{1}}\langle n\rangle_{Y_{2}}+\left\langle n^{2}\right\rangle_{Y_{1}}
$$

and that

$$
\begin{gathered}
\left\langle n^{4}\right\rangle_{Y^{\prime}}=\left\langle n^{4}\right\rangle_{Y_{2}}-4\left\langle n^{3}\right\rangle_{Y_{2}}\langle n\rangle_{Y_{1}}+6\left\langle n^{2}\right\rangle_{Y_{2}}\left\langle n^{2}\right\rangle_{Y_{1}} \\
-4\langle n\rangle_{Y_{2}}\left\langle n^{3}\right\rangle_{Y_{1}}+\left\langle n^{4}\right\rangle_{Y_{1}} .
\end{gathered}
$$

Finally, the distributions for $Y_{1}(t)$ and $Y_{2}(t)$ we take to be Polssonian. If the signal is all contained in $Y_{2}(t)$ this gives,

$$
\begin{aligned}
& P_{Y_{1}}(n)=\frac{(Q \tau)^{n}}{n !} e^{-Q \tau} \\
& P_{Y_{2}}(n)=\frac{[(Q+P) \tau] n}{n !} e^{-(Q+P) \tau}
\end{aligned}
$$

This is an approximation too, since it 1gnores the rapid fluctuations in the classical intensity, but since the times consldered here are all much longer than the coherence time of the light, the neglected increase in $\left\langle\mathrm{n}^{2}\right\rangle$ and other moments, the "excess photon noise", is relatively small. (See Ref. 49.)

All the needed moments can be calculated from these distributions. Using EqG. (III. 5a,b) to write the moments of $\mathrm{Y}^{\prime}$, and keeping only leading terms, one obtains 


$$
\begin{aligned}
& \left\langle n^{2}\right\rangle_{Y^{\prime}} \underset{\substack{\text { with } \\
\text { signal }}}{ }-\left\langle n^{2}\right\rangle_{Y^{\prime}} \underset{\substack{\text { without } \\
\text { signal }}}{ }=(P \tau)^{2} \\
& \left\langle n^{4}\right\rangle_{Y^{\prime}}-\left\langle n^{2}\right\rangle_{Y^{\prime}}=8(Q \tau)^{2} .
\end{aligned}
$$

From Eqs. (III.3) and (III.4a,b) one can then calculate the sibnal-tonnotse ratio for the $\tau$ average,

$$
\left(\frac{S}{N}\right)_{\tau}^{2}=\frac{(P \tau)^{4}}{8(Q \tau)^{2}} .
$$

Then from Eq. (III.2) one obtains the signal-to-noise ratio for the whole measurement

$$
\left(\frac{\mathrm{S}}{\mathrm{N}}\right)_{\mathrm{T}}^{2}=\mathrm{T} \tau \frac{\mathrm{P}^{4}}{8 \mathrm{Q}^{2}} .
$$

So, for the effect to be observable, one must have

$$
P \sqrt{T^{T}}>\sqrt{/ B} \frac{Q}{P}
$$

Here the numerical factor should not be taken serlously, since several simplifying approximations heve been used, but the result show the deperdence on the different factors and indicates when such an observation should be feastble. 


\section{EXPERTMENTAL RESULTS}

\section{A. Tests is' the optical System}

\section{The Calcite Rhomb}

To test the conclusions of the foregoing anilysis, several multiple-venm spectrameters were assembled and studied, A central element in all of the arrangements used was a calcite rhomb, $1 \mathrm{~cm}^{2}$ in aperture, whick is shown in Fig. IV-l.

A.s a first step, the optical properties of the rhomb alone were examined, using light from a small He-Ne laser. The laser was equipped with a beam-expanding telescope, which when focused at. Infinity produced a beam $2 \mathrm{~cm}$ in diameter--large enough to illuminate the whole face of the rhomb. To observe the effect of the rhomb, a ground glass screen was placed approximately I m behind the rhomb and a camera behind the screen was used to photograph the 1llumination patterns.

The laser light was inttielly linearly polarized st $45^{\circ}$ to the principal axes of the ruomb. A second linear polarizer was placed before the ground g'ass screen, to permit observation of different polarization components of the finel pattern.

Altogether, the ordering of elements was:

leser,

bear-expanding telescope,

linear polarizer (at $45^{\circ}$ to the axes of the rhamb), celc1te rhomb,

Inear polertzer (rarled to display the different patterns), ground glasi screen, 


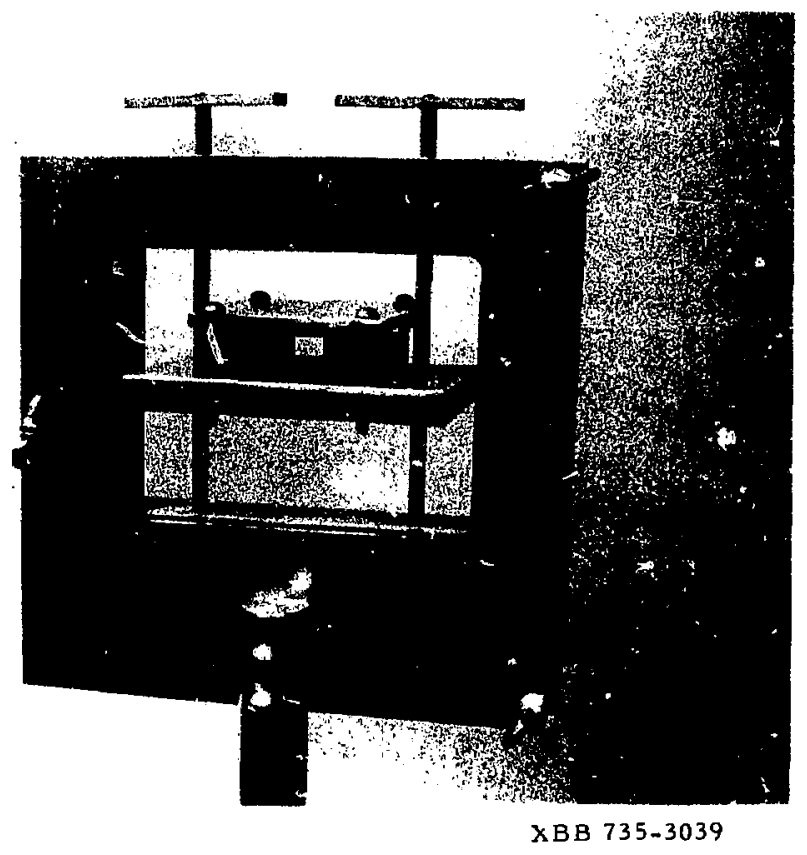

Fig. IV-l. Calcite rhomb in mount. 
cemera, focused on the screen.

Thus this arrangement was like that shown in Fig. C-2 of Appendix C, except that in the present case the telescope was defocused to give a slightly expending beam, a mask was not used, so the whole rhomb was illuminated, and the lens in Fig. C-2 was not nee led, since the beams already overlapped.

The incident laser light was divided by the rhomb into two equally intense, orthogonally polarized components-- " $A$ " and " $B$ " in the terminology of Chapter II. When the polarizer before the glass screen was orfented to select efther of these components, a Emooth distribution of intensity resulted, as 1s shown in the photographs in F1g. IV-2. But the intermediate component, "1" revealed a set of distinct interference fringes, and the orthoganal component "2" displayed the opposite or complementary set of fringes, as is shown in rig. IV-3.

The ae are polarization fringe potterns, produced by interference between the $A$ and $B$ components of the light. Since $A$ and B were orthogenally polarized, the total light intensity (also shown in F1g. IV-2) was just the sum of the intensities of $A$ and $B$. But since $A$ and $B$ were coherent, they conld interfere to give a pettern of varying polarization.

To d1splay such results more concisely, one ca:ı place two orthogonally orlented polarizers side by side, so that each covers half the pattern. Then both the A and $B$ components, or both the 1 and 2 components of the light can be observed. Such results are shown In FIg. IV-4. There the left frame shows the A and B com- 

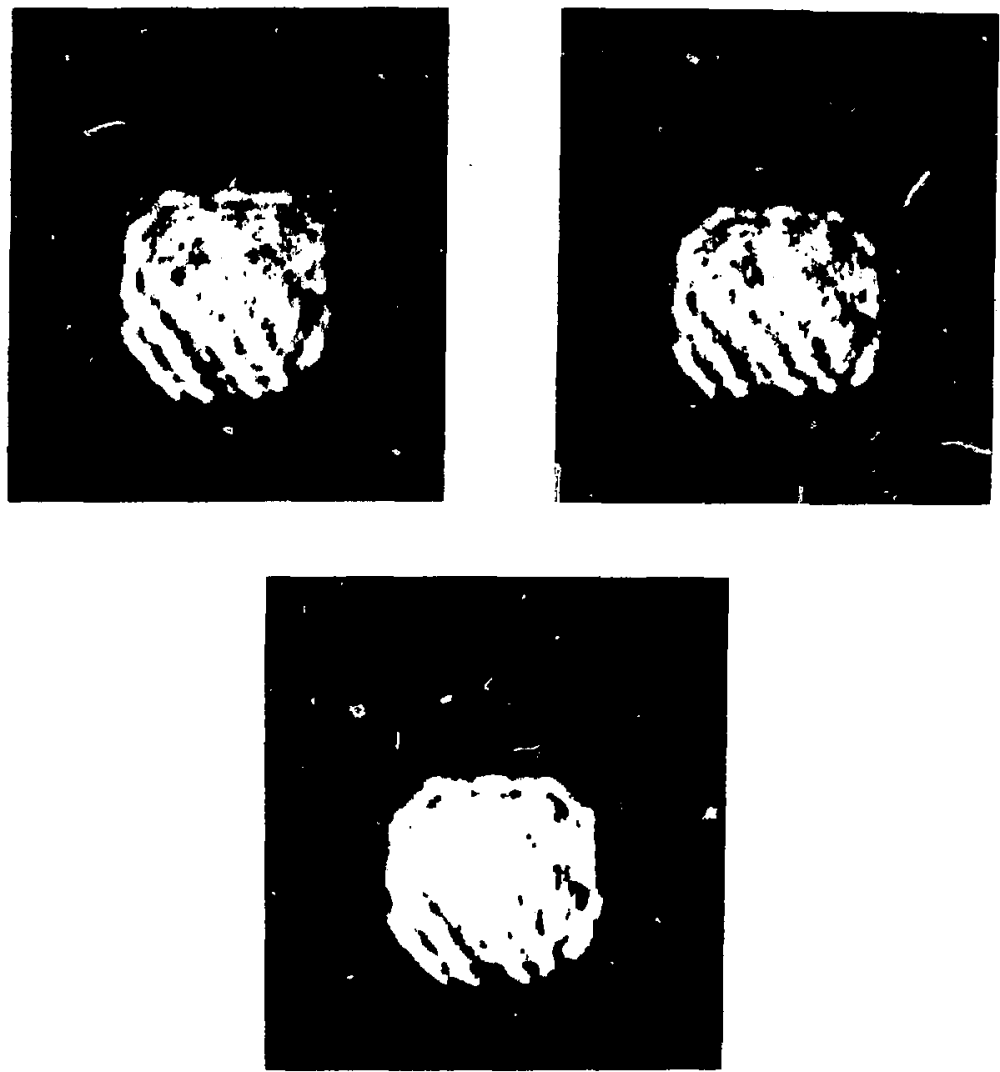

ХВВ $733-2372$

Fjg. IV-2. Illumlnation patterns with the rhomb alone.

Top left: polarization A; top right: polarization $B$; bottam: total light intensity. 

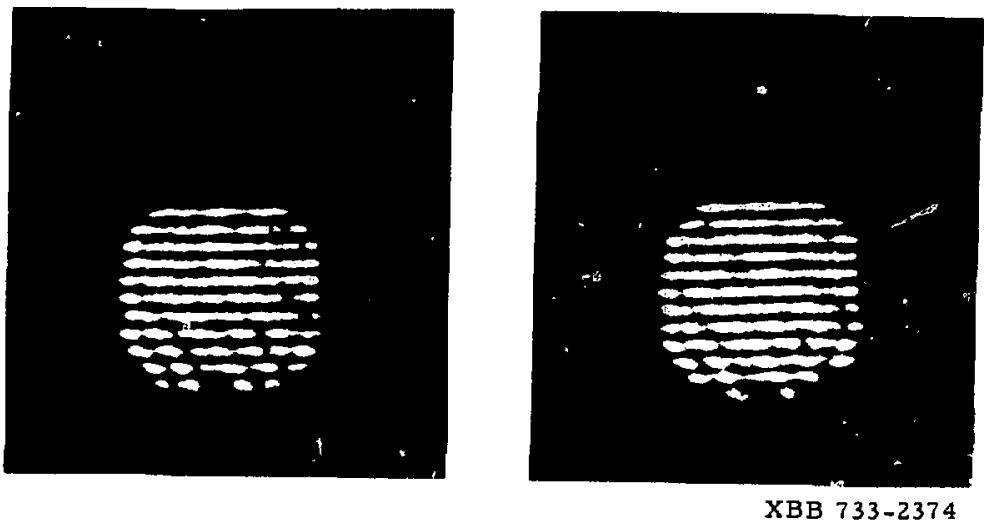

Fig. TV-3. Illumination patterns with the rhomb alone. Left: polarization 1; rieht: polarization 2 . 

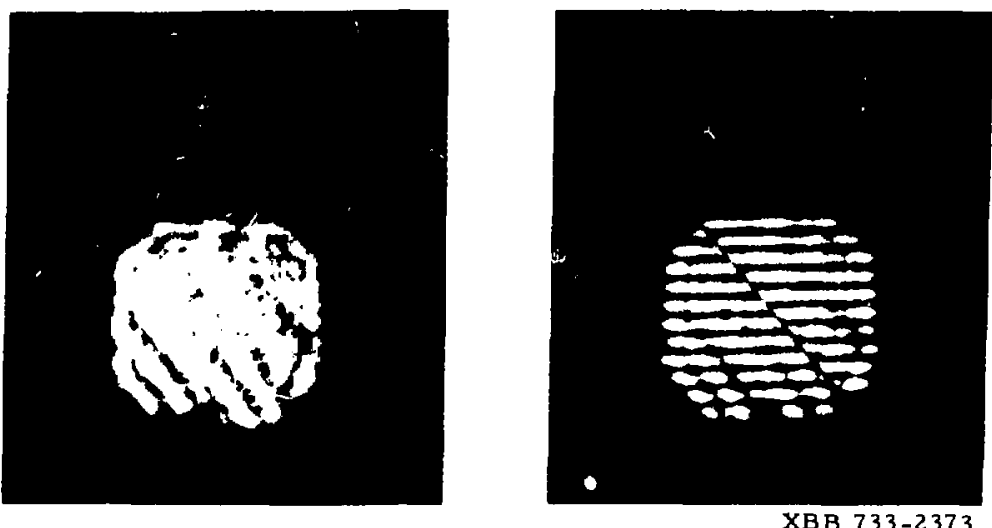

F1E. IV-4. Illimination patterns with the rhomb elone.

Left: polarizations $A$ and $B$; right: polarizations $I$ and 2. 
ponents (the left and right halves of the pattern) and the right frame shows components $I$ and 2 . Here it is particularly evident that patterni 1 and 2 are complementary.

'These results are illustrations of the kind of polarization interference effects which we propose to use to measure correlations in the light emitted by plasma. Moreover, since all of patterns in these figures were made while illuminatiug the entire rhomb, the sherpness of the fringes served to demonstrate that this rhomb was of sufficient quality for use in such a system.

\section{A Two-Beam Spectroscopic System}

As the next step, an elementary two-beam spectroscopic system was assembled. In this arrangement, the calcite rhomb was used in series with a monochromator. A mask behind the rhomb served to define the twn observed beams, a lens before the rhomb defined a common source volume, and finally a second lens locused the Ifght onto the entrance slit of the monochromator, exactly as illustrated in Fig. C-4 of Appendix C.

If this system had been used to observe a plasma, a Inear polarizer would have been placed behind the first lens, causing the trangmitted light to be divided into equal A and B components by the rhomb. These components would heve been recombined at the monochromator entrance and the light which was transmitted by the monochromator would have been separated Into 1 and 2 components by a prism placed behind the exit slit.

However, for testing the system it is much more convenient to interchenge the roles of source and observation point. If the 
exit slit of the monochromator is llluminated with light polarized as 2, that 11 ght w1ll retrace the optical path in reverse, w11l be divtded Into $A$ and $B$ components by the rhomb and these components will be recombined at the former location of the common source volume. There all those points for which the path lengths of the $A$ and $B$ components differ by an integral number of wavelengthe will be 11 luminated by light polarized as 2 , while all the points for which the two path lengths differ ly a half integral number of wavelengths will recelve light polarized as 1. Since none of the path lengths is changed by reversing the direction of the light, these points are exactly the locations from which sources would, in the original arrengement, have contributed light of the same 1 or 2 polarization to the output of the system. Thus, in this way, one cen obtain directly a display of the observed component of the light bource distribution.

Th1s procedure was used to test a varlety of systems. For the two-beam system, the arrangement was:

He-Ne laser,

If near polarizer (uith the orientation " 2 "),

lens, focused on the exit slit of the monochromator, monochromator,

lens, focused on the entrance slit of the monochromator, mask with one slit, calcite rhomb, lens, focused on tis wereen, two orthogonal linear polarizers side by side (varled 
to display the different patterns),

ground gless screen,

camere, focused on the screen.

In normal operation, all of the light transmitted by the monochrometor would contribute to the slgnal. Fence in the present reversed operation, the entire exit slit should be 1lluminated. This was accomplished by sweeping the focus of the laser beam along the exit slit during exposure of each photograph. Sweeping the focus in effect expands the laser beam, but in a way which prevents interference between light from different points aloug the slit.

The 1llumination patterns which this system gave are shown 1n FIg. TV-5. There the upper pettern shows polarizations 1 and 2, while the lower pattern shows polarlations A and B. Again, the A and B components produced smooth patterns (the faint vertical bands are due to unsteadiness in sweeping the beam), but the 1 and 2 components gave sets of sharp interference fringes. As just explained, the patterns 1 and 2 in FIg. IV-5 show the locetione from which sources would contribute light of that polarization to the output when the system was operated normally. The final measured quantity in normal operation $1 \mathrm{~s}$, of course, the difference, $Y(t)=I_{2}(t)-I_{1}(t)$, between the intensities of the se two components of the output 11ght. Hence, in FIB. IV-5 it 1s the difference between fringe patterns 1 and 2 which corresponds to the component of the density of light sources which would be measured by this system if it were used to observe $a$ 
$-112-$

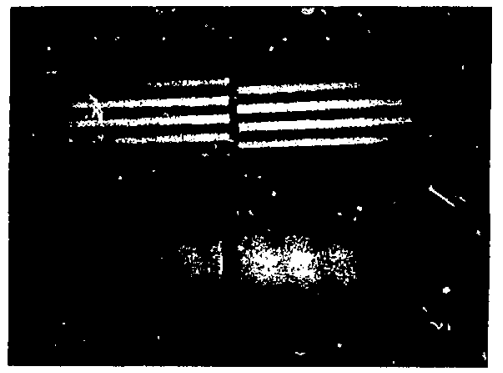

XBB $734-2425$

Fiv. IV-5. The effect of a two-beam spectrometer. Top: polarizations 1 and 2; bottom: polarizations $A$ arid $B$. 
plasma. It is clear from the petterns that inis difference is just one wavelength or $\underline{k}$ component of the distribution of Iight sources near the focus of the system. Thus these photographs ogree with the predictions of the theory, with the result described in Sect. II.A by Eqs. (II.13) and (II.15) and described in sect. II.B by Eq. (II.29).

The theory also predicts that the signal from a two-beam spectrometer will be due exclusively to sources within a restricted "common source volume". Indeed, the achievement of spatial localization was the first objective of the whole project. Thus, ilthough the result is fairly cercain, it is still important to check the effect of thls system eway from focus. This was done by moving the ground glass screen and the canere closer to the otiner optical components. (The distance between the screen and the last lens was roughly halved.)

The result is shown in Fig. IV-6. There the top frame shows agein some patterns taken at the focus, while the lower frame shows the results away from focus. In the lower frame beams A and $B$ are clearly seperated, and patterns $I$ and 2 , which now do not exhibit fringes, are seen to be 1dentical. Hence, the intensity difference signal, $Y(t)$, will always vanish for sources this far from the focus. This reglon makes no contrikution to the signel, irrespective of the distributicil of the sources there. Thus the result of a two-beam spectroscopic observation would not be an average along a line of sight. Sources this far from the focus would not be observed. 

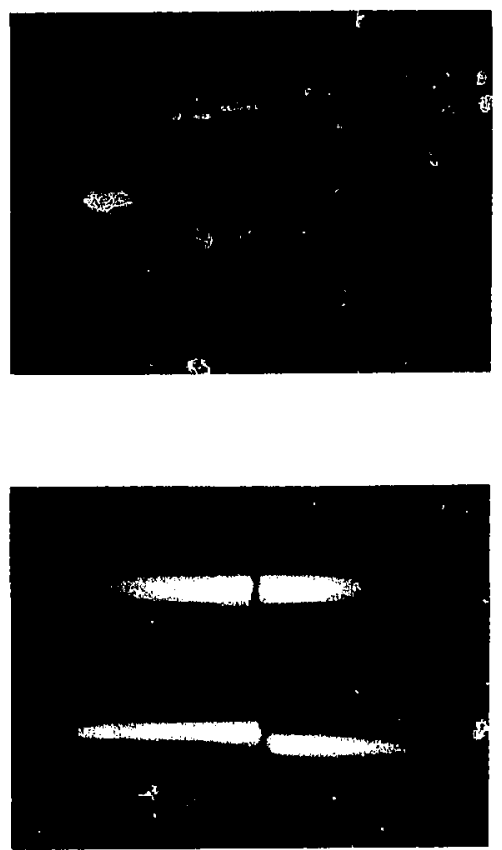

XBB $734-2424$

FIg. IV-6. Spatial resolution with a two-beam system. Top frame--patterns in the focal plane of the system (upjer pattern: polar1zations 1 and 2; lower pattern: polarizations $A$ and $B$ ).

Bottom frame--patterns at a distance from the focus

(upper pattern: polarizations 1 and 2; lower pattern: polarlaations $A$ and $B$ ). 
Although one can thus make a local measurement, our theory predicted that--at leest with a two-beam system--one can observe only fluctuations in the light source density. One cannot observe the total nunber of light sources in some reglon, essentially because the wavelength of the observed source density component $\left(2 \pi /\left.\right|_{\Delta} \mid\right)$ is necessarily emaller than the width of the focal region. This is true because the former varles inversely with the angle between beams $A$ and $B$, while the latter varies inversely with the angle subtended by efther beam alone (angles $\alpha$ and $B$, respectirely, in Fig. (-4). This conclusion was also checked experimentally, and the results are shown in Fig. IV-7. In our apparatus, the angle between the beams was determined by the focal lengths of the lenses and by the lateral displacement of one beam by the rhomb. Th1s wes left unchanged. The angle subtended by each beam, however, was determined by the width of the slit in the mask before the rhomb, and this was varied to produce the three sets of patterns shown in Fig. IV-7 (again taken at focus). The upper pattern was made $w 1$ th the largest slit, the center pattern with a rarrower slit, and the lower pattern with a still narrower slit. Thus the angle $B$ subtended by each beam was progressively decreased, and, as expected, the number of fringes in the pattern is seen to vary inversely with this engle.

Beyond a simple confirmation of the theory, the Importince of these results is that they showed that an optical system of the type envisioned could be made from components of quite ordinary quality. The width of the beams A and B covered a substantial 

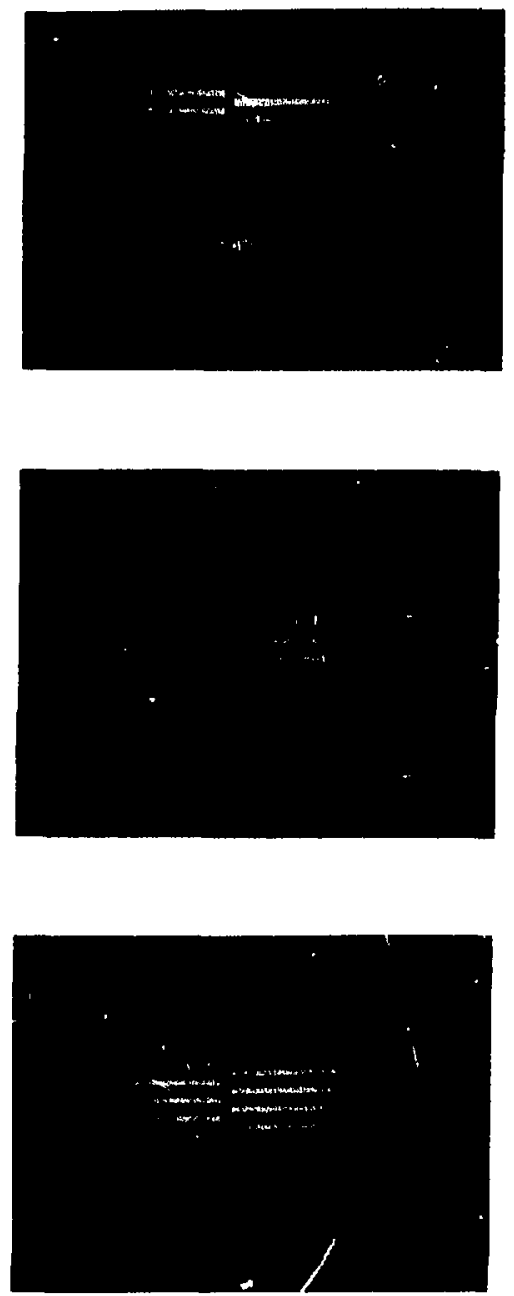

XBB $734-2433$

Fig. IV-7. The effect of the width of the beam-defining slit. Top freme: widest slit; botton frame: narrowest slit. 
portion of the diameter of the lenses used (and in the multiplebeam systems described below; the whole set of beams covered 5 large part of the lens area), yet the Interference patterns obtained were sharp and clear. This occurred because the displacement due to the rhomb was fairly small and thus the interfering components of different beams passed through adjacent portions of the lens. It is only necessary that the different interference patterns coincide, but that is in essence a requirement on the imaging quality of the lens: So long as the lens is good enough to image a point to a spot mich amaller than the desired fringe spacing, then the interference patterns produced by pairs of beams which go through different portions of the lens will coincide and the whole pattern wili be sharp and clear.

I want to emphasize this point. At no time in this experimental work was any difficulty due to poor lens quality encountered. The produc uion of polarization interference fringe patterns does not require "interference quality" components. Our systems were made with lenses already in the laboratory and they always produced fringe patterns like those shown in the photographs presented here.

\section{Some Multiple-Beam Systems}

Thus the two-beam system performed as expected. This apparatus could be used to observe fluctuations in a plasma, but such an observation would be difficult because the two-beam system is so Inefficlent in its use of the available light. It was for this reason that some more complicated systems were designed (as 
described in Appendix C) and analyzed (as described in Sect. II.B). In the experimental work, several multiple-bear systems were also constructed and tested.

To simplify these observations, only the interferometric portion of these systems was constructed. The results of the work w1th the two-beam system proved that tae inclusion of the monochromator did not degrade the polarization fringe patterns. In those studies, however, the first part of the optical train,

leser

lens, focused on the exfit slit of the monochrometor monochromator

lens, focused on the entrance slit of the monochromator, simply served to produce a beam of parallel monochromatic light. It was important to establish that this could be done with the required accuracy, but once that had been demonstrated, this part of the system could be replaced by just the laser and a beamexpanding telescope.

To make a multiple-beam system of the first type considered 1n Appendix C, one need only replace the mask behind the rhomb with one containing many slits. This was done in the simplified system:

He-Ne laser,

Deam-expanding telescope,

Iinear polarizer (orlented at $45^{\circ}$ to the axes of the rhomb),

mask with slits to define the beams, celclte rhomb, 
lens, focused on the screen (except when the patterns were observed away from focus),

linear polarizer (varied to display the different patterns),

ground glass secreen, camera, focused on the scjeen.

In tests of the two-beam system, the focus of the l1ght was swept along the exit slit of the spectrometer. This zeused an image to sweep along the entrance slit, and the lens focused there produced a collimated beam swept in direction. Thus the effect of illuminating the entire slit could be simulated in the present simplified system by rotating the laser and telescope during exposure of the photographs. However, the work with the two-beam system showed that th1s would only spread the patterns horizontally. Since all the features of Interest can be seen without such a spreeding, the multiple-beam patterns studied next were simply photographed at one position of the laser beam. 'The results thus show a single vertical slice of the complete fringe patterns. The dealgn of a mult1ple-beam system actually began when it was realized that the effect of a simple two-beam system should be independent of the exact position of the silt which defines the beams. In Appendix $C$ it was argued that a cinange in the position of the alit in the mask behind the rhomb would not chenge the result. Th1s led to the 1dea of using many silts at once, definIng many pairs of beams which would use more of the avallaule light. Thus, as a first experiment, it was ejsential to check 
the effect of changing the position of the beam-defining olit. To do this, a mask with one slit was mounted between the laser and the calcite rhomb. Th1s just produced a two-kean system, but th1s time, in photographing the result, the beam-defining slit was swept ecross the rhomb while the camera was open. Thus, if the pattern hed varied with slit position, the effect woul t have been washed out. Instead, the sharp pattern in FIg. TV-8 resulted. (Again, this is a vertical section of a pettern like those shown In the preceding few figures.) Thls clearly shows thet our essentiel supposition is correct: The position of the slit is inconsequent1al.

Since all of the points behind the rhomb thus give the same observation, one might wonder why the mesk there cannot he removed ent1rely. A pattern produced without the mask is shown in F1E. IV-9. The result shown there is clearly not the same as that of a two-tean system. The reason for this differeuce is that a larger aperture permits the system to focus the light down to a awaller spot. (c.f. FIg. TV-7. What we now have is a pattern which contains less than one fringe.) Thus almost all the sources observed radiate into beam 1 . Th1s in 1teself might be desirable, but the trouble with th1s errangement becomes apparent when the pattern is observed away from focus (Fig. IV-10). There the light is gtill all polarized ae 1. (The order of the two polarizations was reversed between these flgures. The Ilght is, in fact, polarized the same way in Loth cases.) Thus this system does not provide spatial locallzation. Sources all along the 


\section{I}

$r$

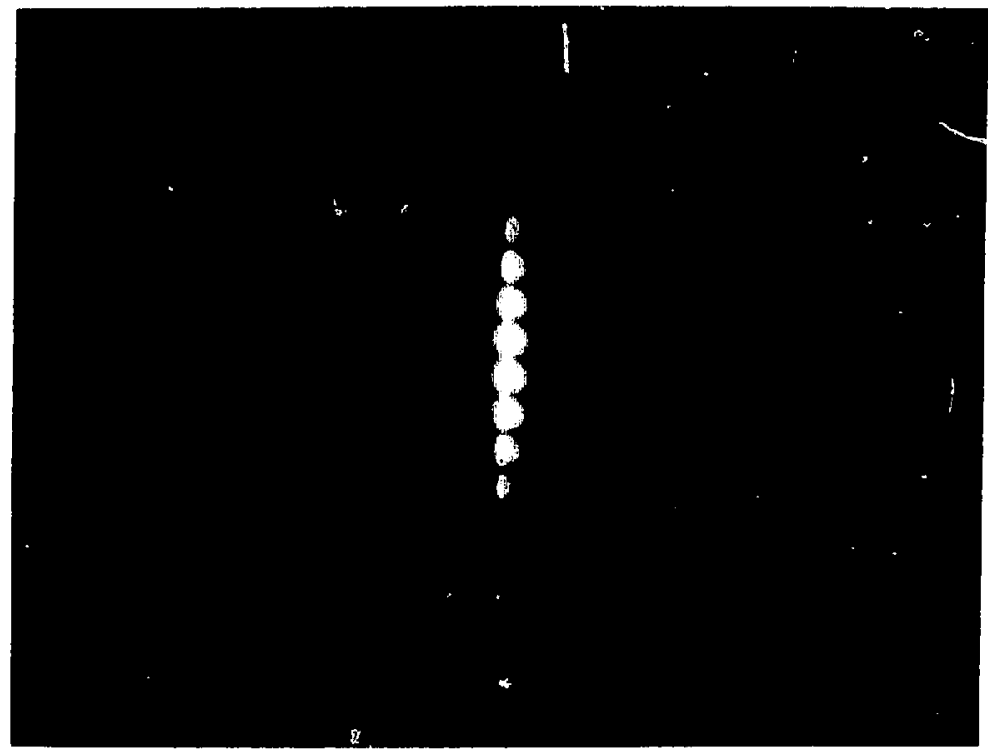

XBB $735-3032$

FIB. TV-8. Lack of dependence of a two-beam pattern upon slit position. A photogreph in which the beam-defining slit was swept across the rhomb during exposure. 


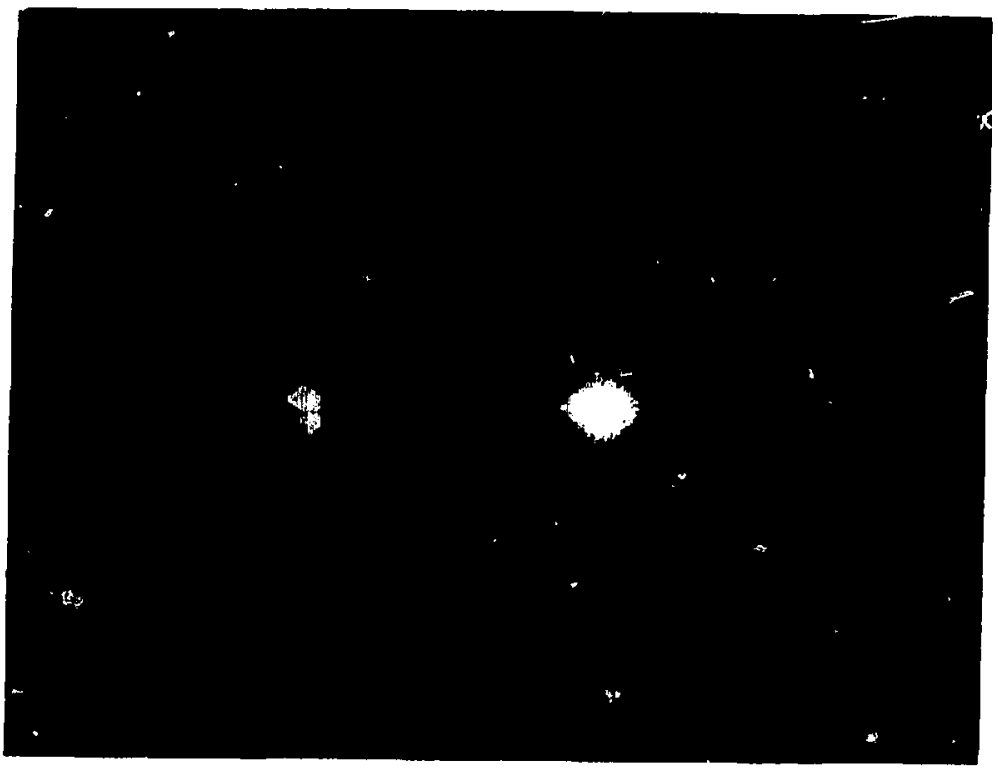

XB $735-3033$

F1g. IV-9. The effect of the whole rhomb without a mask-polarizations 1 and 2 observed at focus. 


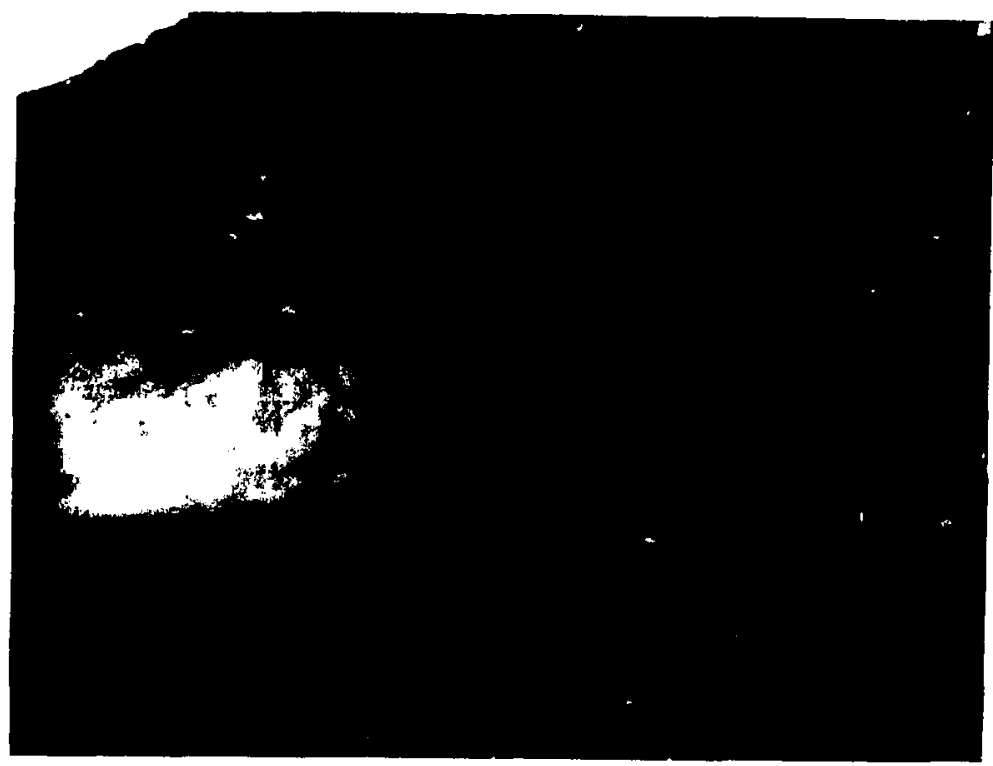

XBB $735-3034$

F1g. IV-10. The effect of the whole rhomb without a mask-polarization: 1 and 2 observed away from focus. 
line of sight would be observed. In the two-bean system, localization vas provided by having separate beams which intersected only near the focus of the system. Without the besk, "beams A and B" completely overlap.

To avold this difficulty one might consider inserting behind the rhomb a mask with many sllts--defining separate beams but using more than two beams to accept more light. The effect of such a system 1s shown in Fig. IV-11.

The result shown there is again different from that of a two-beam system. In the new patterns, polarizations 1 and 2 give narrow, widely separated fringes. The reason for the difference is apparent from the patterns of $A$ and $B$ suown in the lower frame. Unlike the two-beam case, these patterns are not uniform, but now themselves constst of fringes. As explained in Appendix C, these fringes are produced by mult1ple-beam interference of the l1ght transmitted through the different slits. Thus the overall effect Is not the same as would be found for any of the slits considered singly.

This system wes also analyzed in sect. II.B (it is the second of the "three particular systems" of sect. II.B.3) and the conclusion obtuined there is given in Eq. (II.31). In the system of Fig. IV-11, the number of slits, $\mathrm{n}$ was 4 , and $\Delta$, the slit spacing, was approximately twice as much as $d$, the relative displacement of components $A$ and $B$ after the rhomb. When these values are inserted into $\mathrm{EI}$. (II.31), it then describes correctly the difference botween the patterns 1 and 2 in FIg. IV-11. (Note, for 

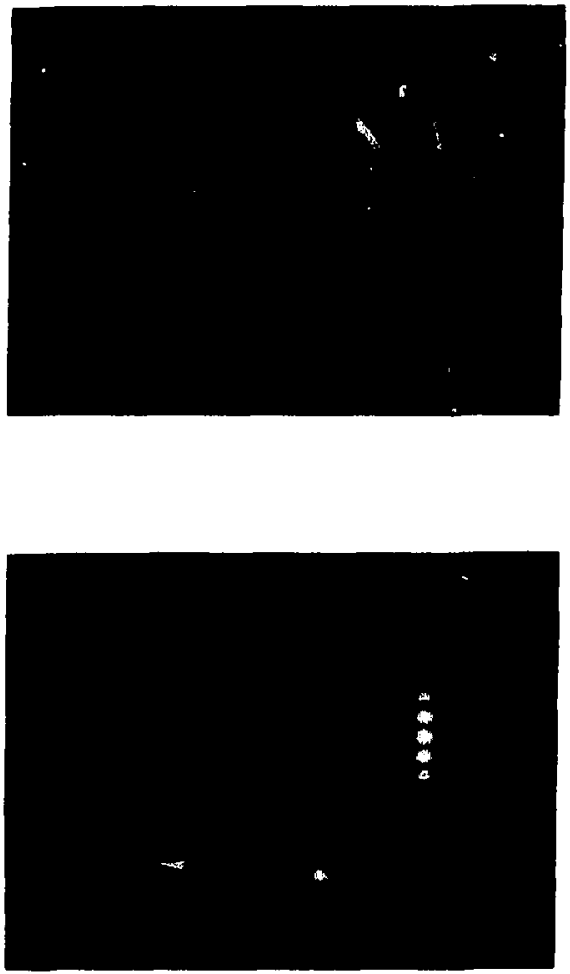

XBB $735-3037$

F1g. IV-1I. Fatterns at the focus of a mult1ple-beam system. Top: polar1zations 1 and 2; bottam: polarizations $A$ and $B$ and total light. Here $n=4$ and $\Delta=2 d$. 
example, thar Eq.(II.31) says that the polarization fringes should be twice as widely opaced ros are the maxtma of total light intensity. In the photographed patterns, this is the case.) Thus this result also agrees with the predictions of our theory.

Although it differs from a two-bean system, this multiplebeam system also defines separate beams and hence should also glve a local meesurement. This it would do, as can to seen from Fig. TV-12, which shows the effect of the same system awky from focus. There beams $A$ and $B$ are phystcally separated, cornponents $I$ and 2 are 1dentically distributed, and hence the difference signal $Y(t)$ would always vanish for sources this far from the focus.

Thus this system could be used to make a local spectroscopic measurement. The result might well be useful, but since it would be due to the irregular component of source density described by Eq. (II.31), the Informatton obtained from this system would be in an inconventent form.

4. A System with Several Independently Collimated Pairs of Beams

In our theoretical analysis it was concluded that one could make a multiple-beam spectroscoplc system which would observe just one $\underline{k}$ component of the distribution of light sources within a local region. This system, which is described in Appendix $C$ and was analyzed in Sect. II.B.3, was to include a collimator, a device which would define completely independently a who? set of pairs of beams A and B. By avoiding any interference between light accepted through the different slits, it was concluded that one could produce a more efficient version of a simple two-beam system. 

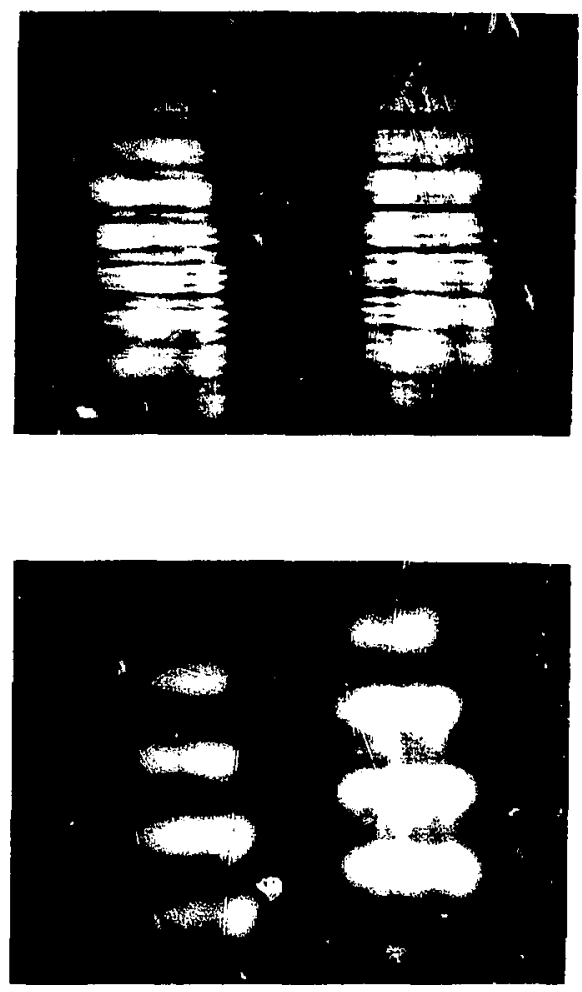

XBB $735-3036$

Fig. IV-12. Patterns at a distance from the focus of a multiple.. beam system. Top: polarlzations 1 and 2; bottom: folarizations $A$ and $B$. Here $n=4$ and $\Delta=2 d$. 
To actually bulld such a system it was oniy necessary to contruct the collimator. Since this device was made with some care, a few vords on 1ts design may be appropriate. The collimator wes made from sheets of $10 \mathrm{mil}$ hard copper. From this were cut thirty plates 1-7/8 $\times 4 \mathrm{in}$. and thirty pairs of specers $5 / 8 \times 4$ in. To form the collimator, these pieces were assembled in a stack of $0.600 \times 1-7 / 8 \times 41 \mathrm{n}$. To make the structure rigid, two pieces of 3/16 in. brass were cut to the length and width of the plates and mounted at the top and bottom of the collimator. To reduce reflections, two holes $5 / 8 \times 1-1 / 16$ in. were cut in each of the 10-m1l plates. (1.e., the center $5 / 8$ in. of the length of each plate consisted of three strips $5 / 8 \mathrm{inl}$, wide, $1-1 / 16 \mathrm{in}$. apart. To cut the holes, the plates were clamped together in a stack and milled.) The assembled collimator was held together with both pins and screws. After assembly the device was electrolytically blackezed in a chemical bath. The collimator ready for use is show in Fig. IV-13.

As a first test, the collimator was used in place of the mask in a multiple-beam system like those discussed in the preceding section. The result is shown in the upper fram of Fig. IV-14. There polarizations 1 and 2 again show sharp, widely spaced fringes. Th1s is not the effect of a system with meny Independently collimated patrs of beams. Since the laser beam was already collimated, the collimator simply acted as a mask with meny slits, and since the laser light was coherent across the wldth of the beam, the light whlch went through different 

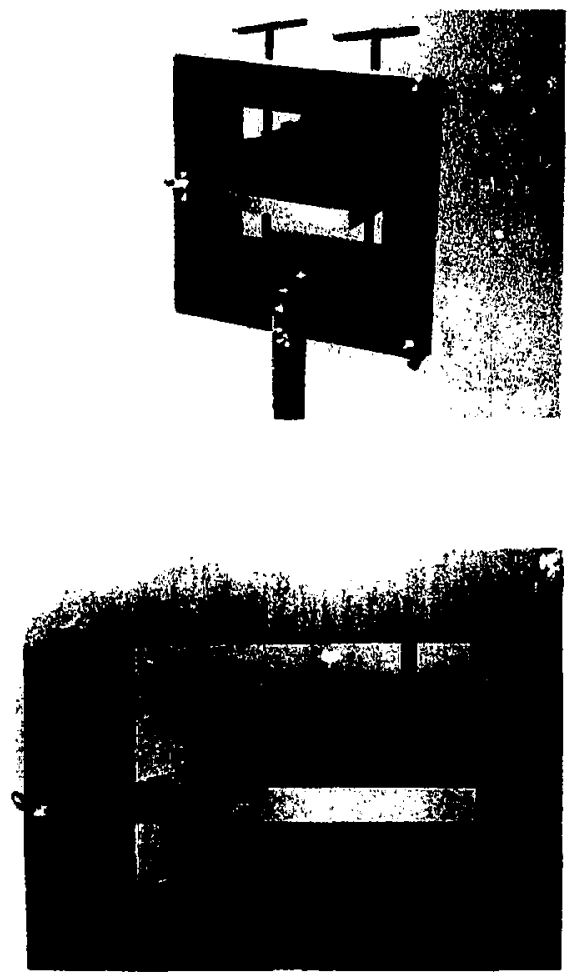

XBB 735-3038

F1g. IV-13. Two views of the collimator. 

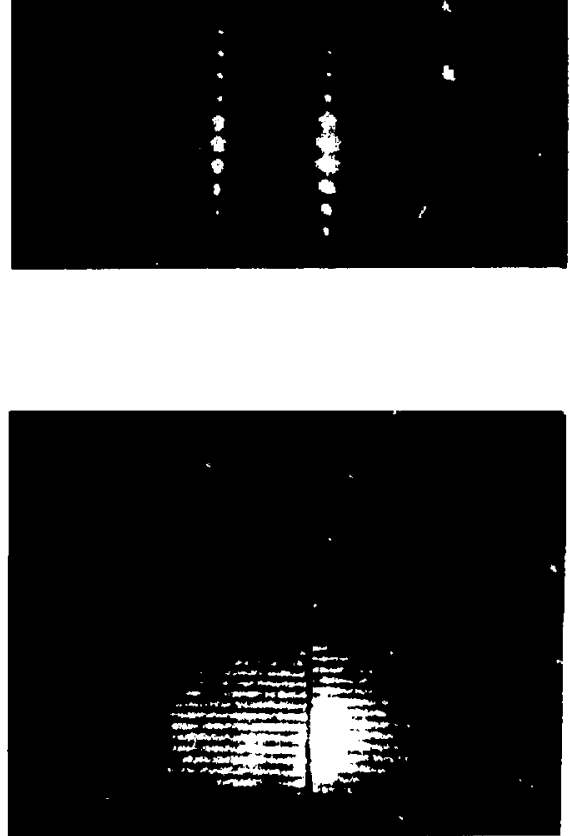

XB B $735-3035$

F1g. IV-14. Two effects of the collimator.

Top frame--a inultiplc-beam pattern, $n=15, \Delta \approx \mathrm{d} / 2$, mede with the slits coherently lllumlnated.

Bottom frame-the effect of many independently collimated patrs of beams.

(Polarizations 1 and 2 shown in each case.) 
collimator slits interfered to give the result shown. This is another example of a pattern described by Eq. (II.3I). Here $\Delta \approx d / 2$ and $n=15$. (The collimator was taller than the rhomb, so only 15 slits were used.) Since, according to $\mathrm{Eq}$. (II.31), the polarization fringe spacing should be roughly half the intensity fringe spacing, one might expect that only one polarization would be seen. This is almost true. Near the center of the pattern the light is mostly polerized as 2 , but since d was not exactly twice $\Delta$, the two patterns "get out of phase" and near the top the light is mostly polerized as 1.

This result again confirws the calculations which led to Eq. (II.31). It also shows what could happen if interference between light eccepted through the different slits of the collimator were permitted to affect the result. In use in a spectrometer, the collimator is to be followed ky a lens focused on the monochromator entrance sl1t. It is absolutely essential that this entrance silt te large enough to accept all of the light transmitted by the collimator. If only part of the Iight at that surface were accepted by the monochromator, the result of the measurement would depend upon multiple-team interference and the observed source density component would be ofmilar to that shown by the difference between the two patterns in the upper frame of Fig. $\mathrm{TV}-14$.

When the collimetor 18 correctly ueed, 811 of the light trensmitted through the different glits will be accepted, so the result w11 not be affected by any wult1pe-lean interference. To model 
this in an inverted gystem, it is necessary to flluminate the collimator incoberently. This was done by shining the laser onto a ground glass screen. A lens, focused on the screen, then gave an approximately collimated beaw, but one in which the light was spread over a range of directions greater than that accepted by the collimator. Altogether, the test systen was:

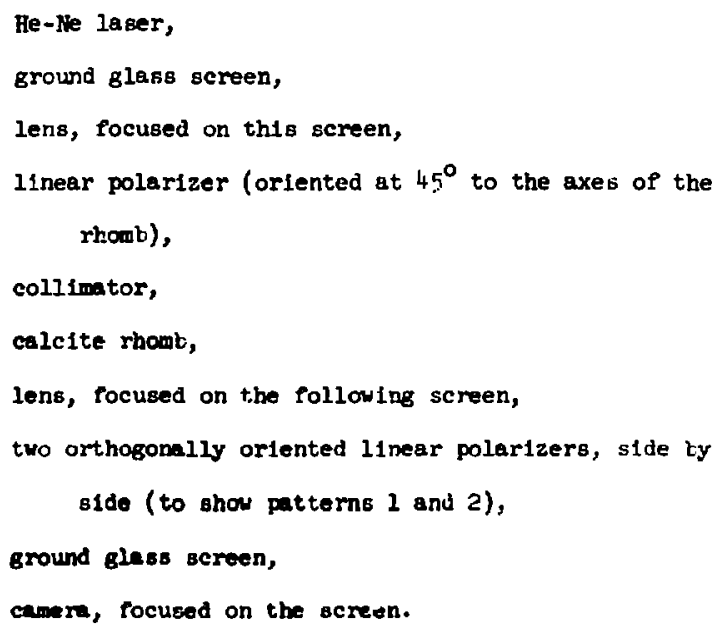

The result of this aystem is sbown in the lower frame of F18. IV-14. There polerizations 1 and 2 show simple sets ar fringes like tbose otrained in the two-bear oystew. Thls is the desired result. In use as a sectrometer, this system vould select - alnele 2 componant (bore preciaely, a narrow range of components) of the distribution of light scurces within the region observed.

This oynters in discuisod In Appendix ce and lu fect. TT. R. 3 , where the conclualon In givon ly Eq. (II.33). The patterni: In 
F1g. IV-14 egree with the predictions of the theory. (The Pinescale graininess is just lasar apeckle, which has nothing to do with the spectroscoplc system. Use of a ground glsss gnreen does not really make the laser light incoherent, it merely complicates the phase relations so that unwanted interference produces only this easily ignored effect.)

As with the other systems tested, this result confirms the theory and also shows that the optical components were of sufficient quality for use in such a system. In the present case, this was particularly important, because in the formal theory it was assumed that all peths through a given collimator slit were equal to within a fraction of a wavelength, rut the collimator actually used was not quite this restrictive. This difference did not appear to affect the result. As can be seen from Fig. IV-14, the fringe patterns produced agreed quite well with the predictions of the theory.

\section{B. Observations of a Plasma}

In the rinal part of the experimental work, the last optical system tested was used to observe fluctuations in a plaswa. The plewe which wes ueed for this purpose was produced in the Berkeley electron beam-plsome mochine, which had previously been ueed in other experleontal work. ${ }^{51}$ Photogrephe of the mechine and of the plasme are eboun in Fig. IV-15.

In this device, an electron gun, biased to $4 \mathrm{kV}$ negative, 

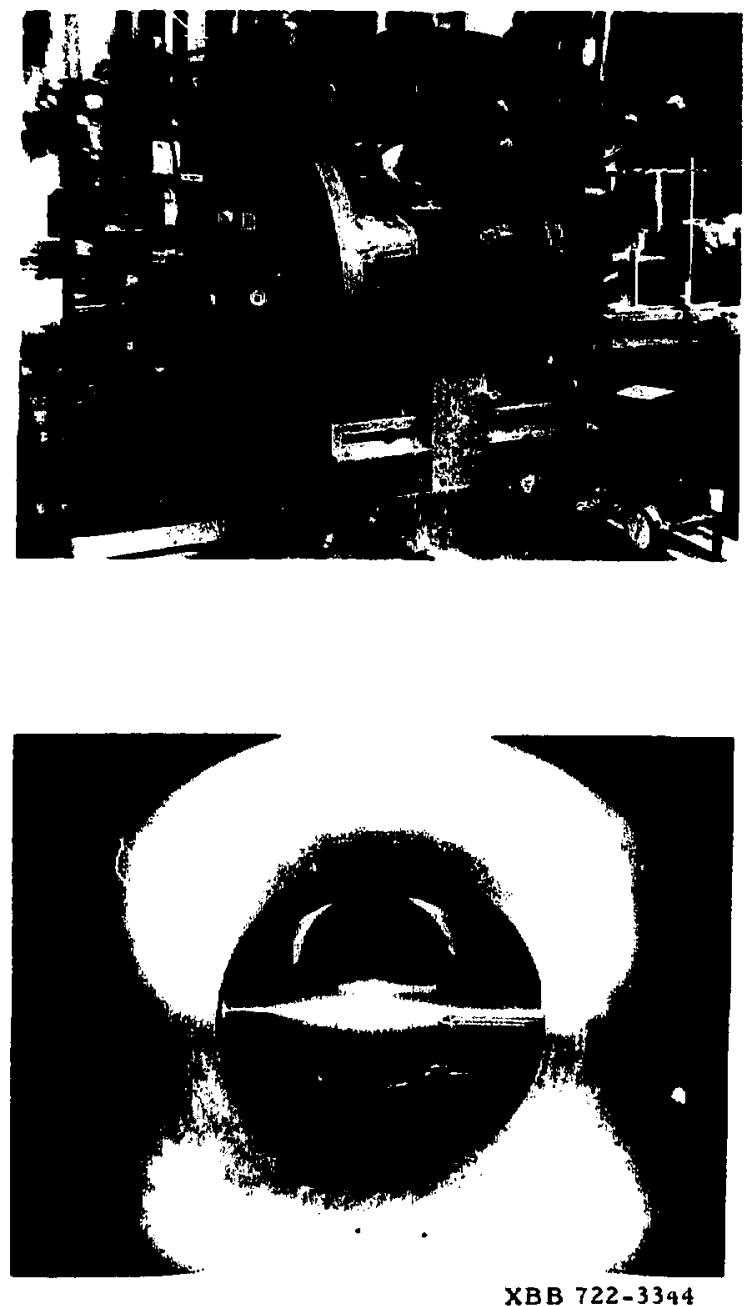

Fig. IV-15. For legend, see page $134 a$. 
F1g. IV-15. The electron beam-plasma expe:1ment. Top: The machine. The electron gun is to the left of ihe glass tee. The flasma chamber is between the two Large magnet colls.

Bottom: Plasme with probes. 
produced a $30 \mathrm{~mA}$ bean which w s injected through two successive apertures (separating three Independently pumped vacuun chambers) Into a chamber filled with 300 mlcrons of hellum. The resulting beam-plesma instability produced a plasma with an electron temperature of a few eV and an electron density of a few times $10^{13}$ $\mathrm{cm}^{-3}$. (See Ref. 51 and Appendix D below.) The plasma was confined by a ragnet1c fleld of $7 \mathrm{~kg}$ produced by two colls in Helsholz conflguration. (The magnetic fleld also served to gulde and focus the electron beam.) The electron beam was less than 1 min in dianeter and the resulting cylindrical plasma was approxinately $0.5 \mathrm{~cm}$ in diameter and more than $10 \mathrm{~cm}$ long.

For a controlled test of the spectroscople system, it was desired to produce in the plesma a disturbence of known frequency and of relatively large amplitude. Thls was done by using a negatively biased Langmulr probe with which one could vary the plasma density. This technique had already reen used successfully to study the propagation of pulses in this plasina (see Appendix D). The pulse propagation work had shown that a density perturtation would be transmitted through the plasma at a speed slightly in excess of $10^{6} \mathrm{~cm} / \mathrm{sec}$, the expected ton sound speed.

In the multiple-beam spectroscopic observations a sinusoldal oignal rather than a pulsed signal was used. Frequencles tn the range 10-50 MHz were chosen, since at the indicated 1on sound speed this would glve disturkances with wevelengths of a fraction of a millimeter--a convenient wavelength to observe with the mult1ple-beam system. 
To improve the signal-to-noise ratio, two stages of phasesensitive detection were employed. First, the transmitted signal was modulated at $50 \mathrm{kHz}$ and th1s modulation was used as the reference aignal to a luck-In emplifier. Secondly, the observed I1ght was modulated at $1 \mathrm{kHz}$ with a mechenical cinopper and a reference stgnal from the chopper was used by a second lock-in amplifler (both were PAR Hr-8's).

The spectroscopic system was tuned to the neutral helium line at $4471 \%$. To provide the needed aperture, the entrance and exit slits of the monochromator were removed entirely, a change wh1ch did not apprestably reduce the coherence length of the light, since most of the light was still in the $4471 \&$ line (see Appendix D). The neutral density was not expected to vary with plasma denstty, but since the neutral light emlission was caused by excitation of neutral atoms by the plasma, it was expected that the light intensity would vary with the fluctuations in the plasme density.

The plasma was observed from a distance of $61 \mathrm{~cm}$ through a $13 \mathrm{~cm}$ focal length lens. This was followed by another lens of focal length of $26 \mathrm{~cm}$ which Imaged the plasma at infinity. Later in the eystem, this light passed through the calcite rhomb of $1 \mathrm{~cm}^{2}$ aperture which dioplaced one polarlzation laterally by 1.1 m. In the multiple-beam systom the effect of this was to select frow within the plasme a source-densily couponent of wavelength of $0.391 \mathrm{~mm}$ and to observe this through an uperture $0.64 \mathrm{~cm}^{2}$, $61 \mathrm{~cm}$ from the pleame. 
The collimator, the rhomb, and the monochromator were mounted Bo that beams $A$ and $B$ would be horizontal fans, vertically displaced. This would permit one to observe a vertical $\underline{k}$ vector component of the source distribution. Since the magnetic fleld lines in the plasma were horizontal, and since it was desired to observe propagation along the fleld, a Dove prism was included in the system to rotate by $90^{\circ}$ the Imege of the plasma.

Altogether, the opticel train was:

plasma,

lucite vacuum window, objective lenses

linear polarizer (oriented at $45^{\circ}$ to the axes of the rhomb),

celcite rhomb,

collimator,

lens, focused on the entrance to the monochromstor, mechanical chopper,

monochromator,

Glan-Thompson prism (separating polerizetion components 1 and 2),

lenses, focused on the ex:t of the monomchromator, photowultiplier tubes.

The last fow elewents in the optical train are shown in Fig. IV-16. There can be seen the monochrorator, the preceding lens, and chopper whel, and the "Y" structure containing the GlapThompson prlan, the two lenses, and the two photomultiplier tures. 


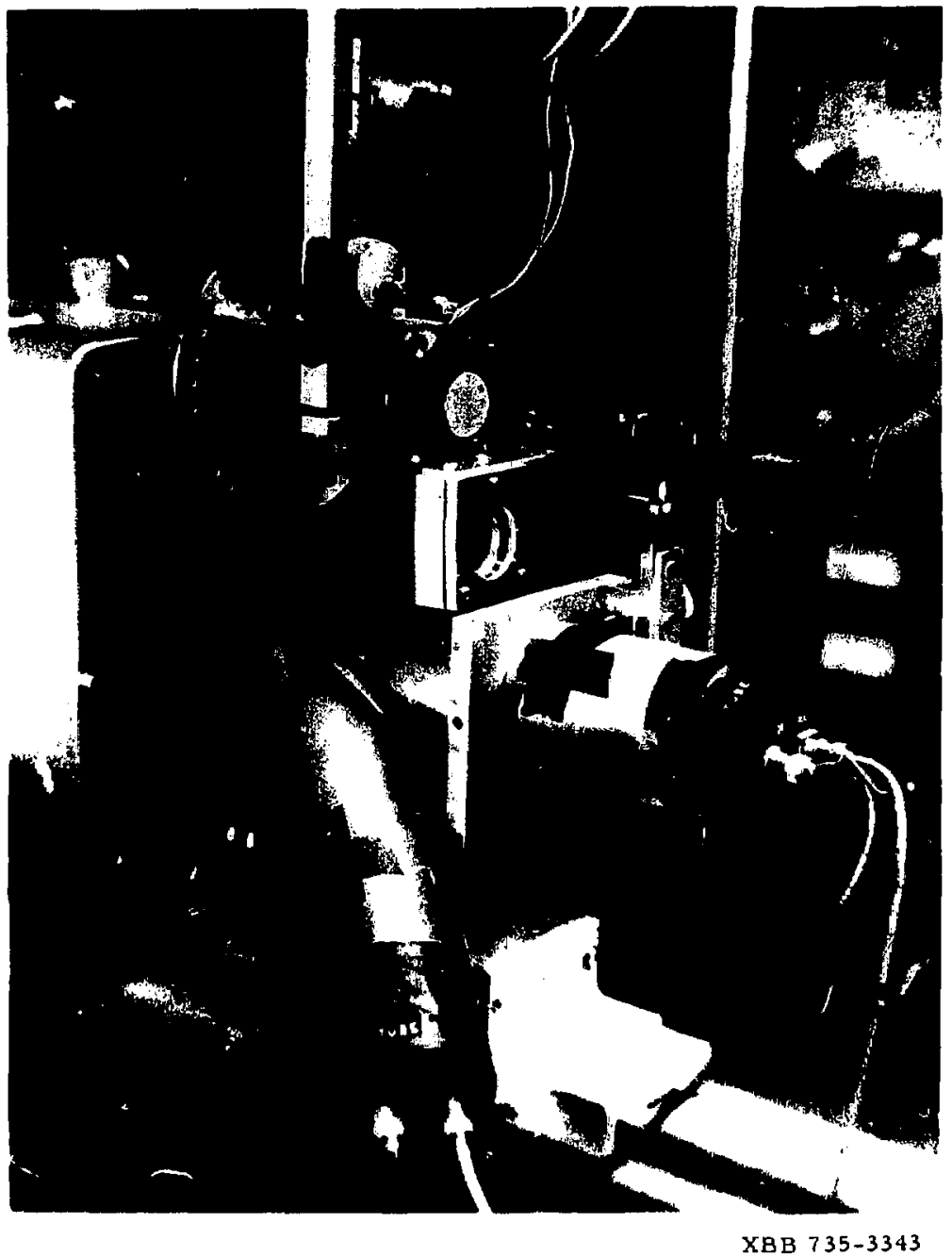

Fig. IV-16. For legend, see page 138a. 
Fig. TV-16. A view of the opticel system showing the chopper wheel, the photomultipl ler tuke assembly, and one end of the monochromator. 
A diagram of the entire epperatus is given by Fig. IV-17. As Indicated there, the phototube outputs were compared by a differential amplifier, the resulting signal was fed into a spectrum anelyzer, the output of this was processed by two lock-In ampliflers, and the result of this was then plotted by an $X-Y$ recorder driven by the spectrum analyzer sweep.

The output was thus in the form of signal spectra. The disturbance in the plasma was injected at one frequency and the opt 1cal system was designed to observe one $\underline{k}$ component of the resulting plasme oscillations. Hence if the transmitter produced a disturbance at the observed $k$, the stgnal spectrum would show a peak at the infected frequency.

Such a result is seen in Fig. IV-I8. Each of the signal spectra shown there covers a range $39.5-41 \mathrm{MHz}$, which includes tie transmitter frequency ( $40 \mathrm{MHz}$ ). The phototule difference signal (1.e., the intended output of the system) is shown in the upper trace, which does in fact exhilit a peak at the imposed frequency. This peak disappeared when the light peth was blocked and it disappeered when the transmitting probe was rotated out of the plasma. A spectrum of the output of a single one of the phototuies 16 shown by the seccnd trace in Fig. IV-18. There the amplitude of the peak is reduced $k y$ half. This is as expected, since a wave at the observed $\underline{k}$ in the plasma will cause the light to oscillate between the phototubes (1.e., between the "interference patterns" observed by the phototubes). In the intensity difference signal, these two oscillations: which are out of phase, will add. So in 


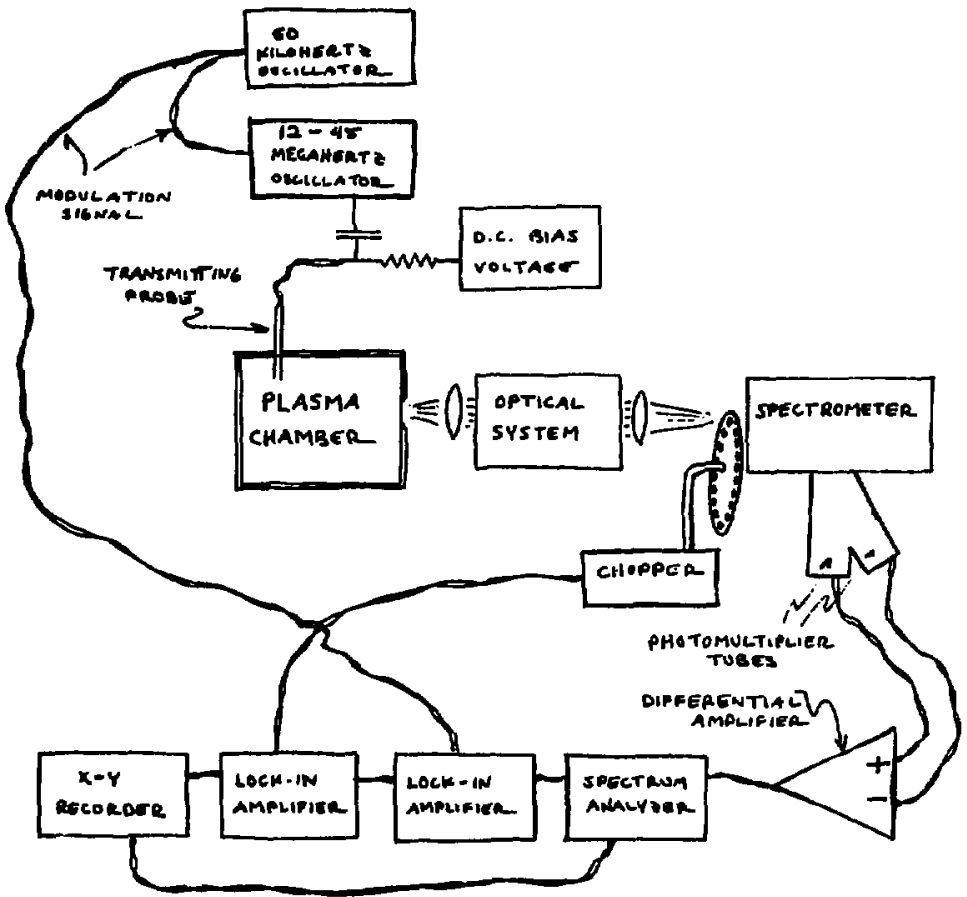

XRL 735-669

F1B. IV-17. Prinelpal elements of the apparatus. 

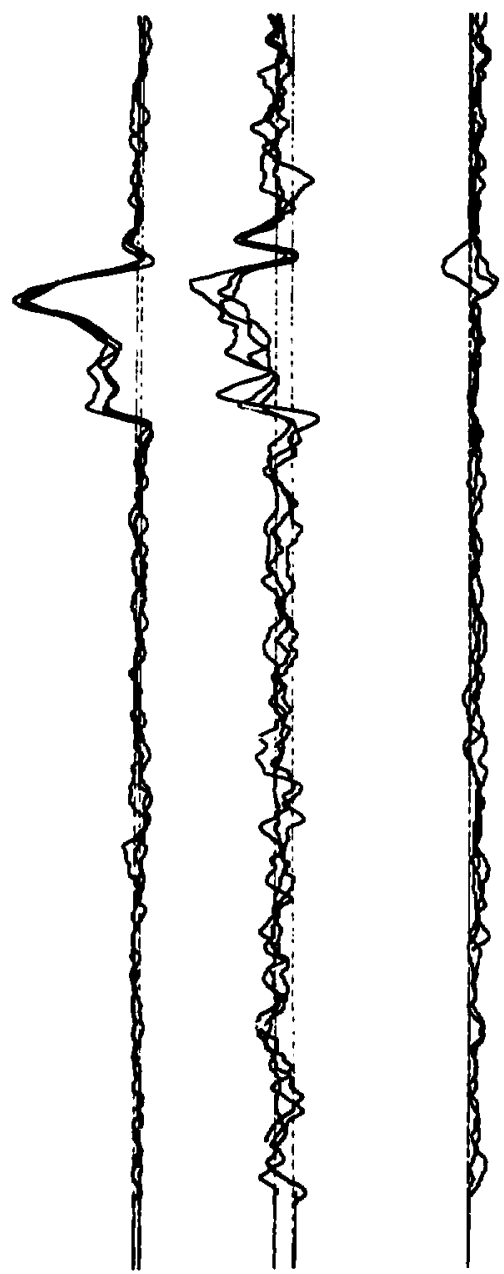

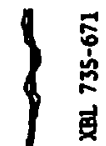

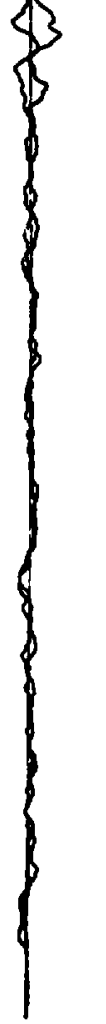

FIg. IV-18. For legend, see page 1410. 
Hig. IV-18. Blgnal spectre (approxlmately $3 y .5 \mathrm{MHz}$ w1th lower frequencies at right).

Top trece: The intensity difference signal.

Second trace: The output of one photomultiplier tube.

Thind trace: The intensity difference signul with the

light path blocked.

Bottow trace: The output of one photomultiplier tube with the 11ght path blocked.

Each trace is three spectra superimposed (except the bottom trace, wh1ch is tho spectra). Th1s is a photograph of the $X-Y$ recorder graph. 
the spectmun of tine output of each phototube alone, one ought to see exactly half the total signal amplitude.

of equal intereat is the change in the nolse level all across the spectrum. The second trace is clearly notsier then the first. Nothing in the apparatus uas charged between these to measurements (actually six measurements, as each spectrum is three traces). The differential amplifier was simply switched from (2-1) to 2 . The increased noise if due to random variations in the plasma luminosity. When the two phototube signals are afferenced, the Pluctuations in the total light level are cancelled out. When only one of the tubes is used, the nolse in the output is much greater. This is a useful thing to note in setting up the apparatus, because the cancellation of the nolse shows that the system 1s correctly balanced at the frequencles of interest.

To be sure that the increased nolse was not an electronic effect, the same spectra were again recorded, with the light path blocked (transwitter still on). The result is shown in the third and bottom sets of trances in Fig. IV-18. There the noise in the output of one phototube is less than that in the intensity difference signal, just as one would expe't. Interestingly enough, the noise in the third set of traces (th. difference signal with the light path blocked) Is not much less than that in the top set of traces. Evidently most of the noise in the system came from the electronics, and hence it could heve been eliminated by refinements in the epperatus.

On close inspection of the first two sets of traces, one sees 
two small peaks, one on either side of the main peak, which increased when the system was owitched from (2-1) to 2 . Unlike the random nolse, these features show a oygtematic change. This almost certainly io due to modulation of the total plasma luminosity by the transmitter. In Langmulr probe observations of pulse propagation In th1s plasma (see Appendix D) a fast signal was always seen. This was attributed to a potential fluctuation. That alone should not change the light emission, but there certainly are other mechanisms, such as a change in the electron temperature, which would cause the fast signal to make at least a slight change in the plasma luminosity. Since this vould be seen with the same phase by both phototubes, the efiect would be seen by each one alone, but would be belanced out in the intensity difference signal.

The presence of what appear to be the same two small peaks in the first set of traces may be due to a slight imbalance between the phototubes. A second possibility is that the plasma oscillation at the observed $\underline{k}$ merely had simlar components $\mathrm{n}$ its spectrum 1.e., that the second set of traces is the sum of two components, one equal to half the upper traces and the other caused by modulation of the total luminosity.

There 1s, however, a third possibility which should also be mentioned. If there vere a stationary or a slowly varying plasma diaturbance at the observed $\underline{k}$, then the total light from the plasma would be divided unequally between the two phototubes. This by Itself is juat like any other flustuation which the spectroscopic 
system might observe. In this case the effect would appear at the low-frequency end of the signsl spectrum. However, if the total plasma luminosity is modulated at a higher frequency, then the phototube whlch sees more light wili see the modulation with a larger amplitude, and hence the modulation will appear in the intensity difference signal.

In other words, a h1gh-frequency modulation of the total plasma luminosity would "Illuminate" low-frequency density varietIons and these would then appear to have the higher frequency. Now, as far as the optical analyo1s is concerned, there is nothing to explain here. The high-frequency intensity modulation and the low-Irequency density inhonogenelty comblne to produce a fluctuation of the light source density at the observed wavelength and frequency. Then $n_{s}(\underline{k}, \omega)$ actually exists in the plasma, and so, of course, the multiple-beam system sees 1t. But in 1nterpreting such results it is 1mportant to realize that not every observed fluctuation corresponds to a wave in the plesma (except, perhaps, In a very broad sense of the term). Some features in the signal spectrim could be due to fast alsturbances illumfnating slower ones.

In our data, the location of the two small peaks at the sides of the main peak is not necessarily an indication of a difference In frequency. These data vere taken with a lock-1n awplifler which selected one phase component of the output of the spectmm analyzer. since the phase and the amplitude of the $(50 \mathrm{kHz})$ analyzer output woulc both change as the analyzer swept in frequency, the exact 
shapes of features in these spectre did vary somewhat with the phese setting of the first lock-1n amplifier. All the curves shown here were taken at a single phase setting.

It is also of Interest to note the amplitude of these osc1llations. When the gains of all the elements in the system are considered, the amplitude of the largest signals observed (at $29.5 \mathrm{MHz}$ ) is found to correspond to about, a $10^{-10} \mathrm{~A}$ oselllation in photocurrent. The mean phototube output (measured directly) was $10^{-7}$ A. Hence the atrongest signals were due to a $0.1 \%$ oscillation of the observed light intensities. In the pulse propagation studies (see Append1x D) in which the transmitter voltage was roughly the same as that used here, the peak of the pulse Ident1fled as an fon wave also represented a $0.1 \%$ change in the probe current. Now the width of that pulse was about five times the width of the transmitted pulse, so this amplitude represented about one-fifth of the whole disturbance. Judging by the interference patterns photographed in the test program (see Fig. TV-14) the epectroscoplc system nould observe a region about 40 fluctuation wavelengths wide. Thus the resolution in $k$ was a few percent. Bence if the spread in transmitted wavelengths were about $10 \%$, the spectral amplitude would also represent one-fifth of the disturbence. In a sweep through different frequencles (see F1g. N-19) the maximum seen around $29.5 \mathrm{MHz}$ did extend over roughly a $10 \%$ range of erequencies.

So the amplitude of the pulses seen with probes was roughly equal to the amplitude of oscillations observed spectroscopically. 
In fact, th1s agreament must be at least in part fortultous, if only becsuse the dependence of the light intensity upon the p'asme density is not known. An sttempt was made to measure this dependence by changing the transmitter amplitude. It turned out that at large amplitudes (larger than where the date shown hare were taken) the spectroscopic olgnal actually decreased as the transmitter amplitude increased. At lower amplitudes, the signal increased with the oscillation voltage, but the increase was much more rapid than linear. So the amplitude callbration has not been related to the plasma density disturbance. Still, it is worth noting that the observed $0.1 \%$ modulation of the light is reasonable and is similar to the density modulations seen with probes in the pulse studies.

Finally, to gain some information about the plasma response, the amplitude of these signals was observed over a range of frequencies. For each measurement the transmitter was set at one frequency and the spectrum analyzer was swept past that frequency. A set of the results is shown In Fig. IV-19. There one can see that the amplitude of the observed response did vary quite sharply with erequency. of particular interest is a peak in response at bout $29.5 \mathrm{MHz}$ (the seventh trace in this set). Since the observed vavelength was 0.391 mat this froquency corresponds to a phase velocity of $1.15 \times 10^{6} \mathrm{~cm} / \mathrm{sec}$. This is roughly equal to the ton sound spoed, as computed from the measured temparature and as obeorved in the pulse propegation studies (see Appendix D). This susgests that these date show plasm density disturbances trans- 


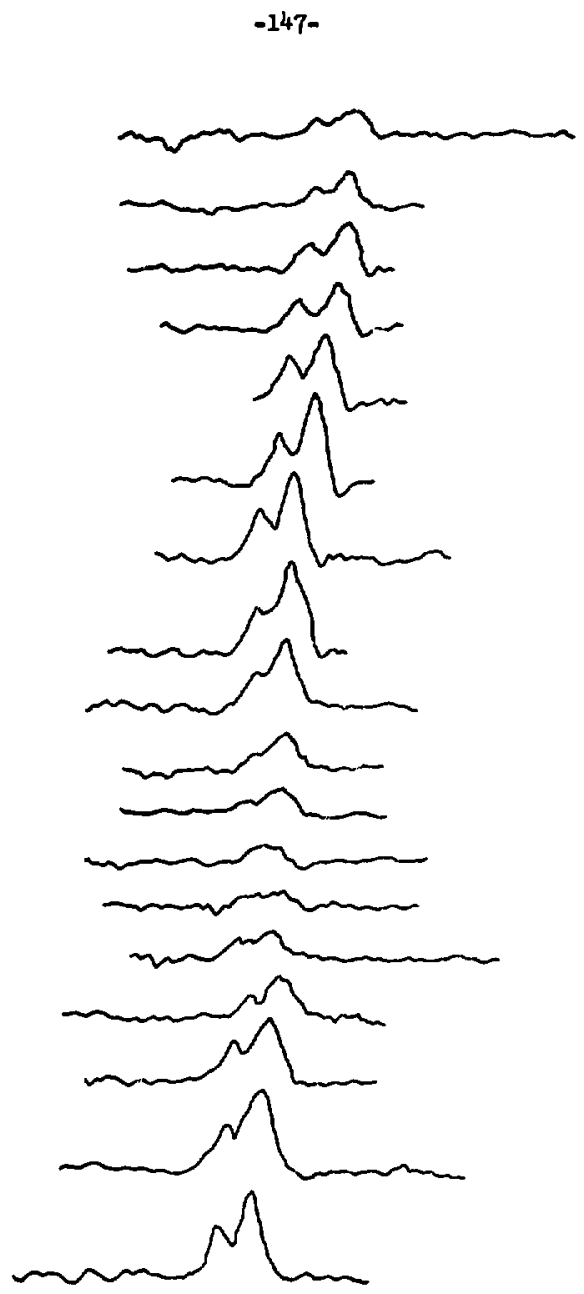

XB1 $735-672$

F18. IV-19. For legend, вee page $147 \mathrm{a}$. 
21g. IV-19. Signal spectra taken at $0.5 \mathrm{MHz}$ intervals from 26.5 through $35 \mathrm{MHz}$. These are tracings of the $X-Y$ recorder plots. 
mitted at the 1on sound speed.

There are, however, several unknowns in the problem. The electron temperature, and hence the sound spped were known to vary witk the distance from the center of the plasme column. The probe pulse data were taken gust outs1de of the electron beam. The spectroscopic meesuremeits presumbly refer to the same region, if only because the transmitter was located there, but the effect of the hotter plasma within the beam remaine unknown. Furthermore, the efficiency of the probe as a transmitter may be frequency dependent. Some of the observed variation in signal amplitude could have been due to the transmitter, rather than to prcrgation properties.

Clearly, an understanding of the dymamics of this plasma would require much more information that is contained in the se few spectra. One could proceed now to use this spectroscopic system to do a complete study of the plasma--looking at different wavelengths, different frequencies, and different porticns of the plasma colum. But this would be a project in itself. Our purpose here is to show the utility of the spectrometer. These data should serve to illustrate the kind of information which can be obtained with such a diagnostic instrument. 


\section{HIGH-FREQUENCY PHENOMENA}

\section{A. The Etfect of a Moving Source ard the Use of a}

\section{Time-Verying optical system}

The need to observe relatively Lifh-frequency phenomena is a undamental consideration of plasma diagnostics. Characteristic frequencies of meny laboratory plastuas lie in the megahert,z or g1gahertz range. In pulsed experiments, the ent1re plasma may exiat for only a small fraction of a second. We have noted alreedy that the need for $p$ probe with a rapid response suggests, In general, the consideration of optical diagnostic techniques.

In the multiple-beam spectrometers described in Chapter II, a component of the light would oscillate between two photomultiplier tubes in a manner characterlatic of one component of the source distribution. Frequencles of plasma uscillations would be observed in the time dependence of the outputs of the phototubes. Yet phototubes, and other elements of the system, ha. $\geq a$ finite bondwith which would, in practice, interfere with the moasurement of rrequencies above a few bundred megahertz. Th1s 1. a serious limitation.

The present discussion began with an amalysis of a simple two-beam spectroweter. A two-beam spectroscopic measurement was found to resemble a light-scattering experiment in several ways. A scattering mesurewent, however, is not restricted to low-frequncy phenowose. Indeed, scattering provides a most convenfent wy to measure higher frequencies, which can be seen as opt Icel frequency difforences in the Doppler brosdened spectrum of the 
scattered light (see sect. I.C). On the other hand, there are scattering measurement technlques (which we have not discussed) In which one ioes observe directly in a measured light intensity the time dependence of low-frequency phenomena. (see, for example, Ref. 52.) This similarity suggests that the low-frequency restriction of the multibeam spectrometers of Chapter II is due to our cholce of apparatus, and not a necesary feature of a spectroscopic measurement.

To see how higher frequency, or higher phase velocity phenowena wight be observed spectroscoplcally, consider first the effect of a single moving source. In Fig. V-I Is shown a small monochromatic light source which woves with a velocity $\underline{s}_{s^{*}}$ If the source has frequency $\omega_{s}$, then the light emitted in a direction $\hat{k}_{A}$ must heve a Doppler shifted frequency (to f1rst order in $\left.\left|\underline{v}_{s}\right| / c\right)$

$$
\omega_{A}=\frac{w_{B}}{1-\frac{\underline{v}_{B}}{c} \cdot \hat{k}_{A}} .
$$

Light which 18 emitted into different directions $\hat{k}_{A}$ and $\hat{k}_{B}$ will differ in erequency by an amount

$$
\begin{aligned}
\Delta_{w}=\omega_{B}-\omega_{A} & =\omega_{B}\left(\frac{1}{1-\frac{v_{B}}{c} \cdot \hat{k}_{B}}-\frac{1}{1-\frac{v_{B}}{c} \cdot \hat{k}_{A}}\right) \\
& \approx \underline{v}_{B} \cdot\left(\underline{k}_{B}-\underline{k}_{A}\right)=\underline{v}_{B} \cdot \underline{k}_{\Delta^{*}}
\end{aligned}
$$




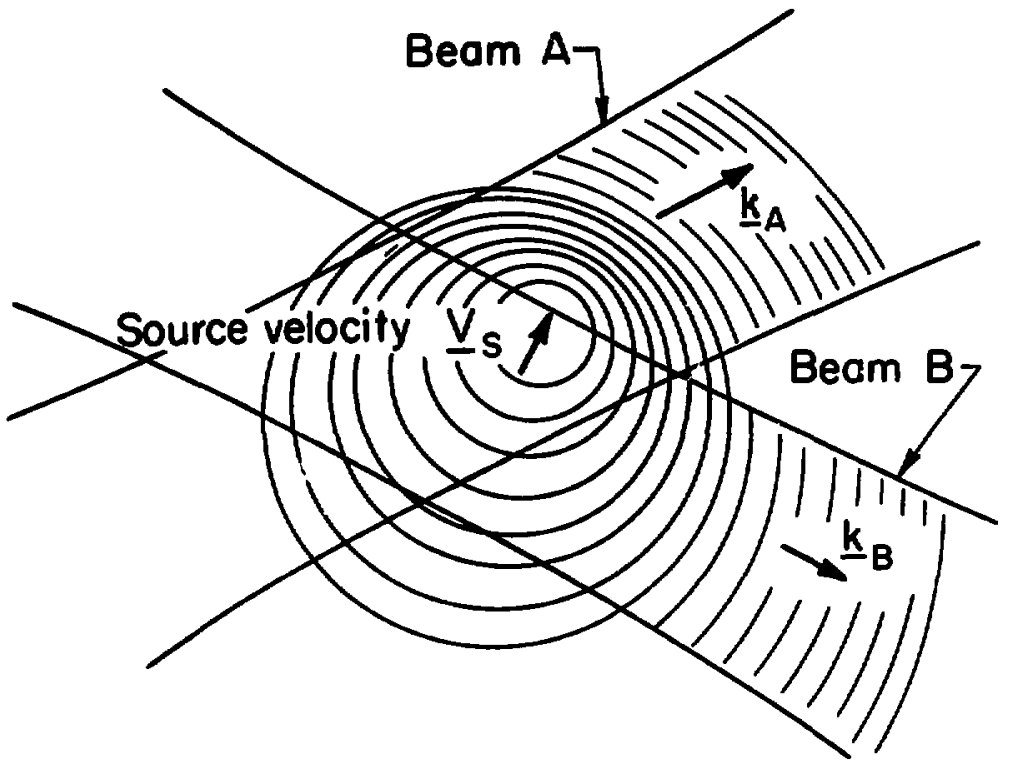

$\times 8 L 733-2515$

Pig. V-1. Two-beam observation of a moving source. 
Bere $\mathrm{k}_{\Delta}$ is the familier difference wave vector c.f. Eq. (I.9) or (II.18)].

So, at least for nonrelativistic motion, the difference in frequency which would be seen in a two-beam observation of a moving source depends upon the same $k_{\Delta}$ whlch describes the fluctuat1ons in source density observed in the low-frequency limit. The same source wevelength characterizes both the mutual coherence and the frequency difference observed with a two-beam spectrometer. This suggests thet it should be posstble to extend the low-frequency technique and observe rapid motions of a light source distribution by comparing light emitted in different directions at different optical frequencles.

In fact, the low-frequency system discussed in Sect. II.A. already involves exactly this. In the simple two-beam arrangement, the observed beams A and B were combined and their superposition was separated Into two complementary interference patterns--beams 1 and 2. It was shown thet an oscillation of the $k_{\Delta}$ component of the light source density would produce a corresponding osc1llation of the Ifigt interisity between beams 1 and 2. This oscillation of the I1ght is due to a steady variation in the relative phese-more precisely, in the phase of the mutual coherence--between beams $A$ and B. Yet a stegdily incressing phese difference is exactly the same thing os a difference in frequency. The lowrequency system simply measures a small frequency difference by observing the time dependence of the beats which result when the two waves are combined. 
One can certeinly messure differences in frequency between the two beams, but to extend the concept of a multiple-beam spectrameter, the phese must also be observed. The optical systems described in Chapter II were designed to measure the routual coberence of light of equal, or at least nearly equal frequency. An obvious way to extend the method is to add to the system a moving mirror or other time-varying element which would Doppler shift the frequency of one of the beams. The remainder of the system could then be left unchanged.

Such a modified two-beam arrangement is shown in Fig. V-2 . The spectral filter again accepts the same $|\underline{k}|$ components of each bear, but since beam B if first shifted in frequency, the light accepted through the two beams is emftted at different frequencles trom the plasma.

The analysis of Sect. II.A.3 is easily amended to describe th1s new arrengement. The light accepted through beam $A$, 18, as before

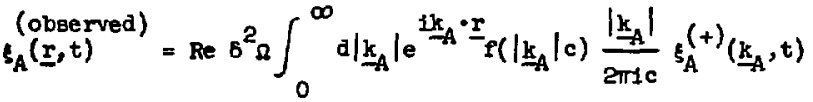

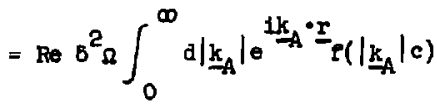

$$
\begin{aligned}
& \text { - } \frac{\left|\underline{k_{A}}\right|}{2 \pi t c} \int d^{3} r^{\prime} \xi_{B}^{(+)}\left(\underline{k}_{A}, t ; \underline{r}^{\prime}\right)
\end{aligned}
$$

Becsuse of the moving mirror, beam B 18, in effect, observed from - moving frame of reference 


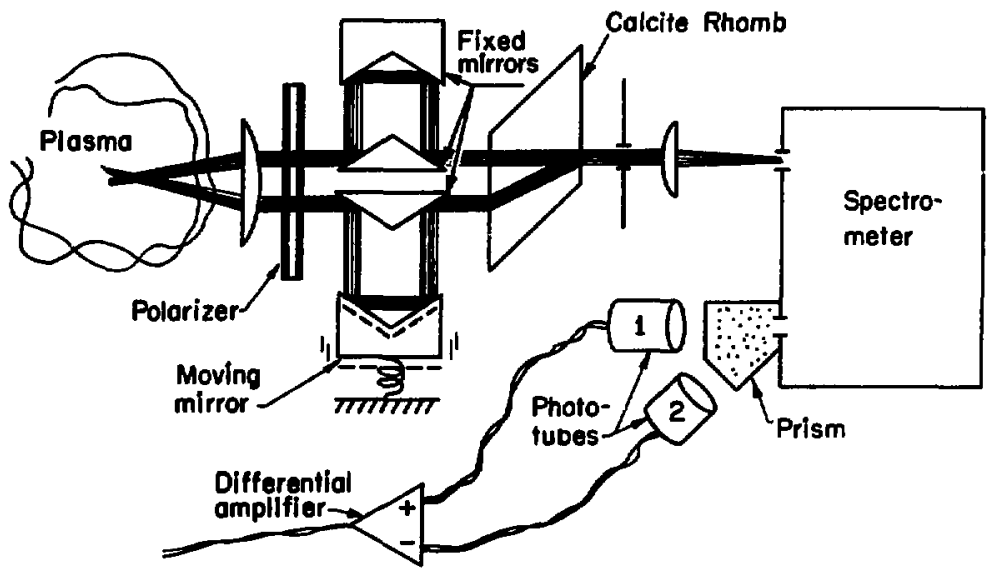

xeL733-2514

FIg. V-2. The use of a moving mirror in a twa-beam system. 


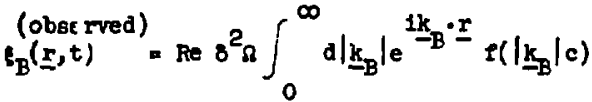

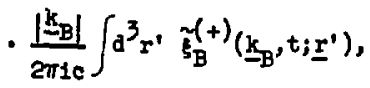

where

$$
\begin{aligned}
& \tilde{s}_{B}^{(+)}\left(\underline{k}_{B}, t ; \underline{\underline{r}}^{\prime}\right)=\int \mathrm{a}^{3} \underline{r}_{1} e^{-\underline{\underline{k}} \underline{k}_{B} \cdot \underline{r}_{1}} \xi_{B}^{(+)}\left(\underline{r}_{1}+\underline{v}_{0} t, t ; \underline{\underline{r}}^{\prime}\right) \\
& =\int a^{3} \rho e^{-1 \underline{k}-\underline{x}_{B} \cdot \rho} e^{+\underline{i k}-\underline{k}_{-} \cdot \underline{v}_{0} t} \xi_{B}^{(+)}\left(\underline{\rho}, t ; \underline{x}^{\prime}\right) \\
& =e^{+i k_{B} \cdot \underline{v}_{0} t} \xi_{B}^{(+)}\left(\underline{k}_{B}, t ; \underline{\underline{r}}^{\prime}\right) \text {. }
\end{aligned}
$$

Bo, the effect of the moving mirror is to introduce in the observed field amplitude an additional factor of $e^{-1 k_{B} \cdot v_{0} t}$.

As before, the two weves are combined to produce two interference patterns

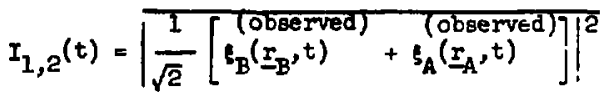

and the difference between these intengitieg provides the output of the system,

$$
Y(t)=I_{2}(t)-I_{1}(t)
$$

Assuming that $f\left(\left.\right|_{\underline{x}_{A}} \mid c\right)=f\left(\left.\right|_{-B} \mid c\right)$, describing the intensity In terms of an analytic signal, and assuming again that the 11ght frow each roint $\underline{I}^{\prime}$ 19 coberent, but that light frow different 
points is incoherent, leads, as before, to the expression

$$
Y(t)=\int_{\substack{\text { comron source } \\ \text { volume }}}^{d^{3} \underline{r}^{\prime}} \int_{0}^{\infty} d|\underline{k}||f(|\underline{k}| c)|^{2} \operatorname{Re} \tilde{\Gamma}_{B A}\left(0 ;|\underline{k}|, \underline{r}^{\prime}\right)
$$

where now

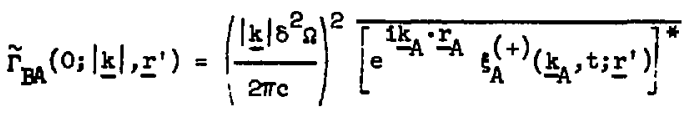

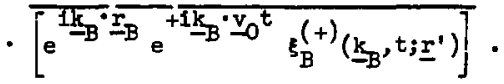

Expressing $\xi_{A, B}^{(+)}$in terms of $s\left(\underline{r}^{\prime}, t\right)$, the source gives

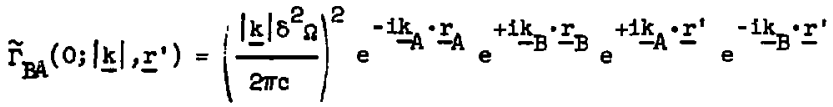

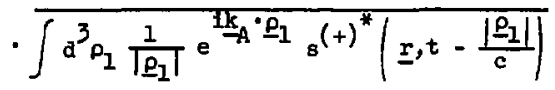

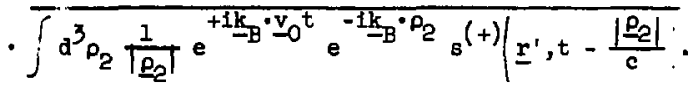

Using again the result of Appendix B.2, we have,

$$
\tilde{\Gamma}_{B A}\left(0 ;|\underline{k}| \underline{r}^{\prime}\right) \underset{t \rightarrow \infty}{\rightarrow} e^{-1 \underline{k}_{A} \cdot\left(\underline{r}_{A}-\underline{r}^{\prime}\right)} e^{1 \underline{k}_{B} \cdot\left(\underline{r}_{B}-\underline{r}^{\prime}\right)}\left(\delta^{2} \Omega\right)^{2}
$$

$$
\text { - } \overline{\left[s^{(+)^{*}}\left(\underline{r}^{\prime},|\underline{k}| c\right) e^{+1 \underline{k}_{B}{ }^{*} \underline{V}_{0} t}{ }_{s}^{(+)}(\underline{\underline{r}}|\underline{\underline{k}}| c)\right]} \text {. }
$$

If the velocity $v_{0}$ is not too large, the factor $\theta^{-1 k \cdot{ }^{*} v_{0} t}$ will

be nearly constant over the interval of the time average. In such cases, the above result becomes, 


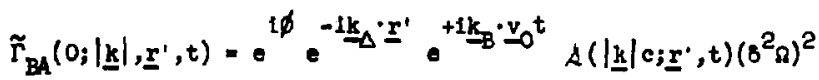

where $\phi, \underline{k}_{\Delta}$, and $\left\langle\left(|\underline{k}| c ; \underline{x}^{\prime}, t\right)\right.$ are defined as in Chapter II.A.3. If the light is so nearly monochromatic that $\phi$ and $k_{\Delta}$ way be treeted as constents, as was done before, the output of the systeen 18

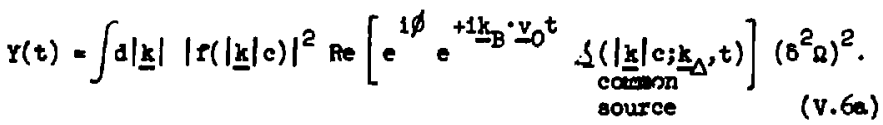

So the spatial resolution 18 unchanged. The system still observes the $k_{\Delta}$ component of the distribution of conmon sources, but the introduction of a woving mirror has changed the time dependence of the output. Taking a frequency spectrum of the signal,

$$
\begin{aligned}
& Y(\omega)=\int d t e^{t \cos t} Y(t) \\
& \left.=\int d|\underline{k}||r| \underline{\underline{x}} \mid c\right)\left.\right|^{2} \operatorname{Bo}\left[\theta^{1 \phi}\left(|\underline{\underline{k}}| c_{j} \underline{\underline{k}}_{\Delta^{\prime}} \omega+\underline{\underline{k}}_{B} \cdot \underline{-}_{0}\right)\right]\left(\delta^{2} \Omega\right)^{2} \text {. }
\end{aligned}
$$

So the tiwe dependence of the observation has been "heterodyned" in frequency. The a frequency component of the output correoponds to the $\omega+b_{B} \cdot v_{0}$ trequency component of the $k_{\Delta}$ wave vector compant of the source distribution. The addition of a moving arror has ahifted the observed rrequency range frow near de to on equel band around $\mathrm{k}_{\mathrm{B}} \cdot \mathrm{V}_{0}$, thus permitting observation of higher frequency prenomone.

It should be remembered, however, that the factor $e^{1 k_{B} \cdot v_{0} t}$ wes treated as constant in the time average which defined the 11stht 1atensity. Such an aseumption 18, in fact, required for 
consistency, wecause it was assuned that the light frow each point $\underline{Y}$ ' wes coherent, that the spectrum was so narrow that the disferences in optical peth were the for all accepted wavelengths. The requinumint of a narrow spectrum 1s, in 1tself, a low-froquancy restriction. The above result is still signtelcant, since the time depondence of even a narrow spectral feature may be too repld to be esily observed, but to observe still higher trequencies, one wust accept a larger portion of the spectrua and the requirement of coherence must in modified.

\section{B. Correlations Between Light of Different Frequancies}

Before continuing with the gederal axalysis, it seems sppropriate to say a little more about the ways in which these higher frequency correlations could be meesured. The use of a moving airror, or sowo similar device, ${ }^{53} 18$ only one of several possibilities, and a few other options might be mentioned.

One way in which the observation could be heterodyned in froquency 18 by employing a past shutter. If an olectro-opt.1c element or sume other repld gate 54 were placed before the system and oultched at a frequency $\Omega$, the entire observation would then be shifted in frequency by that amount. Th1s can be seen quite edoply. Just Imagino that the plasma wave observed had frequency a. Then the ligbt would oscillate at th1s frequency between beams 1 and 2. If the spectrometer were gated, so that the w1ndow was open only when the light was polarized as 1 , the signal would appear at zero frequency.

Another way in which high-frequency effects could be observed 
Is sugse sted by a more careful examination of the syotem shown In Fig. V-2. There a moving mirsor is used to shift the frequency of the light in beam B, the 11ght in beams A and B is then processed by the sume spectrometer, and the mutual coherence of the two is measured by combining $A$ and $B$ and measuring the intensities of the Intermediate polerizationg-s"Interference patterns 1 and ?". Now the observation of interference is not the only way ir. which thi mutual coberence could be measured. $T \mathrm{Th}$ - mentitude of $\Gamma_{B A}$ could alsu be obtained from an intensity correlation measurement. (see Sect. III.A.) In that case one would not combine beams A and B at all, but would observe the two with separate photetubes and then record the correletion of the two intengities. Mis is owething which could be done with light of different frequencles. The woving mirror in Fib. V-2 does not arfect the 1ntensity of $B$, It only shifts the frequency. One could separate thr. same light without the mirror if the spectrometer were readjusted to the orlginal, unshifted frequency of B. Since A is unaffected, one would ther: require two spectrometers or spectral filters set to different frequencles, wo shown in Fig. v-3. If an intensity correlation measurement vere practical, one could just select one frequency component of beam A and another frequency component of beam $B$ and then observe a correlation of the two intengities. However, care should be taken to determine the usable aperture, Which might be severely limitad by requirements of coherence, and a more detailed analysis of the significance of an intensity correlation measurement--which is really beyond the domain of the 


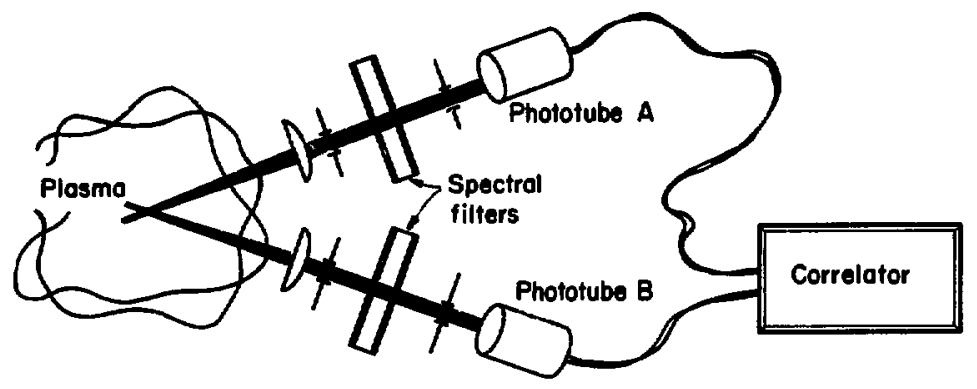

XEL733-2513

Fig. V-3. The use of Intensity correlations to observe highfrequency phencuena. 
present study--should also be done before attempting this type of experiment.

In whetever way the experiment is done, the object is to mreasure the coherence between two beams of light of different Prequencies. This concer ${ }^{t}$ itself is not new. As hes been noted by several authors, ${ }^{55}$ there is nothing inconsistent alout the 1dea of coherence between light waves of alfferent frequencles. one can always imagine Doppler shifting the frequency and then comparing phsses, and any of the other techniques just mentioned would also serve to introduce the same Idea.

In a formal analysis, it is only necessary to fnclude a time dependence in the correlation function. Thus where we had before

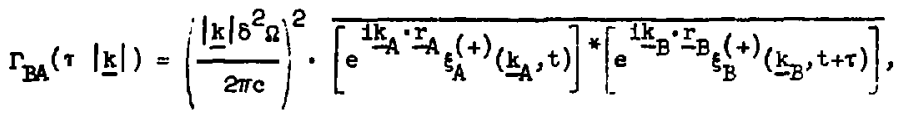
we should consider now

$$
\begin{aligned}
& r_{B A}\left(\tau,\left|\underline{k}_{A}\right|,\left|\underline{k}_{B}\right|\right)=\left(\frac{\left|\underline{k}_{A}\right| \delta^{2} \Omega_{A}}{2 \pi c}\right)\left(\frac{\left|\underline{k}_{B}\right| \delta^{2} \Omega_{B}}{2 \pi c}\right) \\
& \text { - } \overline{\left.e^{1 k_{A} \cdot \underline{r}_{A}(+)}\left(\underline{k}_{A}, t\right)\right]^{*} e^{1 \sim \omega t}\left[e^{1 k_{B} \cdot \underline{x}_{B_{B}}(+)}\left(k_{B}, t+\tau\right)\right]}
\end{aligned}
$$

where the frequency difference,

$$
\Delta \omega=\left|\underline{k}_{B}\right| c-\left|\underline{k}_{A}\right| c .
$$

To afmplify the following equations, it is assumed here that 
the light accepted through each beam is quasi-monochromatic. Then only one value of $\left|\underline{k}_{A}\right|$ and one value of $\left|\underline{k}_{B}\right|$ need by considered. The two frequencles, however, are not equal, and hence the total spectrum is not narrow. Indeed, this is required for the observation of high-frequency phenomena, since the frequencies of the phenomens observed cannot exceed the bandwidth of the light.

In all of our preceding calculations, light from different polnts $\underline{Y}^{\prime}$ withln the plasma was considered incoherent, whlle light from the same source point was assumed to be completely coherent and, in effect, was treatea as monochromatic. That assumption clearly is not valid in this high-frequency analysis, since light fram each point is far from monochromatic, and hence our torwer, siople pleture of the source is not appropriate. The easlest way to generalize the picture is to represent the plasma as a set of moving sources. Bach source may still be considered monochromatic In its own frame of reference, but since the source is moving, the emitted light will be Doppler shifted to produce a broadened spectrum. One could do this by replacing the source density (in $\underline{r}$-space) by a distribution function (dependent also on velocity), but for dmplicity we shall consider onl; a discrete set of sources:

$$
s(\underline{r}, t)=\sum_{j} \cos \omega_{j} t+\phi_{j}(t)\left[\delta \underline{r}-\underline{r}_{j}(t)\right] .
$$

Here the phases $\varphi_{j}(t)$ are independent, so the different sources are all incoherent. Bence the mutual coherence $\Gamma_{B A}$ may be written as a sum of contributions from the separate sources. Making this assumption, and then evaluating $\Gamma_{\mathrm{BA}}$ gives 
$-163-$

$$
r_{B A}\left(0,\left|\underline{k}_{A}\right|,\left|\underline{k}_{B}\right|\right)=-8^{2} a_{A} \delta^{2} \Omega_{B} e^{-1 k_{A} \cdot \underline{r}_{A}} e^{+1 k_{B} \cdot \underline{r}_{B}}
$$

$$
\begin{aligned}
& \cdot \sum_{j}^{1} \int_{-\infty}^{t} d \tau_{1}\left[e^{1 / k_{A} \mid c\left(t-\tau_{1}\right)}-e^{-1 / k_{A} \mid c\left(t-\tau_{1}\right)}\right] e^{1 k_{A} \cdot \underline{r}_{j}\left(\tau_{1}\right)}
\end{aligned}
$$

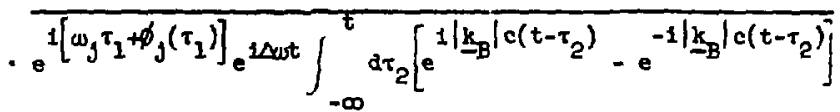

$$
\begin{aligned}
& \text { - } e^{-1 \underline{k}_{B} \cdot \underline{x}_{j}\left(\tau_{2}\right)} e^{-1\left[\omega_{j} \tau_{2}+\phi_{j}\left(\tau_{2}\right)\right]} \text {. }
\end{aligned}
$$

Since each source is nearly monochromatic, the phases $\phi_{\mathrm{J}}$ are all slowly varying quantities. More precisely, they vary only at frequencies less than the optical bandwidth. But this implies that these phases may be taken constant in the $\tau_{1}$ and $\tau_{2}$ integrals whish define the spectre, since these integrals are really taken only over the preceding inverse bandwidth interval. Hence it is admissible to replace $\phi_{j}\left(\tau_{1}\right)$ and $\phi_{j}\left(\tau_{2}\right)$ by $\phi_{j}(t)$.

It also greatly simplifies the result to assume that all the sources move without acceleration and set

$$
\underline{r}_{j}(t)=\underline{r}_{j 0}+\underline{v}_{j} t .
$$

Under these assumptions the above result reduces to

$$
\begin{gathered}
r_{B A}\left(0,\left|\underline{x}_{A}\right|,\left|\underline{k}_{B}\right|\right)=8^{2} a_{A} 0^{2} \Omega_{B} e^{-1 \underline{k}_{A} \cdot \underline{x}_{A}} e^{+1 \underline{k}_{B} \cdot \underline{r}_{B}} \cdot j_{j} e^{-1\left(\underline{k}_{B}-k_{A}\right) \cdot \underline{x}_{j 0}} \\
\cdot \delta\left(\omega_{j}+\underline{k}_{B} \cdot \underline{v}_{A}-\left|\underline{k}_{B}\right| c\right) 8\left(\omega_{j}+\underline{k}_{A} \cdot \underline{v}_{j}-\left|\underline{k}_{A}\right| c\right) .
\end{gathered}
$$


Bx.ept for normalization, th1s expression just denotes the $\underline{k}_{\Delta}=\underline{k}_{\mathbb{P}}-\underline{k}_{A}$ component of the distribution of those sourcer which have erequency and veloutty such that

$$
\begin{aligned}
& w_{j}+\underline{k}_{-B} \cdot \underline{y}_{j}=\left|\underline{k}_{B}\right| c \\
& \omega_{j}+\underline{k}_{A} \cdot \underline{y}_{j}=\left|\underline{k}_{A}\right| c .
\end{aligned}
$$

Putting these relations in a more convenient form, we have the two conditions

$$
\begin{aligned}
& \left(\underline{k}_{B}-\underline{k}_{A}\right) \cdot \underline{v}_{j}=\left|\underline{k}_{B}\right| c-\left|\underline{k}_{A}\right| c=\Delta w \\
& \omega_{j}+\frac{1}{2}\left(\underline{k}_{A}+\underline{k}_{B}\right) \cdot \underline{v}_{g}=\frac{1}{2}\left(\left|\underline{k}_{A}\right| c+\left|\underline{k}_{B}\right| c\right) .
\end{aligned}
$$

The first condition, Eq. (V.10a), just restates our earlier result, Eq. (Y.2), which was obtained frow a much more elementary argument. The point is the same: The frequency alfference between light emitted in the $\hat{k}_{A}$ and $\hat{k}_{B}$ directions depends upon that compowent of the source velocity which is perallel to $\mathrm{k}_{\Delta}$. Hence, by corrolating light of different frequencies emitted in these two ds rections, one selects one velue of this source relocity component. Only sources with such motion can contribute to the stgral.

The second condition, Iq. (V.10b), Just gives the usual effect of Doppler brosdening, as 1s seen in conventlonal spectroscopy. Bore it is the $\left(\underline{\underline{k}}_{A}+\underline{k}_{B}\right)$ velocity component wh1ch changes the efrarent erequency of the source.

What this calculation shows is that the effect of Doppler 
brosdening could be deduced from correlation measurements--even with a spectrum further brodened by anotber mechan1 sm. We assumed that each source was monochromatic in its frame of reference, but did not assume that all the frequencles $\omega_{f}$ were equal. These frequencles could differ and this difference would produce a broadened spectrum. If such broadening concealed the Doppler shift, the source velocities could not be measured by conventional spectroscopy .

This is sometbing which does happen in a plesma. In any plassp there are electric flelds, fluctuating fields which through the wechanism of the Stark effect can broaden spectral lines. (we mean here the quar1-static, or Holtsmark type of Stark broadenIng, not collisional broadening which spreads the spectrum of the light enitted Prow each atom.) Th1s brosdening can exceed the Doppler broadening und conceal the Doppler line shepe in the spectrum. Then the Doppler broadening cannot be seen--at least not withnut some kind of unfolding.

In such cases, novertheless, a detafled record of the source velocities is still present in the radiation. As the preceding calculation shows, such informstion could be found from observetion of the phase relations--of the correlations--between different eroquency components of light emitted in directions $\hat{k}_{A}$ and $\hat{k}_{B}$. Whon differences in trequency are allowed, a two-bean spectroscople observation could provide the distribution, not just in space, or $\underline{k}$, tut also in velocity, of the sources of each feature in the spectrum. 
Th1s result, of course, suggests some interesting experiments. Moreover, the analysis which we have done 1 s only a beginning, for the effect of other opt1cal arrangements and of other correlation messurements remeins to be determined. We shall not pursue these queations further here; that would be a separate project. The foregoing discussion 1llustrates that an extension of the metbod to high frequencies 16 possible. An exploration of this possibility could be the subject of a later study. 


\section{CONCLUSIONS AID SUGGESTIONS}

\section{A. A Summary of Results}

This project began with the realization that spatially local12ed information about particle correlations--information of the type provided by a scattering experiment--is in fact present in the light emitted by a plasma. The inftial objectives were to prove this fact and then to demonstrate that such information could be obtained with a practical, convenient diagnostic instrument. The facts about pt se measurements could probably have been shown with a simple two-beam system, but since the two-beam arrangement 1s so Inefflclent, the development of a more efficient design was crucial to the question of practicality.

The multiple-beam system which was ultimately constr ded Involved two design pl sples: the use of polarizetion interference and the use of many independently-cullimated pairs of beams. our particuler system could, of course, be improved, but the se two techniques should be worth considering 1. the design of any such device.

The particular syotem described here can be clafmed to have served Its intended purpose: The optical tests verifled the theory of the design and the plasma observations showed that such a system ean be used for plane diagnostice.

Regerling the experimental work, three comments seem worth making in conclusion. Firatly, as wo have already noted, the optical bystem vhich vas used was made from components of quite ordinary quality. The lenses were ell single elements; the patterns 
shown in Sect. IV.A were made using sheets of plastic polarizing materlal. Furthermore, the plasma was observed through a lucite vacuum window which was curtainly not of high optical quality. It was originally thought that this window would have to be replaced, but before doing so the lucite was placed in the optical train of a test system like those described in sect. IV.A. A pattern like that shown in the lower fram of Fig. IV-14 was produced, and the presence of the lucite seemed to have no effect whatsoever. The reason is that the lateral displacement between interfering beams was so amall that the lucite (and the other elements) did not bave to be very flat for the optical paths to be equal. After th1s discovery, the lucite window was put back in place and all the plasma obrervations were made right through 1 t.

The second point is that the total solld angle subtended in our plasma observations was extremely small. The apterture tas $0.64 \mathrm{cos}$ square at $61 \mathrm{~cm}$ frum the plesma. This amounts to $1.1 \times 10^{-4}$ sterradians, or less than one-thousandth of one percent of the whole solid angle. Thus, although our system was more efficient than a two-beam setilp, it was still extremely weak in terms of the total light avallable. The destgn which we used could be extended to a system with s much larger aperture. $r$ With wore expense, but with the save spproach, one could obtain orders of magntude wore light. (Also, the piesma used here was not particularly luminous. An arc discharge, for example, would be much brighter than the beam-plasma system on which these measurements waro meo.) 
Finally, it should be remembered that a multiple-beam system of the design used here requires that all the light transmitted by the collimator be accepted by the monochormator. Ih1s means that the entrance slit cannot te too small, and hence that the spectral resolution 1s restricted. (In princlple, of course, the resolution could be improved by using a more dispersive grating.) In our case th1s was not 1mportant, since the observed spectral line was stronger than any nearby feature, but in planning such e system, one should make sure that the Intended phase and frequency measurements are compatible, and that the coherence length of the accented light will exceed any difference between the lengths of the paths of the $A$ and $B$ components of the $11 \mathrm{gl} \cdot$

In the course of studying these systems, it has become increasingly apparent that this prob] em involves much more than just the spectroscopic analogue of a light scattering experiment. The Gpetroscopic problem is much broader to begin with, because the varlety of sources 1a much greater. Scattering is due mainly to the plasma electrons, but the emission spectrum includes light Prom many differert groups of plasm particles. But beyond this difference, the apectroscopic problem is more diverse because the number of possfble optical systems is much greater. In principle, one could construct multiple-beam spectrometers which would observe many different components of a light source distribution. The selection of one $\underline{k}$ component of the sources vithin a local region 1s only one among many possibilities.

To be better able to discuse the problem in some generelity, 
two different mathematical descriptions of th1s type of optical eystem have been mesented. Revlewing briefly, the flrst approach was based upon spat1al Fourier trangforms. In a preliminary analyB1s of scattering (In Sec. I.C) 1t was shown that the light of one wavelength emitted in a given direction could be expressed in terms of the positive frequency portion of one $\underline{k}$ component of the radiation from within the observed region. Th1s description was then used (In Sect. II.A.3) to anslyze a two-beam spectroscopic system. Assuming that the light from each point was coherent but that light fram lifferent points was incor rent, it was shown how the measured correlation between Ilght in the two beams could be written as an integral over source points and wave numbers [Eq. (II.13)]

$$
Y(t)=\int_{\substack{\text { cormon } \\ \text { source volume })}}^{d^{3} r^{\prime}} \int_{0}^{\infty} d|\underline{k}||f(|\underline{k}| c)|^{2} \operatorname{Re} \Gamma_{B A}\left(0 ;|\underline{k}|, \underline{r}^{\prime}\right) \text {. }
$$

The mutusl coberence, $\Gamma_{\mathrm{BA}}\left(0 ;|\underline{k}|, \underline{\underline{r}}^{\prime}\right)$ was then expressed in tecens of the sourec a1stribution:

$$
r_{B A}\left(0 ;|\underline{k}|, \underline{r}^{\prime}\right)_{t \rightarrow \infty} e^{1 \phi-1 \underline{k} \Delta^{-\underline{r}^{\prime}}} 0^{2}\left(\delta^{2} a\right)^{2} \underline{i}\left(|\underline{k}| c ; \underline{r}^{\prime}\right)
$$

Bore $\&\left(|\underline{k}| c_{i} \underline{y}^{\prime}\right)$ the spectrum of the light emitted by sources noer $\underline{r}^{\prime}$ and $e^{-1 k} \Delta^{\prime} \underline{\underline{r}}^{\prime}$ is a complex pbose factor which appears beweuse the lengths of the optical paths depend upon the source position. Because of this factor, the integral over $\underline{r}^{\prime}$ is just

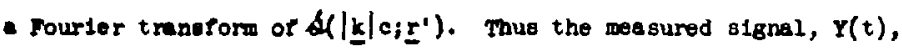
Io found to we dwe to one Pourier component of the source distri- 
bution.

This analysis was later extended to high-frequency effects (in Chapter V) and to higher order correlations (in Sect. II.C). The second description presented (in Sect. II.B.2) was not Iimited to narrow pencils of rays. Instead, it was assumed that the accepted IIght (Initlally polarized) could be divided into two orthogonally polarized components whlch would be treated separately by the optical system. The system was assumed to be linear, so that each effect could be described by a transfer function i Eq. II. 23) \}

$$
\xi_{A, B}\left(\underline{r}^{\prime \prime}, \omega ; \underline{r}^{\prime}\right)=\varnothing_{A, B}\left(\underline{r}^{\prime \prime}, \underline{r}^{\prime}, \infty\right) B\left(\underline{r}^{\prime} \omega\right)
$$

Here $\underline{r}^{\prime}$ denotes points within the source volume and $\underline{\underline{r}}$ denotes points across the entrance to a monochromator. $Y(t)$, the output fram the gystem was then shown to be given by an integral over $\underline{r}^{\prime \prime}$ of the coherence between the waves $\xi_{A}$ and $\xi_{B}$. Assuming the light iron different points to be completely incoberent, we obtained the result, $[\mathrm{Eq} \cdot(\mathrm{II} \cdot 24)]$

$$
Y(t)=\int d^{3} \underline{r}^{\prime} \int d \omega|f(\omega)|^{2} T\left(\underline{r}^{\prime}, \omega\right) \mathcal{L}\left(\omega ; \underline{r}^{\prime} t\right)
$$

whore

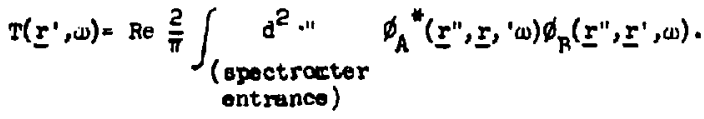

Thus the runction $T\left(I^{\prime}, w\right)$ describes the efiect of the correlation moesureat. S1nce many trencfer runctione $\phi_{A, B}\left(\underline{r}^{\prime \prime}, \underline{r}^{\prime}, \omega\right)$ cen 
obvlously be produced, a great varlety of functions $T\left(\underline{r}^{\prime}, \omega\right)$ could be gene,ated.

Several spectroscoplc systems were analyzed (In Sect. II.B.3) by this technique and the conclusions wore later conflrmed by opt1cal testa done in the course of the laboratory wcrk (described in Sect. IV.A).

Finally, the effect of photon nolse in a model systein was analyzed in chepter III. There $1 \mathrm{t}$ wes concluded that if the observed component of the source density oscllleted at a frequency $\omega_{0}$, and if a spectrm analyzer were used to select $\mathrm{a}$ band around $\omega_{0}$, the effect should be measurable if [inequality(III.8)],

$$
\mathrm{P} \sqrt{\mathrm{T} T}>\sqrt{8} \frac{\mathrm{Q}}{\mathrm{P}}
$$

Here $P$ and $Q$ are the signal and background photocount rates, $T$ is the analyzer inverse banduldth, and $T$ is the time of observation. In our plasma observations, phase-sensitive detection was used to reduce the nolse, so the above result dues not apply directly to the rata, but it does imply that the algnal could not bave be an observed without the lock-In amplifier. When measurements of plaswa luminosity (see Appendix D) are scaled to our exporimental conditions, the total photon counting rate deduced Is roughly $10^{6} / \mathrm{sec}$. The signel level was one-tenth of one percent of background (see sect. $\mathrm{N} . \mathrm{b}$ ) so $Q=10^{6}$ and $P=10^{3}$. The inveree banduldth $T$ yas roubhly $10^{-5}$ bec and the observation time wes about 10 aec. Since these numburs do not satisfy the above criterion, It appers that the effective aperture of the system 
vould have to be increased before an umodulated signal of this level could be observed.

The theoretical analysis developed here is clearly more than ves needed to explein our messurements, but the general formalism should be useful if this work is to be carrled any further. If the project were to be continued, it would not be necessary to go on with all of the toples which we have mentloned. By discussing a number of related problems, it was hoped to provide here an overview of some of the broaier implications of these correlation measurements. But now it should be possible to concentrate on one or a few aspects of the problem without losing sight of the whole pleture. Such a greater specialization should perpit reasonably repld progress from this point.

\section{B. Extensions of This Work}

There is no ehortage of alrections in wh1ch this work could be continued. One obvious next step is to now use such a system for detailed observatione of a plasma. Only a few simple features of the plasme used in this work were considered. We bave concentreted on the optics of the spectroscopic system. The other half (or perbaps the other ninety percent) of the problem is to see what can be learned with such a system when it is used to observe a placan. The aveilability of this nou tool should permit a verloty of interesting experisents.

The multiple-beam spetroscopic systom de seribed bero could be improved in severel ways. One could use a system w1th a larger eperture, or with brttor apectral rosolution, or with better 
electronics to reduce the nolse.

As noted in the preceding section, the photon noise analyals presented in Chspter III hes not been tested. In any further study of these spectrometers, the level of noise, including photon noise, should certainly be examined in more detail.

The range of possible multiple-beam systems--1.e., or possible transmission functions $T\left(\underline{x}^{\prime}, \omega\right)$ bas only begun to be explored. One could construct and test a much greater variety of systems, and the analysis presented here could also be continued. Our calculations (in Sect. II.B.3) were limited by several simplifying assumptions, including a restriction to points near the focal plane of the system. The calculation of the functions $\phi_{A}, \phi_{B}$, and $T$ for systerns of this type could be done more completely. without such restrictions as were used here. This is a problem which seems well suited for the use of some numerical analysis, which we bave not emplcyed at all.

Also, in the general snalysis, it would be valuable to knuw what type of systems are rossible in principle. Given azy desired $T\left(\underline{x}^{\prime}, w\right)$, couló on dealga a system which would produce $1 t$, or are the besic metbentical restrictione on the transfer functions $\phi_{A, B}$ and the trensonission runction $T$ which can be generated? One property of the light sources which we have not discussed at all is engular coborence. All of the analyses done here assumed en 1cotropic source. This is acceptable when the range of angles actually uced Is umall, but if the observations were extended over lerger ancles, tbe effect of the source rediation pattern would 
have to be included. This would give an added complication in the theory, but it would also provide a way in which radiation patterns could be measured. A jeasurement of single-source radiation patterns would be of interest, for exs ' ?e, in observing bremsstrublung or cyclotron radiation where the pattcrns depend upon particle energies.

Another property which could also be observed is the lateral coherence of the source. We have assumed throighout that any difference between the lengths of the interfering veams was less than the coherence length of the accepted light. Ore might, however, want to deliberately introduce a path length difference in order to weasure the coherence length, thereby obtaining informetion Bbout the spectmom. Th1s, in 1tself, is nothing new. The point 1s simply that when one had a multiple-beam aystem it would be relatively easy to add a path length difference. Th1s sbould be particularly useful since muliple-beam systen would otherwise be limited in spectral resolution (see Sect. VI.A above). The addition of a coherence length measurement could be a conventent vay to avold exectly thls restriction.

Another possible extension is suggested by the origins of the multiple-beam technique. The development began with an analogy with scattering, where it was argued that one could obtain similar information from measurements of correlations in the light emitted by an incoherent source. Hiaving used both methods, one wight yonder whether it would not be of interest to combine the two tech. aiques, using a multiple-beem system to observe scattered light. 
To understand such a measurement, one would have to do a separate analysis, since the assumption of an incoherent source is not valid in scattering, but a part of the answer can be seen already. In scattering, the amplitude of the light of wave vector $\underline{k}_{s}$ is due to one Fourier component, $n_{e}\left(\underline{k}_{\Delta}, \omega_{\Delta}\right)$, of the electron density. (See sect. T.C.) Such fluctuations tyvically are due to plasma waves. Thus if a two-beam system were used to measure the coherence between two components of the scattered light, the result would be a measure of the correlation between two waves in the plasma, s result, which would in turn give information about higher order correlations between particles. Clearly, both the optics and the photocount statistics should be analyzed with care before attempting any such experiment. Success woulā probably require a very strong Incident light beaw, but there may well be situations (for example, in laser-produced plasma experiments) where correlations in the scattered light could be observed.

In our plasme observeitions, the light used was a neutral belium emission line. This was conventent because, in this weakiy Ionized ges, the strongest neutral Iine kas narrow enough to provice coherence and strung enough to give a high intensity. In more fully ionized gases, however, line radiation would be weaiser or even lacking altogether. 'fo observe such a plesme, one would have to make use of the continuum. With our system this would be more difficult, because the spectral resolution would become critical and beceuse the amount of light avaflable in any narrow band would be IImlted. Thus it would be of value to see whether the 
optical bend of the system could te increased. In our system a broad spectrum could not be used, even if all the paths through the apparatus were made exuctly equal, because the paths of the $A$ and $B$ components within the plasme would etill difier by more than the coherence length of the light. What this reans is that light of different wovelengths would have different phase changes and hence would produce different interference patterns--i.e., that the observed source density component $T\left(\underline{r}^{\prime}, \omega\right)$ would in fact be a function of $\omega$. If too broad a spectrum were used the effect would wash out. This suggests an answer: If $T\left(\underline{r}^{\prime},(\omega)\right.$ could be made independent of $\omega$. then 1 t would be possible to use a broad optical band.

In our syatem the observed source density component had a wavelength $\left(\lambda f_{1} / d\right)$, where $\lambda$ was the optical wavelength, $f_{1}$ was the focel length of the first lens, and d was the displacement of one of the Interfering beams. If the displacement a were not a constant, but were snstead proportional to wavelength, then the whole expression would be wavelength independent. This would happen If the calcite rhomb were replaced by an element which gave an offset proportional to the wavelength of the light. Interestingly enough, our system already contained a device which gives a wavelengih dependent displacement--namely, the grating spectrometer. If the entrarce and exit apertures were made Iarge enough, one polarization component could be put through the sjectrometer and then recombined with the other component to give a wavelengthindependent interfelence effect. Such a change would greatly 
extend the utility of multiple-beam systems.

The posalbility of making the device broad band is also interesting conceptually. Up to this point, we have considered a correlation measurement as something additional to a frequency measurement. Physically, our interferometric apparatus was mounted In series with a standard spectroscopic instrument. But now it appears that the tecknlque ca"? 2 be made broad band and could be used to extract useful information from light whose spectrum is flat and uninformative. Th1s possibility clearly shows that what we are dealing with here is really a separate aspect of the light-one which mav have littie or nothing to do with the frequency gpectrum.

\section{Final Comments}

In cxmparison with a conventional spectrometer, a multiplebeam system has the advantage of providing spaticl resolution. The output from a multiple-beam system is a local measurement, not just an average along a line of sight. However, a multiple-beam spectroscopic system is certainly not the only optical device which hes such an effest. An ordinery camera also provides depth perception. By noting which objects in a photograph are in focus and which are out of focus one can tell something about distances along the line of sight. In closing our discussion here it might be of interest to examine the relation retween the depth of fleld provided by a camera and the spatial reolution of our spectroscopic system.

The depth of field of a camera is the range of distances over 
which objects aro in focus, 1.e., the distences over which a small source is imaged to a small spot on the fllm. According to physical optics, an image is simply a diffraction maximum, a sharp peak In Intensity produced by Interference between the Hurgens wavelets of the 1 fght hehind the lens. Thus a statement about the sharpness of an lmage is really a statement about the amplitude and widtis of a diffraction maximum.

To see how this effect could be simplifier, one wight try to reduce the number of interfering waves by masking off portions of a camera lens. If the lens were masked down to one swall aperture, the result would be a pinhole camera in which depth perception would be lost. There the light coming through the aperture would contain information about direction (the slope of the wavefronts), but information about source distance (the curvature of the wavefrorts) would be lost. To avold this one might try masking off most of the lens, but leaving several small apertures. Then the I'-ght coming through each hole would have a drection and the different directions combined would imply the listance of the Bource. The result on the film would no longer be a clear inage, but would be a set of interference fringes. Most simply, one could leave just two apertures in the lens. Then if the source were away from the focus of the system the two beams of light would strike the film at different points, but if the source were in the focal plane, the two beams would intersect on the film to give a set of interference fringes, a result which thus would conotitute the most rudimentery precursor of an image. But now we 
are right back to the two-beam system with which this whole aiscussion started:

It is important to realize that in order to detect any property like the sharpness of an inage, ore has to ubserve at least two light intensities. With one light intensity value, one has no way of knowing whether the source is in focus or not, but two intensities give an additional piece of information--namely whether they are equal or different. From that one can say something about the sharpness of an interference pattern, 3 statement which is simflar in kind to statements about the sharpness of images. In this sense, the "multiple-beams" of importance in our system were not so much the observed beams $A$ and $B$, but rather the two measured light intensfties, 1 and 2.

one can think of a scale, an ordering of optical techniques according to the number of intensities observed. At one end of the scale are spectroscopic methods in which, at least at each wavelength, only a single light intensity is observed. At the other end of the scale are photographic methods in which many light intensity values are recorded on the film. The subject of the present study l_ss between these two extremes. We have shown that by taking the single step from one to two intensities, one can obtain new typeis of information. Two is a convenient number of interisities to uise, because as we have seer., it is then only necessary to consider the single difference signa.l $Y(t)$. Two is a convenier: number also because such a system can te simply constructed out of polarizing optical components. 
It might be objected that the measurement could have been made with beam 1 alone, by observing features in the frequency spectrum of the output $I_{1}(t)$. But in that case, one would still be taking the dffference between two light intensities, namely the intensities seen by the same phototube at different times. Botb types of intensity differencing are used by the human eye and by other natural optical systems. Sharp spots or edges In an image are tmmediately apparent to a person, and any motion of Iight patterns is noticed at once. Un the other hand, the overall luminosity can change by orders of magnitude and the eye will adjust quite completely to keep the signal the same. of course, one can make too much of any such comparison, tut the results of the evolutionary process do seem to suggest that differences in light intensity are much more interesting than total light levels.

A comperison with a camera, and with the eye, is also useful because of what it shows about the concept of phese. One doec not normally think of the human eye as making measurements of mutwal coherence, but ô course it does. An image is a diffraction maximun and any Interference or diffraction effect depends upon the coherence of the I1ght. Thus "phase" is a much broader categury than one might have thought.

The term cones originally from the simple mathematical description of a nearly sinusoidal quantity:

$$
a(t) \cdot \cos [\omega t+\phi(t) \cdot
$$


where

$$
\begin{aligned}
& I_{0}=\frac{c}{4 \pi} 2\left|\underline{E}_{1}^{(+)}(\underline{r}, t)\right|^{2}=\frac{c}{4 \pi} e\left|\frac{E_{0}}{2}\right|^{2}=\frac{c}{8 \pi}\left|E_{0}\right|^{2}, \\
& n_{e 0} \text { is the meen electron density, } \\
& \sigma_{T}=\left|\frac{e^{2}}{m c^{2}}\right|^{2} \frac{\left|E_{0}\right|^{2}}{\left|E_{0}\right|^{2}}
\end{aligned}
$$

Is the differential Thompson scattering cross section, and

$$
s\left(\underline{k}_{\Delta}, \omega_{\Delta}\right)=\frac{1}{2 \pi m_{e 0}}\left|\ln _{e}\left(k_{\Delta}, \omega_{\Delta} ; t\right)\right|^{2}
$$

Is celled the "dynamic form fector."

In a scattering experiment, one records a spectrum of the light scattered into some direction $\hat{k}_{s}$. The reiative variation In optical wavelength is usually negligible, so the scattering is alI due to Pluctuations of one wevelength, $2 \pi|k|^{-I}$. Th1s is customar1ly related to the plasma Debye length $\lambda_{\mathrm{D}}$ by a "scatterIne parrmeter",

$$
\alpha \equiv \frac{J}{\left|k_{\Delta}\right| \lambda_{D}}
$$

The spectrum of scatterea light then provides a frequency spectrum of the $k_{\Delta}$ corpponent of $n_{e}$. By the Wlener-Khintchlne theorer, ${ }^{2 l}$

$$
\frac{1}{2 \pi}\left|n_{e}\left(k_{\Delta}, w\right)\right|^{2}=\int d \tau c^{L \omega r} \overline{n_{e}^{*}\left(k_{\Delta}, t\right) n_{e}\left(\underline{k}_{\Delta}, t+\tau\right)},
$$

this 1s equivalent to a measure of the time correlation function, 


\section{ACKNOWTEDGMENTS}

It is a pleasure to acknowedge the help and encouragement which Prof. Wulf B. Kunkel and Dr. William S. Cooper have provided through the course of this work. Special thanks are due to Margaret R. Thomas for valuable advice on format and for her skill and patience in typing several versions of the manuscript. The author is indebted to Elliott B. Hewitt and to James E. Galvin for help with the apparatus, to Vincent J. Honey for ald with the electronics, and to the mechanical shop of Hurlsn A. Hughes for fabrication of several elements of the system. Thanks are also due prof. Sumner P. Davis and Prof. Franklin A. Robben for several helpful discussions, and to Barton D. Billard for his assistance in conducting the experiments.

This work was perforned under the auspices of the U. S. Atomic Energy Commission. 


\section{APPENDICES}

\section{A. Holography, Spectroscopy, and scattering}

In introducing the concept of multiple-beam spectroscopy, we first reviewed the standard technique of laser light scattering and then proposed 1ts spectroscopic analogue, two-beam spectroscopy. What scattering and our two-beam system have in cormon is a dependence upon phase relations, a dependence which leacis to reauits which are inexplicalole in geometrical optics terms. Now there exists also enother class of optical techniques of which the same thing is true. These are the varlous methods of holography, 24,25 whtch have been extensively investigated. There is an interesting connection between several of the different holographic methods and the scattering and spectroscopic systems which we have been considering. In the following appendix (which assumes sawe knowledge of holography) the relation between these different methods is examined briefly.

Holography can be explained in several ways. One explanation, which ig particularly well suited for a compariscn with seattering, is that presented by H. M. Smith in his book on holography. ${ }^{24}$ Considering off-axis holograms (in which the reference wave and the object wave intersect the photographic plate at different angles) be describes the object wave as a superposition of plane wave components. When a hologram is made, each such component interferes with the light in the reference beam (which consists essentially of only one plane weve component) to produce a set of straight, evenly spaced interference fringes on the photosraphic plate. When 
tlie resulting hologram is then rellluminated with the reference keam, each recorded set of fringes acts as a diffraction gating and diffracts the light into a reconstructed wave identical to that plane wave component which produced the fringes when the hologram was made. So the object wave is considered as a superposition of plane waves, or $\underline{k}$ components, and the hologram is then seen as a superposition of diffraction gratings, one for each $\underline{k}$ component of the object wave.

Seen from this point of view, the simllarity to scattering is obvious. In a scattering experiment (see sect. I. C) only a single $\underline{k}$ component of the scattered wave is observed. The intensity of this component gives the emplitude of one Fourier component of the distribution of scatterers.

In this respect, holography is more complete: The hologram is a record of both the amplitude and the phese of every $\underline{k}$ component of the object wave. It thus describes not one, but all of the Fourler components of the object under study. On the other hard, a scattering experiment gives information about the time or frequency dependence of the observed component of the scatterers. one can record a complete frequency spectrum of the scattered IIght which can be complicated and quite useful. In holography, one does not have such information, and the methou will not work at all unless the object studied is precisely stationary or unless the light comes in a pulse so short that object motion is ignorable. St111, in spite of these differences, it is evident that the two methods share at least a substantial portion of a common theory. 
This simflerity suggests that two-bear spectroscopy, which was f1rst introduced by a comparison with scattering, may also have a holographic analogue. This is, in fact, the case. The suggested simlarity is to a very different type of holographic process, incoherent light holography, which can be used to make a hoiogram of a self lumirous or incoherently illuminated object. Incoherent light holography is usually discussed in terms of a somewhat different explanation of hologrephy advanced by Rogers. ${ }^{26}$ Rogers described a hologram not as a superposition of diffraction gratings, but as a superposition of Fresnel zone plates, one for each point of the object. When the hologram is illuminated, ench zone plate acts as a lens to focus light towards the location of the corresponding object point. According to this explenatlun, when coherent light is used to make a hologram, light from each object point interferes with ihe light in tho reference beam to make a fringe pattern which, when photographed, becomes the needed zone plate. Fis this technique is not the only way in which such patterns can be made. There are several other positbilities, some of which apply to incoherently illuminated objects.

One approach is just to use a mask cut as a zone plate. Placed between the object and the film, this mask will cust a set of shadows if the needed form--one for each object point. This, technlque was used hy Jertz and Yourge 27 to make an $x$-ray star camera. In the1r device, each $x$-ray star produced a zone plate on a f13r. When the developed film was then illuminated with coherent visible light it acted as a hologram, focusing the light 
Into an image of the $x$-ray sky. Thus this technique resembled stendard holographic metrods in its reconstruction phase, but the formation of the "hoiogram" wes due only to simple shadow sesting. Mertz, however, then proposed ${ }^{28}$ a purely opt1cal arrangement which also used interference in the meking of the hologram. His auggestion was to use $a$ beam splitter to split light from ar. L..oherently illuminated object into two corponents. These two waves $c$.zd then be focused at two different points above a photographic pilate. Assuming roughly equal lengths of peth (which we" assured in his suggested setup) the contributions to the wo wavefronts from each single object point would interfere to make a set of fringes on the film. The system was arranged to wake this patterm have th: form of a Fresnel zore plate. For an incoherent source, the total illumination of the film would fust be a sum of contributions from the separate source points, and the developed film would be a set of zone plates, just as in coherent light holography.

After Mertz' suggestion, several other jeople ${ }^{29}$ prodosed schemes for making holograms from 11 juit on other radietion from an incoherent source. The several methods outlinad all involved the same idea of dividing the exitted radiation into two component waves, which can then be made to interfere, producing a pattern of fringes which, when photographed, becomes a hologram. Shortly thereafter, several authors ${ }^{30}$ reported succer in making holograns of some simple objects which were incoherently illumireted.

In pracilce, extension of the method to more complicated objects has proved difficult, kecause the superposition of many 
Intensity patterns tends to uniformily expose the film, giving a much lower contrast than is obtained in holograms made with coherent light. Several techniques have been employed to partially alleviate this problem, ${ }^{31}$ but the art of making holograms with Incoherent light apperently has not progressed beyond tile stage of simple demonstrations. keverthel.ess, experiments have clearly verified the theory of the technique: Light from an incoherent source contains sufficient information to construct a hologram.

It is evident from thıs work that incoherent light holography resembles multiple-beam spectroscopy in much the same way that coherent light holography resemble: laser scattering. Like scatierIng, the spectroscopic system observes only one source density component, while \& hologram, recording all the source components, permits reconstruction of a complete ingge of the object. But as In the coherent case, the holographic object must be strictly statIonary, winle the output of a spectroscopic system would follow the time variation of the observed component of the source.

Moreover, the need for contrast is also different in the two techniques. To obtain a photograph of a fringe pattern requires recording the intensity at many difierent points. A spectroscopic sy stem, on the other hand, would measure only two intensities, $I_{1}$ and $I_{2}$, each containing roughly half of all the light accepted. Furtliermore, a small difference between these two should also be more easily distinguished, since the two phototubes can be precisely balanced, us described in Sect. III.B. As explained there, a spectroscopic difference signal far below the level of the hack- 
ground light should still be readily observable--A situation very different from the need for contrast in a fringe patterm recorded for hologrephy.

Nevertheless, the two techniques involve related theories, and thus the demonstrated possibility of making holograms with light from incoherent si drces gives an added proof of the essentia: fact that measuremeats of phase made on such light can sive a complete record of the spatial distribution of the source. 


\section{B. Some Nathematical Detalls}

\section{An Integration Needed in Section I.C}

Tha expression,

$$
\begin{gathered}
\int d^{3} r e^{-1 \underline{k}-s \cdot \underline{r}} \int d^{3} r^{\prime} n_{e}\left(\underline{r}^{\prime}, t-\frac{\left|\underline{r}-\underline{r}^{\prime}\right|}{a}\right)\left[\hat{n} \times\left(\hat{n} \times \underline{E}_{0}\right)\right] \\
\cdot \frac{1}{\left|\underline{r}-\underline{r}^{\prime}\right|} \cos \left[\underline{k}_{1} \cdot \underline{r}^{\prime}-\infty_{1}\left(t-\frac{\left|\underline{r}-\underline{r}^{\prime}\right|}{c}\right)\right]
\end{gathered}
$$

occurs in Eq. (I.6). If one defines $\underline{r}^{\prime \prime} \equiv \underline{r}-\underline{r}^{\prime}$ (so $\hat{n}=\hat{\mathbf{r}}^{\prime \prime}$ ), then (BI) becomes

$$
\begin{aligned}
& \int d^{3} r^{\prime} e^{-i \underline{k} \underline{s}_{s} \cdot \underline{r}^{\prime}} \int d^{3} r^{\prime \prime} \frac{1}{\left|\underline{r}^{\prime \prime}\right|} n_{e}\left(\underline{r}^{\prime}, t-\frac{\left|\underline{r}^{\prime \prime}\right|}{c} ! \mid r^{\prime \prime} \times\left(\hat{\mathbf{r}}^{\prime \prime} \times \underline{E}_{0}\right)\right] e^{-\underline{\underline{k}} \underline{s}^{\prime} \underline{\underline{r}}^{\prime \prime}} \\
& \cdot \frac{1}{2}\left[e^{\underline{1} \underline{k}_{i} \cdot \underline{r}^{\prime}} e^{-1 \omega_{1}\left(t-\left|\underline{r}^{\prime \prime}\right| / c\right)}+e^{-\underline{i} \underline{k}_{i} \cdot \underline{\underline{x}}^{\prime}} e^{+1 \omega_{1}\left(t-\left|\underline{\underline{\prime}}^{\prime \prime}\right| / c\right)}\right]
\end{aligned}
$$

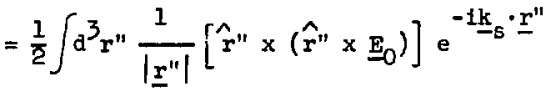

$$
\begin{aligned}
& \cdot\left[e^{-i \omega_{1}\left(t-\left|\underline{r}^{\prime \prime}\right| / c\right)} n_{e}\left(\underline{k}_{s}-\underline{k}_{1}, t-\frac{\left|\underline{r}^{\prime \prime}\right|}{c}\right)\right. \\
& \left.+e^{+1 \omega_{1}\left(t-\left|\underline{r}^{\prime \prime}\right| / c\right.} n_{e}\left(\underline{k}_{E}+\underline{k}_{1}, t-\frac{|\underline{\underline{I}}|}{c}\right)\right] \text {. }
\end{aligned}
$$

Here

$$
n_{e}(\underline{k}, t) \equiv \int d^{3} r e^{-\underline{1 k} \cdot \underline{x}} n_{e}(\underline{r}, t)
$$

1s the spatial Fourier transform of $n_{\theta}$. It is conventent to perform the $\underline{\mathbf{r}}^{\prime \prime}$ Integration in polar coordinates. Replacing $\int \mathrm{d}^{3} \mathbf{r}^{\prime \prime}$ 
-191-

by $\int d\left|\underline{x}^{\prime \prime}\right|\left|\underline{r}^{\prime \prime}\right|^{2} \alpha^{2} \hat{r}^{\prime \prime} g^{2}$ ives

$$
\begin{aligned}
& \frac{1}{2} \int_{0}^{\infty} a\left|\underline{\underline{r}}^{\prime \prime}\right|\left|\underline{\underline{r}^{\prime \prime}}\right|\left[e^{-t \omega_{1}\left(t-\left|\underline{\underline{r}}^{\prime \prime}\right| / c\right)} \mathrm{n}_{e} \underline{\underline{\kappa}}_{-}-\underline{\underline{k}}_{1}, t-\frac{\left|\underline{\underline{r}}^{\prime \prime}\right|}{c}\right. \text {. } \\
& +e^{+1 \omega_{1}\left(t-\left|\underline{x}^{\prime \prime}\right| / c\right)} n_{e}\left(\underline{k}_{s}+\underline{k}_{1}, t-\frac{\left|\underline{x}^{\prime \prime}\right|}{c} ;\right. \\
& \cdot \int d^{2} \hat{r} " c^{-1 \underline{k}-\underline{s}^{\prime \prime}}\left[r^{\prime \prime} \times\left(r^{\prime \prime} \times \underline{E}_{0}\right) j \cdot\right.
\end{aligned}
$$

The integration over directions may be dore separately. In a spherical coordinate system, $\underline{r}^{\prime \prime}=(\rho, \theta, \phi)$, defined so that $\underline{k}_{s}=\left(\left|\underline{k}_{s}\right|, 0,0\right)$ and $\underline{E}_{0}=\left(\left|\underline{E}_{0}\right|, \eta, 0\right)$

$$
\underline{k}_{s} \cdot \underline{x}^{\prime \prime}=\left|\underline{k}_{s}\right||\underline{\underline{x}}| \cos \theta
$$

and

$$
\hat{r}^{\prime \prime} \cdot \underline{E}_{0}=\left|\underline{E}_{0}\right|(\cos \theta \cos \eta+\sin \theta \sin \eta \cos \phi) .
$$

Using Cartesian components (unit vectors $\hat{e}_{x}, \hat{e}_{y}, \hat{e}_{z}$ ) to describe the vector integrand, (B3) may be expressed,

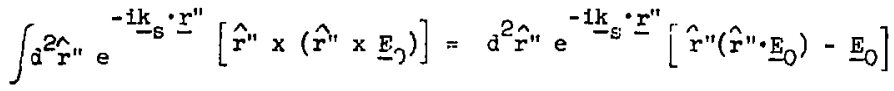

$$
\begin{aligned}
& =\iint \mathrm{d} \theta \mathrm{d} \phi \sin \theta e^{-1\left|\underline{\underline{k}}_{s}\right||\underline{\underline{r}}| \cos \theta}\left[-\underline{\underline{E}}_{0}+\left|\underline{E}_{0}\right| \cos \theta \cos \eta\right. \\
& \cdot\left(\hat{e}_{z} \cos \theta+\hat{e}_{x} \sin \theta \cos \phi+\hat{e}_{y} \sin \theta \sin \phi\right) \\
& \left.+\left|\underline{E}_{0}\right| \sin \theta \sin \cos \phi\left(\hat{e}_{z} \cos \theta+\hat{e}_{x} \sin \theta \cos \phi+\hat{e}_{y} \sin \theta \sin \phi\right)\right]
\end{aligned}
$$

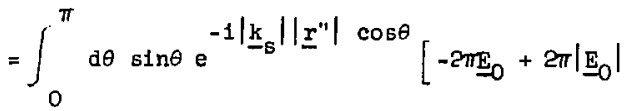


$-192-$

$$
\begin{gathered}
\cdot\left(\hat{e}_{z} \cos ^{2} \theta \cos \eta+\hat{e}_{x} \frac{1}{2} \sin ^{2} \theta \sin \eta\right) . \\
\text { If } x \equiv-\left|\underline{k}_{s}\right|\left|\underline{r}^{\prime \prime}\right| \cos \theta=-\underline{r}^{\prime \prime} \cdot \underline{k}_{s}, \\
\underline{E}_{0 x} \equiv e_{x}\left|\underline{E}_{0}\right| \sin \eta \\
\underline{E}_{0 z} \equiv e_{z}\left|\underline{E}_{0}\right| \cos \eta
\end{gathered}
$$

(B)

then (B4) becomes

$$
\begin{aligned}
& \int_{-\left|\underline{k}_{s}\right| \underline{\mathbf{r}}^{\prime \prime} \mid}^{\left|\underline{k}_{s}\right| \underline{\underline{s}}^{*} \mid} d x \frac{1}{\left|\underline{k}_{s}\right|\left|\underline{\mathbf{r}}^{\prime \prime}\right|} \mathrm{e}^{ \pm x}\left\{-2 \pi \underline{E}_{0}+2 \pi \underline{E}_{0 z} \frac{x^{2}}{\left(\left|\underline{k}_{s}\right|\left|\underline{r}^{\prime \prime}\right|\right)^{2}}\right. \\
& \left.+\operatorname{mix}_{-0 x}\left[1-\frac{x^{2}}{\left(\left|\underline{k}_{-s}\right|\left|\underline{r}^{\prime \prime}\right|\right)^{2}}\right]\right\} \\
& =\int_{-\left|\underline{k}_{s}\right|\left|\underline{r}^{\prime \prime}\right|}^{\left|\underline{k}_{s}\right||\underline{\underline{n}}| \mid} d x \frac{1}{\left|\underline{k}_{s}\right|\left|\underline{r}^{\prime \prime}\right|} e^{1 \times}\left[-2 m \underline{\varepsilon}_{0}+\pi \underline{E}_{0 x}\right. \\
& \left.+\frac{x^{2}}{\left(\left|\underline{k}_{s}\right|\left|\underline{r}^{\prime \prime}\right|\right)^{2}}\left(2 \pi E_{0 z}-m_{0 x}\right)\right] \\
& =\left[\frac{1}{1\left|\underline{k}_{B}\right|\left|\underline{r}^{\prime \prime}\right|} e^{1 X}\left(-2 \pi \underline{E}_{0}+\pi \underline{E}_{0 x}\right)+\frac{1}{\left(\left|\underline{k}_{s}\right|\left|\underline{r}^{\prime \prime}\right|\right)^{3}}\right.
\end{aligned}
$$

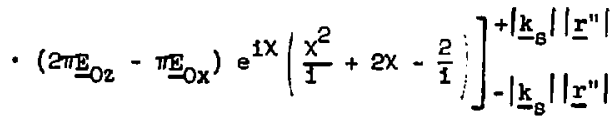

$$
\begin{aligned}
& =\left[\frac{1}{1\left|\underline{k}_{B}\right|\left|\underline{r}^{\prime \prime}\right|} e^{1 X}\left(-2 \pi \underline{E}_{0 x}\right)+\frac{1}{\left(\left|\underline{k}_{8} \| \underline{r}^{\prime \prime}\right|\right)^{3}}\left(2 \pi \underline{E}_{0 z}-m_{-0 x}\right)\right. \\
& \cdot e^{i x}\left[2 x-\frac{2}{1} \mid\right]_{-\left|\underline{k}_{8}\right|\left|\underline{r}^{\prime \prime}\right|}^{+\left|\underline{k}_{8}\right|\left|\underline{\mathbf{r}}^{\prime \prime}\right|} \text {. }
\end{aligned}
$$


This expression is to be integrated over $\left|\underline{r}^{\prime \prime}\right|$. For this one may neglect all but the lowest order term in $l /\left|\underline{I}^{\prime \prime}\right|$. The higher order terms would make little contribution to the Integral and would in any case vanish in a long time limit take later [see note below Eq. (BT)]. Th's leaves, from (B5)

$$
-2 \pi \underline{E}_{0 x} \frac{1}{1 \underline{\underline{k}}_{s}|| \underline{\underline{r}}^{\prime \prime} \mid}\left[e^{i\left|\underline{\underline{k}}_{s}\right|\left|\underline{\underline{r}}^{\prime \prime}\right|}-e^{-1\left|\underline{\underline{k}}_{s}\right|\left|\underline{\underline{r}}^{\prime \prime}\right|}\right]
$$

$\underline{E}_{0 x}$ is simply the component of $\underline{\underline{E}}_{0}$ normal to $\underline{k}_{s^{*}}$. It 1 s convenient to write this in the invariant form

$$
\underline{E}_{0 x}=\underline{E}_{0}^{\perp} \equiv\left(I-\hat{k}_{s} \hat{k}_{s}\right) E_{0} .
$$

Using the result (B6) for the integral over direction \& leaves, for the ent1re expression (B3)

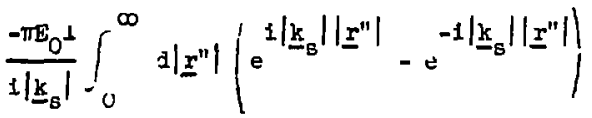

$$
\begin{aligned}
& \text { - }\left[e^{-1\left(\omega_{1}\left(t-\left|\underline{I}^{\prime \prime}\right| / c\right)\right.} \cdot n_{e}\left(\underline{k}_{s}-\underline{k}_{1}, t-\frac{\left|\underline{x}^{\prime \prime}\right|}{c}\right)\right. \\
& \left.+e^{+1 \omega_{1}\left(t-\left|\underline{r}^{\prime \prime}\right| / c\right)} \cdot n_{e}\left(\underline{k}_{s}+\underline{k}_{i}, t-\frac{\left|\underline{x}^{\prime \prime}\right|}{c}\right)\right] \\
& \text { If } \tau \equiv t-\frac{\left|\underline{x}^{\prime \prime}\right|}{\mathrm{c}} \text {. } \\
& \omega_{s} \equiv\left|\underline{k}_{s}\right| c
\end{aligned}
$$

then this becanes 


$$
\begin{aligned}
\frac{-\pi \underline{E}_{0}}{1\left|\underline{k}_{B}\right|} \int_{-\infty}^{t} c d \tau & {\left[e^{1 \omega_{s}(t-\tau)}-e^{-1 \omega \omega_{B}(t-\tau)}\right] } \\
& \cdot\left[e^{-i \omega t} \underline{n}_{e}\left(\underline{k}_{s}-\underline{k}_{1}, \tau\right)+e^{+1 \omega \sigma \tau} n_{e}\left(\underline{k}_{s}+\underline{k}_{1}, \tau\right)\right] .
\end{aligned}
$$

For sufficiently large times, the expression

$$
\int_{-\infty}^{t} d \tau e^{\sin \tau_{n}(\underline{k}, \tau)}
$$

is equivalent to the usual Fourler transform if $n_{e}$ vanishes for lerge $\tau$. Furthermore, the terms in (B5) which were neglected would also vanish in this long time 1 imit, because, for any given $\tau$,

$$
\frac{1}{\left|\underline{r}^{\prime \prime}\right|}=\frac{1}{c(t-\tau)} \rightarrow 0 \text { es } t \rightarrow \infty
$$

Hence sa $t \rightarrow \infty$, (B7) approaches

$$
\begin{aligned}
& \frac{-\pi E_{0}^{1} c}{1\left|\underline{k}_{s}\right|}\left\{e^{1 \omega_{s} t}\left[n_{e}\left(\underline{k}_{s}-\underline{k}_{1},-\omega_{s}-\omega_{1}\right)+n_{e}\left(\underline{k}_{8}+\underline{k}_{1},-\omega_{s}+\omega_{1}\right)\right]\right. \\
& \left.-e^{-1 \omega_{s} t}\left[n_{e}\left(\underline{k}_{\theta}-\underline{k}_{1}, \omega_{\theta}-\omega_{1}\right)+n_{e}\left(\underline{k}_{\theta}+\underline{k}_{1}, \omega_{\theta}+\omega_{1}\right)\right]\right\}
\end{aligned}
$$

Th1s is the desired integration of (BI).

\section{An Integration Needed in Section II.A.3}

A similer, but less compliceted expression

$$
\begin{aligned}
& \int d^{3} D_{1} \int d^{3} D_{2} \frac{1}{\left|\underline{\rho}_{1}\right|} \frac{1}{\left|\underline{\rho}_{2}\right|} e^{1 k_{A} \cdot \underline{\rho}_{1}} e^{-1 \underline{k}_{-B} \cdot \underline{\rho}_{2}} \\
& \cdot g^{(+)^{*}}\left(\underline{r}^{\prime}, t-\left|\underline{\underline{o}}_{1}\right| / c\right)_{B}^{(+)}\left(\underline{r}^{\prime}, t-\left|\underline{\underline{o}}_{2}\right| / c\right)
\end{aligned}
$$


$-195-$

appear i in Eq. (II.14). It is again convenient to use polar coordinates. Replacing

$$
\int d^{3} \rho \text { by } \int d|\underline{\rho}||\underline{\rho}|^{2} \int d^{2} \hat{\rho}
$$

B ives

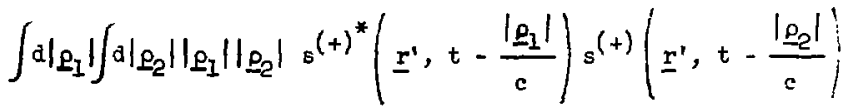

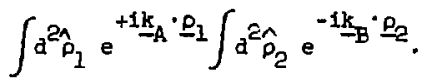

The integration over directions ch n be done in spherical coordinat us

$$
\begin{aligned}
& \int d^{2} \rho_{1} e^{-1 k_{A} \cdot \underline{\rho}_{1}}=\int d \phi \int \sin \theta d \theta e^{1 / \underline{k}_{A}|| \underline{\rho}_{1} \mid \cos \theta} \\
& =\frac{2 \pi}{\left|k_{-A}\right|\left|\underline{g}_{1}\right|} \int_{-\left|\underline{k}_{A}\right| \underline{g}_{1} \mid}^{\left|\underline{k}_{A}\right|\left|\underline{g}_{1}\right|} d x e^{-1 x} \\
& =\frac{2 \pi}{1\left|\underline{k}_{A}\right|\left|\underline{\underline{\rho}}_{1}\right|}\left(e^{1\left|\underline{k}_{A}\right|\left|\underline{\rho}_{1}\right|} e^{-1\left|\underline{k}_{A}\right|\left|\underline{\rho}_{1}\right|}\right)
\end{aligned}
$$

The integration over $\hat{p}_{2}$ gives the same function of $\left|\underline{k}_{B}\right|,\left|\underline{\rho}_{2}\right|$ because this expression is invariant under complex conjugation. This leaves for (Blu)

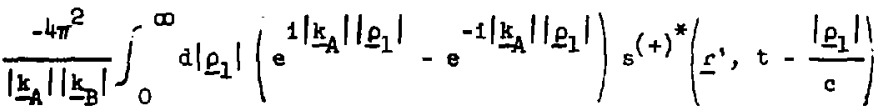

$$
\begin{aligned}
& \cdot \int_{0}^{\infty} d\left|\underline{g}_{2}\right| e^{1\left|\underline{k}_{B}\right|\left|\underline{g}_{2}\right|}-e^{-1\left|\underline{k}_{B}\right|\left|\underline{\rho}_{2}\right|}\left|g^{(+)}\right| \underline{r}^{\prime}, t-\frac{\left|\underline{g}_{2}\right|}{c} \mid=
\end{aligned}
$$




$$
\begin{aligned}
& \left.=\frac{+4 \pi^{2}}{|\underline{\underline{k}}|^{2} \mid} \int_{0}^{\infty} d \underline{\underline{\rho}_{1}} i j e^{1|\underline{\underline{k}}|\left|\underline{\underline{\underline{p}}}_{1}\right|}-\mathrm{e}^{-1|\underline{\underline{k}}|\left|\rho_{1}\right|}\right) \\
& \cdot{ }_{8}^{(+)}\left|\underline{r}^{\prime}, t-\frac{\left|\underline{p}_{1}\right|}{c}\right|^{2}
\end{aligned}
$$

since $\left|\underline{k}_{A}\right|=\left|\underline{k}_{B}\right|=|\underline{k}|$ (see sect. II.A.3).

If $\tau \equiv t-\left|e_{1}\right| / c$, then the $e_{1}$ integration hecomes

$$
\begin{aligned}
& \int_{-\infty}^{t} \operatorname{cd\tau }\left[e^{i|\underline{k}| c(t-\tau)}-e^{-i|\underline{k}| c(t-\tau)}\right] s^{(t)}\left(\underline{r}^{\prime} \tau\right)
\end{aligned}
$$

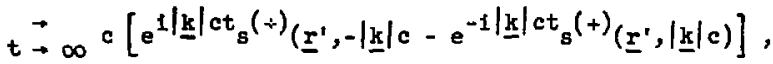

where $s^{(+)}\left(\underline{r}^{\prime},|\underline{k}| c\right)$ 1s the temporal Fourier transform of $s^{(+)}\left(\underline{r}^{\prime}, \tau\right)$. since $(-|\underline{\underline{k}}| c)$ is negative and $\mathrm{s}^{(+)}\left(\underline{r}^{\prime},-\right)$ by definition contalns only positive frequency components, the first terw in the above expression van1shes and the second term describes the 1ntegration in Eq. (B22). Hence, as $t \rightarrow \infty$, (B12) approsches

$$
\frac{4 \pi^{2} c^{2}}{|\underline{k}|^{2}}\left|e^{-1|\underline{\underline{k}}| c t} s^{(+)}\left(\underline{x}^{\prime},|\underline{\underline{k}}| c\right)\right|^{2} .
$$

This is the desired integration of (B9) 


\section{The Design of a Multiple-Beam Spectroscopic Apparatus \\ 1. Previous Conclusions}

In sect. II.A.I, the effect of an elementary two-beam spectrameter was described in falrly simple terms. In this appendix, that first discussion is extended to include anaiyses of several other spectroscopfe gys:ems.

Reviewing briefly, the two-beam system, which is shown in F1g. II-2, was designed to compare light emitted in different directlons from the same volume of plasma. As explatned in II.A.I, the acreen at the end of the system would recelve l1ght from two types of (point) sources:

(a) Sources observed through one beam (A or B, but not both).

(b) Sources within the "common source volume" which are observed through both beams.

An (1sotrcpic) source of the first type would produce on the screen a fairly broad smooth intensity distribution--one whose width would be determined by the diffraction of a single beam. The second type of source would produce a two-beam interference pettern on the screen.

The optical system was designed to use this difference to observe selectively a locelized region within a distributed source. But there is a further complication: All the sources within the canmon source volume might not proüuce colnctdent interference: petterns on the screen. The positions of the intensity maxime would depend upon the precise location of the source.

Some sources, however, would produce ident1cal petterns. As 
explained in Sect. II.A.1, a set of sources which lie in planes which are normal to $\underline{k}_{\Delta} \equiv \underline{k}_{B}-\underline{k}_{A}$ and spaced $2 \pi /\left.\right|_{k_{\Delta}} \mid$ apart (within the cominon source volume) would all produce the same interference pattern on the screen. ( $\underline{k}_{A}$ and $\underline{k}_{B}$ are the wave vectors of the Ifght in beams $A$ and B.) Light from sources located halfway between these "source planes" would produce the opposite or complementary set of fringes on the screen. Hence any overell fringe pattern must represent not the total number of coumon sources, but rather the difference in numbers of two such groups of sources. The system observes not the total. density, but rather the amplitude of one component of the fluctuations in the density of cumon sources.

It should be emphesized that these results do not involve eny interference between light from different sources, as occurs, for example, in a scattering experiment. Fere the light fram the different source points is incoherent and the observed light intensity 1s just the sum of the intensities due to the various point sources-some of which produce sets of interference fringes.

Thus the apparatus must in some way separate a pair of complementary interference patterns which we have called "boams 1 und 2". The quantity of interest is the ilference in intenglty between beams 1 and 2. Th1s difference is proportional to the amplitude of the $k_{\Delta}$ spatial Fourler component of the distribution of light sources within the region observed through both beams $A$ and $B$.

To use these results one actually construct such a device. In planning for this, one 1o isced with soveral further questions: what is a practical way to separate "beams $I$ and $2 " ?$ Are there 
other equivalent but more convenient opt Ical bystems? Is $1 \mathrm{t}$ possible to make better use of the avallable light? And, fingliy, is this observation the only possibility, or is this system one of a larger class of devices with whlch one could make a varlety of optical measurements?

We can note at once that beame $A$ and $B$ need not be restricted to narrow pencils of rays. To obtain more Iight, the apertures which define the beams may be enlerged to parallel slits. This incresses the efflclency, but, as we sholl see, the resulting oystem may be further improved.

\section{A Modifled Two-Beam System}

To separate two complementary interference patterns, one might simply replace the screen with an array of light pipes and direct the light from the locations of the maxima of different pattems into different photomultiplier tubes. But for this to be feasible, the interference fringes would have to be rather videly snaced-which would probably require additional lenses to ragnify the pattern. The resulting system would be falrly complicated. Furthtrmore, such en errangement would only approximate the desired system because interference produces a sinusoldal Intensity diatribution, while a set of ligbt pipes would separate two "square wave" patterns. Indeed, some 11 ght would go Into each phototube, no matter what type of interference occurred.

To see what else ope mlgbt do, consider again our reason for making these Interference patterns. The object is to compere the phase of the light in beam $A$ with that of the light in beam $B$. 
The "Interference petterns 1 and 2 " are simply the result of two posglble phase differences between the light in beams $A$ and $B$. This technique, of course, could be used with sny type of wave, but in an optical system one can also use the fact that light, a trinsverse wave, is characterized by its polarization, as well as by its intensity, f'requency, and phase. This additional property provides an altermate method of making phase measurements, as is expletned in sect. II.B.I, and, more completely, in Ref. 39.

Consider the apparatus shown in Fig. C-1. Here we have agein beams $A$ and $B$. But now we wish to consider this polarszation. A first polarlzer transmits only one component of the light--the same for each beam. The two beams are then linea:ly polarized in orthogonal directions. They are then combined. If the two were In phese, their superposition would agajn be linearly polarized-in the intermediate direction "I" shown in Fig. C-1. If the two componente were $180^{\circ}$ out of phase, their superposition would also be IInearly polarized, but in the orthogonal direction "2". Convenientld enough, these two intermediate polarizations are just beams 1 and 2 , which we wish to separate.

So, to summarlze, the concluaion s: that if the light came from a source lying in one of a set of planes normal to $k_{c}$ and spaced $2 \pi /\left.\right|_{\Delta} \mid$ apart, within the common source volume, when the contributions to beams $A$ and $B$ would be separated in phese by an Integral number of cycles and the light would (all) to into beam 1. Other sources witish the c.s.v. would contribute to beam 2-or both I and 2. And sources outslde the c.s.v. could at most 


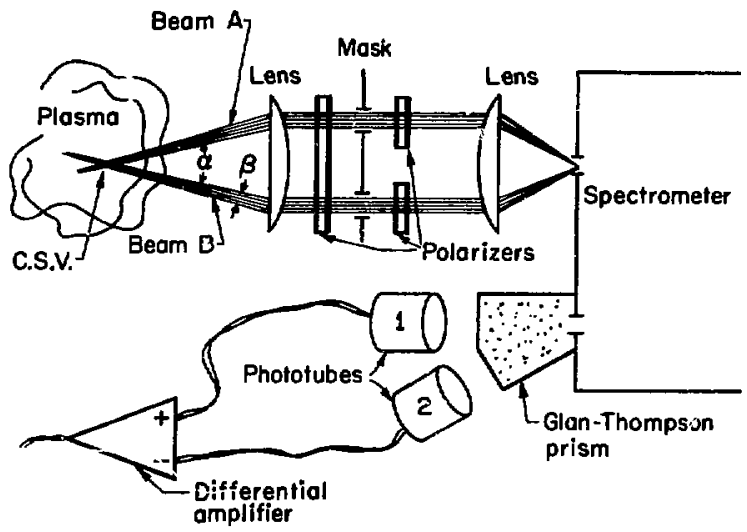

Polorizations

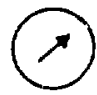

Initial
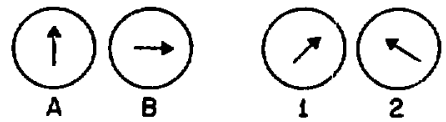

$\times 81.733-2512$

Fig. C-1. A syster: whlch uses polarization to meesure relative phase. 
contribute light only to beam A or only to beam B. This 11ght would be divided equally between beams 1 and 2 and would contribute nothing to their intensity difference.

In the arrangement of $\mathrm{FIB} . \mathrm{C}-1$, the relative phese of beams $A$ and $B$, and benve the resulting polarlzation, also depelds upon the position of the point of observation (where the entrance slit to a spectrometer is Inflcated $j$. Indeed, if there were coherence between $A$ and $B$, then there would still be an interference pattern--and a set of fringes on the screen. But instead of a sinusoidal variation in intensity, there would be i variation of polarization. If a polarizer prlented to select beam 1 were placed before the screen, a set of fringes would appear, and if the polarizer were rotated to select beam 2, the complementary set of fringes would appear. The relative phase of beams $A$ and $B$ is shown not by which intensity pattern appears, but by which pattern corresponds to which polarization, making it possible to, in effect, observe both "patterns" while looking at only one fringe. The presence of spatial variation in the pattern also limits the size of the slit in the screen: Ita widh must be less then the width of one fringe.

Since, in a polarizing system, only a single slit is needed to observe the interference, the seme slit can also serve as the entrance to a spectrometer, as shown in FIg. C-1. In such an arrengement, the frequency resolution would occur after the interferometry, and the lilter shown in Fig. II-2 would not be needed. Thls system, which uses polarization, has several convenient foatures. The separation finto beams 1 and 2 is just what is 
needed, and the posibility of simply mounting the interferometer before the extrance of an existing spectrometer is a big advantage. This not only simplifies construction of the system, it also mini. mizes the number of interferometer-quality optical components needed, because precise equality of path length if not important beyond the entrance to the spectrometer. Light of unwanted phese is blocked by the screen and the following part of the system simply measures the spectre of the accepted 1 and 2 components. As long as these remaln distinct, the signal will be preserved. Unfortunately, the new arrangement has a serlous falling. It makes extremely poor use of the available 1Ight. The system 1s inefficient in two respects. First of all, because the angle $B$ is small, beams $A$ and $B$, As seen from the source, subtend only a small solid angle. Secondly, because less than one fringe of the pattern on the screen is used, most of the light which did go into beams $A$ and $B$ would be lost.

The second l.imitation is clearly removable in principle. One could, for example, construct a system which admitted light through several properly speced slits. But there is a more convenient colution. In the arrangement of FIB. II-2, the interference Iringes on the screen were needed for phase measurements. But the patterns on the screen in Fig. C-I are simply an inconvenience, because the phase measurements aze now made by comparing polariaations.

The small slit at the entrance to the spectrometer may be thought of as a device which combines beams $A$ and $B$. It is 
necessary, as noted above, that this slit be smaller than one fringe of the interference pattern made by beams $A$ and $B$. But this is equivaleni to a requirement that the maximum of a siingle olit diffrection pattern of the slit itself include beams $A$ and B. In other words, within the spectrometer, beams A and B are superimposed. To permit use of a larger slit, another method of combining beams $A$ and $B$ is needed.

\section{The Use of Birefringence}

In constructing an optical system to define and focus beams of polarized light, it is often convenient to use optical elewents made of birefringent materials. We have not yet discussed this possibility, but one can see at once a simple way to produce with such an element a pattern of varying polarization.

The opt1cal system shown in Fig. C-2 includes a calcite rhomb, with the optic axis in the plane of the drawing, as indicated. If a bean of light is incident on the face of the rhomb, its path through the calcite depends upon it.3 polarization. That component of the light which is linearly polerlzed with the electric field vector nomal to the optic axis is prispagated through the calcite as an "ordinery rey." At normel incidence, its direction is unchanged. The other I1nearly polarized component, however, becomes an "extraordinary rey" and a normally incident beam $1 \mathrm{~s}$ deflected by $\sim 6.26^{\circ}$, a change in direction which is reversed at the oppoaite face of the rhomb. The two polarizations thus emerge as soparate beams of light. The varlous rays are again parallel, but one component of the light has been laterally displeced by a 


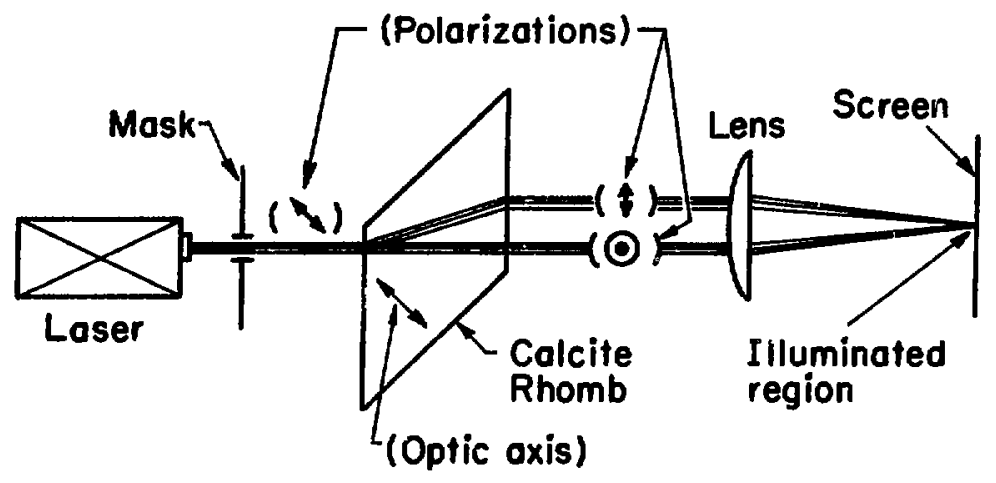

\section{Detail of the illuminated region}

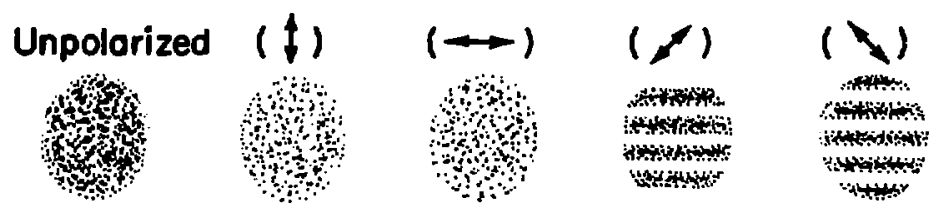

XBL733-2522

F1g. C-2. A demonstration: Interference fringes produced with a calcite rhomb. 
distance approximately 0.11 times the length of the rhomb.

On the arrangement of Fig. C-2, a small gas laser $1 \mathrm{~g}$ used to produce the incident beam, which 1s linearl, polarized in such a direction that it is divided by the calcite into two beams of equal intensity. The light is then focused onto a screen. (More simply, one could place the screen so far away that the divergence of the beams caused them to overlap.) Th = total 1llumination of the focal opot is then rather uniform, but the pattern of polarization conains more detail. If a linear polarizer aligned at $45^{\circ}$ to the polarization of elther beam--that 1a, parallel to the polarization of the original laser beam--is placed before the screen, a set of Interference fringes appears. And if one selects the other, orthogonal, polarization, the complementary set of fringes eppears on the screen.

Th1s result is like that which wes expected from the optical system of Fig. C-1. So, with the calc1te rhomb, one can construct a simple demonstration of the "polarization fringes" which were described earlier. Moreover, this suggests that such an optical component could be useful in the type of spectroscopic system wh1ch ve with to design.

To explore this possibility, we need to consider more systematically our objective. If only two-beam optical arrangements are considered, then thore $3 r 0$ essentlally, four requirements:

1. The epperatus must define s "common source volume"--the intersection of two beams.

2. It must define two directions beams $A$ and $B-$-trom which 
such sources are observed.

3. It must include some spectroscopic element--a filter or a spectrometer--to select a limited portion of the optical spectrum.

4. Finally, the epparatus must measure the correlation, or mutual coberence, of this apectral component of the light in the two beams.

Note that, in any one particular system, the size of the common source volume may be varied inversely with the range of directions included in the two beams. If beams $A$ and $B$ are separetely focused at their intersection (Fig. C-3a), then the c.s.v. Is relatively small, while the range of $\hat{k}_{A}$ and $\hat{k}_{B}$-and hence of $\underline{k}_{\Delta}-1 \mathrm{~s}$ large. This spreed in $k$ may be thought of as due to the small number of "source plabes" In the c.s.v. If, on the other hand, the beams are not focused at their intersection (FHg. C-3b), then the resolution in space is less precise but the resolution in $\mathbf{k}_{A}$. $k_{B}$, and $k_{\Delta}$ is more precige. We shall consider only systems of the type of $\mathrm{FIg}$. C-3a, but one could modify ary arrengement to produce F1g. C-3b. Of course, good resolution in $k_{\Delta}$ also requires good resolution in $\left|\underline{k}_{A}\right|$ and $\left|\underline{k}_{B}\right|$, that is, a sectrameter or a fllter with sufficiently namow pass band.

If the angle between thom is amall, it is correnient to observe beams A and B through difforent sections of a single lens, as shown in Figs. II-2 and C-l. If the remainder of the gystem eccepte only light nearly parallel to the axis, then the observed beane vill intersect in a common source volume around the focal point of the lens. For the interference messurement, the two beams 
$-208-$

(a)

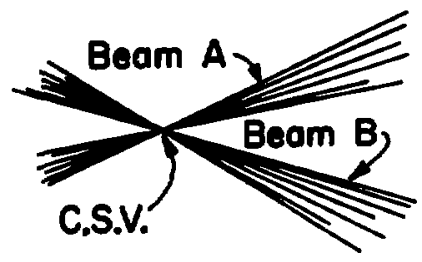

(b)

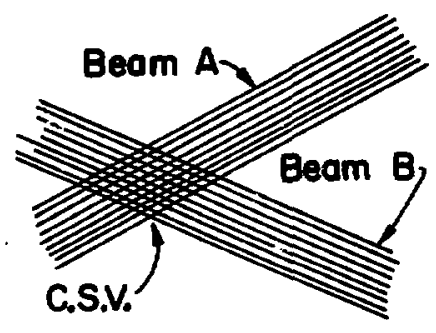

XBL 733-2521

Fis. C-3. Forms of the observation region. (a) Good spatial resolution. (b) Gool wavelength resolution. 
must be recombined beyond the lans. So tive rest of the optical astem must defire and then combine two parallel, adjacent beams or 11ght.

The calcite rhomb in Fig. C-2 alvides one beam of light into two separate parallel beams. If, however, the mask which defines the (single) beas is placed after the calcite element, then two, initially separate, incident beams are deflned and superimposed. Convenieatly enough, this is just what is needed.

The resulting system 18 shown in Flg. C-4. There a lens plus a rhomb not only define and combine beams $A$ and $B$, but also determine their polarizations--something which required separate polar1zers in the setup which we firat alacussed (F1g. C-I). The first polarizer, which insures that we start with a single component of the light is, however, otill mquired.

This gain in simplicity is not, however, the principal difference between the two designs (F-gs. C-1 and C-4). Nore important is the change in the distribution of light over the 1lluminated portion of the screen (before the spectrometer). In the new armangement, the two-boam interference fringes are absent. Beams $A$ and $B$ are parallel at the screen and their phase difference is constant within the limitations of the collimetion of each single beam.

An Isotropic point source within the c.s.v. will here produce on the screen a single olit diffrection pettern due to either bam alone. (since this $1 \mathrm{~s}$ ahead of the opectrometer, consider also that the source 1s monochromatic.) We can distinguish these 


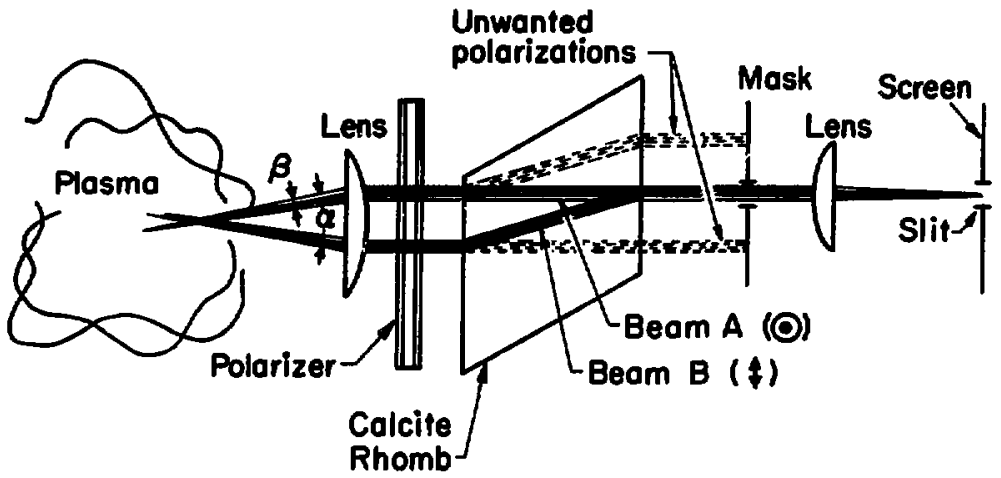

XBL733-2520

F1g. C-4. A two-beem spectroscople system in which a calcite rhomb 18 used to comblne the beams. The slit in the screen at the right 1s the entrance to a spectrometer. Beams 1 and 2 are seperated at the spectrameter ex1t. 
with a polarizer but, alnce the two components cose from the oame slit, the two patterne will be ldentical, provided that the two distributions of phese ecross the first slit are 1dentical. (We assume, of course, that any polarization dependence of the effect of the slis is negligible.) If the two afferaction patterns are 1dentical, than the varlations in phase across the screen will also be the same for each component. Therefore the phase difference between the two-mand hence the polartzation of their superposition--will be constant across the screen. The entrance sllt to the spectrometer may be made large enough to admit a substantiel part of the 11ght. The result is a more efficient system.

However, the second slit should probably st1ll be narrower than the central maximum of a single-slit diffraction pattern of the first slit. ${ }^{56}$ This would insure that the phases of the $A$ and $B$ components could not vary much across the second slit. Then their relative phase, and hence the polarization of thelr superposition would also be approximately unflorm, and each point source within the plasma would contribute with a single polarization to the output light.

This is not to eay that a larger second slit could not posk1bly be used. It could, but that would ellow the polarizetion of the light from some polnt sources to vary across the width of the 8lit. The overall effect, given by an average over the slit area, could then include some cancellation between different contributions. Now the 1llumination of the second slit is just a sum or contributione (Huygens components) from the light which goes 
through different portions of the first slit. If a source were near the edge of (or, for that matter, outside of) the common source volume, then the distributions in phase across the flrst slit, and hence the distributions in intensity and phase across the screen would be differert for the light observed through the bears $A$ and $B, 57$ and the polarizaton of the total light would vary with position. If the second slit were wider then the suggested limit, there could be some cancellation of effect. But this would r.ut happen with all sources. A source near the center of the c.s.v., for example would produce unfform distributions of A and $B$ across the first sl1t, making two identical patterns on the screen. The polarization of light from this source would not change, even over distances greater than the suggested slit width.

Thus, if a larger second slit were used, our simple statement that each common source contributes with one polarization to the output light would not be strictly valid. Some sources would, but the effect of others would be reduced or lost in averaging across the slit. Only some restricted portion of the c.s.v. would still make a definite contribution to the output signal. Other common sources would contribute only background light, as do the sources sien through just one beam.

Finelly, the width of the second slit also limits the spatial realution, since the C.s.v. Is just an image of the second slit. Enlarging the slit would enlarge the c.s.v. but, on the other hand, reducing the slit to lese than the limiting width would not further Improv the resolution, since the image would then be 
diffraction limited.

Even with thls limitat.on on the second slit, the system shown in Fig. C-4 vould st111 eccept most of the light emitted in directions $k_{A}$ and $k_{B}$ by common sources. It thus overcomes the second of the two limitations--noted at the end of sect. 2.3--of the apparatus shown In FIg. C-1. If we wish to study only light emitted into two narrow beams, the apparatus of F1g. C-4 satisfies our requi rements.

\section{Mult1ple-Beam Systems}

The more efficlent two-beam spectrometer which we have now designed has both the advantages and the limitations of the oriBlal concept. Amy device which accepts only two narrow beams can use only a small part of the avallable 11ght. To further improve the design we must conslder a more general class of systems. On doing so, we can constder observation of various other aspects of the source distribution as well.

A stmple two-beam epparatus responds, as we have seen, to a nerrow portion of the spectrum of the spatial distribution of sources within a localized "comnon source volume." One has at once two types of spatial resolution: A coarse definition of an

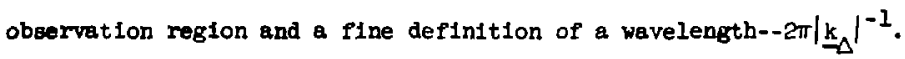
ane can make a local measurement, but only of fluctuations. Such an optical syotem is hardly the only possibility: We have yet to explore the range of posstble observations. Could one, for example, oelect all the light emitted from within some localized volume and still reject that emitted from other reglons? 
Before considering other arrangements, it is conventent to aumarize analytically the jroperties of the system already designed (Fig. C-5). This consists of a source region, a polarizer, a ftrst opticai system (I), a slit, and a second optical system (II). System II, beyond the entrance slit to the spectrometer, selects a narrow portion of the optical spectrum, separates two polerizetion components--1 and 2--and measures the difference betw on thase two total intensities.

The preceding portion of the apparetus, System I, defines two beams, $A$ and $B$, distingulshed by their polarizations. A point source within the source region thus produces two flluminations (Including zero emplitude as a possibility) of the entrance slit to the spectrometer. These rary in amplitude and phase along the slit. The effect of System I may thus be represented by two complex transfer functions, $\phi_{A}$ and $\phi_{B}$ (see sect. II.B.2). If a point source of ligsht of frequency $\omega$ is then represented by a (complex) source strength $p(\omega ; t)$, that is, by a source density

$$
s\left(\underline{r}^{\prime}, \omega ; t\right)=p(\omega ; t) \delta\left(\underline{r}^{\prime}-\underline{r}_{0}\right)
$$

toen the resulting disturbances at the slit are determined by the products $\left(\phi_{A} p\right)$ and $\left(\phi_{B} p\right)$.

$\phi_{A}$ and $\phi_{B}$ depend upon the locetion of the source $\left(\underline{y}_{0}\right)$ and the location along the slit of the point of obse, tion $\left(x^{\prime \prime}\right)$. For completeness we include mention of a position across the width of the slit ( $\left.y^{\prime \prime}\right)$. The intensities of light in beams $A$ and B which passes the slit are 


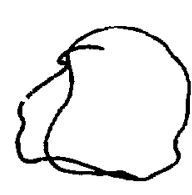

Source

region

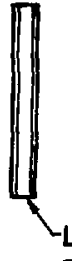

Linear polarizer

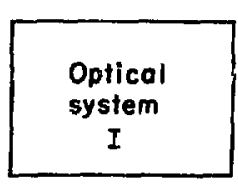

Spectrometer entrance slit

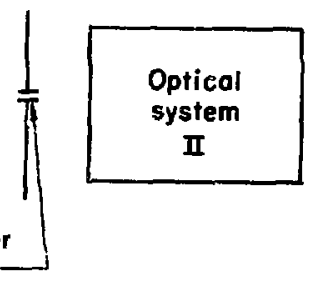

$x$ X $733-2519$

F1g. C-5a. Functional elements of the optical system. 


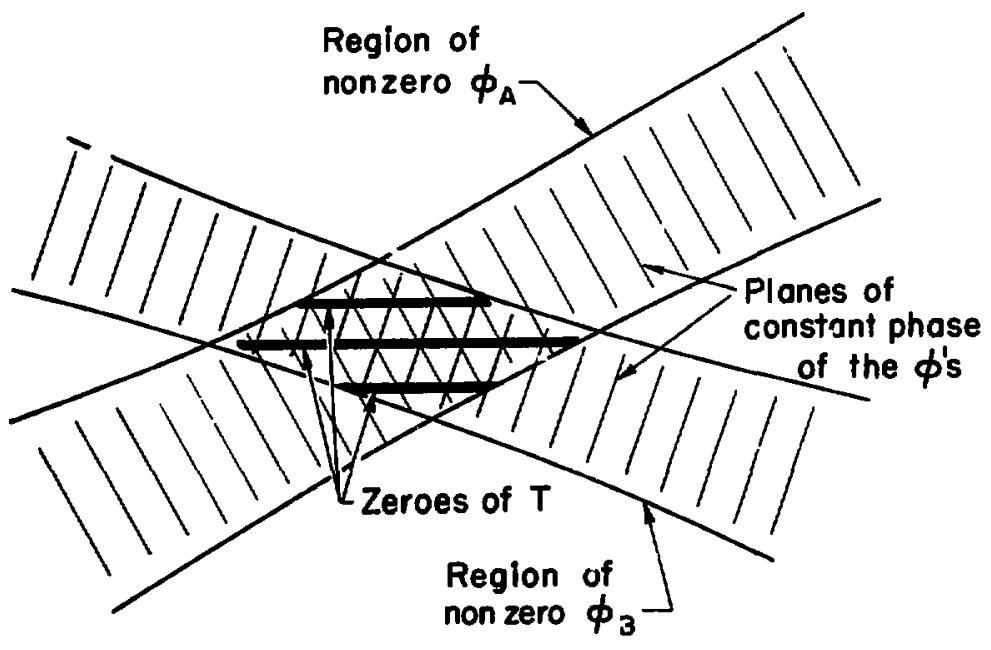

XBL733-2513

F1g. C-5b. The two transfer functions and the observed component of the source distribution for a simple two-beam system. $\eta$ is everywhere zero tscept in the intersection of the two beans. 


$$
I_{A, B}=\int_{x_{1}^{n}}^{x_{2}^{\prime \prime}} d x^{\prime \prime} \int_{y_{1}^{\prime \prime}}^{y_{2}^{\prime \prime}} d r^{\prime \prime}\left|\phi_{A, B}\left(x^{\prime \prime}, y^{\prime \prime}, \underline{x}_{0}, \omega\right) p(\omega ; t)\right|^{2}
$$

due to the point source. $\left(x_{2}^{\prime \prime}-x_{j}^{\prime \prime}\right)$ and $\left(y_{2}^{\prime \prime}-y_{1}^{\prime \prime}\right)$ are the dimensions of the slit.

A distributed source is described by a source density, $s\left(\underline{r}^{t}, \omega ; t\right)$. We assume that the source is incoherent. That is, the total intensities are the sums of the Intensities due to the separate elements of the source:

$$
I_{A, B}=\frac{1}{\pi} j d^{3} r^{\prime} \int_{x_{1}^{\prime \prime}}^{x_{2}^{\prime \prime}} d x^{\prime \prime} \int_{y_{1}^{\prime \prime}}^{y_{2}^{\prime \prime}} d y^{\prime \prime}\left|\phi_{A, B}\left(x^{\prime \prime}, y^{\prime \prime}, \underline{r}^{\prime}, \omega\right) s\left(\underline{r}^{\prime}, \omega ; t\right)\right|^{2}
$$

Optical system II, however separates not these intensities, but those of the two intermediate polarization--1 and 2. The resulting signal, the difference in intensity, is

$$
\begin{aligned}
& Y(\omega ; t)= I_{2}(\omega ; t)-I_{1}(\omega ; t) \\
&=\frac{1}{\pi} \int d^{3} r^{\prime} \int_{x_{1}^{\prime \prime}}^{x_{2}^{\prime \prime}} d x^{\prime \prime} \int_{y_{1}^{\prime \prime}}^{y_{2}^{\prime \prime}} d y^{\prime \prime}\left[\left|\frac{1}{\sqrt{2}}\left(\phi_{B} s+\phi_{A} s\right)\right|^{2}\right. \\
&\left.-\left|\frac{1}{\sqrt{2}}\left(\phi_{B} s-\phi_{A} s\right)\right|^{2}\right] . \\
&=\frac{1}{\pi} \int d^{3} r^{\prime} s^{*} s \int_{x_{1}^{\prime \prime}}^{x_{2}^{\prime \prime}} \int_{y_{I}^{\prime \prime}}^{y_{2}^{\prime \prime}} d y^{\prime \prime} \\
& \cdot \frac{1}{2}\left[\left(\phi_{B}^{*}+\phi_{A}^{*}\right)\left(\phi_{P}+\phi_{A}\right)-\left(\phi_{B}^{*}-\phi_{A}^{*}\right)\left(\phi_{B}-\phi_{A}\right)\right] .
\end{aligned}
$$


$-218-$

Defining an emitted spectrum as wo did in Sect. II.A.3,

$$
\mathscr{L}\left(\omega ; \underline{r}^{\prime}, t\right) \equiv s^{*}\left(\underline{r}^{\prime}, \omega ; t\right) s\left(\underline{r}^{\prime}, \omega ; t\right)
$$

and reducing the above result we have

$$
Y(\omega ; t)=\frac{1}{\pi} \int a^{3} r^{\prime} d_{x_{1}^{\prime \prime}}^{x^{\prime \prime}} d x " \int_{y_{1}^{\prime \prime}}^{y^{\prime \prime} 2} d y "\left(\phi_{B}^{*} \phi_{A}+\phi_{A}^{*} \phi_{B}\right) .
$$

The last factor can be written,

$$
\begin{aligned}
\phi_{B}{ }^{*} \phi_{A}+\phi_{A}^{*} \phi_{B} & =2 \operatorname{Re} \phi_{A}^{*} \phi_{B} \\
& =2\left|\phi_{A}\right|\left|\phi_{B}\right| \cos \theta
\end{aligned}
$$

where $\theta$ is the difference in phase between the complex valued quant ties $\phi_{\mathrm{A}}$ and $\phi_{\mathrm{B}^{*}}$. Defining

$$
\begin{gathered}
T\left(\underline{r}^{\prime}, \omega\right) \equiv \frac{2}{\pi} \int_{x_{1}^{\prime \prime}}^{x_{2}^{\prime \prime}} d x^{\prime \prime} \int_{y_{1}^{\prime \prime}}^{y_{2}^{\prime \prime}} d y^{\prime \prime}\left|\phi_{A}\left(x^{\prime \prime}, y^{\prime \prime}, \underline{r}^{\prime}, \omega\right)\right| \mid \phi_{B}\left(x^{\prime \prime}, \underline{x}^{\prime \prime}, \underline{r}^{\prime}, \omega \mid\right. \\
x \cos \theta\left(x^{\prime \prime}, y^{\prime \prime}, \underline{r}^{\prime}, \omega\right)
\end{gathered}
$$

we have

$$
Y(\omega ; t)=\int d^{3} r^{\prime} T\left(\underline{r}^{\prime}, \omega\right) \&\left(\omega ; \underline{r}^{\prime}, t\right) .
$$

In distinction to the $\phi^{\prime} \mathrm{s}$, $T$ is a real (but not necessarily positive) quantity.

We have here a formal representation of the two types of opt 1 cal interference which occur in the system: Diffraction due to the superposition of various flugens components of each beam defines the beams and determines each of the two transfer functions $\phi$. 
Interference between the two beams is represented by the Interference between the two functions $\phi$. This determines the observed component of the source distribution--described by $T\left(\underline{r}^{\prime}, \omega\right)$.

For our two-bean apparatus, the general form of these three functions is shown in Fig. 5B. $\left|\phi_{A}\right|$ and $\left|\phi_{B}\right|$ are nonzero only within the respective beams. Therefore $T$ is nonzero only within their intersection. Thls defines the c.s.v. The relative phase of the two (the $\cos \theta$ factor in T) varies within the c.s.v. as shown, defining a source wavelength, or $k$.

In this arrangement, the small size of the solld angle through which the system accepts light is due to each of the $\phi^{\prime}$ s separately, while the object of the messurement is defined by $T$. To use more of the avallable light, we need other "beams" $\phi_{A}$ and $\phi_{B}$, ones which interfere to define elther a $T\left(\underline{r}^{\prime} ; \omega\right)$ like that we have already, or else some other $T$ of particular interest.

If, in the gystem of Fif. C-4, one specifies an opt Ical wavelength, and an arrangement of lenses and calcite, then both the location of the c.s.v. and the wavelengtb $2 \pi|\mathrm{k}|^{-1}$ are determined (the c.B.v. by the Imaje of the entrance olit to the spectrometer, and $\left|\underline{k}_{\Delta}\right|$ by the angle $\alpha$ which 18 flxed by the distance by which the calcite element diaplaces an extreordinary ray). Firally, the arrection $\hat{k}_{\Delta}$ is deterwined by the directions of the beams, that $1 \mathrm{~s}$, by the location of the aperture in the mak behind the calcite. Hor, if $\alpha$ is and, the ranize of posstble positions of this beemlefining aporture corresponds to only a wenge of $\hat{k}_{\Delta}$. And the locations of the maxim of $T$ (1.e., the "phase of $T$ ") are also 
unchanged because the exact center of the c.s.v. Is always a (zero order) maximum. So, over some range at least, $T\left(\underline{r}^{\prime}, \omega\right)$ is independent of the exact location of the eperture which defines the beams (at least near the center of the c.s.v.). The resultIng existence of many equivelent sets of beams A and B suggesto the possibility of obtaining wore light by using eeveral pairs of beams at once--In a multibeam system.

So far ve heve insured a locelized meagurement by defining separate interfering beans $A$ and $B$ with a woll localized intersection. We shell for now rotain this approach--which requires some sort of mak to define the separate beams.

To use a Iarge solid angle while defining ceparete heams, one Wght eaploy a wak with way slits. Such an assembly is shown in Fix. C-6. The slit opecing (In the nask) has been set at tulce the distance of the displacenent due to birefrigence so that the beans ere distinct. $\phi_{A}$ and $\phi_{B}$ now describe two sets of "ceams $A$ " and "boame $\mathrm{B}^{\text {". }}$

Bans 1 and 2 ere eguin the laternediate polerizetiong--8eparated et the output of the spetronter. So, agein, only cources rieved through both $A$ and $B$ end contribute to the signal. And elnee the two only interaet near the focus of the flrst lens, a locelised c.s.v. Is egeln defind.

Within th c.s.v., howver, th alturtion is different. The calculation of eitber $\phi_{A}$ er $\phi_{B}$ in the foes plane of the first Lens reduces to the colution of a siple Frsubhofer diffraction problen for $n$ identieal, evenly apced slits. Fer the center of 


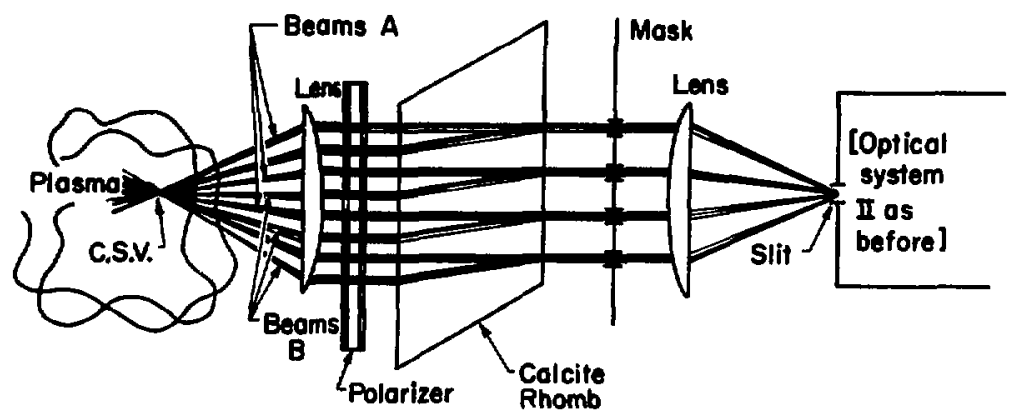

XBL753-2517

F1g. C-6a. A multiple-beam system. 
$-222-$
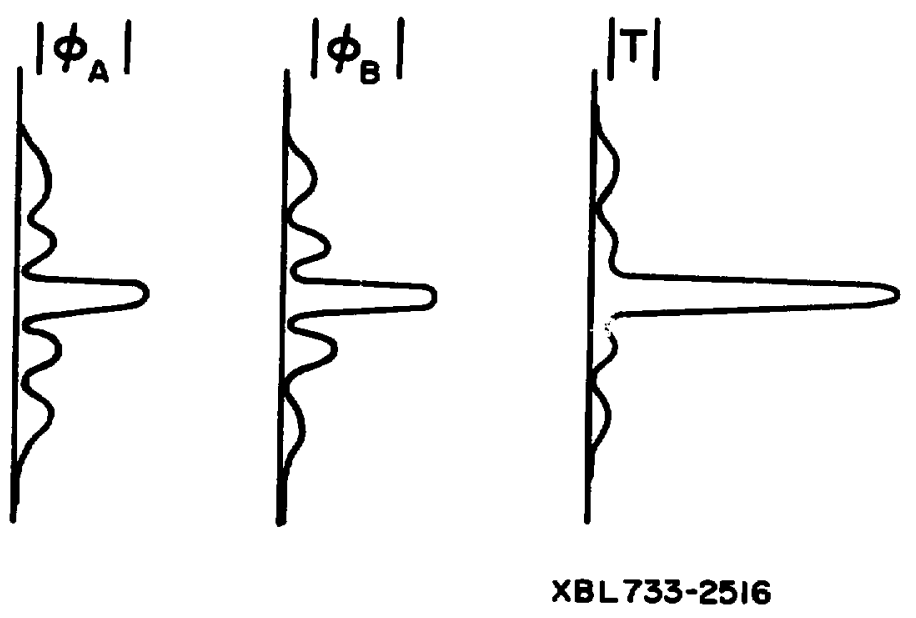

Fig. C-6b. The effect of sources in the focal plane of the first lens. A cross section of the central portion of the c.s.v. 
the c.s.v. the various functions have the form shown in Fig. C-6b. The system thus achleves, at least in this region, a measurement of all the light from a small volume. This is comething which colld not be done with two heams because the angle $\alpha$ between the beams 1s, of course, larger than the angle $\beta$ subtended by either. If $\beta$ now denotes the range of directions included in either set of beams, then in Fig. C-6a, $\beta$ 1s larger than $\alpha$ and the system permits a different kind of observation.

The situation is more complicated, however, because the effect of sources near the edge of the c.s.v.--particularly at other maxime of $\phi_{A}$ and $\emptyset_{B}$--and the effect of sources before or behind the focel plane of the first lens remain to be considered. In any cese, this arrangement is clearly not just a more efficient version of a two-beem spectrometer. The difference is due to the interference between the various "beams $A$ " or "beams $B$ ". These interfere with each other as well as with the other polarized components to produce beams 1 and 2 .

It $1 \mathrm{~s}$ of interest to note that the nature of this 1nterference depends upon the width of the entrance flit to the spectroweter. The narrow slit implicitly asumed above admits less than one fringe of the interference pattern. A wider slit wuld have a different effect. This is consigtent with our earlier pleture of the olit as an element which combines by diffraction light from different directions. A very narrow slit combines all Incident 11ght; a wider blit only combines nearly parallel beams. Clearly, th1. system should be discussed wore completely and carefully-- 
probably with the help of numerical analysis. Such a discussion we defer for now to consider instead a different design.

In the device of Fig. $c-6$, the entrance slit to the spectroneter defines a c.s.v. by refecting umanted light. But if any light is refected by a mask after the many beams are combined, then interference among them cannot be ignored. To obtain more light while making an observation such as one would obtain with a twobeam system, it is necessary to independently define the component beams. A system which does this is shown in Fig. C-7.

Here we have used the fact that in preceding systems the gecond lens is not really needed to define a c.s.v. The light from such sources is already focused--at infinity. One can define a c.s.v. and insure coherence by placing a defining aperture sufficleatly far avay. This can be done separately for each component pair of beams A and B. One must simply add a set of collimating slits to the apparatus. Beyond the collimator, a single lens may be used to focus the light onto the entrance slit to the spectram-ter. Th1s slit should now be large enough to admit all of the 116ht transwitted by the collimator. 58

The polarization components 1 and 2 , and hence their fatensity difference are almply the sum of contributions from various component beams. There 18 no interference here at all and the system Is simply the sum of many two-beam assemblies--each with the same $T\left(\underline{r}^{\prime}, \omega\right)$. 


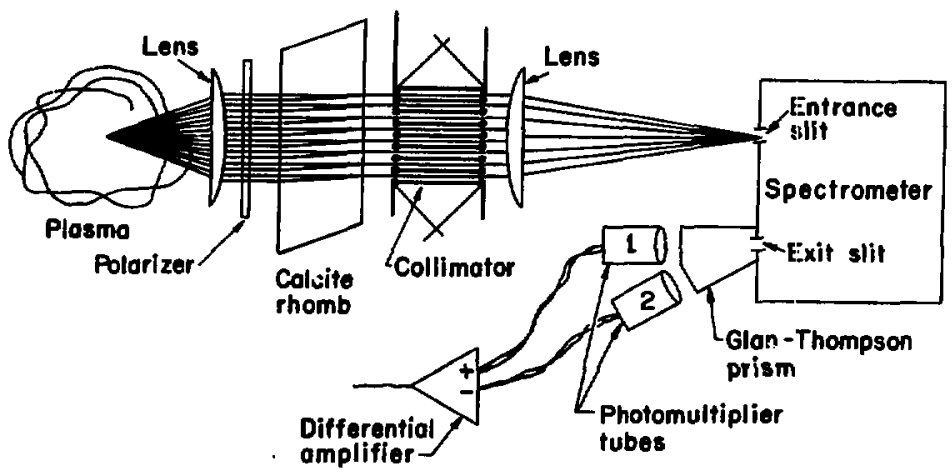

XEL7210-4329

F18. C-7. A system with several independently collimated pairs of beans. 
5. Spectral Width, Beam Divergence, and the Quality of the

Opticsl Components

Several tdealizations bave been used in this analysis. Are the conclusions realistic? To answer this, one must consider depertures from the model system.

A calclte rhomb is to be used to combine two beams of nearly monochromatic light. Within the calcite, however, the two beams differ not only in peth, but elso in propegation velocity. This afference in velocity leads to a difference in optical path length--a difference which could, if necessary, be reduced with an additional birefringent optical element. 59

Differences in path length are of great importance in any interferometric optical apparatus. If the difference in path exceeds the coherence length of the l_ght, two beams with an initial partial coherence w1ll almost sertainly become incoherent and no net interference w111 be seen.

The ordinary ray is unchanged in direction within the calcite. Ite velocity is just $c / n_{0}$, where $n_{0}$ is the ordinary index of refrection, 1.658. Therefore the optical path 1s simply $s_{0}=1.658 \mathrm{D}$, where $D$ is the length of the calcite element.

The extroordinary ray is churacterized by two velocities, a phese velocity and a group velocsty, which differ both in magnitude and in direction. Conveniently, however, the projection of the group velocity onto the direction of the phase velocity 18 equal to the phase velocity. For normel lacidence, this directtion is the seme as that of the ordins yy ray--normal to the surface. 
And because the light is focused at Infinity and normal to the faces of the rhomb before and after, the optical path length within the calcite (the free apace wavelength times the number of wavelengths along a ray) 1s found from just this normal component of the propagation. The magnitude of this phese velocity (which involves both $n_{0}$ and $n_{x}$, the extraordinary index of refraction) is approximately $\mathrm{c} / 1.549$, so the optical peth length $s_{x}=1.5490$. The optical path length difference introduced by the birefringent element 18

$$
s_{0}-s_{x}=0.1090
$$

So, if the length of the calcite is one centineter, the coherence length of the light must be considerably more than one millimeter, or corrections must be mede to avold loss of coherence. In practial terms, this weans that the width of the spectral feature should be less than about two angstroms.

In our analysis of the effect of the calcite, we have considered only rays at normal incidence and used the conventeritly simple result: One component of the light is laterally diapleced by $0.1097 \mathrm{D}$. The effect of tirefringence upon Ileht Incident other than normally 18, of course, more complicated. Two orthogonal polarization components of tha beal are then both displaced by the calcite and their resulting separation varies with the direc. tion of incidence. Arcill that, at norwal Incidence, the $x$-pol.arization 1 soflected by $6.26^{\circ}$. Cleerly, our analysis 18 adequate only so long as eny depertures from normel incidence are wuch lese 
than this. We can hence consider only light from scurces sufficlently close to the focel point of the first lens.

In optical interferometry, much care is often required to eliminate mechanical vibrations of the components of the system. But 1. a system designed to observe high-frequency phenomena, one can certainly 1gnore low-frequency virations. Meshanical vibratlons are in general of much lower frequency than oscillations in a plasma and should therefore present no problems.

Even if one wishes to otserve low-frequency phenomena, one needs to consider only relative changes in the length of the paths of the two components in the interference. Through most of our system the two paths are identical or at least adfacent and much mechanical vibration may st1ll be ignored--a result wit.1ch further 1llustrates the convenfence of using polarization to ciefine the interfering beams.

Finally, one must consider what optical quality is needed in the varfous components of the system. If the apertures were pinholes, such requirements would be minimal, but sl:ts have keen used to obtain more light and one must insure that the nature of the 1nterference does not vary acrose the entrance to the spectrometer or across the apertures which define the beams.

clearly, any imperfections in the system will distort the Image of the plasme and reduce the accuracy of the measurement. That 18, to a given point at the entrance to the spectroneter, there corresponds some observed source distribution $\left[T\left(x^{\prime \prime}, y^{\prime \prime}, \underline{r}^{\prime}, \omega\right)\right.$ with $x^{\prime \prime}, y^{\prime \prime}$ fixed]. If the opticel quality is poor, this will 
differ from the desired distrilution.

More serious than this, however, is the passibility that optical imperfections may destroy the interference altcgetler. Th1s will occur if the various points across the entranse to the spectrometer obscrve dfferent source distrfbutions. That is, a given point source within the comwon source volume 1lluminates through each of the beams $A$ and $B$ a fintte length of the entrance to the spectrameter. The two 1llumination patterns $\left.\phi_{\mathrm{A}}, \mathrm{x}^{\prime \prime}, \mathrm{y}^{\prime \prime}, \underline{\mathrm{r}}^{\prime}, \omega\right)$ with $\underline{\mathbf{r}}^{\prime}$ fixed must vary in the same manner along the length of the sl1t, or the polarization of their superposit: $n$ will vary with $x^{\prime \prime}$. That is, the function $T\left(x^{\prime \prime}, \mathrm{y}^{\prime \prime}, \underline{r}^{\prime}, \omega\right)=\frac{2}{\tau}, \phi_{\mathrm{A}}|| \phi_{\mathrm{B}} \mid \cos \theta$ may vary w1th $x$ " because of the (same) change " $r_{1}\left|\phi_{A}\right|$ and $\left|\phi_{B}\right|$, but must not vary in sign because of the charges Ir relative phase, $\theta$. To prevent such a loss of interference, the optical elements before the spectrometer (opt1cal system I) wust be of good quality. The two interfering beams go through different portions of the Lenses and the calcite and any lens akerrations or curvature in the faces of the calcite will lead to a difference between $\phi_{A}$ and $\phi_{B}$, the two interfering illuminations.

This sort of difficulty would also result from a wedge in the calc1te rhomb. If the two faces are flat but not parallel, the beam cannot be nomally incident at both faces. There will be a refraction at one or both surfaces. This is not in itself destructive of the interference. But because the indices of rePraction are unequal, the two beems $k \pm l l$ be refracted alfferently. The resulting difference in direction can destroy the interference. 
The refraction is described by snell's law, which involves the phase velocities. These are approximately normal to both faces of the rhomb (Inside the calcite as well as outside). If there Is a cmall wedge angle $\phi$ to the rhomb, each veam will be defected by an angle $\theta=(n-1) \phi$. This will cause a difference in direction

$$
\begin{aligned}
\Delta \theta & =\left(n_{A}-1\right) \phi-\left(n_{B}-1\right) \phi=\left(n_{A}-n_{F}\right) \phi \\
& =(1.658-1.549) \phi \cong \frac{1}{9} \phi
\end{aligned}
$$

Therefore, the exposed portions of the faces of the rhomb must be parallel to within a few optical wavelengths. But they need not be parallel to within a fraction of one wavelength--because only differences in deflection are important.

Simllarly, a small amount of wedge in the first polarizer (that before tha rhomb) would not be detrimental, so long as the dilserent beams were deflected equally.

The optical elements beyond the entrance slit to the spectrometer (optical system II) ray be of lesser quality. All that Is required is a measure of the intensities of polarization components 1 and 2 of one portion of the optical spectrum. The optical elements--such as the diffraction grating--must keep these two components distinct, but precise equality fil path length is no longer important. It is for this reason that the interference is done kefore the spectrometer.

Finally, all of the optical elements wust be of sufficlent quality to avold loss of light through partial reflection, absorp- 
t1on, or scattering. Any decrease in light intensity will, of course, degrade the final algnal. 


\section{Studies of the Plasma with Lazgmuit Probes and with Conventional Spectroscopy}

In preparation for the multiple-beam spectroscopic observations, the plasma produced in the beam-plasma device was observed with some standard diagnostic apperatus. The besic pleswe peramters were already known from other work done with this plasma. 51 (for a description of the beam-plesme device, see sect. IV.a and Ref. 51.) The plasma electron density had been found to be a few times $10^{13} \mathrm{~cm}^{-3}$ and the electron temperature bad been estimated Prom spectroscopic observations to be greater than $4 \mathrm{eV}$, perhaps 8s high as $20 \mathrm{eV}$. The fon temperature was. less than a few tenths of an ev.

To check the temperature measurement and to explore the possibility of using a probe to launch waves in the plasma, several Iangmulr probes were made and used to obtain standard probe characterlatics (current vs blas voltage). These curves had the expected form, showing an ion saturation region, where the trace was linear (with a non-zero slipe) and an expontential increase in current as the voltage became less negative. The Indicatar alectron temperature was about $4 \mathrm{eV}$, somewhat lower than had been found In the preceding work. ${ }^{51}$ This is not surprising, since the earlier spectroscopic data showed mainly the central region, within the electrcn beam, whlle our probe data were taken outside of the beam, where the electron tempera, ire would certalnily be lower. (Probes could not be used within the beam, because a probe there would quickly have vaporlzed.) 
In a heliuw plasm with $\mathrm{T}_{e}=4 \mathrm{eV}$, Ion vaves should heve a velocity of $10^{6} \mathrm{~cm} / \mathrm{sec}$. This 18 only an estimate, however, since the plane was strongly nonunifors, the electron temperature vas bigber in the center, and the erfect of neutral atoms (and of doubly cbarged lons) may not be negligible. To observe fon waves directly, and to show that a probe could be used to launch such waves, a mir of Langmuir probes were inserted in the plaswe. The probes vere both blased to draw fon current and were placed a few centimeters apart, with one directly downetrean (1,e., on the same magnetic field lines) from the other. The upstream probe was used as a transmitter and the downatream probe was used as a rocelver. A serles of one microsecond pulses (or a few voiis, positiva) were imposed on the trensmitter, and the resulting fluctustions in recelver current were andlyzed with a boxcar integrator. Figure D-1 show the results obtalned at three recelver positions. Each trace shows recelver curreni 38 a function of time--a 10 Hsec sweep frow left to right. several propagating modes can be seen. There Is a fast wave (the Inftial negative pulse) with a velocity exceedIng $10^{7} \mathrm{~cm} / \mathrm{sec}$, whict. 1s prokably a potential fluctuation. There 10 a slow wave, only viaible in the firat trace (but seen at greater distances at later times in other traces not shown here) with a velocity of a few times $10^{5} \mathrm{~cm} / \mathrm{sec}$, which probably 1 nvolves the noutrel gas. Finally, there is a pulse with interuediate velocity which 18 seen in all three traces. This disturbance moves with a velocity $\sim 1.3 \times 10^{6} \mathrm{~cm} / \mathrm{sec}$, acceptatly close to the expected Ion sound speed. (The velocity appears to increase slightly with probe 

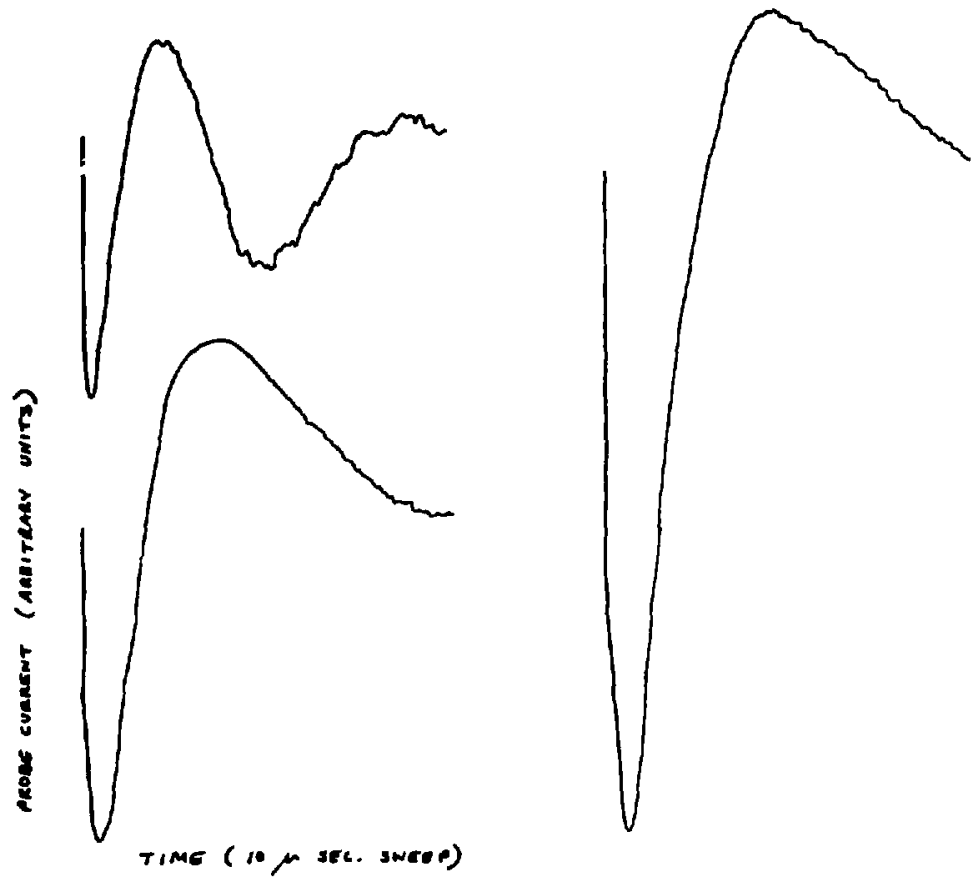

XBL $735-670$

Pig. D-1. Langmulr probe observations of pulse propagation in the plasma. Top left: probe apacing 1-1/4 1n.; bottom left: probe spacing $2-1 / 161 \mathrm{n}$; right: probe spacing 2-7/8 1n. (These are tracings of $X-Y$ recorder plots of the output of the boxcar integrator.) 
spacing. This is not surprlaing. The probes visibly perturbed the plasm and the perturbation was smaller when tbey were farther apart.) From these results 1t was concluded that our estimate of the Ion sound speed in this plesma was correct, that plasma density disturbances would propegate at thls spead for seversl centimeters along the mognet1c field (without, in particular, being damped by collisions with the neutral gas) and that a negatively biased Lanomuir probe could be used to launch such waves.

In order to select the spectral feature best sutted for mult1ple-beam observations, a complete emission spectrum of the plesma was recorded, using a monochromator with eutcmatic scan connected to a chart recrider. Virtually all the expected neutral hellum lines were seen. The hellum ton line at $4686 \&$ was also present. Most of the remalning lines were 1dentified as due to B. few Impurities (axygen, hydrogen, carbon).

The strongest line was the $4471 \&$ neutral belium 1ine. The nearest line of any strength was one at $4437 \AA$ and even that was far less intense than the $4471 \&$ line. Almost all the light within a hundred-Angstrom band was found in the $4471 \&$ ilne. This was very convenfent, since in a multiple-beam spectrometer the monochromator was to be used w1th a very large slit, giving poor spectral resolut1on, whlle the interference which was to be produced required light with a fairly arrow ( 1 \&) spectrum. By tuning to the $4471 \&$ Ine the required coherence length could be obtained without prec1le spectrel resolution.

To estimate the intensities of various spectral components, 
a fow photon counting measurements were also made. For thls, the plasm was observed thraugh a $2 \times 2 \mathrm{~cm}$ aperture $50 \mathrm{~cm}$ away. The light was focused onto the entrance elit of a monochromator (25 $\mu \mathrm{x}$ $23 \mathrm{~mm}$, imaged to $68 \mu \times 8 \mathrm{~mm}$ within the plasma). Vartous spectral features were selected and the number of photocounts in 30 sec was recorded. The 4688 \& hellum fon line gave, after subtraction of beckground, 11.7 million counts, or $3.9 \times 10^{5}$ counts/sec. The 4471 \& neutral hellum line was not counted directly (In this setup it would have exceeded the counting speed of the equipment), but from integrated photocurrent messurements the $4686 \&$ line was found to be $0.8 \%$ as bright as the $4472 \&$ line. Thus the latter would have given a counting rate of $4.6 \times 10^{7} / \mathrm{sec}$.

As a check on these measurements, it was assumed that the phototube was $10 \%$ efficient and that the $4471 \AA$ line contained $10 \%$ of all the light emitted. Then an accounting of the total source volume and total solid angle gives an estimate of a few milliwatts of light emitted from the plasma. Judging by the apparent luminosity, this is a reasonable value. 


\section{E. Some liotes on the Definitions of Spectre}

\section{Direction-Dependent Spectra}

In discussfons of optical problems, the direction of emisstion or scattering of light is frequently defined (In $\underline{r}$-space) as the direction fram a localized source to a distant observer. (See, for example, P.ef.16.a.) In the present discussion, the directions of propagation of light waves bave been defined (In k-6pace) in terms of spatial Fourier transforms. The equivalence of the two descriptions should be noted.

In the $\underline{r}$-space formulation, one considers a source, $\underline{s}(\underline{r}, t)$, which is non-zero only vithin a bounded region--sey within a distance $r_{0}$ of an orlgin of coordinates. The emitted radiation is described by the ubual retarded Green's function integral,

$$
\left.\underline{E}(\underline{r}, t)=\int d^{3} r^{\prime} \frac{I}{\left|\underline{r}-\underline{\underline{r}}{ }^{\prime}\right|} \underline{E} \mid \underline{r}^{\prime}, t-\frac{\left|\underline{r}-\underline{r}^{\prime}\right|}{c}\right) .
$$

One assumes that $\underline{\underline{E}}(\underline{r}, t)$ is observed at a distant point $\underline{r}\left(|\underline{\underline{r}}| \gg r_{0}\right)$, where $\left|\underline{r}-\underline{r}^{\prime}\right|$ may be expanded,

$$
\left|\underline{r}-\underline{r}^{\prime}\right|=\left(|\underline{r}|^{2}-2 \underline{\underline{r}} \cdot \underline{\mathbf{r}}^{\prime}+\left|\underline{\underline{x}}^{\prime}\right|\right)^{1 / 2}=|\underline{\underline{r}}|-\hat{\mathbf{r}} \cdot \underline{\underline{r}}^{\prime}+\cdots
$$

Keeping only leading terms in the magnitude, but including first order corrections in the phase, the radiation field is then approximated.

$$
E(\hat{r},|\underline{r}|, t) \equiv \underline{E}(\underline{r}, t) \approx \frac{1}{|\underline{r}|} \int d^{3} r^{\prime} \underline{\underline{s}}\left[\underline{r}^{\prime}, t-\frac{1}{c}\left(|\underline{r}|-\hat{r}^{\prime} \underline{r}^{\prime}\right)\right] .
$$

At large distances $|\underline{\mathbf{r}}|$, the poynting vector is nearly parallel 
to $r$ and the energy flux (per unft solid angle) is simply

$$
I(\hat{r}, t)=\frac{c}{4 \pi}|\underline{r}|^{2}|\underline{E}(\hat{r},|\underline{r}|, t)|^{2} .
$$

Except for retardation, this should be independent of $|\underline{r}|$.

The spectral density is then defined in terms of the temporal Fourler transform of $\mathrm{E}(\hat{\mathrm{r}},|\underline{\mathbf{r}}|, \mathrm{t})$,

$$
I(\hat{r}, \omega)=\frac{c}{8 \pi^{2}}|\underline{r}|^{2}|\underline{E}(\hat{r},|\underline{r}|, \omega)|^{2},
$$

where

$$
\underline{E}(\hat{r},|\underline{r}|, \omega) \equiv \int d t e^{\perp \omega t} \underline{E}(\hat{r},|\underline{r}|, \hat{t}) .
$$

(We first consider only cases where this integral exists.) To compute the radiated energy, the intensity may be integrated in time or in f-yuency, since, according to Parseval's theorem,

$$
\int \mathrm{dt}|\underline{E}(\hat{r},|\underline{r}|, t)|^{2}=\int \frac{d \omega}{2 \pi}|\underline{E}(\hat{r},|\underline{r}|, \omega)|^{2}
$$

and hence

$$
\int \mathrm{dt} I(\hat{r}, t)=\int d \omega I(\hat{r}, \omega),
$$

when $I(\hat{r}, \omega)$ is normalized as in Eq. (E4).

Evaluating this spectral deselty,

$$
\begin{aligned}
I(\hat{r}, \omega)= & \frac{c}{8 \pi^{2}} \int d t \int d t^{\prime} e^{1 \omega t} e^{-1 \omega t^{\prime}} \int d^{3} r^{\prime} \int d^{3} r^{\prime \prime} \\
& \cdot \underline{s}\left[\underline{r}^{\prime}, t-\frac{1}{c}\left(|\underline{r}|-\hat{r} \cdot \underline{r}^{\prime}\right)\right] \cdot \underline{s}\left[\underline{r}^{\prime \prime}, t^{\prime}-\frac{1}{c}\left(|\underline{r}|-\underline{r} \underline{r}^{\prime \prime}\right)\right] .
\end{aligned}
$$

Subst1tuting

$$
\alpha \equiv t+\frac{l}{c} \hat{r} \cdot \underline{r}^{\prime}-\frac{|\underline{r}|}{c}
$$




$$
\alpha^{\prime}=t^{\prime}+\frac{1}{c} \hat{r} \cdot \underline{r}^{\prime \prime}-\frac{|\underline{x}|}{c}
$$

gives

$$
\begin{aligned}
& I(\hat{r}, \omega)=\frac{c}{8 \pi^{2}} \int d \alpha e^{\operatorname{lan} \alpha} \int d \alpha^{\prime} e^{-\operatorname{la} \alpha^{\prime}} \\
& \cdot \int d^{3} r^{\prime} e^{1(\omega \hat{r} / c) \cdot \underline{r}^{\prime}} \int d^{3} r^{\prime \prime} e^{-1(\omega \hat{r} / c) \cdot \underline{r}^{\prime \prime}} \underline{\underline{s}}\left(\underline{x}^{\prime}, \alpha\right) \cdot \underline{s}\left(\underline{\underline{x}}^{\prime \prime}, \alpha^{\prime}\right) \\
& \left.=\frac{c}{8 \pi^{2}}-\left(\frac{\omega \hat{r}}{c}, \omega\right) \cdot \underline{\underline{\varepsilon}} \mid-\frac{\omega \hat{r}}{c},-\omega\right) \\
& =\frac{c}{8 \pi^{2}} \underline{s}\left(\frac{\omega \hat{x}}{c}, \omega\right) \cdot \underline{s}^{*}\left(\frac{\omega \hat{r}}{c}, \omega\right) .
\end{aligned}
$$

where

$$
\underline{\underline{s}}\left|\frac{\omega \hat{\vec{r}}}{\mathrm{c}}\right|=\int \mathrm{d}^{3} r j d t \mathrm{e}^{-1\left|\frac{\omega \hat{r}}{\mathrm{c}} \cdot \underline{r}^{\prime}-\omega \alpha\right|} \underline{\underline{s}}\left(\underline{\underline{r}}^{\prime}, \alpha\right)
$$

Is the Fourler transform of $s\left(\underline{x}^{\prime}, \alpha\right)$. So, finally,

$$
I(\hat{r}, \omega)=\frac{c}{8 \pi^{2}}\left|\underline{s}\left(\frac{\omega \hat{r}}{c}, \omega\right)\right|^{2}
$$

Independent of $|\underline{r}|$ [as anticipated in the notation, $I(\hat{r}, \omega)]$. Hence, in the approximation of a localized source and a distant observer the definition in $\mathbf{g}$-space of the spectral density of the light enftted lato a given direction $\hat{\mathbf{r}}$ reduces to a simple expression invilving the Fourler transform of the distribution of sources.

In the present discusation, however, both the direction of propagation and the spectral density of a light wave have been described by spatial Fourfer trensforms. This is convenient 
because the transform of the fleld amplitude

$$
\begin{aligned}
\underline{E}(\hat{k},|\underline{k}|, t) & =\underline{E}(\underline{k}, t) \\
& \equiv \int d^{3} r e^{-\underline{\underline{k}} \cdot \underline{\underline{r}} \underline{\underline{g}}(\underline{r}, t)}
\end{aligned}
$$

defines in one step both a direction $\hat{k}$ and a waveleneth $2 \pi|\underline{k}|^{-}-$ [Here, as above, such expressions are considered well defined. If the simple Fourler integral does not converge, $\underline{E}(k,|\underline{k}|, t) \ldots$ and $\underline{E}(r,|\underline{r}|, \omega)$ discussed above--must be defined either as ensemble averages or as instantaneous spectra, as discussed in sect. 3 below. We consider first the simple case in which the usual definition of a Fourier transform is sufficient.] Using again the retarded Green's function $[\mathrm{Eq} .(\mathrm{El})]^{\dagger}$ to compute the field amplitude,

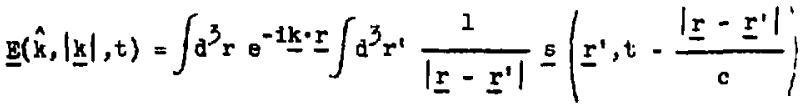

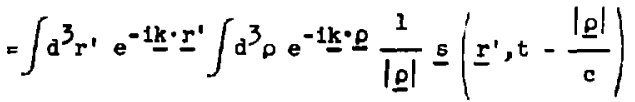

where

$$
\underline{\rho} \equiv \underline{\underline{r}}-\underline{r}^{\prime}
$$

If the $\underline{p}$ dependence 18 described in poler coordinates

$$
\left(d^{3} \rho+d|\underline{\underline{\rho}}||\underline{\underline{\rho}}|^{2} d^{2} \rho\right)
$$

the integration over directions may be done at once [as in Eq. (BII)] Leavin: 


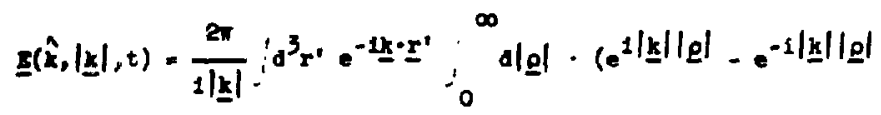

$$
\begin{aligned}
& \underline{i} \underline{r}^{\prime}, t=\frac{|\underline{g}|}{c} \\
& =\frac{2 \pi}{1|\underline{k}|} \int_{-\infty}^{t} d \tau \underline{s}(\underline{\underline{k}}, \tau)\left[e^{1|\underline{k}| c(t-\tau)}-e^{-1|\underline{\underline{k}}| c(t-\tau)}\right] \\
& \text { where } \tau \equiv t-\frac{|\underline{g}|}{c} \text {. }
\end{aligned}
$$

In the long time 1 inft, which, of course, is also required in Eq. (ES), we bave

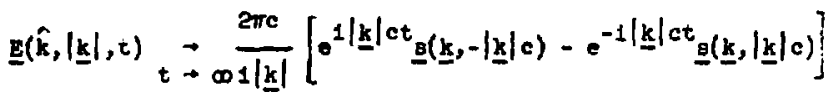

To use a k-space formulation, the spectral density must be expressed in terwo of $\underline{E}(\hat{k},|\underline{k}|, t ;$. Yet $\underline{E}(\hat{k},|\underline{k}|, t)$ is here seen to Involve two components of the source distribution, wh1le $I(\hat{x}, \infty)$, the spoctral density discussed above, was found to depend upon oniy a single component of $\underline{\mathrm{s}}$. Cloarly, the simple expression

$$
I(\hat{k},|\underline{k}|) \propto|\underline{k}|^{2}|\underline{E}(\hat{k},|\underline{k}|, t)|^{2}
$$

Is not equivalent to the usual frequency spectrus. The reeson for tbe difference is evident in Eq. (E8): $\underline{E}(\hat{k},|\underline{k}|, t)$ contains Degative as well as positive optical frequency components. The negative erequency componente, which propagate in the $-\hat{h}$ direction, would not be observed with a detector on the $+\hat{k}$ bide of the source. 
On the other hand, negative frequency components of $\underline{E}(-\underline{k})$, which would be observed, have not been included.

This suggests the form,

$I(\hat{k},|\underline{k}|) \propto|\underline{k}|^{2}\left[\left|\underline{E}^{(+)}(\hat{k},|\underline{k}|, t)\right|^{2}+\left|E^{(-)}(-\hat{k},|\underline{k}|, t)\right|^{2}\right]$

where by $\underline{E}^{(+)}(\hat{k},|\underline{k}|, t)\left[\underline{E}^{(-)}(\hat{k},|\underline{k}|, t) \mid\right.$ is meant the positive (negative) frequency portion of $\underline{E}(\underline{k}, t)$ :

$$
\begin{aligned}
& \underline{E}^{(t)}(\underline{k}, t) \equiv \int_{0}^{\infty} \frac{d \omega}{\delta \pi} e^{-1 \omega t} j_{-\infty}^{\infty} d t^{\prime} e^{i \omega t^{\prime}} \underline{E}\left(\underline{k}, t^{\prime}\right) \\
& \underline{E}^{(-)}(\underline{k}, t) \equiv \int_{-\infty}^{0} \frac{d \omega}{2 \pi} e^{-1 \omega u t} \int_{-\infty}^{\infty} d t^{\prime} e^{i \omega t^{\prime}} \underline{E}\left(\underline{k}, t^{\prime}\right)
\end{aligned}
$$

Equation (E9) can be further simplified, however, because the reality of $\underline{E}(\underline{r}, t)$ Implies that $\underline{E}(\underline{k}, \omega)=\underline{E}^{*}(-\underline{k},-\omega)$. Hence the two terms in the above expression are fdentical and only one is needed.

Th1s leaves

$$
I(\hat{k},|\underline{k}|) \propto|\underline{\mathbf{k}}|^{2}\left|\underline{E}^{(+)}(\hat{\mathrm{k}},|\underline{\mathbf{k}}|, t)\right|^{2} .
$$

Finalu, according to Eq. (E8),

$$
|\underline{\underline{k}}|^{2}\left|\underline{E}^{(t)}(\hat{k},|\underline{k}|, t)\right|^{2} \underset{t \rightarrow \infty}{\rightarrow} 4 \pi^{2} c^{2}|\underline{\underline{s}}(\underline{\hat{k}},|\underline{k}| c)|^{2}
$$

Which is time independent. Comparing this result with that obtalned for $I(r, w)[E q .(E 7)]$, the $r$ - and $\underline{k}$-spece formulations are oeen to be equivelent provided that (l) the direction $\hat{k}$ is identifled with the direction $\hat{r}$, (2) the wavelength $2 \pi|\underline{k}|^{-1}$ is related to the frequency $\omega$ by the usual, $\omega=|\underline{k}| c,(3) I(\hat{k},|\underline{k}|)$ is cor- 
rectly normalized, and (4) only positive frequency components of $\underline{E}(\hat{k},|\underline{k}|, t)$ are included.

The two expressions, $|\underline{g}(\omega \hat{\mathbf{r}} / \mathrm{c}, \omega)|^{2}$ and $|\underline{s}(\underline{k},|\underline{k}| c)|^{2}$, are still not quite equivalent, since the former includes negat ive freciuency components, while the latter does not, but this $1 \mathrm{~s}$ not an essential difference, because $\underline{g}(-\underline{k},-\omega)=\underline{s^{*}}(\underline{k}, \omega)$. Indeed, since $\omega$ appears in both the wave $r$ tor and the frequency of $\underline{s}(\omega \hat{r} / c, \omega)$, the two halves of the spectrum in Eq. (E7) simply correspond to the two terms in Eq. (E9).

The normalization of $I(\hat{k},|\underline{k}|)$ should be chosen to equate the Integrated spectrum to the total radiated enerey [as in Eq. (E6)]. The proper value is

$$
I(\hat{k},|\underline{k}|) \equiv \lim _{t \rightarrow \infty}\left(\frac{|\underline{k}|}{4 \pi^{2}}\right)^{2}\left|\underline{\underline{E}}^{(t)}(\hat{\mathrm{k}},|\underline{\mathrm{k}}|, t)\right|^{2},
$$

for then,

$$
\begin{aligned}
\int_{-\infty}^{\infty} d \omega I(\hat{r}, \omega) & \int_{-\infty}^{\infty} d \omega \frac{c}{8 \pi^{2}}\left|\underline{s}\left(\frac{\omega r}{c}, \omega\right)\right|^{2} \\
& =\int_{0}^{\infty} d \omega \frac{c}{4 \pi^{2}} \mid \underline{s}\left(\frac{\omega r}{c}, \omega||^{2}\right. \\
& =\int_{0}^{\infty} \operatorname{cd}|\underline{k}| \frac{c}{4 \pi^{2}}|\underline{s}(\underline{k},|\underline{k}| c)|^{2} \\
& =\int_{0}^{\infty} d|\underline{k}| \frac{1}{\left(4 \pi^{2}\right)^{2}}\left[\underline{t \rightarrow \infty}\left|\underline{\underline{E}}{ }^{(+)}(\hat{k},|\underline{k}|, t)\right|^{2}\right]
\end{aligned}
$$




$$
=\int_{0}^{\infty} \mathrm{d}|\underline{\underline{k}}| I(\hat{k},|\underline{k}|)
$$

as required. The normalization of the two intensities is most trensparent from a comparison if differentials,

$$
\begin{aligned}
& I(\hat{r}, \omega) d \omega d^{2} \hat{r}=\left.\frac{c}{4 \pi}\left|\frac{d \omega}{2 \pi}\right\rangle^{\mid}|\underline{r}|^{2} d^{2} \hat{r}|| \underline{E}(\hat{r},|\underline{r}|, \omega)\right|^{2} \\
& I(\hat{k},|\underline{k}|) d \underline{\underline{k}}\left|d^{2} \hat{k}=(2)\left(\frac{I}{4 \pi}\right)\right| \frac{d|\underline{k}|}{2 \pi} \mid\left[|\underline{k}|^{2} \frac{d^{2} \hat{k}}{(2 \pi)^{2}}\right]_{t \rightarrow \infty} \lim _{t \rightarrow \infty} \\
& \left|\underline{E}^{(+)}(\hat{k},|\underline{k}|, t)\right|^{2}
\end{aligned}
$$

[Again, the factor of two in $\pi(\hat{k},|\underline{k}|)$ reflects the fact that this epectral density is non-zero only for positive frequencies, $|\underline{x}| c>0$.

Th1s definition is in accord with si idard practice. In anlyses of optical problems, the light Intensities and correlation runctions are often defined in terms of the positive frequency portion of the radiation field. ${ }^{2 c}$ In an -space formulation, $E(\underline{r}, t)$ is roel and $\underline{E}^{(+)}(\underline{\underline{r}}, t)$, whish is called the associated anabytic olgnal, is a complex valued quantity wh1ch completely deecribes the fleld $\underline{E}(\underline{r}, t)$. The use of an anazytic signal to a cenomization of the familiar device of replacing a cosine by - conplex exponential. If $\underline{E}(\underline{r}, t) 18$ nearly monochromatic, the wonttude of the analytic algnal $\left|\underline{\mathrm{E}}^{(+)}(\underline{x}, t)\right|$ ia a slowly varying quentity.

The magnitude of the positive frequency portion of the apatial Fourler trensform $\left|\underline{\underline{g}}{ }^{(t)}(\underline{k}, t)\right|$ which was used above also contains no 
rapid osctllation. [ See Eq. (EB). There elther term alone has constant monitude, but their aum does not.j This elimination of the rapid optical frequency variation is convenient becsuse it permits one to define a slowly varying or constant light intensity without resort to a time averaging procedure.

However, $\underline{\underline{E}}^{(+)}(\underline{k}, t)$ was introduced above for a different reason: to define a direction of propagation of the wave. The definition of an optical spectrum $I(\hat{k},|\underline{k}|)$ in terms of the spatial Fourler transficm of the fleld requires the use of only the positive frequency portion of $\underline{E}(k, t)$. If negative frequencles were included, the results would not be equivalent to the frequency spectrum $I(\hat{r}, \omega)$ of the light emitted in the $\hat{k}=\hat{r}$ direction. Yet the quantilj $\underline{E}^{(+)}(\underline{k}, t)$, which was seen to be needed for this, is JW .t the transform of the usual analytic signal $\underline{E}^{(+)}(\underline{r}, t)$ since. at least for well behaved functions, the two operations commute:

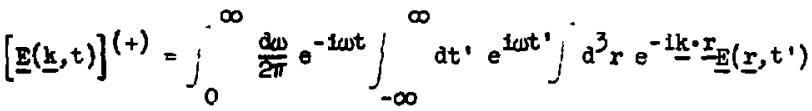

$$
\begin{aligned}
& =\int d^{3} r e^{-1 \underline{k}} \cdot \underline{r} \int_{0}^{\infty} \frac{d \omega}{2 \pi} e^{-1 \omega t} \int_{-\infty}^{\infty} d t \cdot e^{1 \omega t^{\prime}} \underline{E}(\underline{r}, t) \\
& =\int d^{3} r \theta^{-1 k} \cdot \underline{r} \underline{E}^{(+)}(\underline{r}, t)
\end{aligned}
$$

It should be noted also that different definitions of the anlytic signal are in uee. Save authors ${ }^{2 c}$ consider $E^{(+)}(\underline{r}, t)$, while others ${ }^{1 a}, 36$ fine an analytic aignal equal to twice this quantity [to elsminate the ractor of two in Eq. (E14) below]. A 
superscript r--for "real"--is scmetimes used to identify the original slgnal, but this is inappropriate when spatial Fourler transformo are employed, since $\underline{\underline{E}}(\underline{\underline{k}}, t)$ is not necessarily a real quantity. Th: vughout the present discussion, a superscrfpt $(+)$ 1s used to identify the analytic signal, as defined by Eq. (ElO) [and similarly for other quantities, $B^{(+)}, \xi^{(+)}, \underline{E}^{(+)}(\underline{r}, t)$, etc. $]$. Since $\underline{E}(\underline{r}, t)$ Is real, Eq. (ELO) Implies that

$$
\underline{E}(\underline{r}, t)=2 \mathrm{He} \underline{\underline{E}}^{(+)}(\underline{\underline{x}}, t) \text {. }
$$

In $k$-space, however, both $\underline{E}(\underline{k}, t)$ and $\underline{E}^{(+)}(\underline{k}, t)$ are, in general, complex valued functions. For such functions, parseval's theorem Implies that

$$
\int_{-\infty}^{\infty} d t|\underline{\underline{E}}(\underline{k}, t)|^{2}=\int_{-\infty}^{\infty} d t\left|\underline{\underline{E}}{ }^{(-)}(\underline{k}, t)\right|^{2}+\int_{-\infty}^{\infty} d t\left|\underline{\underline{E}}^{(+)}(\underline{k}, t)\right|^{2} .
$$

For a real valued function such as $\underline{E}(\underline{r}, t)$, this reduces to

$$
\int_{-\infty}^{\infty} d t|\underline{E}(\underline{r}, t)|^{2}=2 \int_{-\infty}^{\infty} d t\left|\underline{\underline{E}}{ }^{(+)}(\underline{r}, t)\right|^{2} \text {. }
$$

Using this rolation, the time-averaged intensity of polychromatic as vell as monochromatic light can be expressed in terms of the bosociated analytic signal.

The analyais of optical interference, however, requires an exprossion for the replaly varying amplitude, as well as the mean Intonsity, of the interfering waves. Both the arolftude and the Intene1ty of the I1ght transmitted by a spectrometer may be expresed in terms of the Fourler transform of the 1nput, as can be 
seen from an abalysis of the actual effect of such an instrument. A brief review of this discuselon is included here.

In a grating spectrometer, the length of the path from the entrance slit to the exit slit is different for light reflected from different lines of the greting. Components of the light are delayed by different amounts and the output of the instrument is a euperposition of many preceding inputs.

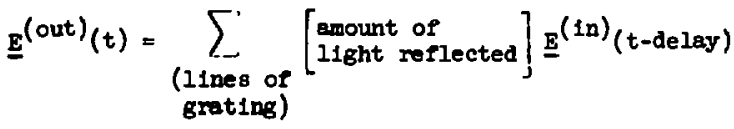

$$
\begin{aligned}
& =\int_{0 \text { (delay time) }}^{\infty} d \tau f(\tau) \underline{E}^{(1 n)}(t-\tau) .
\end{aligned}
$$

$f(\tau)$ is a real valued quantity which, according to Eq. (E14) may be expressed in terms of an associated analytic signal. Making th1s replacement,

$$
\begin{aligned}
\underline{E}^{(\text {out })}(t) & =2 \operatorname{Re} \int_{0}^{\infty} d \tau f^{(t)}(\tau) \underline{E}^{(\operatorname{In})}(t-\tau) \\
& =2 \operatorname{Re} \int_{-\infty}^{t} d(t-\tau) \int_{0}^{\infty} \frac{d \omega}{2 \pi} e^{1 \omega(t-\tau)} e^{-1 \omega t} f(\omega) E{ }^{(1 n)}(t-\tau) \\
& \rightarrow 2 \operatorname{Re} \int_{0}^{\infty} \frac{d \omega}{2 \pi} e^{-1 \omega \omega t} \tau(\omega) \underline{E}^{(\ln )}(\omega)
\end{aligned}
$$

Which just describes the effect of the spectrometer in terms of a trensfer tunction $f(\omega)$.

In the limit of perfect resolution, that is, of an infinite 
s1nusoldal grating, $f(\tau)=\cos \omega_{0} \tau, f(\omega)=\pi \delta\left(\omega-\omega_{0}\right)+\delta\left(\omega+\omega_{0}\right)$, and Eq. (E16) reduces to

$$
\underline{E}^{(\text {out })}(t) \underset{t \rightarrow \infty}{\rightarrow} e^{-1 \omega_{0} t} \underline{E}^{(\text {ln })}\left(\omega_{0}\right) \text {. }
$$

To calculate the mean intensity of th1s, only the magnitude $\left|\underline{E}\left(\omega_{0}\right)\right|$ is needed, since, after time averaging,

$$
I=\frac{c}{4 \pi} \overline{\left|R e e^{-1 \omega_{0} t} \underline{E}\left(\omega_{0}\right)\right|^{2}}=\frac{c}{8 \pi}\left|\underline{E}\left(\omega_{0}\right)\right|^{2} .
$$

To describe interference, however, the rapid osclilation of the field must also be considered. It is again useful to express the result both in terms of vave vector $\underline{k}$ and in terms of direction $\hat{r}$ and frequency w. Constderting once more a locallzed source, $a$ diatant observer, find a temporal Fourler transform, $\underline{\mathbb{E}}(\hat{\boldsymbol{r}},|\underline{\underline{r}}|, \infty)$, we have from Eq. (El6) an expression for the amplitude,

$$
\underline{E}^{(\text {out })}(t) \propto \operatorname{Re}\left[|\underline{r}| e^{-1 \omega t} \underline{\underline{E}}(\hat{\boldsymbol{r}},|\underline{\underline{r}}|, \omega)\right] .
$$

The factor of $|\underline{r}|$ permits normalization of the intensity to solid angle as was done with $I(\hat{r}, \omega)$ in Eq. (E4).

The same amplitude can also be expressed in terms of the apatial Fourier transform of the field and the two forms can be related to the source density as was done with $I(\hat{k},|\underline{k}|)$ and $I(\hat{x}, \omega)$. In exactly the same manner as in that discussion, one obtains the two equations

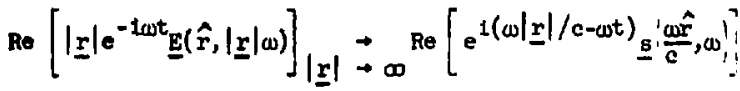




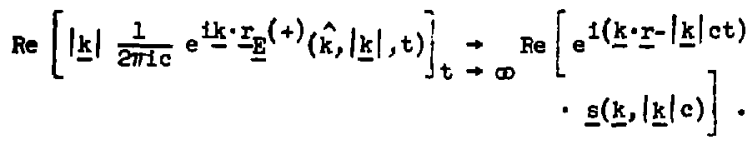

Elther of these forms can be used to describe the rleld amplitude. The normalization, if needed, can be obtained from the correspondIng expression for the spectral density, Eq. (E13a) or (E13b). If the form of (EITb) is used, It should be noted that the order of the operations is important, since $(t)$ and (Re) do not commute. 2. A Property of the Analytic S1gnal

Equation (E14) implies that

$$
\left[\operatorname{Re} \underline{E}^{(+)}(\underline{r}, t)\right]^{(+)}=\frac{1}{2} \underline{E}^{(+)}(\underline{r}, t) .
$$

Equation (E14) applies only to real valued functions, $\underline{\underline{E}}(\underline{\underline{r}}, \mathrm{t})$, but the relation (EIB) is in fact more generally valld. Because this result is used in chapter II, a simple derivation is included here. Consider a complex valued quantity, $\underline{E}(\underline{k}, t)$. The positive erequency portion, $\underline{E}^{(+)}(\underline{k}, t)$, may be written

$$
\underline{E}^{(t)}(\underline{k}, t)=[\operatorname{Re} \underline{E}(\underline{k}, t)]^{(+)}+1[\operatorname{In} \underline{E}(\underline{k}, t)\}^{(+)} \text {. }
$$

Re $\underline{E}(\underline{k}, t)$ and $I m \underline{E}(\underline{k}, t)$ are both real valued quantities, and hence a relation $11 k$ (E14) must hold for elther term alone. Th1s suggests that even though $\underline{\underline{E}}(\underline{k}, t)$ is clearly not equal to the real part of $2 \underline{E}^{(+)}(\underline{k}, t)$, the imeginary part of $\underline{\underline{E}}^{(+)}(\underline{k}, t)$ way still be redundent.

Taking the resl part, 
$-250-$

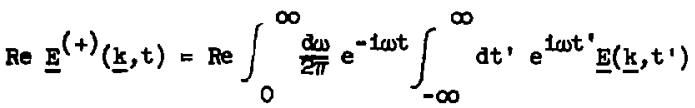

$$
\begin{aligned}
& =\int_{0}^{\infty} \frac{d \omega}{2 \pi} \int_{-\infty}^{\infty} d t^{\prime} \frac{1}{2}\left[\underline{E}\left(\underline{k}, t^{\prime}\right) e^{-1 \omega\left(t-t^{\prime}\right)}\right. \\
& \left.+\underline{E}^{*}\left(\underline{k}, t^{\prime}\right) \mathrm{e}^{+1 \omega 0\left(t-t^{\prime}\right)}\right] \text {. }
\end{aligned}
$$

The positive frequency portion of this,

$$
\begin{aligned}
& \operatorname{Re} \underline{\underline{E}}^{(+)}(\underline{k}, t){ }^{(+)}=\int_{0}^{\infty} \frac{d \omega}{2 \pi} e^{1 \omega t} \int_{-1}^{\infty} t^{\prime} \cdot e^{l \omega t} \int_{0}^{\infty} \frac{d \omega^{\prime}}{2 \pi} \int_{-\infty}^{\infty} d t " \\
& \text { - } \frac{1}{2}\left[ \pm=\left(2, t^{\prime \prime}\right) \mathrm{e}^{-t \omega^{\prime}\left(t^{\prime}-t^{\prime \prime}\right)}\right. \\
& +E^{*}\left(\underline{k}, t^{\prime \prime}\right) e^{+1 \omega^{\prime}\left(t^{\prime}-t^{\prime \prime}\right) ? !} \\
& =\int_{0}^{\infty} \frac{\partial \omega}{2 \pi} e^{-1 \omega t} \int_{0}^{\infty} \frac{\partial \omega^{\prime}}{2 \pi} \int_{-\infty}^{\infty} d \tau \int_{-\infty}^{\infty} d t^{\prime \prime} \frac{I}{2} e^{1 \omega t "} \\
& \text { - }\left[\underline{E}\left(\underline{k}, t^{\prime \prime}\right) e^{1\left(\omega-\omega^{\prime}\right) \tau}+\underline{E}^{*}\left(\underline{k}, t^{\prime \prime}\right) e^{1\left(\omega+\omega^{\prime}\right) \tau}\right] \\
& =\int_{0}^{\infty} \frac{d \omega}{2 \pi} e^{-1 \omega t} \int_{0}^{\infty} \frac{\alpha \omega^{\prime}}{2 \pi} \int_{-\infty}^{\infty} d \tau \frac{1}{2} \\
& \text { - }\left[\underline{\underline{E}}(\underline{\underline{k}}, \omega) \mathrm{e}^{1\left(\omega-\omega^{\prime}\right) \tau}+\underline{\underline{E}}(\underline{\underline{k}},-\omega) \mathrm{e}^{1\left(\omega+\omega^{\prime}\right) \tau}\right] \text {, }
\end{aligned}
$$

where $\tau \equiv t^{\prime}-t^{\prime \prime}$. The $\tau$ integration gives

$$
\int_{0}^{\infty} \frac{d \omega}{2 \pi} e^{-1 \omega t} \int_{0}^{\infty} \frac{d \omega^{\prime}}{2 \pi} \frac{1}{2}\left[\underline{E}(\underline{k}, \omega) 2 \pi 8\left(\omega-\omega^{\prime}\right)+\underline{E}(\underline{k},-\omega) 2 \pi 8\left(\omega+\omega^{\prime}\right)\right] .
$$

Since the $\omega$ and $\omega^{\prime}$ integrations include only positive frequencies, the second term contributes nothing $[\underline{E}(\underline{k}, \omega)=0$ at $\omega=0]$, and 
the elrst gives

$$
\int_{0}^{\infty} \frac{d \omega^{\prime}}{2 \pi} 2 m \delta\left(\omega-\omega^{\prime}\right) \underline{E}(\underline{\mathbf{k}}, \omega)= \begin{cases}\underline{\underline{E}}(\underline{\mathbf{k}}, \omega), & \omega>0 . \\ 0, & \omega<0 .\end{cases}
$$

Using this, we have

$$
\begin{aligned}
{\left[\operatorname{Re} \underline{E}^{(t)}(\underline{k}, t)\right]^{(t)} } & =\int_{0}^{\infty} \frac{d \omega}{2 \pi} e^{-1 \omega t} \frac{1}{2} \underline{E}(\underline{k} \omega) \\
& =\frac{1}{2} \underline{E}^{(+)}(\underline{k}, \omega) .
\end{aligned}
$$

This result, identical in form to Eq. (E18), applies to complex valued functions $\underline{E}(\underline{k}, t),[1 . e .$, to any well behaved complex valued function of time. We beve assumed only that simple algebrafe steps are valid, the the integrals used exist, and thet there is no contribution from $\omega=0$.

The ame result can be obtained by evaluating separately the contributions of the two terms in Eq. (E19).

\section{Time Dependent Spectra}

The use of Fourler iransforms to describe a spectroscopic messurement is convenient for analys18, but such a description is highly Ideal'zed. Literally, the measurement of any wave vector component, $\underline{E}(\underline{k}, t)$, of a fleld, $\underline{E}(\underline{r}, t)$, requires observation of $\underline{E}(\underline{r}, t)$ at every point in space. A description in terms of frequency components requires knowledge of the fleld throughout all time. A long time limit is also needed in the k-spece analysis [c.f., Eq. (I.7) or Eq. (II.15)].

The output of a real epectrometer is not a single apectral 
amplitude; it is a time-varying signal which depends upon the past, but not the future input to the instrument. This situation is not unique to optics. Any actual spectral measurement is related only In a long time limit to a simple Fourler transform. To improve upon this description, otber matbematical techniques have been developed (primarily for ttudies of electrical signais in cormunication systems) which better represent the effect of a spectrometer.

Most simply, the usual Fourier transform $f(\omega)$ of a signal $f(t)$ Is sometimes replaced by an 1ntegral over past, but not future times. The result is a time dependent quantity which is cailed a Iunning spectrum: 60

$$
f(\omega ; t) \equiv \int_{-\infty}^{t} d t^{\prime} e^{1 \omega t^{\prime}} f\left(t^{\prime}\right) .
$$

This definition can be further modified to reduce or elimtnate the effect of events in the distant past. By 1ntegrating over only a finite interval or by Including an appropriate weighting factor in the integrand, one obtains a time localized expression which is called an instantaneous spectrum. 60 An instantaneous spectrum not only better represents the effect of a real spectral Instrument, it has the added advantage of being well defined for many functions whose Fourler transform does not exfst at all.

The analyses in this report have all been done with reference only to a simple Fourfer transform, but much the same discussion could be done in terms of time-dependent spectra. Indeed, expresalons for ruaning spectra and for instantaneous spectra emerge in 
a simple and atralghtforward way from calculations done in Chapters I and II. For this reason it seems appropriate at least to wention how these concepts could be introduced in this discussion. Exprestions of the form of Eq. (E21) appeared both in the anlysis of scattering in Chapter I [see Eqs. (I.7) and (B7), frow which it canes] and in the aralyols of a two-beam spectrometer in Chepter II [see Eq. (II.15) and the discussion after Eq. (BII)]. In each case a long time limit was invoked to change the result Into a standard Fourier integral. But one could instead consider the expression as a running spectrum. So one need not introduce th1s concept--it is already prosent in the calculations as they stand.

Both of the same celculations started with a spatial Fourier transform, which involves an integral of the fleld over all space. since this represents the effect of the diffraction grating in the opectraweter, it would be more realistic to replace the Fourler integrel by one over a rinite volume camparable to the dimensions of the greting. If this were done, the final expressions fust discused [Eq. (B7), etc.] would have a finfte lower limit too. (The integrals over the retarded times cave from integrals over $|\underline{\underline{g}}|=|\underline{\underline{x}}-\underline{\underline{r}}|$, which cameoriginally from the opatial fourier transforms.) Thus a more realistic model of the spectraneter would Give at once an instantaneous spectrum as the output.

In Chapters I and II, the time dependence of the measured spectra, $I_{1,2}(\omega ; t)$, and of the corresponding sources ${ }^{\prime}\left(\omega ; \underline{r}^{\prime}, t\right)$ was reintroduced after the Fourier transformation, essentially be tresting the two time scales separately. If instantsneous spectra 
ware explicitiy employed, this separation of time seules would not be necessary. The difficulty with such an approach 18, of course, that it would not permit use of the standard theory of Fourier transformation. In particular, the light Intensity, which was defined by integration over the complete timo axis would then have to be restated locally. (Recall that the intensity was written in terme of an analytic signal, which was in turn defined in terms of the whole Fourfer trangformatior.) Also, the treatwent of the total Intensity as just an integral over |k|k| components [In Eqs. (II.8) and after] would have to be rewritten for a local definition of Intensity. Thus a conversion to an Instantaneous spectral forwulation would require now definition of intensity, and protebly lso of analytic signals.

Pinally, it should be noted also that the concept of a timedependent spectrum 1s closely related to the corcept of a timedependent correlation function. (Here "time" means the instant of observation, not the length of a delay.) Both a scattering experiment snd a simple two-beam spectroscopic apparatus vould Give a measurement of one Fourfer component of the fluctuations In the source or electron denefty within a plasma. Accoriling to the Wiener Khintehine theorem ${ }^{19}$ [see Eq. (I.14)], this gives information about the two-point correlation function of the source or electron diatribution. This correlation function is customarily defined in terms of a stationary ensemble of systems. But any real observetion is made on a single system, and most experiments 1nvolve pulsed pleamas which are far from stationary. These 
differences are just as besic as--and, in fact, related to--the difrerence between a four'er transform and a measured spectrum. It is possible, bovever, to defl.je a correlation function which does not aseume a stationary situation. The correlation function, like the spectrum, can also be defined in terms of a tise-localized expression. Several authers 61 have used definitions of this sort to generalize the Wiener-Khintchine theorem--obtaining a relation between this type of correlation function and a corresponding instantaneous power spectrum. Thus a more real1stic treatment of the correlation function leads again to the 1dea of a time-depencent spectrum. Conversely, description of the radiation from $a$ plesw in terms of instantaneous spectra--a description which emerges neturally from a more realistic model of the optical devices used--would lead to a description of the plasma in terme of time-localized expresatons for the correlations between particles. 


\section{F. Notation}

The Fourler transform is used repeatedly in this discussion. For any function, such as $\xi(\underline{r}, t)$, the spatial fourier transform 1s denoted by $\xi(\underline{k}, t)$, while $\xi(\underline{\underline{I}}, \omega)$ denotes the temporal Fourier transform. [For the definition used, see Eq. (1.8).] For anv function of time, such as $\xi(\underline{r}, t)$ or $\underline{E}(\underline{\underline{r}}, t)$, a superscript $(+)$ is used to denote the positive frequency (or analytic signal) portion, $\xi^{(+)}(\underline{r}, t), \underline{E}^{(+)}(\underline{r}, t)$, etc. [Th1s is defined by Eq. (ElOa) in Appendix $E . j$

A wave amplitude which is linear in the source may be divided into separate contributions from the different source points. This is occasionally indicated by a second spatial variable, as in $\xi\left(\underline{r}, t ; \underline{r}^{\prime}\right)$ which means the contribution to $\xi(\underline{r}, t)$ from sources near the point $\underline{x}^{\prime}$.

A vector quantity is denoted by an underscore, as $\underline{r}$, Iís magnitude by the addition of an absolute value sign, as $|\underline{r}|$, and 1 ts direction by the same symbol with circumflex, as $\hat{r}$. Thus $\underline{r}=|\underline{r}| \hat{\mathbf{r}}$, $\underline{E}=|\underline{E}| \hat{E}$, etc.

Strictly speaking temporal fourier transformed quantities are functions of frequency and do not depend ois time. But in practice, measured spectra are time varying, a fact which cannot always be ignored. In our notation, a time dependence is explicitly indicuted in such quant1tie: as $\dot{\alpha}\left(\omega ; \underline{r}^{\prime}, t\right)$ in those equations where it is important. (The mathematical besto of this procedure is diecussed in Appendix E.3.) 
The principal symbols used in the discussion are:

a.

$$
a\left(\underline{r}^{\prime} t\right)
$$

토

a'

$\underline{A}$

b

$\underline{B}$

c

$c_{A}, c_{B}, c_{O}, \tilde{c}, c_{O}^{\prime}$

$c_{n_{e}}(\tau)$

d

d

D

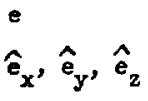

$\underline{E}$

$\underline{E}_{1}$

$\underline{E}_{0}$

$E_{0}^{1}$ angular spread of a beam (in Sect. I.B)

amplitude of a neerly monochrometic source

[see Eq. (II.36)]

accelesution

acceleration of a particle at time $t^{\prime}$ (In

sect. I.C)

magnetic vector potential

apparent angular size of a focus (in sect. I. B)

magnetic field strength

speed of light

(uzed in Sect. II.5.3) various time delays

in mult1ple-beam spectrometers

electron density time correlation function

lateral displacement of the extraordinary ray

in a calcite rhomb

a difference in optical path of one wave-

length (in Fig. I-4b)

the length of a calcite rhomb

electronic charge

unit vectors of a Carteglan coordinate system

electric field strength

electric field of an incident light wave (in

a scattering experiment)

amplitude of an incident light wave, $E_{1}$

$=\left(\underline{I}_{\mathrm{I}}-\hat{\mathrm{k}}_{\mathrm{s}} \hat{\mathrm{k}}_{s}\right) \underline{E}_{0}$, the component of $\underline{E}_{0}$ normal to $\underline{k}_{s}$ 
$\underline{\mathbf{E}}_{\mathrm{g}}$

$$
f_{1}, f_{2}
$$

$f(\omega)$

$F\left(y^{\prime}\right)$

$\mathrm{g}_{\mathrm{A}}, \mathrm{g}_{\mathrm{B}}$

I

$I(\hat{k},|\underline{k}|)$

$I(\hat{\gamma}, \omega)$

$I_{0}$

$I_{1}(t), I_{2}(t)$

1

$\underline{k}_{A}, \underline{k}_{B}$

$\underline{i}$

$\mathbf{k}_{\mathbf{s}}$

$\mathbf{s}$

L elestric fleld of a scattered wave

focal lengths of the input and output lenses

of m multiple-beam spectrometer (see FIg. II-6)

the (complex valued) transfer function of a

spectrometer or spectral illter

a function which defines the region observed

by a multiple-beam spectrometer [see Eq. (II.28)]

Green's functions which describe the central

section of a multiple-beam spectrometer [ see

Eq. (II.2I)]

the unit tensor of the second rank

the spectral density of a ligint wave defined

In terms of spatial Fourfer transforms

the spectral density of light emitted in

direction $\hat{\mathbf{r}}\left[\right.$ see Eq. ( $\left.\left(\mathrm{L}^{4}\right)\right]$

the intenfity of an incident light weve

the two light intensities which are measured

in a multiple-beam spectrometer

electric current density

wave vectors of light in beams $A$ and $B$

wave vector of an Incident light wave

wave vector of an observed scattered wave

a difference wave vector $\left(=\underline{k}_{s}-\underline{k}_{i}\right.$ or

$\left.k_{B}-\underline{k}_{A}\right)$

the length of a collimator

mase of a perticle 


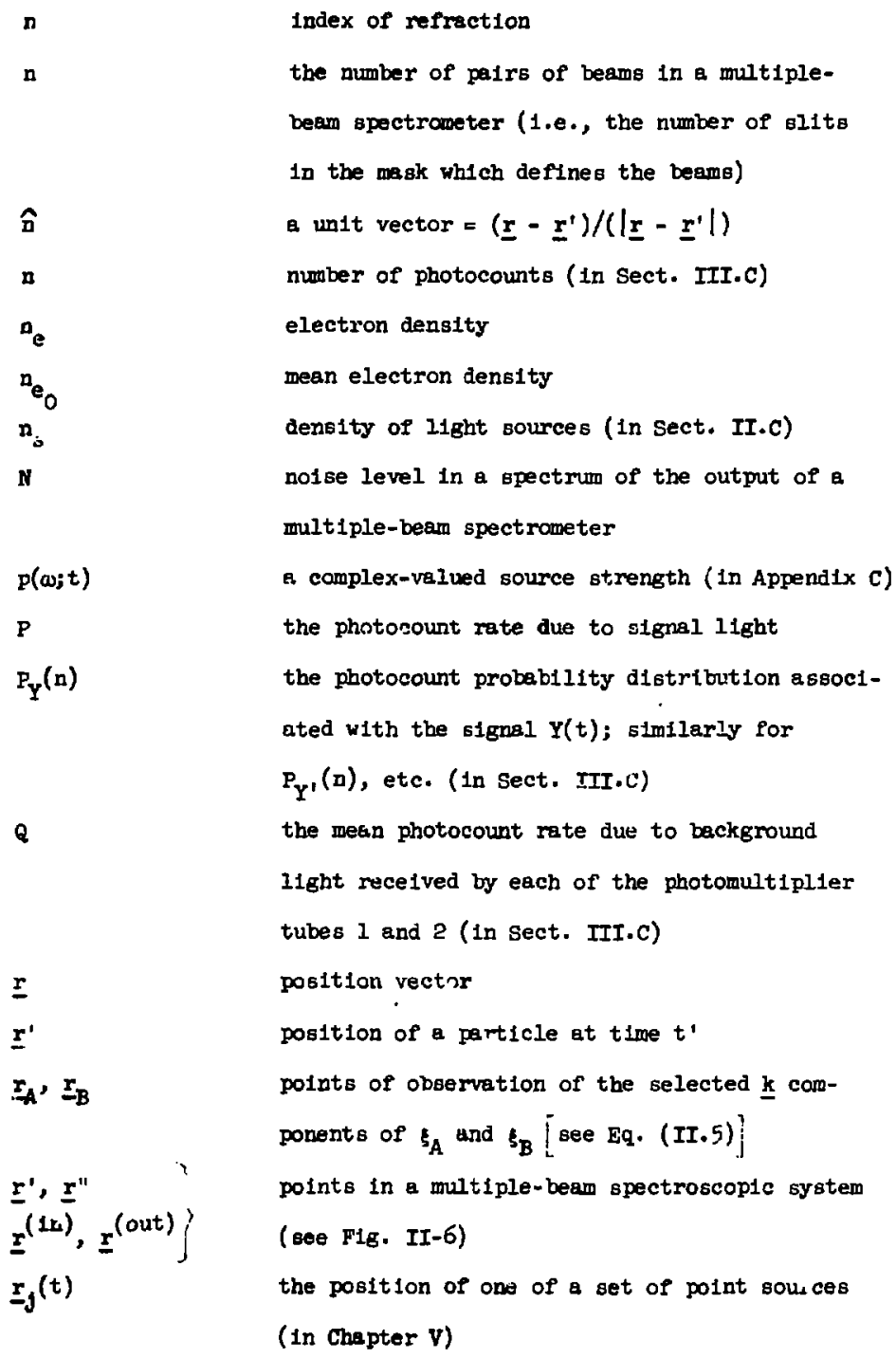

I

n

บ

$\mathbf{n}$

a

a

$n_{5}$

1)

$p(\omega ; t)$

P

$\mathrm{P}_{Y}(\mathrm{n})$

Q

$\underline{I}$

$\underline{I}^{\prime}$

$\underline{x}_{A}, \underline{r}_{B}$

$\underline{r}^{\prime}, \underline{r}^{\prime \prime}$

$\left.\underline{r}^{(\text {Ir) }}, \underline{r}^{\text {(out) }}\right\}$

$\underline{x}_{j}(t)$

Index of refrection

the number of pairs of beams in a multiplebeam spectrometer (1.e., the number of slits In the mask which defines the beams)

a unit vector $=\left(\underline{r}-\underline{r}^{\prime}\right) /\left(\left|\underline{r}-\underline{r}^{\prime}\right|\right)$

number of photocounts (In Sect. III.c)

electron density

mean electron density

density of light sources (In Sect. II.c)

noise level in a spectrum of the output of a

multiple-beam spectrometer

e. complex-valued source strength (in Append 1x c)

the photosount rate due to slgnal light

the photocount probability distribution assoc1-

ated with the signal $Y(t)$; similarly for

$P_{Y^{\prime}}(n)$, etc. (In sect. III.C)

the mean photocount rate due to background

ilght recelved by each of the photomultiplier

tubes 1 and 2 (in sect. III.C)

position vector

position of a particle at time $t$ '

polats of observation of the selected $\underline{k}$ com-

ponents of $s_{A}$ and $s_{B}[$ see Eq. (II.5) $]$

points in a multiple-beam spectroscopic system

(see Fig. II-6)

the position of on of a set of point sousces

(In Chapter V) 


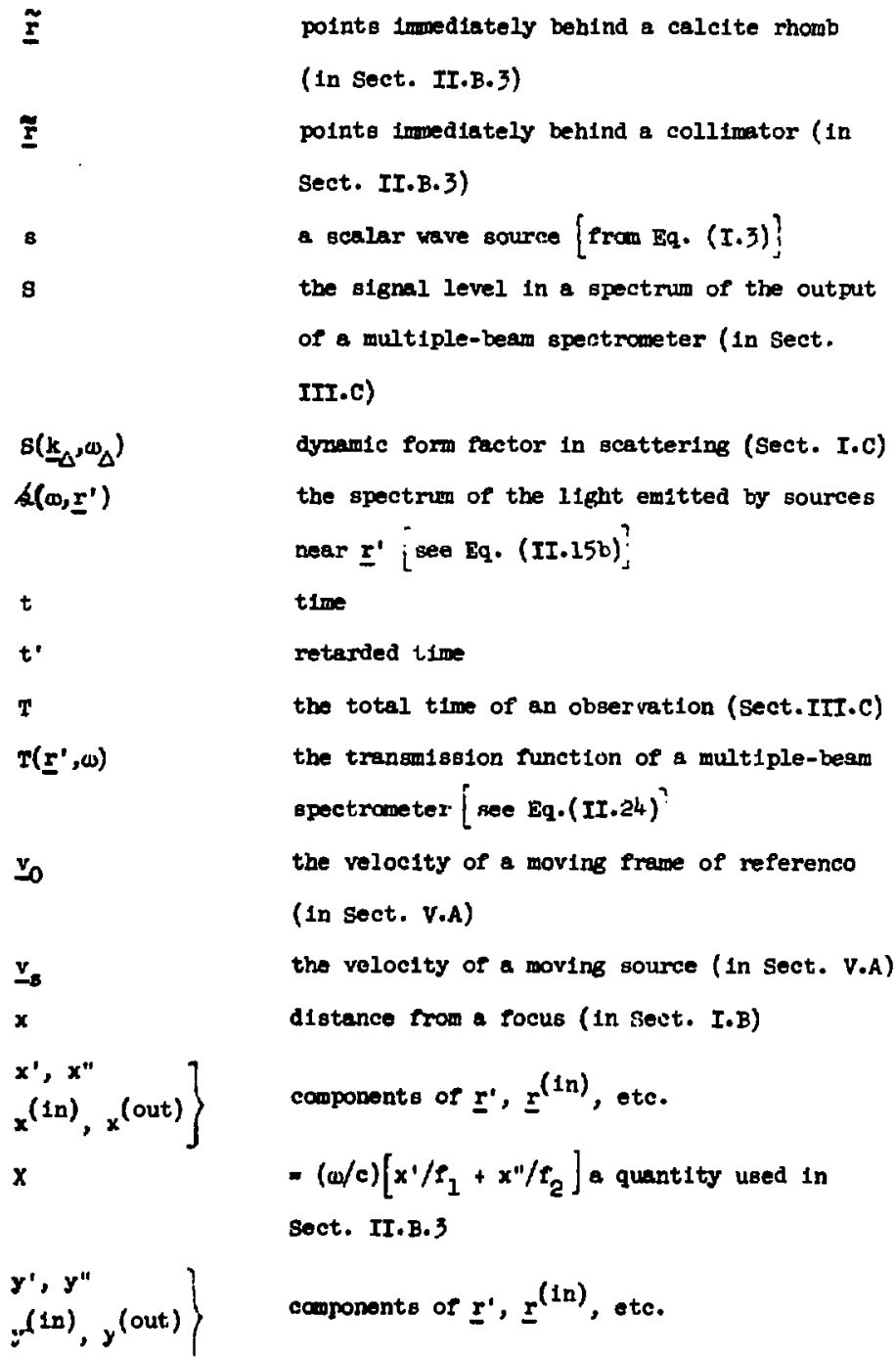


$\mathbf{Y}(\mathbf{t})$

$Y^{\prime}(t)$

$Y_{1}(t), Y_{2}(t)$

$\mathbf{Y}$

$z^{\text {(1n) }, z^{\prime \prime}}$ (out) $\}$

$z(t)$

$\alpha$

$\alpha$

B

$\tilde{r}_{\mathrm{BA}}(\tau,|\underline{k}|)$

$\Gamma_{\mathrm{BA}}\left(\boldsymbol{r},|\underline{\mathbf{k}}|, \underline{\underline{r}}^{\prime}\right)$

$\Gamma(\mathbf{n}, \mathbf{n})$

$\Gamma_{\mathrm{BX}}(\tau,|\underline{\mathbf{k}}|)$

$r_{B A}\left(\tau,\left|\underline{k}_{A}\right|,\left|k_{B}\right|\right)$ the output signal from a multiple-beam apectrometer

the result of frequency mixing and then time averaging the signal $Y(t)$ (In Sect. III.C) the positive and negative portions of $Y^{\prime}(t)$ (in sect. III.C)

$=(a / c)\left[y^{\prime} / f_{1}+y^{\prime \prime} / f_{2}\right]$

components of $\underline{r}^{1}, \underline{x}^{(i n)}$, etc.

$=\left[Y^{\prime}(t)\right]^{2}$ (In Sect. III.c)

scattering parameter $=1 /\left(\left|\underline{k}_{\Delta}\right| \lambda_{I}\right)$

the angle at which two observed beams intersect at a source

the angle eubtended at the source by a single observed boam

the mutual coherence between the $|\underline{k}| c$ froquency component of the light in beams $A$ and $B$ [ $800 \mathrm{Eq}$. (II.12)]

the contribution to $\Gamma_{B A}(\tau,|\underline{k}|)$ rrom sources near I'; this 18 obly moaningtul for an incoberent source

- complex correletion function [800 Eq. (II.34)? the wutual coborence botween bears $A$ and $B$ observed aftor beam B is Doppler shifted in Trequency [soe Eq. (V.4)]

- mutual coborence between light of different vavelengthe [nee Eq. (v.7a)] 
$B$

$\underline{6}$

$0^{2} 8$

$\Delta$

$\Delta k$

$\Delta$

$\theta$

$\theta\left(x^{\prime \prime}, y^{\prime \prime}, \underline{x}^{\prime}, \infty\right)$

$\lambda_{\mathrm{D}}$

s(x,t)

$s_{0}(\underline{r}, t)$

$s_{A}(\underline{r}, t), s_{B}(\underline{r}, t)$

$\tilde{s}_{g}(\boldsymbol{r}, t)$

p

$\sigma_{T}$

T focal spot size (In Sect. I.B)

a spall displacement of a source within a common source volume (in Sect. II.A.I) the small solld angle subtended at the source by an observed beam

the distance between adjacent beam-defining slits in a multiple-beam spectrometer the width of a spectrometer 1nstrument function a spectrometer baniw1dth, or the width of an observed spectral line, whlchever is narrower the angle at which two interfering beams Intersect at a screen

the alfference in phase between the transfer runctions $\phi_{A}$ and $\phi_{B}$ plasma Debye lergth scalar wave amplitude [from $\mathrm{aq}$. (I.3)] the amplitude of the l1ght accepted by a multiple-beam spectrometer amplitudes of the Ilght in beams $A$ and $B$, or of the $A$ and $B$ polarization components of the light

the light in beam 8 observed froc a moving frams of reference $[$ see Eq. $(\because .3 b)]$ cherge deneity

differential Thompon scattering cross section tibe inverse bandwidth of the spectrum analyzer used to obtain a signal spoctrum (In Sect.III.C) 
$\tau_{0}$

$\boldsymbol{T}_{\mathrm{A}}, \boldsymbol{T}_{\mathrm{B}}$

$\varnothing$

$\varnothing$

$\varnothing$

$\phi\left(\underline{r}^{\prime}, t\right)$

$\left.\begin{array}{l}\phi_{A}\left(\underline{r}^{\prime \prime}, \underline{r}^{\prime}, \omega\right), \\ \phi_{B}\left(\underline{r}^{\prime \prime}, \underline{r}^{\prime}, \omega\right)\end{array}\right\}$

$\phi_{j}(t)$

$\left.\phi_{1} \underline{x}^{(\ln )}\right)$

$\phi_{2}\left(\underline{r}^{(\text {out })}\right)$,

$\phi_{10}, f_{20}$

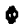

$\omega$

40

$\infty$

$w_{A}, \psi_{B}$
$=1 \nu_{0}$, a time interval characteristic of the $k_{\Delta}$ component of a light source distribution time required for beams $A$ and $B$ to travel through the calcite rhomb electromagnetic scalar tential angle subtended at a screen by one of a pair of interfering beams

$=\underline{k}_{B} \cdot \underline{r}_{B}-\underline{k}_{A} \cdot \underline{r}_{A}$, a phase factor in the measured mutual coherence

the phase of a nearly monochromatic source [see Eq. (II. 36)]

the two complex-valued transfer ifunctions of a mult1ple beam spectrometer the phase of nng of a set of point sources (in Chapter $v$ )

optical path lengths addad by the infut and output lenses of a multiple-beam spectramter constant contributions to $\phi_{1}$ and $\phi_{2}$ a wean path length for rays in a multipleboam spectrometer $[$ see Eq. (II. 30) ansular rrequency

a rharacteristic frequency of the $\underline{k}$ couponent o- a light source distribution

a frequency of a bearly monochromatic soure [seo Eq. (II. 36)]

the frequencies of light in beam $A$ and $B$ (In Chapter V) 
$\omega_{1}$

$\omega_{\mathbf{g}}$

$w_{B}$

$\omega_{\Delta}$ frequency of incident light

the frequency of one of a set of point sources (in Chapter $v$ )

frequency of scattered light

a difference frequency $\left(=\omega_{B}-\omega_{1}\right.$ or $\left.\omega_{B}-\omega_{A}\right)$ 
FOOTWOTES AND REFERENCES

\section{Chapter I}

1. Of particular importance here 1a progress made in the understanding and use of partially coberent light. This work is summarized in several recent books:

a. X. J. Beran and G. B. Parrent, Jr., Theory of Partial Coherence (Prentice-Hall, Englewood Cliffs, N. J., 1964).

b. E. L. O'Ne111, Introduction to Statistical optice (Add1son-Wesley, Reading, Nass., 1963).

c. L. Kandel and E. Wolf, Eda., Selected Papers on Coherence and Fluctuations in Light (Dover, New York, 1970). Several articlesin the series Progress in optic日, E. Wolf, Ed. (North-Holland Publishing Co., Amsterdem), slso pertain to this subject.

2. D1scussions of related quantum problems are avallable in several recent books on quantum optics:

a. R. J. Glauber, Ed., Quantum Optice, Proceedings of the Inte rnational school of Physics "Enrico Fermi" Course XIII, Bumber 1967 (Academic Press, Nev York, 1969).

b. S. M. Kay and A. Maitiand, Eds., Quantum Opt ICs, Proceedinge of the Tenth Scott1sh Sumwer School, 1969 (AcodemIc Prese, New York, 1970).

c. J. R. Kauder and E. C. O. Sudaroher, Pundamentale of Quantum Optics (Bonjamin, Now York, 1968). 
3. The general subject of plasma diagnostics 1s discussed in several books, including

a. R. H. Huddlestone and S. L. Leonard, Eds., Plasma Diagnost1c Technigues (Academic Press, New York, 1965).

b. H. R. Griem and R. H. Lovberg, Eds., Plame Physics, Vol. 9 of Methods in Experimental Physics, L. Marton, Ed.-In-Chlef (Academic Press, New York, 1970).

c. W. Lochte-Holtgreven, Ed., Plasma Diagnost1cg (NorthHolland Publishing Co., Amsterdam, 1968).

4. P. S. Rostler, W. S. Cooper, and W. B. Kunkel, MultipleBeam Spect roscopy, Bull. Am. Pays. Soc. 17, 986 (1972).

5. J. D. Jackson, Classical Electrodynamics (Wiley and Sons, New York, 1962), p. 180.

6. See, for example, S. L. Leonard, Basic Macroscopic Measurements, in Plasma Dlagnostic Techniques, R. H. Huddlestone and S. L. Leonard, Eds. (Acadewic Press, New York, 1965).

7. See References 3 and also

a. H. R. Griem, Plasma Spectroscopy (Mcrimw-H1ll Book Co., New York, 1964).

b. G. V. Marr, Plesma Spectroscopy (Elsevier, Amsterdam, 1968).

B. The mechenisms of bromsatrahlung and cyclotron emiosion are discussed in Chapter 15 of J. D. Jackson, Clessicel Electrodynamice (W1ley and Sone, Now York, 1962), and in Chapter 3 of P. C. Clcmow and J. P. Doughorty, Electrodynamics of Perticlos and Pleames (Addison-Wealoy, Reading, Ms8., 1969). 


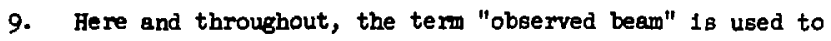
denote $a$ bundle of rays accepted by an optical system. This does not mean incident 11ght, the light 1 s all emitted by the plesme.

10. J. Richter, Radiation of Hot Gases, (Sect. 2.1) In Plasme Diagnost1cE, W. Lochte-Holtgreven, Ed. (North-Holland Publishing Co., Amsterdam, 1968).

11. H. R. Griem, Plasma Spectrobcopy (McG-aw-Hill Book Co., Hew York, 1964), Ch. 7 .

12. F. C. Jahoda and G. W. Sawyer, Optical Refractivity of Plasmas, in Plama Physics, H. R. Griem and R. H. Lovberg, Eds., Vol. 9 of kethods in Experimental Physics, L. Marton, Ed.-In-Chief 'Academic Press, New York, 1970).

13. A. H. A. Adler and D. R. White, Optical Interferometry, in Plasma Diagnostic Techniques, R. H. Buddlestone and S. L. Leonard, Eds. (Acaderic Press, low York, 1965). b. U. Ascol1-Bartol1, Plame Diagnostics Based on Refrectivity, in Phycics of Hot Plaames, B. J. Rye and J. C. Taylor, Eds. (Proceedings of the Ninth Scott1sh Sumer School, 1968) (Ol1ver and Boyd, Edsnburgh, 1970).

14. K. L. Borled, Observation of Vertical Incidence scatter from the Ionieghere at $41 \mathrm{Mc} / \mathrm{sec}$. Phys. Rev. Letters 1, 454 (1958).

15. 2. M. N. Rosenbluth and M. Rostaker, Scattering of Electromenetic theves by Alonequilibrlum Plasma, Phys. Fluids 2. $776(1962)$. 
b. J. P. Dougherty and D. T. Farley, A Theory of Incoherent Sc-ttering of Rado Weves by a Plasma, Proc. Royal Soc* (London) A259, 79 (1960); and D. T. Farley, J. P. Dougherty, and D. W. Barron, A Theory of Incoherent Scattering of Radio Waves by a Plesma II. Scattering In a Mnetic Field, Proc. Royal Soc. (I.ondon) A263, 238 (1961).

c. E. E. Salpeter, Electron Dens1ty Fluctuations in a Plasma, Phys. Rev. 120, 1528 (1960); and Effect of the Magnetic Field in Ionispheric Backscatter, J. Geophys. Res. 66, 982 (1961).

d. J. A. Fejer, Scattering of Radio Waves by an Ionized Gas In Thermal Equilibrtum, Can.J. Phys. 38, 1114 (1960).

16. The literature of this type of experiment is quite extensive. The general state of the subject ind the avallable reference material are summarized in several recent reviews:

a. D. E. Evans and J. Katzenste1n, Laser Light Scattering In Laborrtory Plarmas, Reports on Progress in Fhysics 32, 207 (1969).

b. S. A. Ramsden, Light Scattering Experiments In Physics of Hot Plasmes, B. J. Rye and J. L. Taylor, Eds., (Proceedings of the Ninth Scott16b Summer School, 1968) (Oliver and Boyd, Ed1nburgh, 1970).

c. A. W. Desilve and G. C. Goldenbeum, Plasma Dlagnstoics by Light Scattering, in Plason Phyalcs, H. I Griem and R. H. Jovberg, Eds., Vol. 9 of Methods In Experimental 
Physics, L. Marton, Ed.-In-Chief (Academlc Press, New York, 1970; .

17. That 1s, the coherence length of the incident light exceeds the dimensions of the veattering volume and the wilth of the laser spectrum is much less than that of features in the spectrum of the scattered light.

18. In the limit discussed here (the "Born approximation") the electric field of the scattered light is neglected in calculating the particle accelerations. The ladex of refraction is assumed equal to unity and the small effects of plasma particles are calculated separately. An alternate approach is to consider the scattering as due to variation In the index of refractiou. Sce, for exemple, H. G. Booker, A Theory of Radio Scettering in the Troposphere, Proc. IRE 38, 401 (1950).

19. See J. D. Jeckson, Clesgical Electrodvnamics (Wiley and Sons, New York, 1962), Sect. 14.1; or W. K. H. Panof sky and M. Phillips, Classicel Electricity and Kagnet1sm (AddisonWesley, Reading, Mase., 1955).

20. E. R. C. Devideon, Mpthods in Vonlinear Plesma Theory (Acadomic Press, New Ycrk, 1972).

b. V. N. Tsytovlch, Nonlinear Effecte in Plaeme (Plenum Press, Now York, 1970).

21. See F. Relf, Fundemontals of Statletical and Thermal Physics (Moorew-Hill Book Co., Mev York, .965), g. 585; or C. Kittel, Elementery Statiotical Paysios (Wiloy and Sons, Now York, 
1958), p. 133.

22. See, for example, S. E. Schwarz, Plasma Diagnos1s by Means of Optical Scattering, J. Appl. Phys. 36, 1836 (1965).

23. See, for example, 0. A. Anderson, Laser Light Scattering Measurement of Plasma Density, Temperature, and Correlation Spectrum (Ph.D. Thesis), Lawrence Livermore Laboratory Report UCRL-50699; or D. A. Re111y, Light Scattering by Ion Thermal Fluctuations in a Dense Plasma (Ph.D. Thesis), Lawrence Berkeley Iaboratory Keport UCRL-20815.

24. H. M. Smith, Frinc1ples of Holography (IH1ley end Sons, New York, 1969).

25. a. Dennis Gabor, Holography, 1948-1971, Sclence 177, 299 (July 1972).

b. G. W. Stroke, An Int oduction to Coherent Optics and Hojogrephy (Academic Press, New York, 1969).

c. R. J. Collier, C. B. Burkhardt, and C. W. Lin, Opt1cal Hologrephy (Academic Press, New York, 1971).

26. a. L. Rogers, Experimenta In Diffraction Microscopy, Proc. Roy. Boc. (EdInburgh) E3, 193 (1952).

27. A. L. Wertz and N. O. Yourz, Measel Transformations of Images, in Optical instruments and Techniques, $\mathrm{K} . \mathrm{J}$. Hisbel1, Ed. (Chapman and Hall, London, 1962), pp. 305-12. b. No11s 0. Young, Ptotography without Lenses or Kirrors, Bky and Molecope, Jan. 1963, p. 8.

28. L. Mrtz, Another Optical Prosnel Transforwer, (advert1sewont) in J. Opt. Soc. An. 54, iv (1964). 
29. a. Gary Cochran, New Method of Making Fresnel Transforms with Incoherent Light, J. Opt. Soc. Am. 52, 615 (1965).

b. A. W. Lohmenn, Wave front Reconstruction for Incoherent Objects, J. Opt. Soc. Aln. 55, ${ }^{-55}$ (1965).

c. J. T. Winthrop and C. K. Worthington, X-Ray Microscopy by Successive Fourler Transformation, Phys. Jetters 15, 124 (1965).

30. a. George W. Struke and Robert C. Restrick III, Holography with Spatially Noncohereni IfBht, Appl. „4ys. Letters 7, 229 (1965). This work is described also by G. W. Stroke In An Introduction to Coherent Optics and HoloGraphy (Academ1c Press, New York, 1969), Sect. VI 9.

b. Gary Cochran, New Method of Making Fresnel Transforms with Incoherent Light, J. Opt, Soc. Am. 56, 1513 (1966).

c. P. J. Peters, Incoherent Holograms with Mercury Light Source, Appl. Phys. Letters 8, 2C9 (1966).

d. H. R. Worthington, Jr., Production of Holograms with Incoherent Illumination, J. Opt. Soc. Am. 56, 1397 (1966).

31. a. Adam Kozme and Norman Messey, Bias Level Reduction of Incoherent Holograms, Appl. Opt. 8, 393 (1969).

b. 0. Bryngdahl and A. Lohmann, One Dimensional Holography with Spatially Incoherent Light, J. Opt. Soc. Am. 58, $625(1968)$.

\section{Chapter II}

32. For a slightly different statement of the same comparison, one can note that scattered light is described by a complex 
valued amplitude and that the total scattered wave is found by adding a set of complex numbers, one for each scattering center. In tine two-beam spectroscopic observation, the mutual coherence between the light in beams $A$ and $B$ is also a complex valued quantity, and, if the source is incoherent, this coherence Just consists of contributions from the different source points. It 1 s this superposition of complex valued contributions to the mutual coherence which corresponds to summing up the complex amplitudes of scattered wavelets in a scattering experiment.

33. B. D. Cullity, Elements of X-Ray Diffraction (Addison Wesley, Reading, Mass., 1956).

34. G. B. Renedek, J. B. Lastovka, K. Fritsch, and T. Greytak, Brillouin Scattering in Liquids and Solics Using Low Power Lasers, J. Opt. Soc. Am. 54, 1284 (1964).

35. See, f.cr example, L. J. Cutrona, E. N. Leith, L. J. Porcello, and W. E. VIvian, on the Application of Coherent Optical Processing Techniques to Synthetic-Aperture Radar, Proc. IEEE 54, 1026 (1066).

36. See Max Born and Emil Wolf, Principles of Cptics (Pergamon Press, Oxford, 1970), p. 275.

37. a. F. Durst, A. Melling, and J. H. Whitelew, Laser Anemometry: A Report on EUROMECH 36, J. Fluis Mech. 56, pt. $1,143(1972)$.

b. Hartmut H. Bos: ', W. J. Hiller, and G. E. A. Meier, Nolse-Cancelling SIgnal Difference Method for Optical 
Velocity Measurements, J. Phys., E (London) ㅁ, 893 (1972).

38. See Ref. 5, p. 186.

39. M. Francon and S. Mallick, Polarization Interferometers (Wiley and Sons, New York, 1971).

40. See Ref. 5, p. 188.

41. See H. B. Dwight, Tables of Integrals and Other Mathematical Tata (Macmillan, New York, 1961), p. 223 (integral 8:8.713).

42. E. Wolf, Light F'luctuations as a New Spectroscopic Tool, Japan T. Appl. Phys. 4, Suppl. I, I (1965).

43. Also, an additional factor of $2^{(m+n)}$ has been included in Eq. (II.34). This is to compensate for a difference of a factor of two in the definition of the analytic signal. See the discussion preceding Eq. (E.14) in Appendix E.

\section{Chopter III}

44. R. Henbury Brown and R. G. Twiss, Correlation Between Photons in Two Coherent Beams of Light, Nature 177, 27 (1956) and A Test of a New Type of Stellar Interferometer on Sirius, Nature 178, 1046 (1956), both reprinted in Selected Papers on Coherence and Fluctuations of Light, $I$. Mandel and E. Wolf, Eds. (Dover, New York, 1970), pp. ¿'j0-25r.

45. R. Hanbury Brown and R. Q. Twiss, Interferometry of the Intensity FIuctuations in Light, I. Basic Theory: The Correlation Between Photons in Coherent Beams of Radiation, Proc. Roy. Soc. (London) A.242, 300 (1957), reprinted in Selected Papers on Coherence and Fiuctuations of L1ght, 
I. Mandel and E. Wolf, Eds. (Dover, New York, 1970), pp. 272324.

46. E. M. Purce11, The Question of Correlation Between Photons in Coherent Light Rays, Nature 178, 1449 (1956), reprinted in Selected Papers on Coherence and Fluctuations of Light,

L. Mandel and E. Wolf, Eds. (Dover, New York, 1970), pp. $270-271$.

47. In observetions of high-frequency phenomena, however, some variation in a could be tolerated. If the signal frequency were greater than the frequency of the variations in the Ine integrals of $n$, the effect of interference could be seen but the apparent frequency of the phenomenon would be affected by the time dependence of the optica.. path lensths.

48. R. J. Glauber, Photon Counting and Field Correlations, in Physics of Quantum Fitctronics (conference proceedings), P. I. Kelley, B. Leik, and P. E. Tannenwald, Eds. (McGrawHill Book Co., New York, 1966), pp. 788-811.

49. L. Mandel, Fluctuations of Light Beains, in Progress in Optics, E. Wolf, Ed. (No:th-Holland Publishing Company, Amsterdam, (1963), vol. II, pp. 181-248.

50. F. Robben, Noise in the Meesurement of Light witl Photomult1pliers, Appl. Opt1es 10, 776 (1971).

\section{Chepter IV}

51. 11. A. Hess, Study of a Beam-Plasma Instability by Spectroscoplc Methods (Ph.D. Thes1s), Iawrence Berkeley Laboratory Report LBI-1531, December 1972. 


\section{Chapter V}

52. H. 2. Curmins and H. I. Swinney, Light Beating Spectroscopy in Progress in Opties, E. Wolf, Ed. (Nurth-Holland PublishIns CJ., Amsterdam, 1970), Vol. VIII, pp. 133-200.

53. To shift the frequency light one can use a Bragg cell in which light is reflected from a sound wave in a liquid. See H. Z. Cumbins and N. Knab7e, Single S1debars: alulation of Coherert Light by Bregg Reflection from Acoustical Weves, Proc. IFEE 51, 1246 (1963). There are also electro-optic methods for frequency shifting of light waves. See C. F. Is.hrer, V. J. Fowler, and L. R. Bloom, Single Sidebenä Suppressed-Carrier Modulation of Coherent Light F.sams, Proc. IRE 50, 1827 (1962); and C. F. Buhrer, D. Baird, and E. M. Conwell, Optical Frequency Shifting by Electro-optic Effect. Appl. Phys. Letters 1, 46 (1962).

54. See M. A. Pugay and J. W. Hansen, An Ultrafast Light Gate, Appl. Phys. Letters 15, 192 (1969).

55. a. Arthur Ruark, Fast Modulation Effects in the Optical Region, Phys. Rev. 73, 181 (1948).

b. A. Theodore Forrester, Richard A. Gudmundsen, and Philip 0. Jobnson, Photoelectric Mixing of Incoherent Light, Phys. Rev. 99, 1691 (1955), reprinted in Selected Papers on Coherence and Fluctuations of Light, L. Mandel and E. Wolf, Eds. (Dover, New York, 1970), pp. 220-229. 


\section{Appendix C}

56. This is equivalent to a requirement that the first slit be smaller than the maximum of a single slit diffraction pattern of the second slit. That is, the path length from any point across the width of one to any point across the vioth of the other must vary by less than half a wavel ingth.

57. Indeed, it is this which defines the beams: If the source is outside $\mathrm{a}$ beam, then the resulting distribution of phase across the first slit will be such as to destructively interfere at the second slit; the light which passes through the first slit is going in another direction. The first slit is many wavelengths wide. The light retains "memory" of direction to an accuracy slightly less than needed to cistinguish the small angle subrended by the second slit. This is, of course, fust the connection between geometrical optics ("beams A and $B "$ ) and the physical optics which determines the forms of the interference fringes and the associated polarizatio: patterns.

58. In using such s system, the optics would heve to be rather carefully aligned, so that all of the light transwitted by the collimator would then ente: the spectrometer. One right even want to remove the spactrometer entrance slit, and Just. let the second lens focus the collimated light into an image of a slit at the samc place.

59. One solution is to uee a series of two rhombs, the second rotated $90^{\circ}$ w.th respect to the first about the axis of the 
system. Then each polarization is extraordinary in one rhomb or the other and the differences in optical path length introduced will cancel, but the lateral displacements, which are perpendicular, will not. Thls combination of elements is called a Savart plate, or "polariscope". (See Ref. 39.)

\section{Appendix E}

60. A. A. Kharkevich, Spectra and Analys1s (Consultants Bureau, N. Y., 1966).

61. a. R. M. Fano, Short-Time Autocorrelation Functions and Power Spectra, J. Acoust. Soc. Am. 22, 546 (1950).

b. C. H. Page, Instaneous Power Spectra, J, Appl. Phys. 23, 103 (1952).

c. D. G. Lampard, Generalization of the Wiener-Khintchine Theorem to Nonstationary Processes, J. Appl. Phys. 25, 802 (1954). 